\title{
Evaluation of Bank Branch Growth Potential Using Data Envelopment Analysis
}

By:

\section{Alex E. LaPlante}

Center for Management of Technology and Entrepreneurship

Faculty of Applied Science and Engineering

University of Toronto

200 College St.

Toronto ON, M5S 3E5, Canada

A thesis submitted in conformity with the requirements

for the degree of Masters of Applied Science

Department of Chemical Engineering and Applied Chemistry

University of Toronto

(C) Copyright by Alex E. LaPlante, 2012 


\title{
Abstract
}

\section{Evaluation of Bank Branch Growth Potential Using Data Envelopment Analysis}

\author{
Alex LaPlante \\ Masters of Applied Science \\ Graduate Department of Chemical Engineering \\ University of Toronto
}

2012

Banks occasionally employ frontier efficiency analyses to objectively identify best practices within their organizations. Amongst the frontier efficiency analyses identified in the literature, Data Envelopment Analysis (DEA) was found to be one of the leading approaches. DEA has been successfully applied in many bank branch performance evaluations using traditional intermediation, profitability and production approaches. However, there has been little focus on assessing the growth potential of individual branches.

This research presents six models that examine four perspectives of branch growth. Each model was applied to the branch network of one of Canada's top five banks to gauge the growth potential of individual branches and to provide tailored improvement recommendations. Using various analysis methodologies, the results of each model were examined and their functionality assessed. Based on these findings, three models were deemed to produce significant results, while the remaining three failed to attain viable results. 


\section{Acknowledgements}

The journey towards the completion of this thesis started far before I ever set foot on the University of Toronto Campus. Throughout my life I have been blessed with an abundance of people who have motivated me to pursue my dreams and who have provided the guidance and support necessary to attain them. Although the list is seemingly endless, I am forever grateful to each one of them.

Additionally, there are several people who deserve acknowledgement for their more direct role in the completion of my thesis. First and foremost I offer my sincerest gratitude to my supervisor, Dr J.C. Paradi, who has supported me throughout my thesis with his patience, knowledge and sincerity. His sound advice and benevolent mentorship were great assets; helping to guide many important decisions in both my academic and everyday life. Dr. Paradi's devotion to his students and passion for teaching was an incredible inspiration and his candid nature was very much appreciated. One could not wish for a better or more supportive supervisor. I would also like to thank the Canadian Bank with which I worked for their gracious cooperation in providing the necessary data and for their aid in understanding their unique bank metrics. I would especially like to thank Daymond L., Catherine Y. and Jose R. for their enthusiastic involvement and insight.

The Center of Management of Technology and Entrepreneurship (CMTE) has provided a wonderful academic environment to learn and grow. It has been an honour to share my graduate experience with such an intelligent and talented group of individuals. In particular, I would like to thank Sau Yan Lee for sharing his eclectic repertoire of information and for providing his honest culinary critiques on my baked goods. I have very much enjoyed the plethora of deviant and entertaining conversations we have shared. This unlikely friendship has truly made my stay at CMTE that much better.

Beyond my CMTE colleagues, I must acknowledge my "Toronto family" who have been a great source of inspiration and support during my time spent away from home. We have shared many amazing and unforgettable memories which I will cherish for life. Andreea Pirvulescu has provided an unwavering friendship which has grown into an incredible sisterhood. Dillon Fuerth has been a constant source of stability and loyalty unlike any I have experienced before. Oren Kraus has provided a reason to bake continuously, as well as shown me amazing kindness and warmth. 
Finally, I thank my wonderful parents for always believing in me and for encouraging me to follow my dreams. I would be nowhere near the person I am today without their guidance and support. I am forever grateful for their sacrifices, the opportunities they have afforded me and the devotion they have shown to parenthood. Words cannot express how much they mean to me and how blessed I feel to be their daughter. 


\section{Table of Contents}

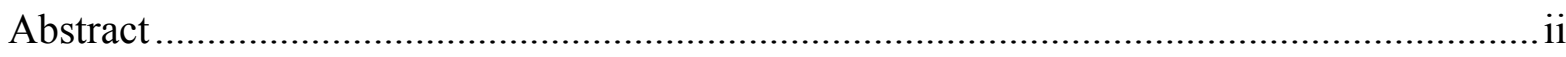

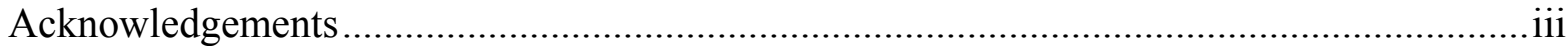

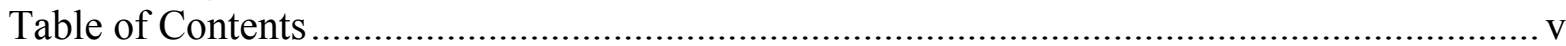

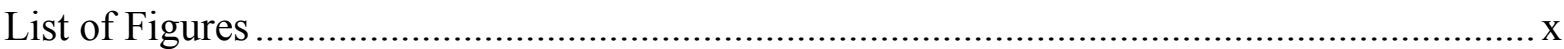

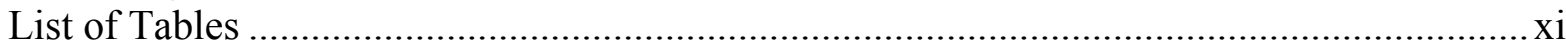

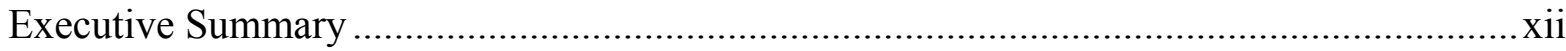

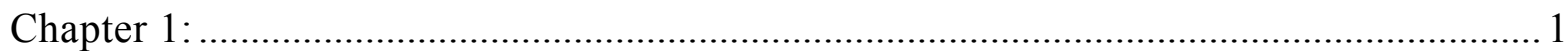

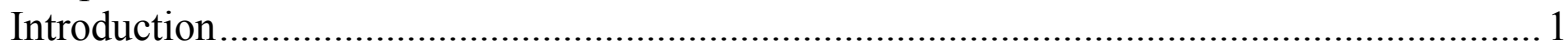

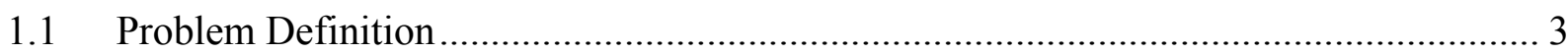

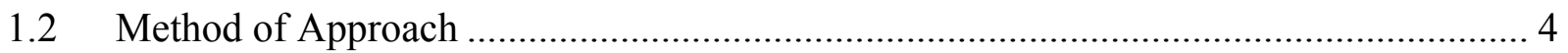

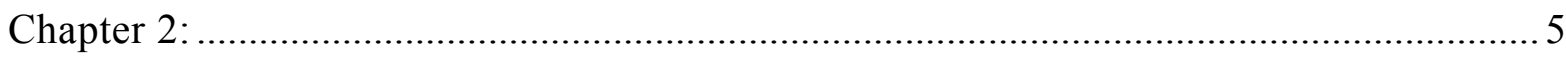

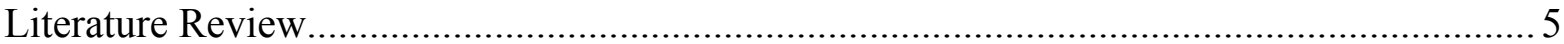

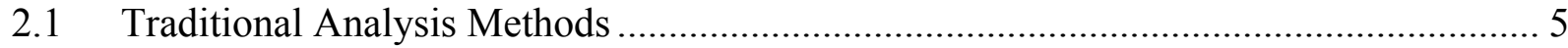

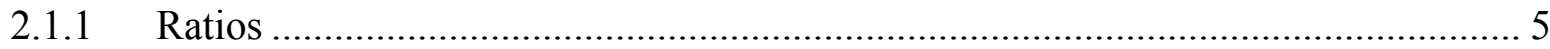

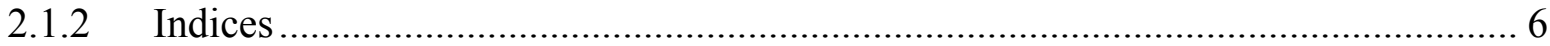

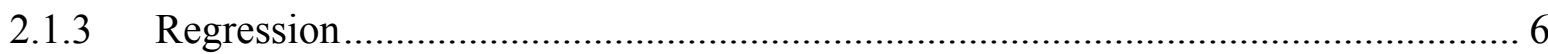

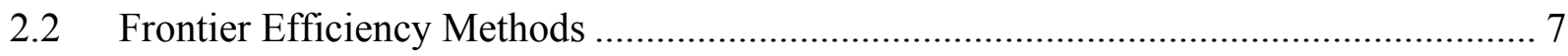

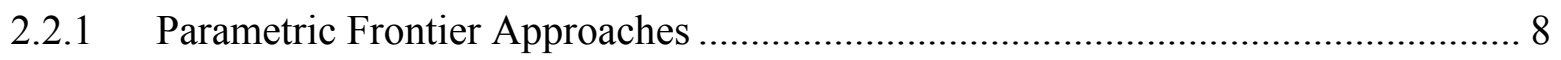

2.2.1.1 Stochastic Frontier Analysis (SFA) …………............................................. 9

2.2.1.2 Distribution- Free Approach (DFA) ……………………………………..... 9

2.2.1.3 Thick Frontier Approach (TFA) ………………....................................... 10

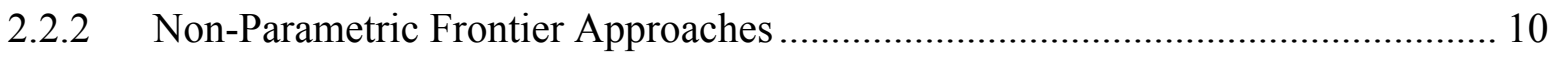

2.2.2.1 Data Envelopment Analysis (DEA) ……………..................................... 10

2.2.2.2 Free Disposal Hull (FDH) ................................................................ 11

2.3 DEA in Bank Branch Performance Evaluation........................................................... 11

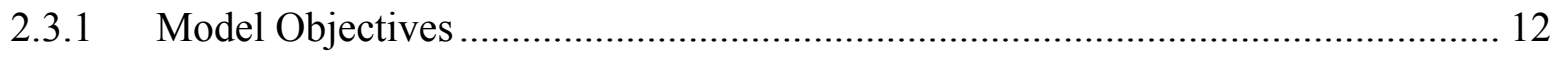

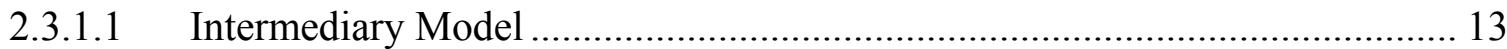

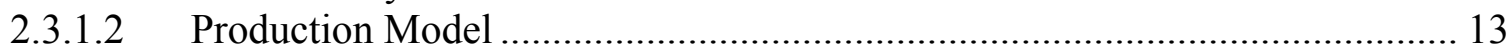

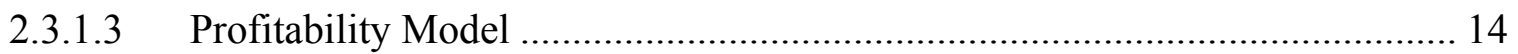

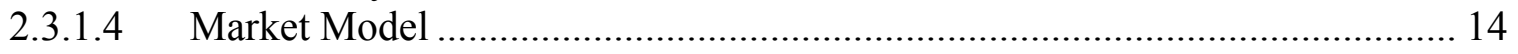

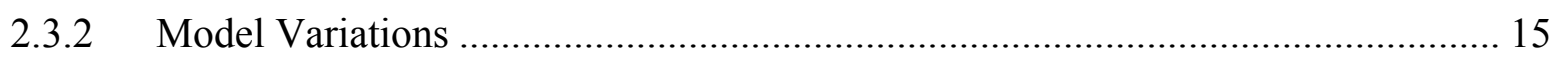

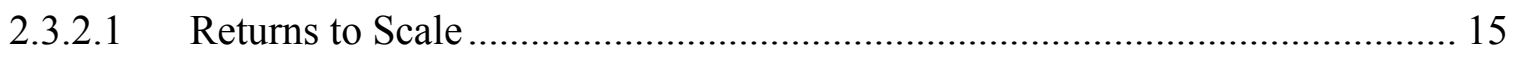

2.3.2.2 Input Oriented vs. Output Oriented ……………...................................... 15

2.3.2.3 Multi-Stage DEA Analysis .................................................................... 16

2.3.2.4 Relevant Extensions .............................................................................. 16

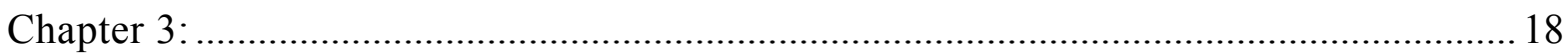

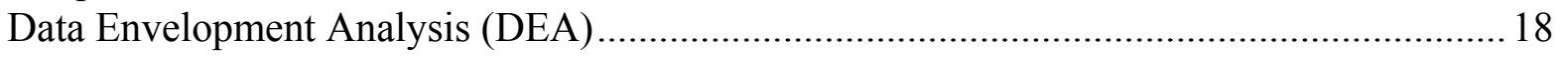




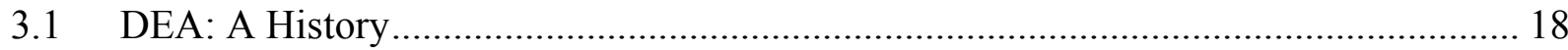

3.2 DEA Theory and Mathematical Formulation............................................................. 19

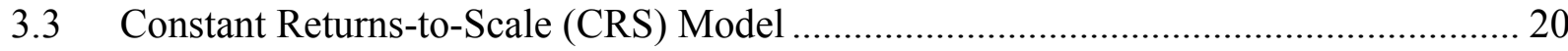

3.3.1 Input Oriented CRS Model ………………………....................................... 22

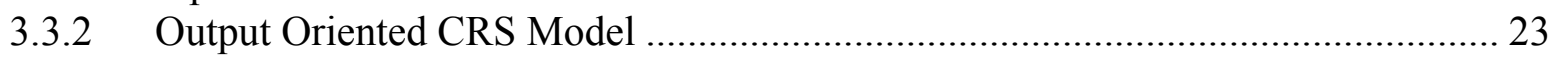

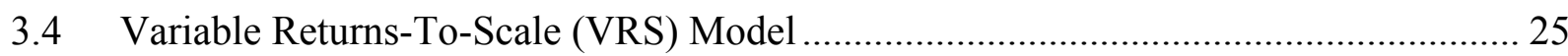

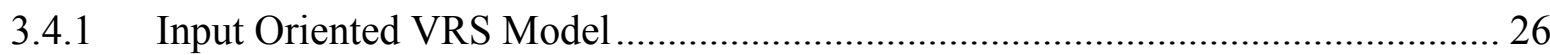

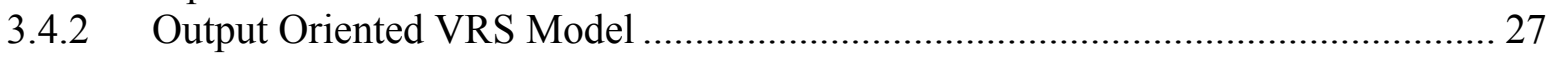

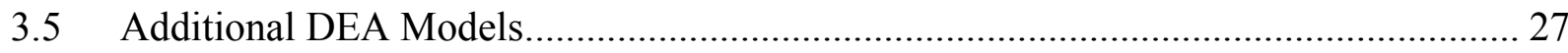

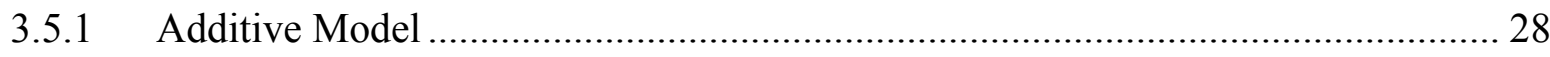

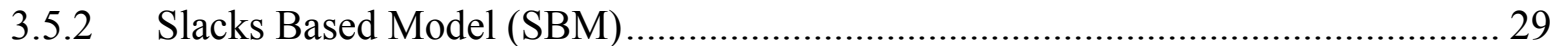

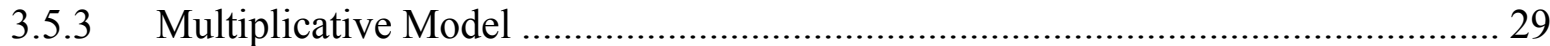

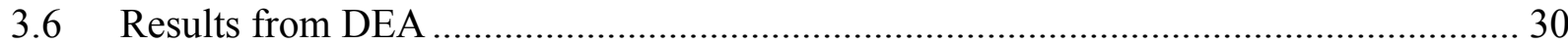

3.6.1 Technical and Scale Efficiency ......................................................................... 30

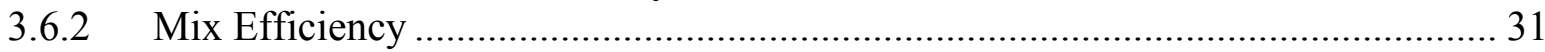

3.6.3 Peer Groups and Target Setting ......................................................................... 31

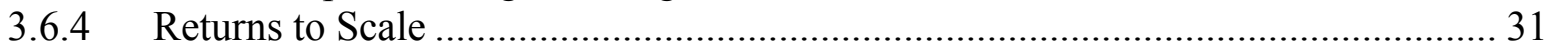

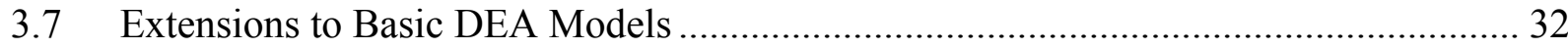

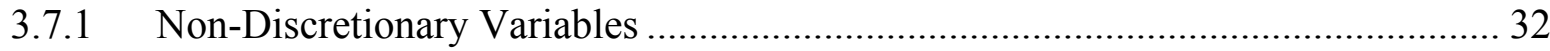

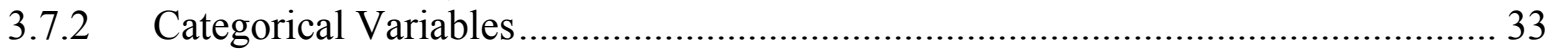

3.7.3 Restricted Multiplier Models ………………....................................................... 33

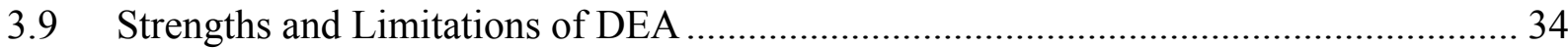

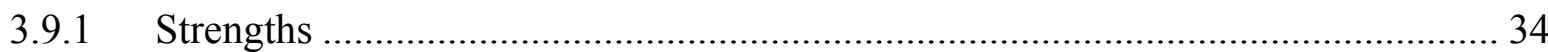

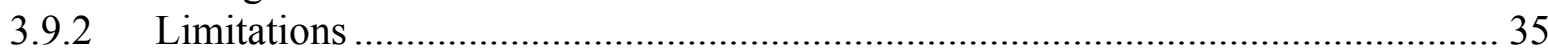

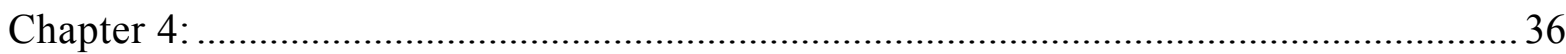

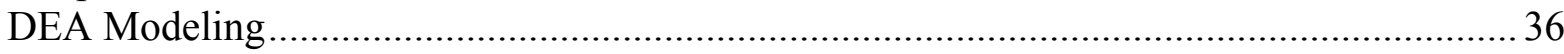

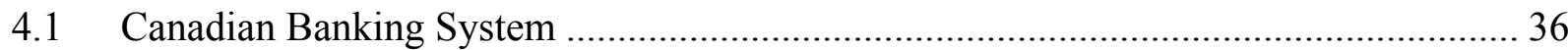

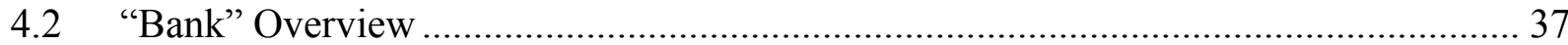

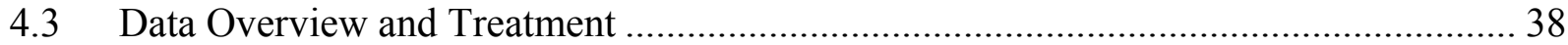

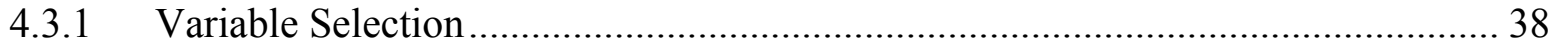

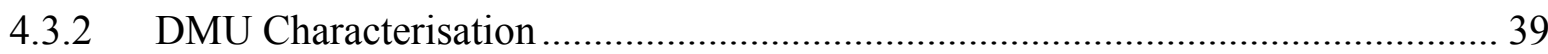

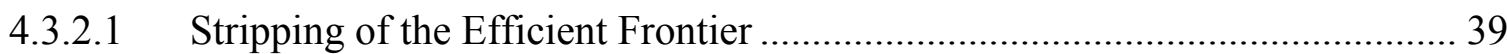

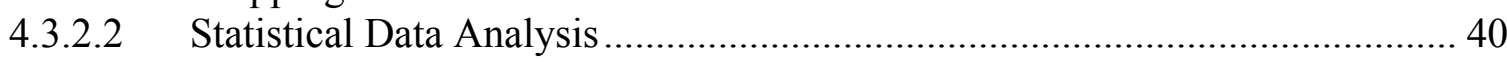

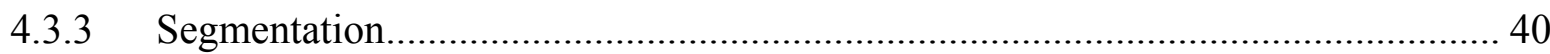

4.3.4 Local Analysis ......................................................................................... 41

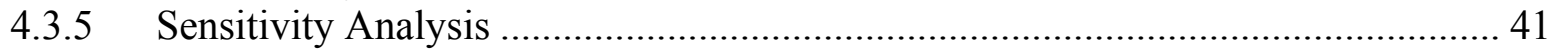

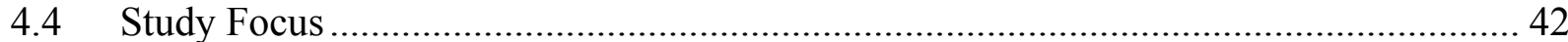

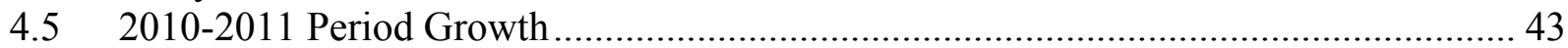

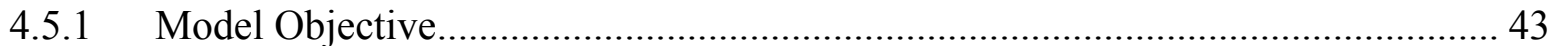

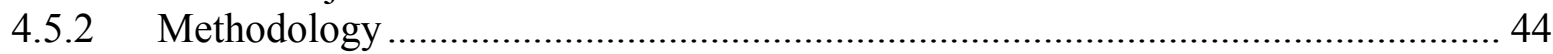

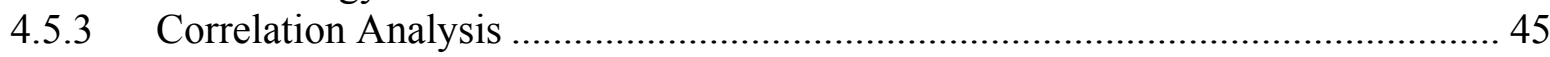

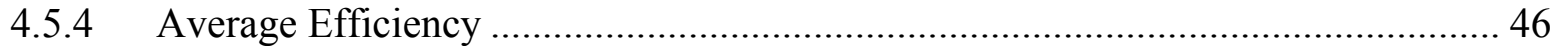

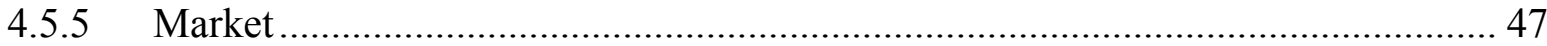

Multi-Stage Evaluation of Bank Branch Consolidation and Growth Potential Using DEA vi 


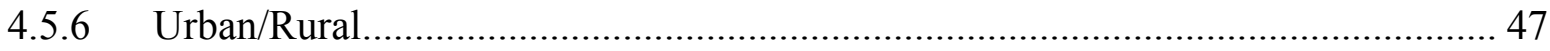

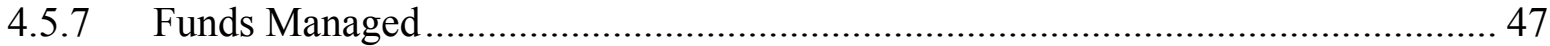

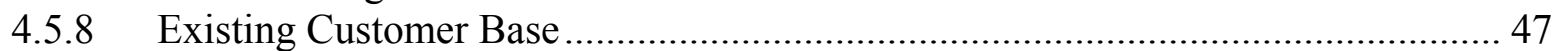

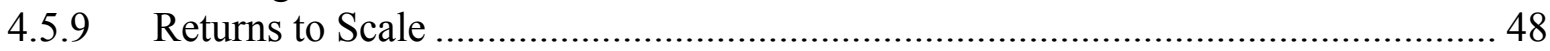

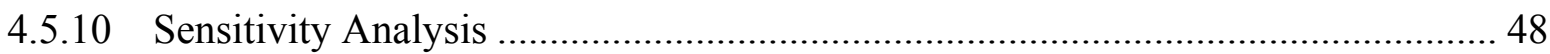

4.6 Lost Accounts/Gained Accounts Model ………………………............................ 50

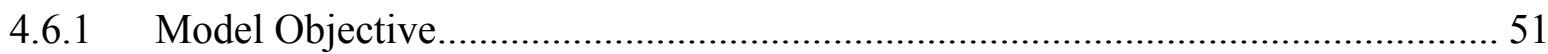

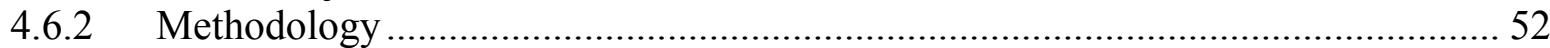

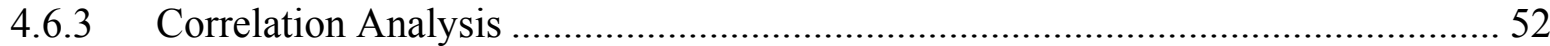

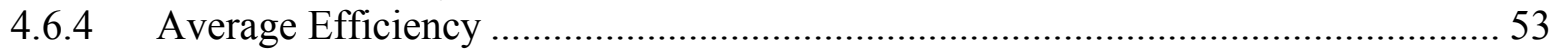

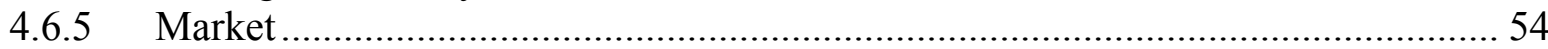

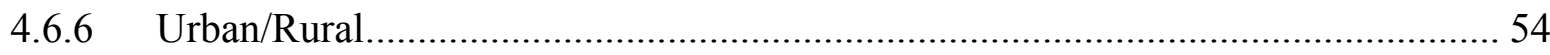

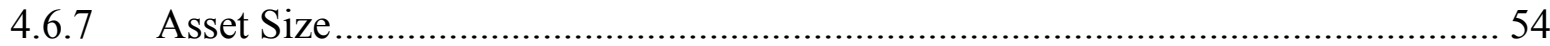

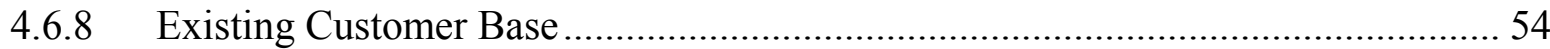

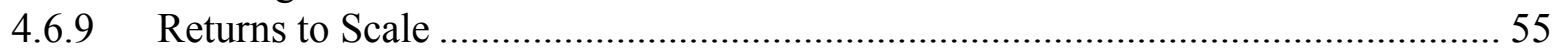

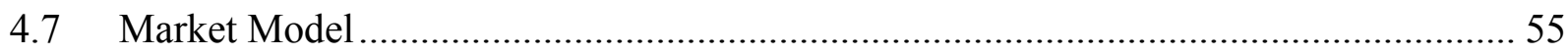

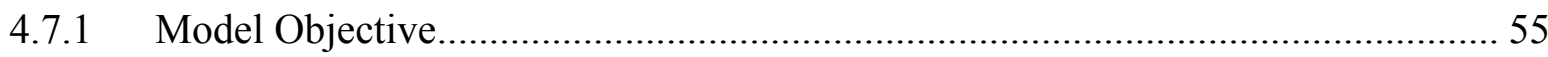

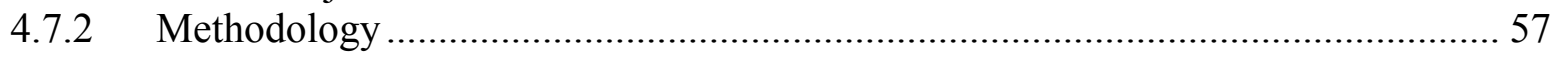

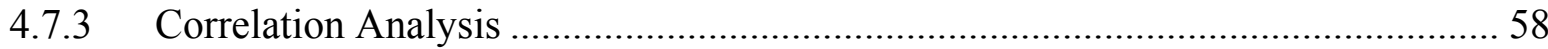

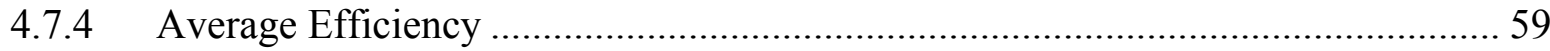

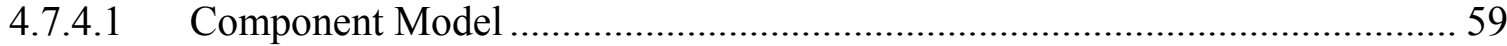

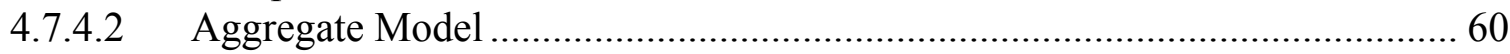

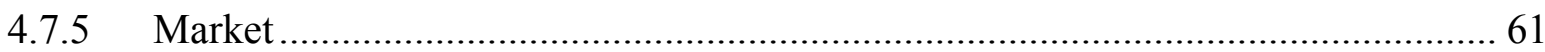

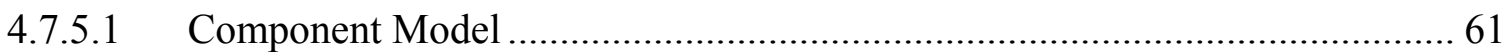

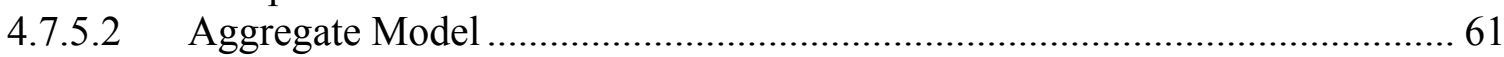

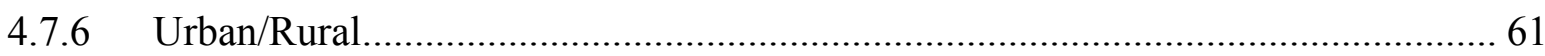

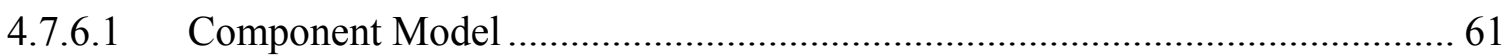

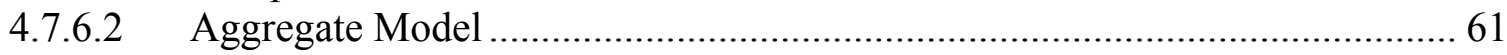

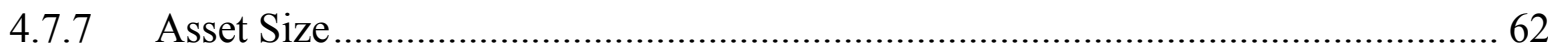

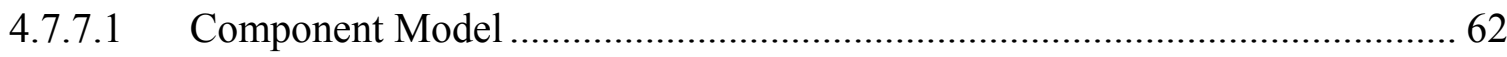

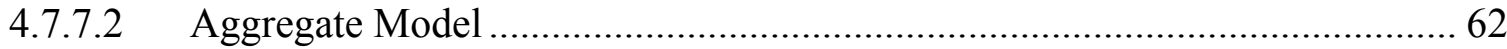

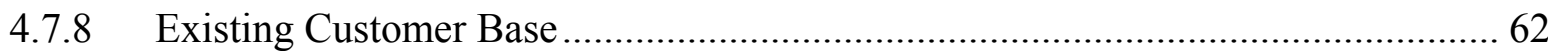

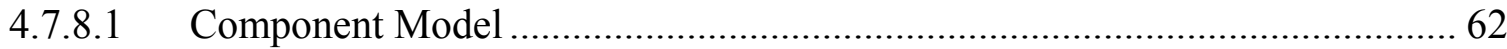

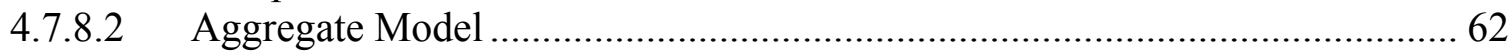

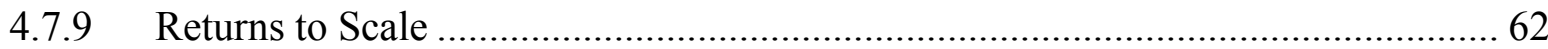

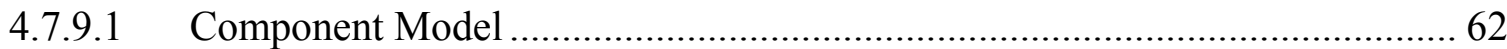

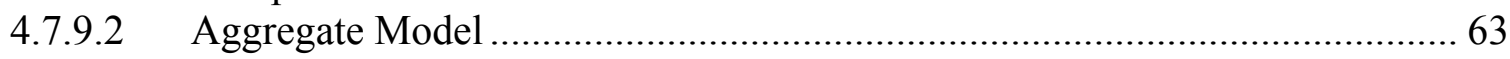

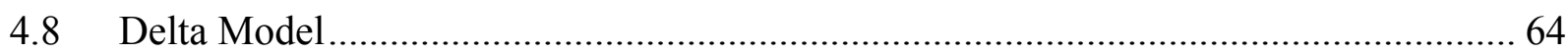

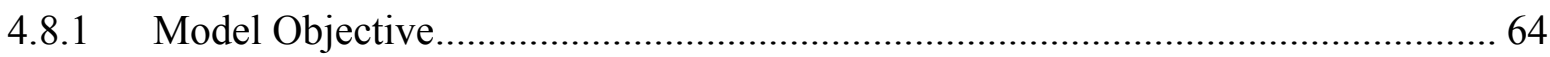

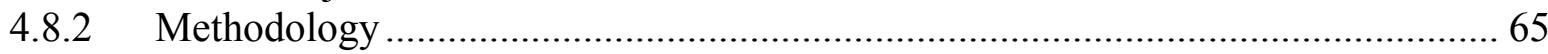

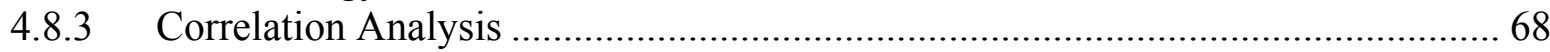

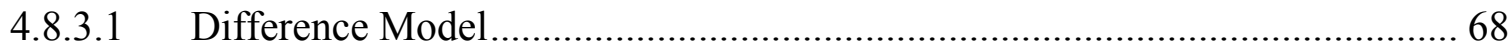

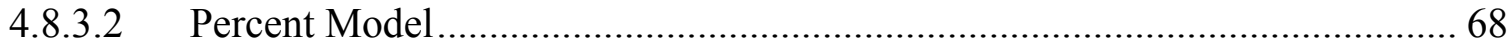

Multi-Stage Evaluation of Bank Branch Consolidation and Growth Potential Using DEA vii 


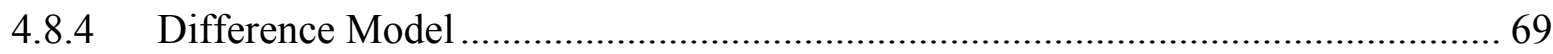

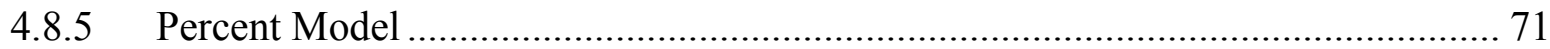

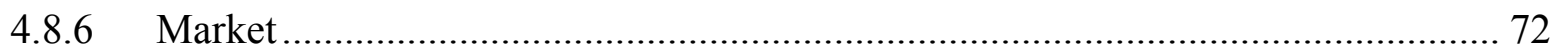

4.8.6.1 Difference Model: Stripping Efficient Units ............................................... 72

4.8.6.2 Difference Model: Data Analysis and Local DEA ....................................... 72

4.8.6.3 Percent Model: Stripping Efficient Units .................................................. 72

4.8.6.4 Percent Model: Data Analysis and Local DEA …........................................ 73

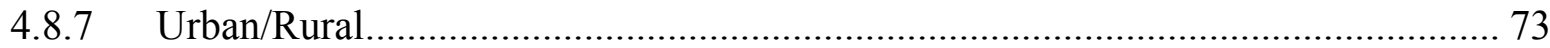

4.8.7.1 Difference Model: Stripping Efficient Units ................................................ 73

4.8.7.2 Difference Model: Data Analysis and Local DEA ...................................... 73

4.8.7.3 Percent Model: Stripping Efficient Units ................................................... 73

4.8.7.4 Percent Model: Data Analysis and Local DEA ......................................... 73

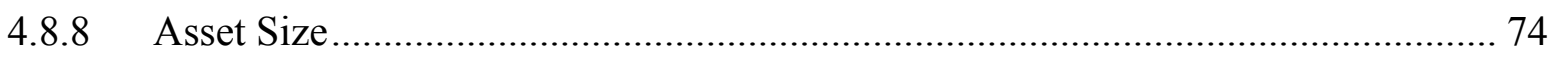

4.8.8.1 Difference Model: Stripping Efficient Units .............................................. 74

4.8.8.2 Difference Model: Data Analysis and Local DEA .................................... 74

4.8.8.3 Percent Model: Stripping Efficient Units ................................................. 74

4.8.8.4 Percent Model: Data Analysis and Local DEA ............................................. 74

4.8.9 Existing Customer Base ................................................................................ 75

4.8.9.1 Difference Model: Stripping Efficient Units ................................................ 75

4.8.9.2 Difference Model: Data Analysis and Local DEA ...................................... 75

4.8.9.3 Percent Model: Stripping Efficient Units ................................................... 75

4.8.9.4 Percent Model: Data Analysis and Local DEA …...................................... 75

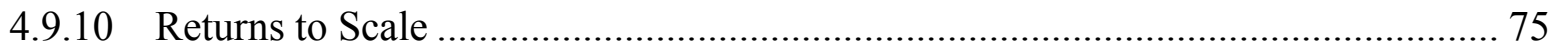

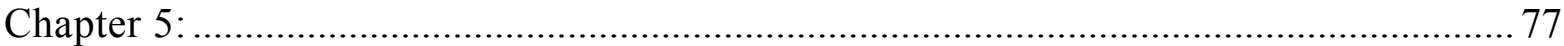

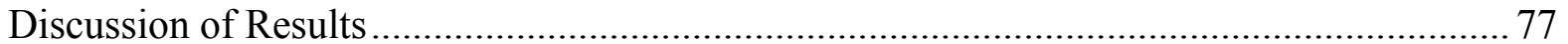

5.1 2010-2011 Period Growth Model …........................................................................... 77

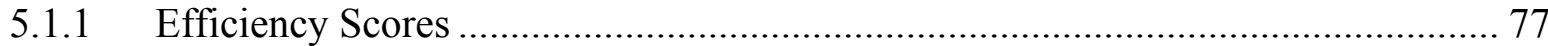

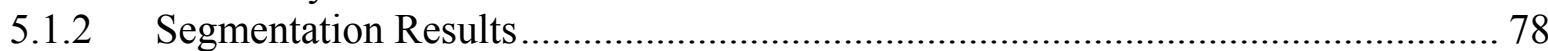

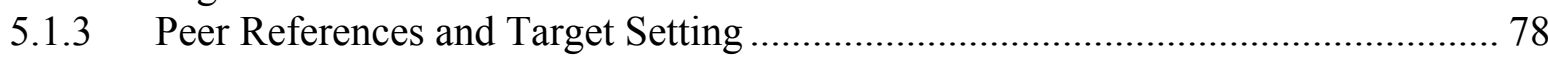

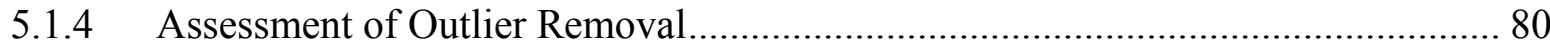

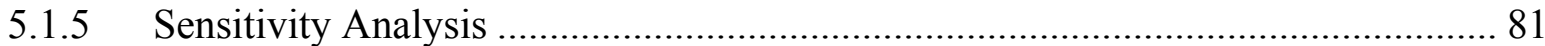

5.1.6 Overall Assessment of the Model .................................................................. 82

5.2 Lost Accounts/Gained Accounts Model ............................................................... 82

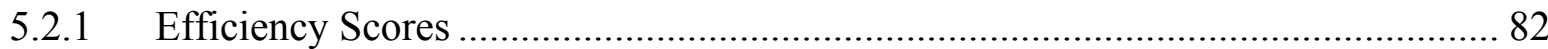

5.2.2 Segmentation Results ............................................................................ 82

5.2.3 Peer References and Target Setting .......................................................... 83

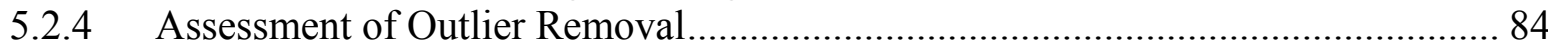

5.2.5 Overall Assessment of Model ............................................................................. 85

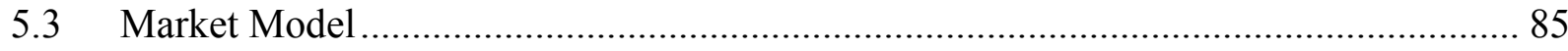

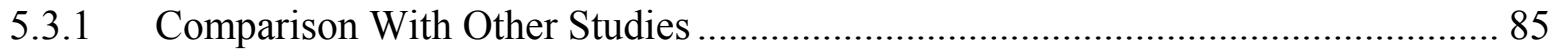

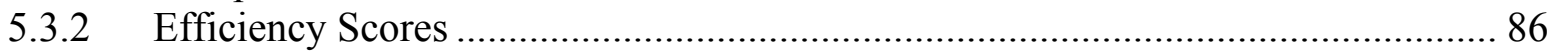

5.3.3 Segmentation Results ............................................................................... 86

5.3.4 Returns to Scale ........................................................................................ 87

5.3.5 Peer References and Target Setting ..................................................... 87

Multi-Stage Evaluation of Bank Branch Consolidation and Growth Potential Using DEA viii 


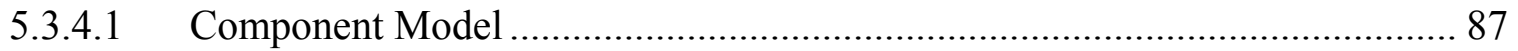

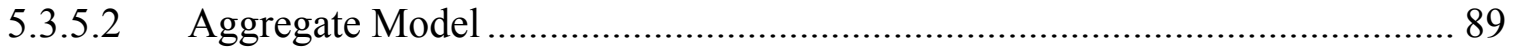

5.3.6 Assessment of Applied Methodology .............................................................. 91

5.3.7 Overall Assessment of Model ............................................................................ 91

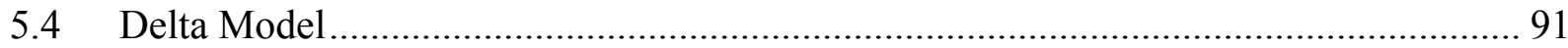

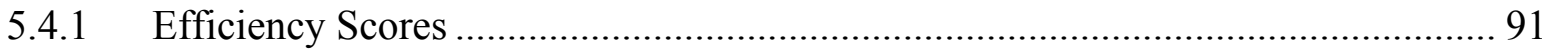

5.4.2 Segmentation Results ............................................................................. 92

5.4.3 Peer References and Target Setting .......................................................... 92

5.4.4 Assessment of Applied Methodology .............................................................. 93

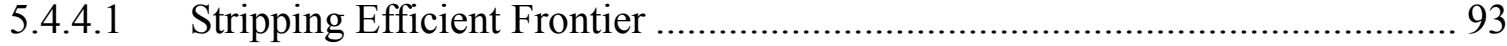

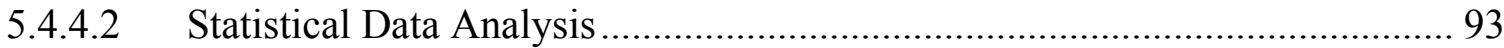

5.4.4.3 Local DEA .................................................................................... 93

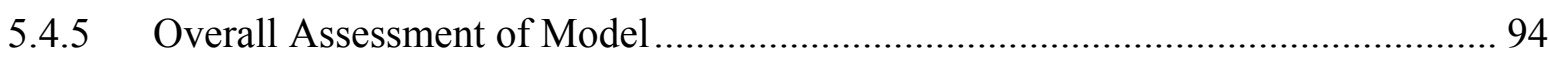

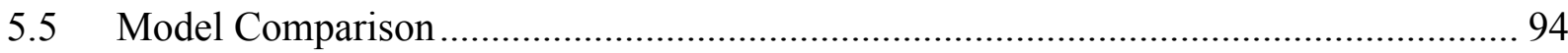

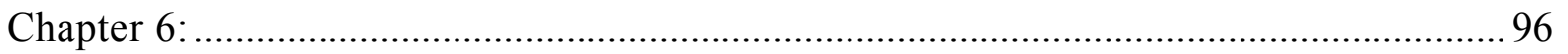

Conclusions and Recommendations ........................................................................ 96

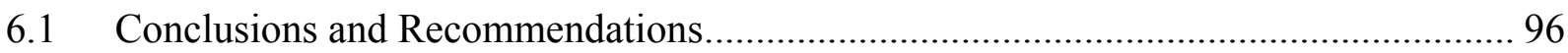

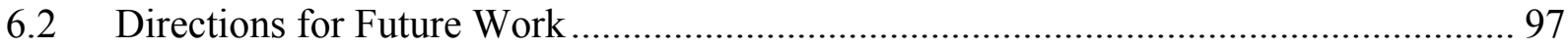

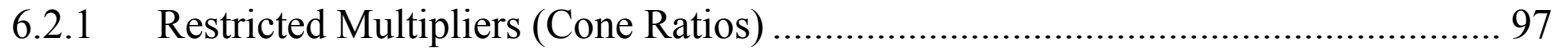

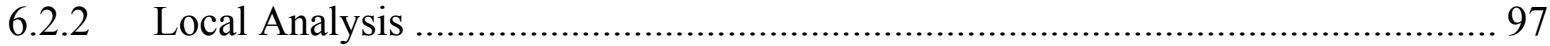

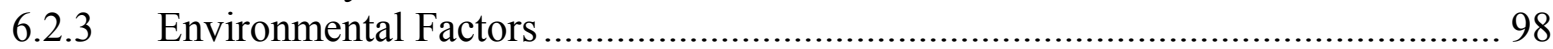

6.2.4 Extending the 2010-2011 Period Growth Model.................................................. 98

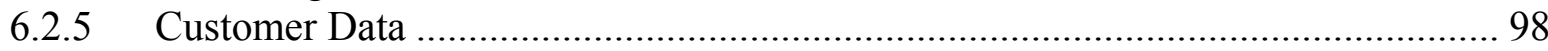

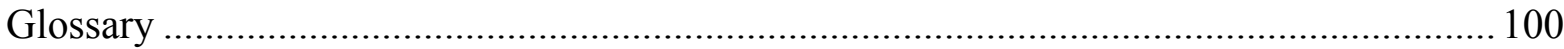

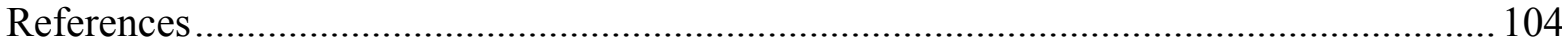

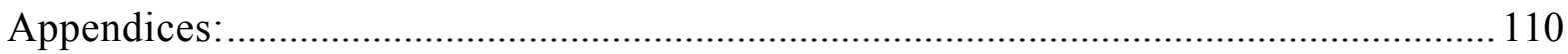

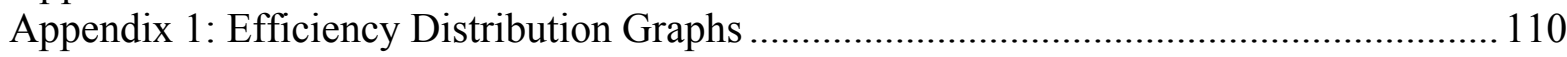

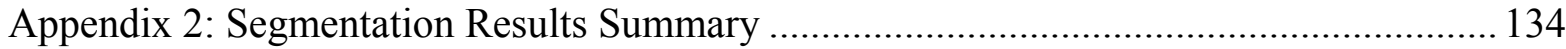

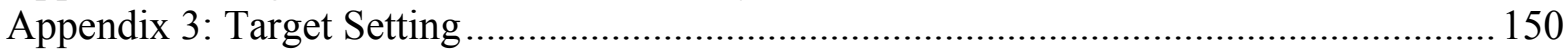

Multi-Stage Evaluation of Bank Branch Consolidation and Growth Potential Using DEA ix 


\section{List of Figures}

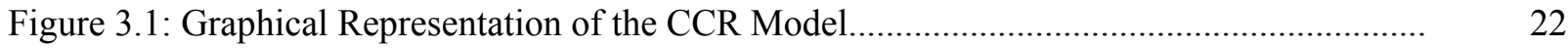

Figure 3.2: Graphical Representation of BCC Model.......................................................... 25

Figure 4.1: 2010-2011 Period Growth Model Execution Methodology.......................................

Figure 4.2: 2010-2011 Period Growth Model- Correlation Analysis............................................ 45

Figure 4.3: Lost Accounts/Gained Accounts Model- Correlation Analysis............................... 53

Figure 4.4: Market Model Methodology ...................................................................... 58

Figure 4.5: Market Model Correlation Analysis.................................................................. 59

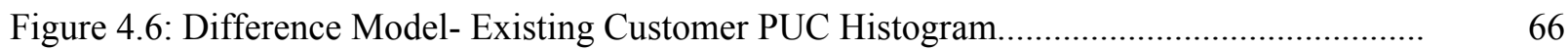

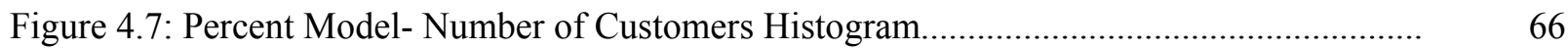

Figure 4.8: Delta Model 'Data Analysis and Local DEA' Execution Methodology..................... 67

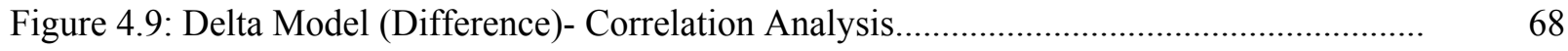

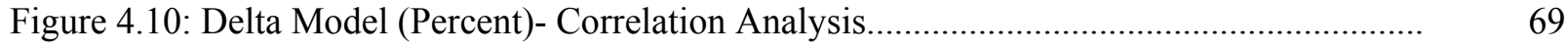

Figure 5.1: 2010-2011 Period Growth CCR Model Target Setting............................................ 79

Figure 5.2: 2010-2011 Period Growth BCC Model Target Setting........................................... 80

Figure 5.3: Lost Accounts/Gained Accounts CCR Model Target Setting................................... 83

Figure 5.4: Lost Accounts/Gained Accounts BCC Model Target Setting................................... 84

Figure 5.5: Two-Dimensional Representation of CCR and BCC Frontiers............................... 87

Figure 5.6: CCR Component Model Target setting........................................................... 88

Figure 5.7: BCC Component Model Target Setting.............................................................. 89

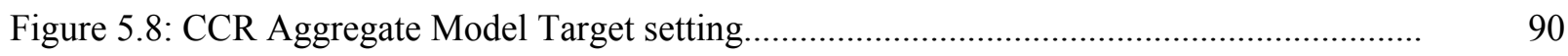

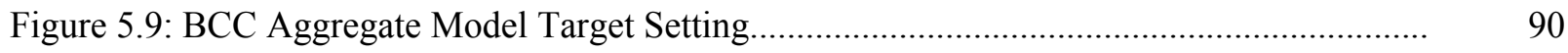




\section{List of Tables}

Table 4.1: Bank's Retail and Commercial Products and Services.............................................. 37

Table 4.2: Funds Managed Segments................................................................................ 40

Table 4.3: Existing Customer Base Segments................................................................... 41

Table 4.4: 2010-2011 Period Growth Model................................................................................. 43

Table 4.5: Summary of 2010-2011 Period Growth Model Average Efficiency Scores................ 46

Table 4.6: Efficiency Contribution Measure- CCR Model...................................................... 49

Table 4.7: Efficiency Contribution Measure- BCC Model......................................................... 49

Table 4.8: Wilcoxon Rank-Sum Test Results- CCR ........................................................... 50

Table 4.9: Wilcoxon Rank-Sum Test Results- BCC................................................................. 50

Table 4.10: Lost Accounts/Gained Account Model................................................................... 51

Table 4.11: Summary of Lost Accounts/Gained Accounts Model Average Efficiency Scores.... 54

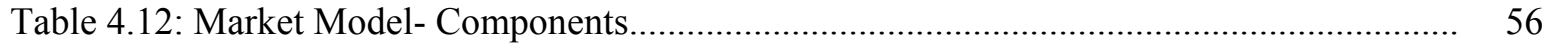

Table 4.13: Market Model- Aggregate........................................................................... 57

Table 4.14: Summary of Component Model Average Efficiency Results................................. $\quad 60$

Table 4.15: Summary of Aggregate Model Average Efficiency Results................................. $\quad 60$

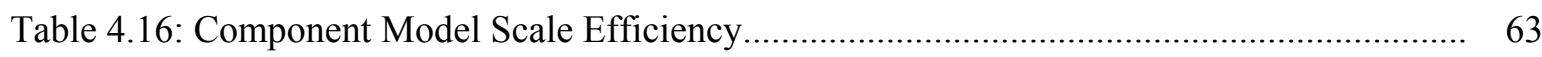

Table 4.17: Aggregate Model Scale Efficiency .................................................................. 63

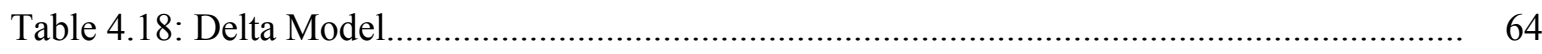

Table 4.19: Summary of Difference Model Efficiency Results- Stripping................................ 70

Table 4.20: Summary of Difference Model Efficiency Results- Data Analysis and Local DEA.. 70

Table 4.21: Summary of Percent Model Efficiency Results- Stripping.................................... 71

Table 4.22: Summary of Percent Model Efficiency Results- Data Analysis and Local DEA....... 72

Table 4.23: Summary of the Mix Efficiency Score for the Delta Models................................... 76 


\section{Executive Summary}

The main objective of this study is to evaluate the branch growth potential of a major Canadian Bank using a series of novel Data Envelopment Analysis (DEA) models. The approach used investigates growth efficiency from four different perspectives using six unique models. The results of each model are analyzed using various segmentations and their functionality assessed.

Along with a comprehensive review of the DEA literature and methodologies, a synopsis of the Canadian banking industry is presented and a general overview of the bank being studied is provided. Details pertaining to the more than 1000 branches evaluated, the regional and size segmentations used and the unique methodologies applied to each model are also discussed.

Unlike the more traditional production, profitability and intermediation approaches this study focuses on examining how well a branch grows its customer base and increases its sales of financial products. The use of multiple models allows for the investigation of many facets of branch growth. The six models and their objectives are discussed in detail and their justification for use is provided. Ultimately, three models were determined to provide significant results while the remaining three were not as effective.

The 2010-2011 Period Growth model uses 2010 product, fund and customer information as input variables and identical 2011 variables for the output. This symmetrical model offers the ability to assess the growth in products, funds and customers over the 2010 to 2011 yearend period and uses a set of unique models not yet seen in literature. Both CCR and BCC models were employed resulting in final average DEA scores of 0.89 and 0.94 , respectively. The model provides good discriminatory power and successfully identifies the best performers. It follows that this model has been deemed to be successful in achieving its objectives.

The Lost Accounts/Gained Accounts model focuses explicitly on how well a branch retains and attracts customers. The inputs for this model consist of the variables branches want to minimize (i.e. attritions) while the outputs consist of those things that branches want to maximize (i.e. funds, customers, products). The average CCR and BCC DEA scores were 0.73 and 0.81 , respectively. This model successfully identified best performers; however its target setting objectives were relatively unrealistic. To ameliorate this issue further, managerial insight and the application of multiplier restrictions are required. 
Market models measure the efficiency of branches relative to their local market conditions. For this study two Market models were constructed, one using individual product variables and the other using an aggregated metric. The results of both models were in agreement with published market model results; however, the aggregate model performed more similarly to literature than the component model. Moreover, the component model was found to have very poor discriminatory power, producing inconsequential results. The aggregate model offered much better discriminatory power and was deemed to be successful in attaining its objectives.

The final models examine a branch's ability to maximize changes in products, funds and customers. In total, two models were created and two differing methodologies were applied to each. Due to the nature of the data, the use of ratios and translations and the high sensitivity to outliers, these models were unable to produce acceptable results. They were thus regarded ineffective for the purpose of this study.

It was concluded that three of the six models constructed for this study are suitable for use in measuring the growth potential of bank branches. Further consideration and development of these models would aid in producing even more substantive results. The recommendations for future work include the acquisition of more detailed branch data over a several years and managerial input in designing multiplier restrictions for these models. 


\section{Chapter 1: Introduction}

The banking sector is one of the most crucial industries in both Canadian and global societies. It provides an avenue by which the government can exercise monetary control and supplies a means of regulating the lending and borrowing of assets. As was demonstrated by the 2007-8 economic collapse and ensuing economic difficulties, sound banking practices are essential in maintaining a country's economic health and stability. To ensure more stable economic environments, governments and global committees formulate regulations to govern banking activities. As a result, banks operate under very similar conditions and thus require adept customer service and marketing strategies to maintain or gain market share.

Even with the rapid advancement of technology, bank branches are still the main conduit through which banks handle transactions and funds. In Canada, the top five domestic banks alone possess upwards of 6150 bank branches. [CBA10] It is through this extensive network of branches that these banks are able to service their current customers and contact potential clients. It follows that a bank's marketability and growth capabilities are heavily reliant on their branch network as well as the individual growth potential of each branch. To successfully evaluate these criteria, banks must implement performance analysis and target setting at a branch level, rather than at an institutional level.

Due to its broad applicability, branch analysis is, in many cases, more desirable and important from a managerial stand point than institutional level analysis. It has the ability to provide information on branch performance that may lead to a better understanding of the variables and relationships that affect a bank's efficiency and profitability. [BERG97] Bank branches are also responsible for a large portion of the value added banking provided to customers and pose the highest operational expenses for a bank. Consequently, cost management can be more effectively performed at the branch level. Continuous improvement of branch performance, which can be achieved through the development and execution of DEA based strategies, is crucial in maintaining a competitive standing in the financial industry.

Despite the many attempts to accurately measure bank branch efficiency, the multifaceted nature of bank branches and the complexity of the services they provide have made this a difficult task. [KINS80] Financial ratios are unable to simultaneously consider all variables and are, therefore, unable to comprehensively describe branch performance. On the other hand, 
traditional profitability measures do offer some desirable characteristics, but comparative analysis of branches can be misleading. Their use of averages can make the identification of and comparison to top performers very difficult. Aside from the numerous profitability measures and financial ratios, banks use frontier efficiency analysis to objectively identify best practices within their organizations. [WU06a] Amongst the frontier efficiency analyses acknowledged in literature, Data Envelopment Analysis (DEA) was found to be one of the leading approaches in the banking industry.

Since its conception, the DEA methodology has made great theoretical advances, allowing for its application to a range of real world problems including those of the banking sector.[CHAR94],[SEIF96] Without the need for prior form specification of the production function, DEA provides an estimation of the production function to which each individual Decision Making Unit's (DMU's) efficiency score can be compared. Furthermore, DEA offers a number of advantages over traditional parametric techniques including its ability to identify reference units for each DMU. These characteristics prove to be a very useful managerial tool as it aids in establishing potential causes and methods of improvement for the identified inefficient DMUs. [EPST89]

Consequently, DEA has become one of the most widely used approaches to measure the efficiency of financial institutions.[BERG97] There exists a considerable number of papers on the use of DEA in the banking industry; however, the majority of these focus on banks at an institutional level.[PARA12] Institutional analysis has been most frequently used to assess the effects of ownership, bank type and regulatory and environmental changes, as well as bank performance and improvement, and international comparison. Whereas DEA branch analysis is applicable to a very broad range of business objectives, including the assessment of intermediation, cost, profitability, and resource allocation efficiencies and the identification of possible sources of inefficiency.

Of the aforementioned methods, the most commonly used DEA approaches to branch efficiency analysis are the production, intermediation and profitability approaches. The production approach measures a branch's production of transaction services (outputs) based on their capital and labour (inputs). The intermediation approach involves evaluating a branch's ability to make loans and investments (outputs) based on the monetary assets it has gathered (inputs). The profitability approach measures a branch's profitability based on expenses (inputs) and revenues (outputs). Alternatively, this study employs models which focus on assessing the 
client and market growth abilities of bank branches. To provide a multi-perspective evaluation several models have been formulated and their results compared.

\subsection{Problem Definition}

Since its more formal conception in the $14^{\text {th }}$ century, the banking industry has grown to be one of the most complex industries in the world. [DAVI02] Today's banks offer an extensive variety of products and services including simple chequing and savings accounts, mortgages and loans, retirement plans and mutual funds as well as various types of insurance. It follows that their list of competitors has grown to include insurance companies, credit unions, and lenders of last resort, government organizations and even virtual internet banks. In today's economic conditions, a bank's ability to remain globally competitive and continue growing its market share is heavily reliant on its ability to run efficiently and to properly identify its growth potential.

Due to the multifaceted nature of bank branches, measuring their efficiency is not a simple undertaking. Although there are many performance analyses currently used by banks, many of them produce conflicting results or lack the robustness required to fully appreciate the complexity of the relationships that exist. [PARA11] In an attempt to ameliorate these issues, this research focuses on developing multiple DEA models, each evaluating a unique perspective of branch growth. Through the subsequent comparison of results, true best performers and anomalies can be identified and a more comprehensive understanding of branch growth can be obtained.

One of Canada's major national banks, henceforth referred to as "Bank", currently employs various performance measures and statistical analyses to evaluate its branch network performance and to assess the growth potential of each branch. Through its analysis, the Bank attempts to determine the best available methods to gain market share and increase its Share of Wallet (SOW). The Bank uses the results from these analyses to determine resource allocation in branches and to help focus and implement marketing strategies. These results are also used in determining the location of new branches, as well as branch relocations and closures.

In planning to be able to better evaluate the growth potential of each branch in the Bank's branch network, a branch network Data Envelopment Analysis (DEA) was proposed. This analysis would involve several DEA models, whose individual foci include relative market efficiency, branch churn efficiency, and branch growth over a given year. In summation, the aim of this research is to provide the Bank with a more robust and comprehensive measure of bank branch efficiency as well as the ability to properly identify growth potential in branches. 


\subsection{Method OF APproach}

This study employs the now well accepted DEA methodology to evaluate the growth potential of the national branch network of one of Canada's major banks. This work involves the analysis of over a thousand branches and the implementation of multiple new and unique DEA models. The use of multiple base models allows for a more complete view of the inner workings of each individual branch and provides broader benchmarking capabilities. Moreover, this methodology allows managers to better assess which areas need the most improvement, providing an invaluable management tool.

The following chapters present the complete methodology used for this research; from theoretical conception to implementation and analysis.

- Chapter 2: Literature Review- provides a thorough review of relevant literature focusing on traditional performance analysis and frontier efficiency techniques used in the banking industry. A discussion of the uses of DEA in bank branch performance follows.

- Chapter 3: DEA- presents an overview of the history and development of the DEA models. In this section, various basic DEA models and formulations are discussed and some common extensions are introduced.

- Chapter 4: DEA Modeling- provides the methodology and models used to perform the analysis, as well as the results from each model.

- Chapter 5: Discussion of Results- presents the analysis and discussion of results and introduces additional statistical analysis and segmentation techniques used to investigate atypical results.

- Chapter 6: Conclusion and Recommendations- concludes the thesis; providing a brief synopsis of the completed work and an outline of the theoretical contributions of this research. Recommendations and directions of future work are also provided.

- Appendices- include various charts and graphs from the DEA analyses as well as all references and a glossary of terms. 


\section{Chapter 2: \\ Literature Review}

The banking industry is a central pillar of society, playing a vital role in both global and individual economic health. With the rapid advancement of technology, the banking sector has become increasingly competitive, requiring banks to continuously assess their management practices in order to maintain viability and growth. Traditional performance measures used by banks include financial ratios, indices and regression analysis, all of which can provide important insight and benchmarking capabilities. However, these methods have intrinsic limitations and myopic viewpoints that make them insufficient as standalone methods of analysis. [PARA11] As a more robust form of evaluation, banks use frontier efficiency analyses to objectively identify best practices within their organizations. Frontier methodologies are categorized as either parametric or non-parametric; with each offering unique advantages. Amongst the frontier efficiency analyses identified in literature, Data Envelopment Analysis (DEA), a non-parametric fractional linear programming technique, was found to be one of the leading approaches in the banking industry. In this chapter, a literature review on the performance analysis techniques used in the banking sector are presented. This includes a detailed discussion of the use of DEA for bank branch performance evaluations.

\subsection{Traditional Analysis Methods}

\subsubsection{RATIOS}

One of the most traditional and well-established performance analysis approaches used in the banking industry are Key Performance Indicators (KPI), more commonly known as financial ratios. These ratios offer the ability to measure the relationship between two numerical parameters, providing valuable insight into various aspects of banking including profitability, liquidity, asset quality and risk management. They also offer the desirable ability to quantify the change in parameter relationships over time. [GIOK08] Due to their simplicity and ease of computation, financial ratios, such as Return on Assets (ROA), Return on Equity (ROE) and Return on Investment (ROI), are still the most popular choice in many industry settings. [FRAS09]

Despite the widespread application of ratios for performance analysis, their use in the banking sector has many limitations. As a result of their simplistic nature, ratios are not very 
effective in assessing complex networks that perform multiple processes. [PARA04] Ratio analysis is inept in properly comparing significantly different branches or in simultaneously evaluating multiple components of a branch. [PARA11] Ratios are only capable of considering one input and provide only one output, precluding the ability to analyze situations where multiple inputs and outputs must be considered simultaneously (i.e. most banking operations). Ratios also suffer from inadequacies in assessing the effects of economies of scale, in providing benchmarks or in estimating overall performance measures of Decision Making Units (DMUs). Moreover, aggregating the results of a branch ratio analysis may result in misleading performance indicators and does not provide an objective means of determining whether a branch is actually efficient or not. [GIOK08]

\subsubsection{INDICES}

Index numbers are the most widely used method of measuring changes in economic variables over time. They are formulated using weighted ratios and thus can be customized to measure changes across firms, industries and countries. Index numbers that describe a number of diverse economic aspects are regularly compiled and published. These can include indices of import and export prices, price deflators for national income aggregates, and financial indices. The most commonly used economic index is Gross Domestic Product (GDP), which refers to the market value of all officially recognised final goods and services produced within a country in a given period of time. Banks employ several indices to help evaluate their branch performance. The Market Attractiveness Index, for example, compares the local population demographic with the local competitor base to determine which branches are operating in ideal environments. [COEL05]

Some of the earliest noted price indices were the Paasche and Laspeyres indices, which made their debut in the late nineteenth century. These indices are still commonly used today. Perhaps the most widely used price index is the Tornqvist index, which uses a basic geometric average of the current price of goods relative to base period prices. [IBRD04] Indices do, however, have some inherent weaknesses including the need to determine variable weights and their tendency to conceal deficient areas due to the aggregation of data. [PARA12]

\subsubsection{REgRESSION}

Regression analysis is a statistical tool that is used to investigate the relationship between variables. It is a parametric technique that requires the specification of a production function, 
also known as the regression function. The simplest form of regression analysis, single regression, is only capable of handling either multiple inputs and a single output or multiple outputs and a single input. More complex forms of regression analysis are capable of handling multiple inputs and outputs; however, these require the use of simultaneous regression functions. Whether utilizing single or multi-analysis, regression analysis only produces an estimate for either the input or output variables, depending on which is given. [SEN90]

Comparative studies between DEA and regression analysis ([BANK96], [BOWL85], [THAN93]) suggest that there is some agreement between the two methods, and that combining them may provide an alternative analysis opportunity. However, there are some clear advantages and disadvantages between each method. Regression analysis was found to be less prone to extreme inaccuracies at the individual DMU level. This characteristic is a result of its use of the full data set to obtain estimates, making them less sensitive to data fluctuations at the individual DMU level. Regression analysis was also found to be advantageous when setting individual maximum or minimum levels when the variables are independent of one other. [THAN93] Conversely, DEA was found to outperform regression analysis in identifying efficiencies and inefficiencies. Moreover, it is not always easy to identify the most suitable regression formula, and thus regression analysis requires a priori hypotheses, where DEA does not. [BOWL85]

\subsection{FrontiER EfFiciency Methods}

Due to the aforementioned limitations of traditional performance measures, frontier efficiency analyses have become preferred methods of evaluating performance. These methods objectively formulate an overall efficiency score for each unit, allowing for a sophisticated means of ranking units relative to one another. When dealing with more traditional, wellinformed industries, frontier efficiency methods will generally provide little qualitative information that is not already known. However, when dealing with complex service industries, such as the financial service industry, easily identifying maxima and minima is typically not possible. Frontier efficiency analysis provides these complex industries with the ability to quantify a unit's relative efficiency by comparing it with a known set of like units. These units can then be benchmarked against their peers, permitting management to objectively identify "best-practice" units. Moreover, these techniques allow for the identification of areas of input overuse and/or output underproduction.

Frontier efficiency methods can be divided into two categories; parametric and nonparametric techniques. The chief difference between the two techniques is that the functional 
form of the efficient frontier is pre-defined or imposed a priori in the parametric method, while the functional form is empirically calculated in the non-parametric approach. [MURI04] They also differ in the distributional assumptions imposed on their random error and inefficiencies. [BERG97] Despite these differences, parametric and non-parametric methods both offer their own advantages and disadvantages, and neither is strictly preferred over the other. A careful consideration of the techniques, available data set and industry characteristics should be performed to find the most suitable method of analysis. [MURI04], [BERG97]

Although most studies involving bank performance evaluation using frontier efficiency approaches focus on the application of one method, there are a few studies that provide a comparison between methods. The studies by Bauer et al. [BAUE93], Hasan and Hunter [HASA96], Berger and Mester [BERG97b] and Berger and Hannan [BERG98] provide comparisons of two or more parametric approaches. For the most part, these studies agreed that the average efficiencies obtained from each method were comparable and relatively consistent with normal banking conditions. Perhaps more relevant to this discussion are the studies of Ferrier and Lovell [FERR90], Eisenbeis et al. [EISE97], Resti [REST97], Bauer et al. [BAUE98] and Weill [WEIL04], which provide comparisons of parametric and non-parametric methodologies. Due to the inherent differences between the parametric and non-parametric methodologies, there were more inconsistencies found in these studies. However, the presence of these discrepancies was heavily reliant on the nature of the data and the function of the analysis.

There are five main frontier approaches identified in literature as methods to evaluate the efficiency of banks. Of these, there are three parametric methods, namely Stochastic Frontier Analysis (SFA), the Distribution-Free Approach (DFA) and the Thick Frontier Approach (TFA). The remaining two approaches, Free Disposal Hull (FDH) and Data Envelopment Analysis (DEA), are non-parametric methodologies.

\subsubsection{PARAMETRic Frontier ApProaches}

Parametric frontier approaches can be further subdivided into two categories deterministic and stochastic, both of which require prior specification of a functional form for the efficient frontier. Deterministic models, also termed "full frontier" models, envelope all of the observations and define technical efficiency as the distance between the observed data point and the frontier. Deterministic specification assumes all deviations from the efficient frontier can be modified by better managing the unit. It follows that this approach should not be used when the model includes uncontrollable variables. [MURI04] 
Stochastic frontier approaches differ from deterministic approaches in that they incorporate a double-sided random error into the specification of the frontier model. This allows the specification errors and uncontrollable factors to be modeled independently of the technical inefficiency component. [MURI04]

As with all modeling techniques, parametric methods offer some distinct advantages and disadvantages. The key advantage of the parametric models is that they allow for random error, thus reducing the chance of misidentifying error or confusing data as an inefficiency. The biggest challenge when employing a parametric method is accurately separating the random error from inefficiency. [BERG97] The chief disadvantage of the parametric approach is its inherent need for the pre-specification of the functional form. [BAUE98] The wrong form specification can lead to major inaccuracies in the resulting efficiency estimates. [GREB99] In the following sections, a brief discussion of the three main types of parametric frontier approaches are discussed; namely SFA, DFA and TFA.

\subsubsection{StOchastic FrontiER ANALYSis (SFA)}

One of the most commonly used parametric approaches is stochastic frontier analysis (SFA), also known as the econometric frontier approach. [HAO01], [BERG97a], [KAPA94] It was first introduced in 1977 when Aigner et al. [AIGN77], Battese and Corra [BATT77], and Meeusen and Van Den Broeck [MEEU77] worked simultaneously on the new method. SFA formulates a frontier for scenarios with either one input and multiple outputs or one output and multiple inputs. SFA allows for random error and assumes it follows a symmetric distribution, usually the standard normal distribution. The inefficiencies are assumed to follow an asymmetric distribution, generally the half-normal distribution. [BERG93] The use of different distribution models permits the separation and subsequent measurement of the random error and inefficiency.

SFA's assumption of a half-normal distribution for inefficiencies is fairly inflexible and presumes that most units cluster near full efficiency. Various studies ([GREE90], [YUEN93], [BERG97c]) have found that using the truncated normal or gamma distributions instead of the half-normal distribution gives additional flexibility to the evaluation of inefficiency. However, the similarity of these alternate distributions with that of the random error makes separation of the inefficiency from the random error term much more difficult. [BERG97]

\subsubsection{Distribution- FrEE APPROACH (DFA)}

The Distribution-Free Approach (DFA) ([BERG93], [DEYO94]) is similar to SFA in that it specifies the functional form of the frontier, but differs in its separation of random error and 
inefficiency. As long as the inefficiencies are non-negative, DFA allows them to follow nearly any distribution pattern. It assumes that the average efficiency of each unit remains constant over time while the random error averages to zero. DFA estimates each unit's inefficiencies by calculating the difference between the average residual of the data set and the average residual of the unit. Some truncation is then performed if the random error does not average to zero.

[BAUE98] DFA's assumptions suggest that efficiency does not fluctuate with time. Should fluctuations occur over time, DFA describes the average deviation of each unit from the average best practice frontier instead of the efficiency. [BERG97]

\subsubsection{THICK FrontIER APPROACH (TFA)}

The Thick Frontier Approach (TFA) ([BERG91], [CLAR96], [DEYO98]) specifies a functional form but imposes no distributional assumptions on either inefficiency or random error. TFA calculates separate cost functions for the highest and lowest cost quartiles for each class size. It then assumes that inefficiencies are represented by the difference between the highest and lowest quartiles and that the deviations within the highest and lowest quartiles represent random error. Therefore, units in the lowest average-cost quartile are assumed to have above average efficiency, forming a thick frontier. It should be noted that TFA does not provide efficiencies for individual units but overall efficiency, reducing the effects of extreme data points. [BERG97]

\subsubsection{Non-Parametric Frontier APPROACHES}

Unlike parametric techniques, non-parametric frontier approaches do not require prior specification of a functional form. This mitigates potential inaccuracy issues related to the preassignment of the functional form required in parametric methodologies. However, nonparametric methods are not without their disadvantages. Non-parametric approaches assume random error does not exist. This assumption can cause deviations in efficiency measures should a unit have associated random error. Furthermore, if this unit lies on the efficient frontier, the efficiencies of its peer units may also be affected. [BAUE98] In the following sections, a brief discussion of the main non-parametric technique, Data Envelopment Analysis (DEA), is provided along with an introduction to a specialized case of DEA termed the Free Disposal Hull (FDH).

\subsubsection{Data EnVelopment Analysis (DEA)}

Data Envelopment Analysis (DEA), first introduced by Charnes, Cooper and Rhodes in 1978 [CHAR78] extended Farrell's concept [FARR57] of estimating technical efficiency 
through the comparison of each organisational unit with the efficient Production Frontier. DEA is a non-parametric linear programming technique that is used to measure the relative efficiency of comparable Decision Making Units (DMUs). It provides an estimation of the empirical production function to which each individual DMU's efficiency score, ranging from 0 to 1 , can be compared. Furthermore, DEA offers a number of advantages over traditional parametric techniques including its ability to identify reference units for each DMU. This characteristic proves to be a very useful managerial tool as it aids in determining the potential causes and remedies for the identified inefficiencies. [EPST89] Additionally, DEA does not require prior assumptions on the specification of the production function's form or the distribution of the observations. A detailed discussion of the history, theory and mathematical formulation of basic DEA models are provided in Chapter 3.

\subsubsection{Free Disposal Hull (FDH)}

The Free Disposal Hull (FDH) approach ([DEPR84], [TULK93], [FRIE96], [DEKK01]) is a variation of DEA where the efficient frontier includes only the DEA vertices and the free disposal hull points interior to these vertices. [BERG97] Rather than DEA's piecewise linear frontier, FDH constructs a stepwise frontier so that efficiency measures are affected only by actual observations. [COOP07] As a result of the FDH frontier being either congruent with or interior to the DEA frontier, the FDH methodology generally produces higher estimations of average efficiency than DEA. [TULK93]

\subsection{DEA in Bank Branch Performance Evaluation}

Bank branch analysis is in many cases more desirable and informative than institutional level analyses. Branch analyses help in understanding the complex relationships and variables that can play a role in bank level efficiency. These analyses also resolve measurement problems that exist in standard bank level analysis. [BERG97] Furthermore, the bank's largest operational expenses are usually realized at the branch level. Consequently, branch analysis can provide an effective managerial tool and a more direct cost management solution. Lastly, branches are the source of a large portion of the value added banking performed by customers, who, in these tough economic times, are looking for reliable face to face contact with their bank. [BCG10] Branch analysis can provide great insight into branch operations, leading to more sound and comprehensive branch strategies. If properly executed, these strategies can provide the 
improvement and branch network growth that is necessary to remain competitive in the financial services marketplace.

Since its conception, DEA has become one of the most widely used approaches to measure the efficiency of financial institutions. [BERG97] However, the majority of DEA banking studies have focused on banks at an institutional level, rather than branch level analyses. This can partially be attributed to the difference in data availability. The majority of banks are publicly traded on major exchanges and thus, must provide their investors with quarterly and annual financial reports. This makes the collection of data for institutional level analyses rather easy. On the contrary, branch level data is proprietary information and is not generally disclosed to the public. Instead, it is either amassed into bank financial reports or not reported at all. Nonetheless, surveys have shown that there has been a steady increase in DEA branch studies, nearly doubling in the last five years alone. [PARA12]

To date, there are four survey papers that review DEA applications in the banking industry, of which three focus on bank level applications and one focuses on branch level applications. The first to review the major efficiency techniques used in the evaluation of bank performance were Berger and Humphrey. [BERG97] This survey reviewed a total of 103 papers including 57 DEA based papers. Of these, 42 focused on bank level analysis while the remaining 15 focused on branch level analysis. Berger [BERG07] provided a review and critique of over 100 studies that compared cross-national bank efficiencies obtained using various frontier techniques. Fethi and Pasiouras [FETH10] presented a review of 196 studies which employed operational research and artificial intelligence techniques to assess bank performance. Of these studies, 151 of them used DEA-like techniques and 30 focused on evaluating efficiency at a branch level. Most recently, Paradi and Zhu [PARA12] published a survey that focused heavily on the use of DEA in branch analysis. Among the 285 bank related DEA publications identified, 90 focused on branch analysis and were discussed in greater detail.

This section summarizes the key DEA model types and objectives used in branch level analyses. A brief introduction to a few common of model variations is also provided. It should be noted that this study evaluates the branch network of one of the top five Canadian banks by applying certain models and extensions discussed herein.

\subsubsection{MODEL OBJECTIVES}

Branch level DEA applications can have a diverse set of business objectives; however, the majority of applications focus on evaluating branch specific operations. These studies allow 
for the exploration of efficiency determinants and provide the capability of identifying deficiencies in areas that are controllable by branch managers. That being said, branch performance measurement is not a simple task. Branches come in an assortment of sizes, operate in different economic regions and offer a variety of services to a diverse range of customers. In order for a branch performance analysis to be significant and reliable, it should capture the critical aspects of the bank's internal operating processes, leading to a more adept understanding of these processes. Moreover, the analysis should provide target setting through the identification of best- and worst-practices and offer the capability of investigating the sources of the inefficiencies.

Depending on the objective of the analysis, different DEA model frameworks exist. Of these models, there are three that are very commonly used in branch analysis; intermediation, production, and profitability. Additionally, the market model, employed in this study, is introduced below.

\subsubsection{INTERMEDIARY MODEL}

The intermediation approach was the earliest DEA model used to assess the performance of banks. [COLW92] It evaluates the branch's ability to collect deposits and other funds (inputs) and then lend the money in various forms, including loans, mortgages and other assets (outputs). An early example of this approach is found in Alhadeff's study (1954) that measured output in terms of dollar values of earning assets. [COLW92]

There are relatively few branch analyses that employ the intermediation approach. The first study, done by Athanassopoulos, used non-interest and interest costs as inputs and noninterest income and total volume of accounts for loans, savings and deposits for outputs. [ATHA97] Subsequently, Athanassoupoulos developed a two stage model whose first stage evaluated service efficiency. The output targets produced by this stage were then used as the inputs into the second-stage intermediation model. This modified intermediary model produced a larger efficient frontier, providing each DMU with more room for improvement. [ATHA00]

\subsubsection{PRODUCTION MOdEL}

The production model, first used for branch analysis in 1985 by Sherman and Gold [SHER85], is the most popular approach for bank branch performance analysis. It views the bank branches as producers of services and products, using labour and physical resources (inputs) to produce transactions, such as loans and deposits (outputs). The transactions considered can consist of face-to face interactions at the branch, back office transactions and delivered 
transactions. Consideration of customer satisfaction has also been found to play a role in the production efficiency of a branch. There have been numerous studies that have employed this methodology, some examples of which include Parkan [PARK87], Schaffnit et al. [SCHA97], Athanassopoulos [ATHA98], Camanho and Dyson [CAMA05], Portela and Thanassoulis [PORT07], Giokas [GIOK08],Tsolas [TSOL10] and Paradi et al. [PARA11].

\subsubsection{Profitability Model}

The profitability model is another commonly used method of bank branch analysis. It measures the branch's ability to use labour, assets and capital to generate profits, or more simply put, the branch's ability to convert expenses into revenues. The expenses (inputs) include employee expenses, occupancy expenses, branch cross charges, and other operational expenses. Additionally, loan losses and sundry can be included on the input side to penalize those branches with higher losses, whether from risky lending or unwanted revenue charges. The branch's revenues (outputs) are based on all of the branch's lines of business: non-interest revenues and bank fees, interest earning from wealth management, home mortgages, consumer lending, consumer deposits, commercial lending and commercial deposits, and commission revenues earned through wealth management, credit cards and insurance brokerage. Some examples of studies employing bank branch profitability analysis are Manandhar and Tang [MANA02], AlTamini and Lootah [ALTA07], McEachem and Paradi [MCEA07], Paradi et al. [PARA10],[PARA11] and Tsola [TSOL10].

\subsubsection{MARKET MODEL}

The market model is relatively uncommon among bank branch efficiency analyses. It is, however, very well suited for the requirements of this study and for the analysis of growth potential. Market efficiency was first defined by Athanassopoulos [ATHA95] in a study related to retail organizations. The market model measures the extent to which a bank branch, given its capacity and available resources, realizes its potential to sell products and provide services in a given market. The main objective of a bank branch is to penetrate its market by selling financial products to new customers while continuing to deliver services to existing customers. To achieve market efficiency, a bank branch must expand their outputs and optimize how cohesively the branch (size, number of employees, etc.) fits into its market conditions. Examples of market models applied to bank branch efficiency analysis include Athanassopoulos [ATHA98], Thanassoulis [THAN99] and Athanassopoulos and Giokas [ATHA00]. 


\subsubsection{MODEL VARIATIONS}

DEA offers a wide range of model variations that must be carefully considered when building a model for a particular bank branch analysis. Inevitably, modellers must determine which specific DEA version to apply and which extensions are necessary to properly evaluate the given data and meet the analysis objectives. In this section, several model variations are introduced as well as relevant extensions that were used in this study.

\subsubsection{RETURNS TO SCALE}

In bank branch efficiency analysis, DEA can be employed assuming either constant returns to scale (CRS) or variable returns to scale (VRS). The first DEA model introduced by Charnes et al. [CHAR78] was a CRS model referred to as the CCR model. This model has been used extensively in research, accounting for nearly half of all bank branch efficiency studies. [PARA12] McEachrn and Paradi [MCEA07] state that when commercial and specialty branches, such as oil and gas, and real estate branches, were excluded, branches operate on a CRS basis. Fethi [FETH10] on the other hand suggests that CRS models are only suitable when all DMUs are operating at an optimal scale. Approximately $30 \%$ of branch analyses employ the BCC model, a VRS approach. Cook et al. [COOK00] examined over 1300 Canadian branches using the VRS approach. Camanho and Dyson [CAMA05] claimed that the efficient frontier should be estimated assuming VRS for the production model and CRS for the value-added approach. The remaining 20 or so percent of branch studies employed both CRS and VRS methods in their analysis. Ultimately, determining the most suitable model form is dependent on both the data set and the analysis objectives.

\subsubsection{INPUT ORIENTED VS. OUTPUT ORIENTED}

DEA can estimate efficiency under either an input-oriented or output-oriented approach. The input-oriented approach determines by how much input quantities can be reduced without changing output quantities. Conversely, the output-oriented approach determines by how much output quantities can be increased without changing input quantities. Branch managers tend to have more control over the inputs (labour and capital) than the outputs (profit, loans and transactions) and thus, input oriented approaches are more commonly used in branch performance efficiency studies. It should be noted that there also exists a more complex nonoriented approach called the slack-based measure that looks to simultaneously decrease inputs and increase outputs. [FETH10] 


\subsubsection{Multi-StAgE DEA AnAlysis}

In the business world, it is not uncommon to witness managers employing unilateral strategies that are purely in response to the head office's assessment criteria. This improvement approach tends to lead to decreased efficiency in areas not accounted for in the strategy. Bank branches are complex, multi-faceted organizations that require careful assessment and personalized strategies. In attempts to provide a more comprehensive performance measure, several researchers have simultaneously applied more than one DEA model to evaluate overall branch efficiency. Moreover, this methodology provides better target setting opportunities. Examples of multi-level DEA models used for branch analysis include Sherman and Ladino [SHER95], Manandhar and Tang [MANA02], Jahanshahloo et al. [JAHA04], Al-Tamimi and Lootah [ALTA07], Portela and Thanassoulis [PORT07], Giokas [GIOK08] and Paradi et al. [PARA11].

\subsubsection{RELEVANT EXTENSIONS}

When performing a branch efficiency analysis using DEA, there are several model extensions that are required in order to produce realistic and attainable targets. Of these extensions, restricted multiplier models, non-discretionary variables and fractional data are introduced herein. The theoretical properties of these extensions are discussed in more detail in Chapter 3.

\section{Restricted Multiplier Model}

As previously mentioned, DEA does not require a priori knowledge of the functional form or of the variables used. Although this is a desirable characteristic, it can potentially result in solutions with variable multipliers that are not realistically feasible. In these situations, it can be very useful to employ multiplier restrictions. This extension proves very valuable from a managerial stand point as it provides managers with the opportunity to limit the changes made to any specific variable. Examples of restricted multiplier models applied to bank branch analyses include Schaffnit et al. [SCHA97], Cook and Hababou [COOK01], Paradi and Schaffnit [PARA04] and Paradi et al. [PARA11].

\section{Non-Discretionary Variables}

There are numerous instances when a variable is highly pertinent to the evaluation of a DMU but cannot be controlled by management. These variables, referred to as non-discretionary 
variables, can include anything from weather to population demographics to branch square footage. The very nature of these variables prevents their alteration or manipulation, and thus, they cannot be treated in the same manner as discretionary variables. To avoid unachievable recommendations from the DEA model, non-discretionary variables are generally removed from the objective function in the linear programming model, but are included in the constraints so that their presence is still accounted for when evaluating the DMUs. Non-discretionary variables are very common in all types of DEA efficiency analysis including bank branch analysis. Some more recent studies employing non-discretionary variables in branch analysis include Paradi and Schaffnit [PARA04], Wu et al. [WU06b], Portela and Thanassoulis [PORT07] and Paradi et al. [PARA11].

\section{Fractional Data}

When evaluating banks and bank branches, there are many cases where the most readily available data is in the form of ratios. In order to use this data to create a successful DEA model, one must keep in mind a few key points. Firstly, the use of ratios leads to the loss of information about the size of a unit, and implicitly assumes constant returns to scale in the operation of the DMUs in question. Many of these performance indicators use different denominators, and thus, have the virtue of being independent of size. The ratio approach will not lead to major difficulties, provided that the process of assigning multipliers is closely monitored. They should continue to represent the value of a unit increase in one ratio relative to a unit increase in another. However, if the ratio form of DEA is used, then it is crucial that the BCC form be implemented instead of the CCR form. [HOLL03] 


\section{Chapter 3: \\ Data Envelopment Analysis (DEA)}

This chapter presents an overview of the analysis technique, Data Envelopment Analysis (DEA), used for this bank branch study. To begin, a brief history and background of the technique is presented, followed by theory and the mathematical formulations of DEA. Basic DEA models are introduced, as well as relevant extensions to these models. The chapter concludes with a discussion of the results obtained from DEA and the strengths and limitations of this technique.

\subsection{DEA: A HISTORY}

Introduced in 1978, Data Envelopment Analysis extended Farrell's concepts of technical and allocative efficiency. Inspired by Debreu [DEBR51] and Koopmans [KOOP51], Farrell [FARR57] proposed that the efficiency of a firm, termed as overall efficiency, could be separated into technical and allocative efficiency. Technical efficiency represented a firm's ability to maximize output given a set of inputs, while allocative efficiency denoted the firm's ability to optimally employ inputs given their price and the firm's production capabilities. [COEL98] Farrell's concept used simple ratios of a single input over a single output to measure efficiencies. He demonstrated his ideas under the restriction of linear homogeneity, forcing constant returns to scale and limiting the versatility of the technique. Furthermore, Farrell's technique is a radial measure and measures technical efficiency relative to an isoquant; two characteristics that can lead to erroneous efficiency measures. [FARE78]

In an attempt to ameliorate these issues, Farrell and Fieldhouse [FARR62] attempted to circumvent the constant returns restriction by grouping data and adjusting for output levels. The method was based on an index of multiple inputs and outputs, where weights were assigned to all inputs and outputs for each unit measured. However, assigning a common weight to all of the units under consideration proved to be very difficult. [FARE85]

Subsequent to Farrell and Fieldhouse' new method, alternative linear production models with looser linear homogeneity restrictions were introduced. Amongst these models was the revolutionary Data Envelopment Analysis model proposed by Charnes, Coopers and Rhodes. [CHAR78] The DEA methodology proposed allowing each Decision Making Unit (DMU) to choose its own variable weights/multipliers. In turn, this resulted in each DMU looking as 
favourable as possible to its peers. This characteristic allowed DEA to be used when assigning numerical values to variables proved difficult or when variables were qualitative in nature. Although originally developed for use in non-profit and governmental organizations, DEA has found applications in many areas of study. It has also been subject to numerous theoretical advances and methodological extensions. Most notably was the development of the BCC model by Banker et al. [BANK84], which allowed for variable returns to scale. Some other noteworthy extensions pertinent to this study include the use of categorical and non-discretionary variables, the constricted multiplier model and the Slack-Based Model (SBM). Further discussion of these extensions is provided in this chapter.

\subsection{DEA THEORY AND MATHEMATICAL FORMULATION}

DEA is a linear programming technique that defines the set of best-practice or frontier observations as those for which no other DMU or linear combination of DMUs has as much or more of every output for as much or less of every input. DEA produces a convex production possibilities set by connecting the best-practice observations with a piecewise linear frontier. All best-practice DMUs sitting on the frontier are considered efficient and receive an efficiency score of 1 . The units not on the frontier are considered inefficient and receive an efficiency score of less than 1 . These scores are calculated by projecting the inefficient unit onto the efficient frontier. DEA also provides benchmarking and target setting capabilities for the inefficient units, allowing management to better recognize best practices and implement improvement strategies. [BERG97]

In order to successfully execute DEA to produce meaningful efficiency scores, there are some important data criteria that should be met. Firstly, DEA evaluates inefficient DMUs by comparing them to the best-practice observations; therefore, it is crucial that all DMUs are, in fact, comparable. It follows that all DMUs must operate in the same cultural environment. As previously noted, DEA does not account for random error and the efficient frontier is very sensitive to measurement error, thus, data should be carefully cleaned and all irregularities should be removed. [BERG97] Moreover, for a DMU to be properly evaluated it must have a complete set of data for all inputs and outputs.

When performing a DEA analysis, one must also pay attention to the number of Degrees of Freedom (DOF) of the model. The DOF increases with the number of DMUs and decreases with the number of input and output variables. A general rule for the minimum number of DMUs (n) is that it should exceed the greater of either: three times the sum of the number of inputs (m) 
and outputs $(\mathrm{s})$ or the product of the number of inputs $(\mathrm{m})$ and outputs $(\mathrm{s})$. [COOP07] This rule is shown below:

$$
n \geq \max \{m \times s, 3(m+s)\}
$$

Lastly, when building a DEA model, one must carefully choose variables that are appropriate for the model's objectives and that properly represent the process that is being evaluated. Statistical analysis should also be completed to remove all redundancies within the variables.

The DEA methodology is based on the measure of a DMU's efficiency. In general, efficiency is measured using a ratio of outputs/inputs. This can be denoted mathematically as:

$$
\text { Efficiency Score of } D M U_{0}=\operatorname{Max} \frac{\text { output }_{0}}{\text { input }_{0}}=\operatorname{Max} \frac{\sum_{r=1}^{s} u_{r} y_{r 0}}{\sum_{i=1}^{m} v_{i} x_{i 0}}
$$

Where $\quad y_{r j}$ : the quantity of the $\mathrm{r}^{\text {th }}(\mathrm{r}=1,2, \ldots, \mathrm{s})$ input for unit $\mathrm{j}(\mathrm{j}=1,2, \ldots, \mathrm{n})$

$u_{r}$ : the weight associated with the $\mathrm{r}^{\text {th }}$ input variable

$x_{i j}$ : the quantity of the $\mathrm{i}^{\text {th }}(\mathrm{i}=1,2, \ldots, \mathrm{s})$ output for unit $\mathrm{j}$

$v_{i}$ : the weight associated with the $\mathrm{i}^{\text {th }}$ output variable

The greater the ratio, the higher the efficiency; thus, efficiency improvement can be obtained by increasing the outputs or decreasing the inputs.

\subsection{Constant Returns-To-Scale (CRS) Model}

Introduced by Charnes, Cooper and Rhodes, the Constant Returns-to-Scale (CRS) model, also referred to as the CCR model, was the first formulated DEA model. [CHAR78] As the name implies, this model was built on the assumption that the analysed units are operating under constant returns-to-scale meaning that, regardless of operation scale, increases in inputs result in proportional increases in outputs. The CRS model assigns weights $(\mathrm{u}, \mathrm{v})$ to each input $\left(\mathrm{X}_{\mathrm{j}}=\left\{\mathrm{x}_{\mathrm{ij}}\right\}\right)$ and output $\left(\mathrm{Y}_{\mathrm{j}}=\left\{\mathrm{y}_{\mathrm{rj}}\right\}\right)$ variable in order to maximize each unit's relative efficiency score $(\theta)$, with no score exceeding one. Essentially, the DEA model is attempting to make each DMU look as favorable as possible.

The CCR model provides a measure known as the overall technical efficiency, which aggregates both technical efficiency and scale efficiency. Efficient DMUs have an efficiency score of one with both slacks from the efficiency $\left(\mathrm{s}^{-}\right.$and $\left.\mathrm{s}^{+}\right)$being zero, while inefficient DMUs have efficiency scores that range from 0 to 1 . Additionally, DMUs can be termed as weakly efficient if there are slacks present and their efficiency score is one.

The CCR model has the following fractional formulation: 


$$
\text { Maximize } \quad h_{0}=\frac{u_{1} y_{10}+u_{2} y_{20}+\cdots+u_{s} y_{s 0}}{v_{1} x_{10}+v_{2} x_{20}+\cdots+v_{m} x_{m 0}}
$$

Subject to:

$$
\begin{aligned}
& \frac{u_{1} y_{1 j}+\cdots+u_{s} y_{s j}}{v_{1} x_{1 j}+\cdots+v_{m} x_{m j}} \leq 1(j=1, \ldots, n) \\
& u_{1}, u_{2}, \ldots, u_{s} \geq \varepsilon \\
& v_{1}, v_{2}, \ldots, v_{m} \geq \varepsilon
\end{aligned}
$$

Where: $\quad x_{i j}=$ the amount of the $i^{\text {th }}$ input to unit $j$

$\mathrm{Vi}=$ the weight given to the $\mathrm{i}^{\text {th }}$ input

$\mathrm{y}_{\mathrm{rj}}=$ the amount of the $\mathrm{r}^{\text {th }}$ output from unit $\mathrm{j}$

$\mathrm{ur}_{\mathrm{r}}=$ the weight given to the $\mathrm{r}^{\text {th }}$ output

This ratio model evaluates the relative performance of the DMUs given the observed performance of the production possibilities set. The denominator represents a weighted sum of ' $\mathrm{m}$ ' inputs used by the DMU to produce a weighted sum of ' $\mathrm{s}$ ' outputs represented by the numerator. Each DMU is assigned unique multipliers to give the DMU the highest efficiency score possible while ensuring that no other DMU's score exceeds one. To prevent any of the assigned multipliers from being zero or negative, $\varepsilon$, a non-Archimedean constant having a value smaller than any positive valued real number, is used as a constraint in the model. [COOP07]

A graphical representation of the CCR model is presented below in Figure 3.1. The CCR efficient frontier begins at the origin and continues as a straight line. This frontier consists solely of efficient units enveloping the remaining inefficient DMUs. As is shown, the only efficient DMU in this data set is DMU H; hence, it is the only DMU on the frontier. The remaining DMUs are all considered inefficient.

The CCR model can either be input oriented or output oriented. Both of these CCR models are scale invariant, allowing for variable adjustment, and unit invariant, allowing variables with differing units to appear in the same model. However, neither CCR model is translation invariant as the frontier must pass through the origin. Moreover, scalar changes to the variables in either CCR model will result in a change in slope of the frontier, thus altering the efficiency scores of the DMUs. In the following sections, the input-oriented and the outputoriented CCR models are introduced using a less computationally intensive linear programming model derived from the aforementioned fractional CCR model. 


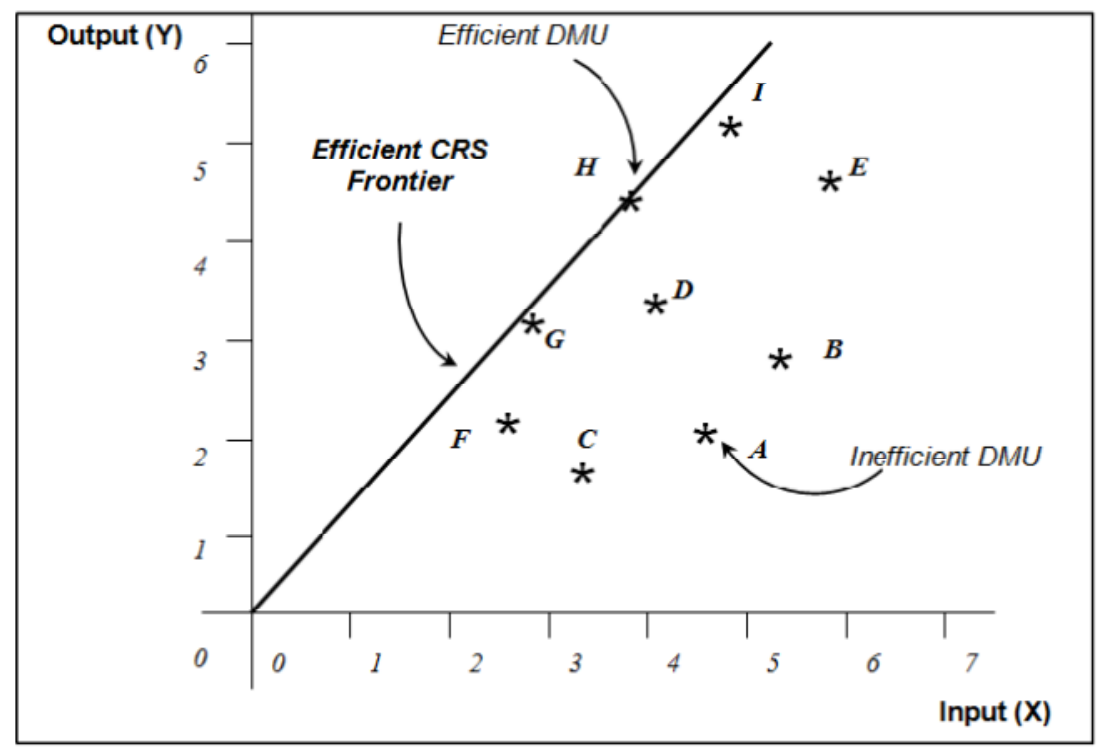

Figure 3.1: Graphical Representation of the CCR Model [MIN01]

\subsubsection{INPUT ORIENTED CRS MODEL}

The input-oriented CCR model aims to minimize inputs for the same observed output. This CRS model has both a primal and a dual form. To develop the primal linear programming model (3.4), the denominator of the CCR ratio model is used as the normalizing constraint, while the numerator becomes the objective function. The dual linear programming model (3.5) assigns a dual variable to each constraint in the primal model. This model is carried out in two stages; first the radial efficiency is calculated, followed by the mix efficiency.

\section{Primal CCR Input-Oriented Model (3.4)}

Maximize: $\quad w_{0}=\sum_{r=1}^{s} u_{r} y_{r 0}$

Subject to

$$
\begin{aligned}
& \sum_{r=1}^{s} u_{r} y_{r j}-\sum_{i=1}^{m} v_{i} x_{i j} \leq 0 \\
& \sum_{i=1}^{m} v_{i} x_{i 0}=1 \\
& -u_{r} \leq-\varepsilon \\
& -v_{i} \leq-\varepsilon
\end{aligned}
$$

Dual CCR Input-Oriented Model (3.5)

Minimize: $\quad z_{0}=\theta-\varepsilon\left[\sum_{i=1}^{m} s_{i}^{-}+\sum_{r=1}^{s} s_{r}^{+}\right]$

Subject to

$$
\begin{aligned}
& y_{r 0}=\sum_{j=1}^{n} y_{r j} \lambda_{j}-s_{r}^{+} \\
& 0=\theta x_{i 0}-\sum_{j=1}^{n} x_{i j} \lambda_{j}-s_{i}^{-} \\
& 0 \leq \lambda_{j}, \text { for } \mathrm{j}=1, \ldots, \mathrm{n} \\
& 0 \leq s_{i}^{-}, \text {for } \mathrm{i}=1, \ldots, \mathrm{m} \\
& 0 \leq s_{r}^{+}, \text {for } \mathrm{r}=1, \ldots, \mathrm{s}
\end{aligned}
$$

The dual model introduces some variables that were not present in the fractional form. The $\theta$ variable represents the proportional input reduction applied to the DMU under consideration. This reduction is applied to all of the DMU's inputs in order to bring the unit 
closer to the efficient frontier. The $\theta$ variable is constrained between zero and one, with one representing full efficiency or radial efficiency. This variable also denotes the required percent of inputs inefficient DMUs would required to theoretically produce the same amount of outputs. Furthermore, the dual form employs a set of non-negative intensity variables, $\lambda$, that represent the weight of each of the $n$ DMUs.

The presence of the non-Archimedean constant ensures that the mix efficiency is calculated after the radial efficiency by assigning the slacks a lower level of importance. It follows that once the model has calculated the radial efficiency of the DMU it will then calculate the input and output slack variables, also referred to as input excesses, $\mathrm{s}_{\mathrm{i}}{ }^{-}$, and output shortfalls, $\mathrm{S}_{\mathrm{r}}{ }^{+}$. If slacks are present, the DMU is considered to have mix inefficiencies. Thus, it will require further reductions beyond the optimal $\theta^{*}$ reductions found in stage one and the input proportions will need to be adjusted. If both slacks are zero and $\theta^{*}$ is equal to one, then the DMU is considered fully efficient. As previously mentioned, if $\theta^{*}$ is equal to one with non-zero slacks, then the DMU is considered radially efficient with mix inefficiencies, or weakly efficient.

The input-oriented CCR model provides target setting for inefficient DMUs by referring these units to the efficient frontier formed by the DMU`s unique reference set of efficient DMUs denoted as $\mathrm{E}_{0}$. As shown in formulas (3.6) and (3.7), $\mathrm{DMU}_{0}$ is projected to point $\left(\hat{x}_{0}, \hat{y}_{0}\right)$ on the frontier, where $\left(\hat{x}_{0}, \hat{y}_{0}\right)$ are the coordinates of a virtual linear composite used to evaluate the performance of $\mathrm{DMU}_{0}$. Moreover, $\left(\hat{x}_{0}, \hat{y}_{0}\right)$ denotes the theoretically feasible efficient production that $\mathrm{DMU}_{0}$ should strive for.

$$
\begin{array}{ll}
\hat{x}_{i 0}=\theta^{*} x_{i 0}-s_{i}^{-*} & (\mathrm{i}=1, \ldots, \mathrm{m}) \\
\hat{y}_{r 0}=y_{r 0}+s_{r}^{+*} & (\mathrm{r}=1, \ldots, \mathrm{s})
\end{array}
$$

\subsubsection{OUTPUT ORIENTED CRS MODEL}

The output-oriented CCR model aims to maximize outputs while utilizing the same amount of observed inputs. Much like the input-oriented model, the output-oriented model has both a primal and dual form as shown below: 


\section{Primal CCR Output-Oriented Model}

Minimize: $\quad \sum_{i=1}^{m} p_{i} x_{i 0}$

$$
\begin{aligned}
& \text { Subject to } \quad \sum_{r=1}^{s} q_{r} y_{r j}-\sum_{i=1}^{m} p_{i} x_{i j} \leq 0 \\
& \sum_{r=1}^{s} q_{r} y_{r 0}=1 \\
& -p_{i} \leq-\varepsilon \\
& -q_{r} \leq-\varepsilon
\end{aligned}
$$

Dual CCR Output-Oriented Model (3.9)

Minimize: $\quad \eta-\varepsilon\left[\sum_{i=1}^{m} t_{i}^{-}+\sum_{r=1}^{s} t_{r}^{+}\right]$

$$
\begin{array}{ll}
\text { Subject to } & x_{i 0}=\sum_{j=1}^{n} x_{i j} \mu_{j}+t_{i}^{-} \\
& 0=\eta y_{r 0}-\sum_{j=1}^{n} y_{r j} \mu_{j}+t_{r}^{+} \\
& 0 \leq \mu_{j}, \text { for } \mathrm{j}=1, \ldots, \mathrm{n} \\
& 0 \leq t_{i}^{-}, \text {for } \mathrm{i}=1, \ldots, \mathrm{m} \\
& 0 \leq t_{r}^{+}, \text {for } \mathrm{r}=1, \ldots, \mathrm{s}
\end{array}
$$

This model functions in the same manner as the input-oriented CRS model. Much like the $\theta$ variable, the $\eta$ variable in the output-oriented model represents the output increase applied to the DMU under consideration. This increase is applied to all of the DMU's outputs in order to bring it closer to the frontier. Again, like the $\theta$ variable, the $\eta$ variable is constrained between zero and one, with one representing full efficiency or radial efficiency. If a DMU is inefficient under the output-oriented model, the $\eta$ variable represents the percent increase in outputs the DMU could theoretically produce given the same amount of inputs. Similar to the input-oriented model, the output-oriented dual form employs non-negative intensity variables, $\mu$, that represent the weight of each of the $n$ DMUs.

An optimal solution for the output-oriented dual model can be derived from the optimal solution of the input-oriented model using the following relations:

$$
\lambda=\mu / \eta \quad \theta=1 / \eta
$$

Moreover, the slacks $\left(\mathrm{t}^{-}, \mathrm{t}^{+}\right)$of the output oriented model can be related back to the input oriented model through its optimal solution:

$$
t^{-*}=s^{-*} / \theta^{*}, \quad t^{+*}=s^{+*} / \theta^{*}
$$

As with the input-oriented model, DMUs in an output-oriented model are fully efficient if and only if $\eta^{*}$, the optimal expansion from the first phase, is equal to one and all optimal slacks are zero. For target setting purposes, inefficient DMUs can be projected to the efficient frontier using the following formulation:

$$
\begin{array}{ll}
\hat{x}_{i 0}=x_{i 0}-t_{i}^{-*} & (\mathrm{i}=1, \ldots, \mathrm{m}) \\
\hat{y}_{r 0}=\eta^{*} y_{r 0}+t_{r}^{+*} & (\mathrm{r}=1, \ldots, \mathrm{s})
\end{array}
$$




\subsection{VARIABLE RetURNS-To-ScAle (VRS) MODEL}

The Variable Returns to Scale (VRS) Model or BCC model, first introduced in 1984 by Banker, Charnes and Cooper, allows more flexibility than the CCR model by providing a variable returns-to-scale DEA formulation. [BANK84] As is shown in Figure 3.2, the BCC frontier does not cross through the origin as did the CCR model, but has a piecewise linear frontier that is convex in shape. This frontier, comprised of best performing DMUs, encapsulates the inefficient DMUs.

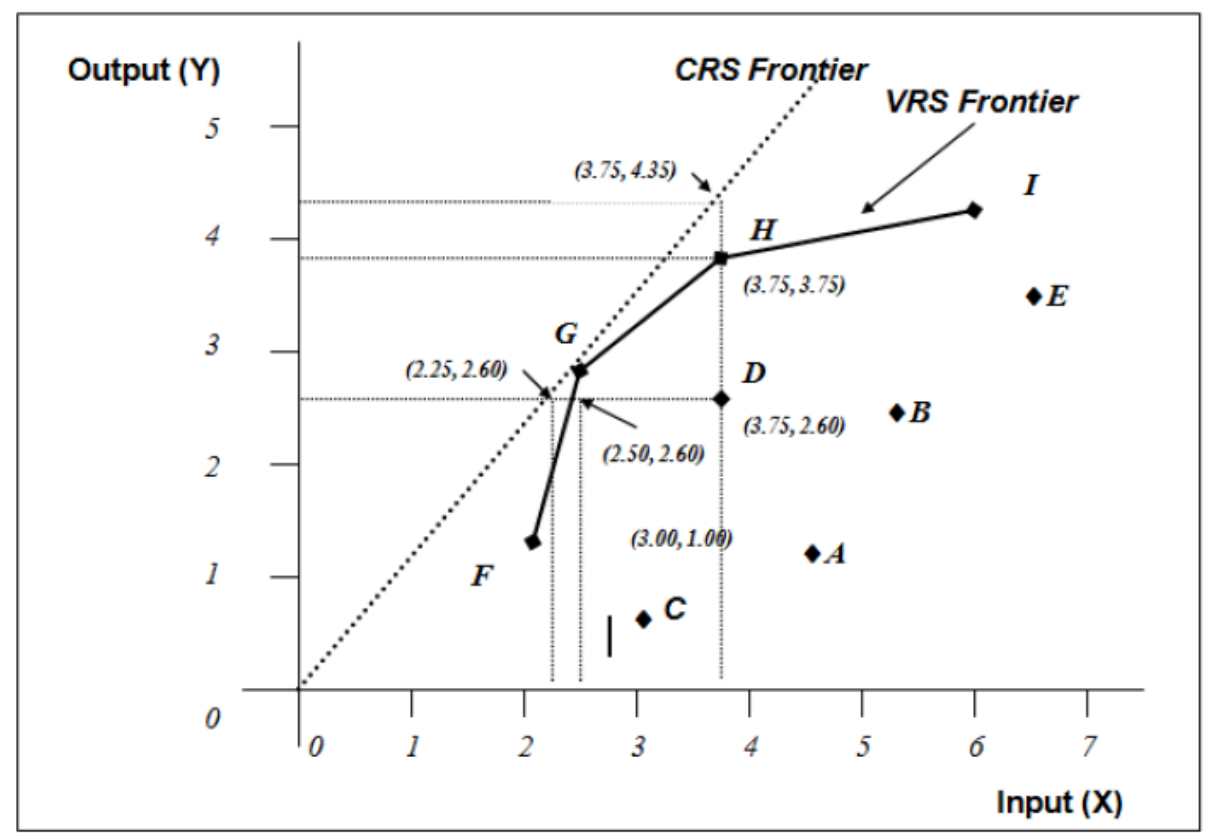

Figure 3.2: Graphical Representation of BCC Model [MIN01]

The BCC model is formulated in much the same way as the CCR model with the exception of the addition of the $\tilde{u}_{o}$ variable, which accounts for economies of scale. Given a unique optimal solution is found: $\tilde{u}_{o}<0$ indicates that the units are operating under increasing returns-to-scale, $\tilde{u}_{o}=0$ indicates constant returns-to-scale and $\tilde{u}_{o}>0$ indicates decreasing returns-to-scale. It should also be noted that although the $\tilde{u}_{o}$ variable estimates the scale economies, the BCC model measures technical efficiency alone and does not account for scale inefficiencies. [COOP07]

As with the CCR model, the BCC model can be either input-oriented or output-oriented. The following sections provide an introduction to these two models, outlining their formulations and target setting techniques. 


\subsubsection{INPUT ORIENTED VRS MODEL}

The formulation of the input-oriented VRS model maximizes the efficiency scores of $n$ units. The fractional VRS model is depicted below:

$$
\text { Maximize } \quad \theta=\frac{\sum_{r=1}^{S} u_{r} y_{r 0}-\widetilde{u}_{0}}{\sum_{i=1}^{m} v_{i} x_{i 0}}
$$

Subject to: $\quad \frac{\sum_{r=1}^{S} u_{r} y_{r j}-\widetilde{u}_{0}}{\sum_{i=1}^{m} v_{i} x_{i j}} \leq 1(j=1, \ldots, n)$

$$
\begin{aligned}
& u_{r} \geq 0 ; r=1, \ldots, s \\
& v_{i} \geq 0 ; i=1, \ldots, m \\
& \tilde{u}_{0}: \text { free in sign }
\end{aligned}
$$

Akin to the CCR model, the fractional BCC model can be transformed into a less computationally intensive formulation consisting of a primal and dual form.

\section{Primal BCC Input-Oriented Model (3.15)}

Maximize: $\quad w_{0}=\sum_{r=1}^{s} u_{r} y_{r 0}-\tilde{u}_{o}$

Subject to $\quad \sum_{r=1}^{s} u_{r} y_{r j}-\sum_{i=1}^{m} v_{i} x_{i j}-\tilde{u}_{o} \leq$ 0

$$
\begin{aligned}
& \sum_{i=1}^{m} v_{i} x_{i 0}=1 \\
& -u_{r} \leq-\varepsilon \\
& -v_{i} \leq-\varepsilon \\
& \tilde{u}_{0}: \text { free in sign }
\end{aligned}
$$

Dual BCC Input-Oriented Model (3.16)

Minimize: $\quad z_{0}=\theta-\varepsilon\left[\sum_{i=1}^{m} s_{i}^{-}+\sum_{r=1}^{s} s_{r}^{+}\right]$

Aside from the addition of the $\tilde{u}_{o}$ variable, the primal BCC form functions in the same fashion as the primal CCR form. The sole difference between the CRS dual form and the BCC dual form is that the $\lambda_{j}$ terms are forced to sum to one. This restriction removes the CCR model requirement that all DMUs are scale efficient. Moreover, this constraint reduces the feasible region for the linear program from a convex cone defined by the DMUs to a convex hull covering all the DMUs. This, in turn, increases the number of efficient DMUs. [CHAR94] Aside from this, the dual BCC and dual CCR formulations function in the same way. 
As with the CCR model, the DMUs in the BCC model are only efficient if $\theta$ is equal to one and the slacks $\left(\mathrm{s}_{\mathrm{r}}{ }^{+}, \mathrm{s}_{\mathrm{i}}{ }^{-}\right)$are equal to zero. The target projection to point $\left(\hat{x}_{0}, \hat{y}_{0}\right)$ on the efficient frontier can be obtained through the following formulation:

$$
\begin{array}{ll}
\hat{x}_{i 0}=\theta_{V R S}^{*} x_{i 0}-s_{i}^{-*} & (\mathrm{i}=1, \ldots, \mathrm{m}) \\
\hat{y}_{r 0}=y_{r 0}+s_{r}^{+*} & (\mathrm{r}=1, \ldots, \mathrm{s})
\end{array}
$$

\subsubsection{OUTPUT ORIENTED VRS MODEL}

The primal and dual formulations for the output-oriented BCC model are as follows:

Primal BCC Output-Oriented Model (3.19)

Minimize: $\quad \sum_{i=1}^{m} p_{i} x_{i 0}-\tilde{v}_{o}$

$$
\begin{aligned}
& \text { Subject to } \quad \sum_{r=1}^{s} q_{r} y_{r j}-\sum_{i=1}^{m} p_{i} x_{i j} \leq 0 \\
& \qquad \begin{array}{c}
\sum_{r=1}^{s} q_{r} y_{r 0}=1 \\
-p_{i} \leq-\varepsilon \\
-q_{r} \leq-\varepsilon \\
\tilde{v}_{0}: \text { free in sign }
\end{array}
\end{aligned}
$$

Dual BCC Output-Oriented Model (3.20)

Minimize: $\quad \eta-\varepsilon\left[\sum_{i=1}^{m} t_{i}^{-}+\sum_{r=1}^{s} t_{r}^{+}\right]$

$$
\begin{array}{ll}
\text { Subject to } & x_{i 0}=\sum_{j=1}^{n} x_{i j} \mu_{j}+t_{i}^{-} \\
& 0=\eta y_{r 0}-\sum_{j=1}^{n} y_{r j} \mu_{j}+t_{r}^{+} \\
1 & =\sum \mu_{j} \\
& 0 \leq \mu_{j}, \text { for } \mathrm{j}=1, \ldots, \mathrm{n} \\
& 0 \leq t_{i}^{-}, \text {for } \mathrm{i}=1, \ldots, \mathrm{m} \\
& 0 \leq t_{r}^{+}, \text {for } \mathrm{r}=1, \ldots, \mathrm{s}
\end{array}
$$

Similar to the input-oriented model, the output-oriented BCC model varies slightly from the CCR model.

Lastly, a DMU is VRS efficient if and only if $\eta$ is equal to 1 and the slacks $\left(\mathrm{t}_{\mathrm{r}}{ }^{+}, \mathrm{t}_{\mathrm{i}}{ }^{-}\right)$are equal to zero. Inefficient DMUs can target set using the following projection:

$$
\begin{array}{ll}
\hat{x}_{i 0}=x_{i 0}-t_{i}^{-*} & (\mathrm{i}=1, \ldots, \mathrm{m}) \\
\hat{y}_{r 0}=\eta_{v r s}^{*} y_{r 0}+t_{r}^{+*} & (\mathrm{r}=1, \ldots, \mathrm{s})
\end{array}
$$

\subsection{Additional DEA Models}

Aside from input-oriented and output oriented models, DEA offers a range of nonoriented models. These models provide the capability of simultaneously maximizing outputs 
while minimizing inputs. Non-oriented models have the same production possibility set as the aforementioned CCR and BCC models; however, they treat slacks directly in the objective function. [COOP07] The following sections provide an introduction to select non-oriented models; namely the Additive Model, Slacks Based Model and Multiplicative Model.

\subsubsection{ADDITIVE MODEL}

Based on the BCC model, the additive model was introduced by Charnes et al. in 1985. [CHAR85] As previously stated, the additive model is a non-oriented model that measures efficiency based on the slacks alone. The radial $(\theta)$ measure of efficiency is removed and the objective function is made to maximize the sum of slacks:

\section{Primal Additive Model (3.23)}

Maximize: $\quad z_{0}=\varepsilon\left[\sum_{i=1}^{m} s_{i}^{-}+\sum_{r=1}^{s} s_{r}^{+}\right]$

$$
\begin{array}{ll}
\text { Subject to } & y_{r 0}=\sum_{j=1}^{n} y_{r j} \lambda_{j}-s_{r}^{+} \\
& 0=x_{i 0}-\sum_{j=1}^{n} x_{i j} \lambda_{j}-s_{r}^{+} \\
1 & =\sum \lambda_{j} \\
& 0 \leq \lambda_{j}, \text { for } \mathrm{j}=1, \ldots, \mathrm{n} \\
& 0 \leq s_{i}^{-}, \text {for } \mathrm{i}=1, \ldots, \mathrm{m} \\
& 0 \leq s_{r}^{+}, \text {for } \mathrm{r}=1, \ldots, \mathrm{s}
\end{array}
$$

\section{Dual Additive Model (3.24)}

Minimize: $w_{0}=\sum_{i=1}^{m} v_{i} x_{i 0}+\sum_{r=1}^{s} u_{r} y_{r 0}+\tilde{u}_{0}$

Subject to

$$
\begin{aligned}
& \sum_{r=1}^{s} u_{r} y_{r j}-\sum_{i=1}^{m} v_{i} x_{i j}-\tilde{u}_{0} \leq 0 \\
& -u_{r} \leq-\varepsilon \\
& -v_{i} \leq-\varepsilon
\end{aligned}
$$

Under the additive model, a unit is only efficient if both slacks are zero. If a unit is deemed inefficient, its efficiency score is based on the combined input excess and output shortfall that will project the unit onto the frontier with non-negative slacks. Although the sources and amounts of a unit's efficiency differ between the additive and BCC model, an efficient DMU under the BCC model will also be efficient under the additive model and vice versa. Additionally, the shape of the efficient frontier remains the same as the VRS BCC model. This is due to the inclusion of the convexity constraint $\left(1=\sum \lambda_{j}\right)$ in the primal model and the $\tilde{u}_{0}$ variable in the dual model. Moreover, the additive model is translation invariant in both the inputs and outputs meaning the translation of the original input or output values will result in the same optimal solution. [COOP07] 


\subsubsection{SLACKS BASED MODEL (SBM)}

The Slacks Based Model (SBM) is an extension of the Additive model which allows the efficiency evaluation to be unit invariant. This means that the model's efficiency measure is invariant with respect to the unit of measurement of the input and output variables. Furthermore, the efficiency measure is monotone decreasing in the slacks of the input and output variables. [COOP07] The general formulation for the SBM model is as follows:

$$
\text { Minimize } \quad \rho=\frac{1-\frac{1}{m} \sum_{i=1}^{m} \frac{s_{i}^{-}}{x_{i 0}}}{1+\frac{1}{s} \sum_{r=1}^{s} \frac{s_{r}^{\mp}}{y_{r 0}}}
$$

Subject to

$$
\begin{array}{ll}
\sum_{j=1}^{n} x_{i j} \lambda_{j}+s_{i}^{-}=x_{i o} & (\mathrm{i}=1,2, \ldots, \mathrm{m}) \\
\sum_{j=1}^{n} y_{r j} \lambda_{j}+s_{r}^{-}=y_{r o} & (\mathrm{r}=1,2, \ldots, \mathrm{m}) \\
\mathrm{j}=1,2, \ldots, \mathrm{n} & \\
\lambda_{j}, s_{i}^{-}, s_{r}^{+} \geq 0 &
\end{array}
$$

Where $\quad 0 \leq \rho \leq 1$

Under SBM, a DMU is efficient if $\rho^{*}=1$ or in other words the slacks are zero. As with the additive model, the inefficient DMUs can target set using a projection of the combined input excess and output shortfall onto the frontier. This is denoted as:

$$
\begin{array}{ll}
\hat{x}_{0}=x_{i 0}-s_{i}^{-*} & (\mathrm{i}=1,2, \ldots, \mathrm{m}) \\
\hat{y}_{0}=y_{r 0}-s_{r}^{+*} & (\mathrm{r}=1,2, \ldots, \mathrm{m})
\end{array}
$$

\subsubsection{Multiplicative Model}

The multiplicative model was developed by Charnes et al. in 1982. [CHAR82] Unlike the previously introduced DEA models, the Multiplicative Model uses multiplicative combinations for the inputs and outputs yielding a piece-wise log-linear (Cobb-Douglas type) efficient frontier. In layman's terms, the multiplicative model is obtained by taking the anti-logs of the additive model, thus changing the summation signs $\left(\sum\right)$ to product signs $\left(\prod\right)$. The multiplicative model does not have a radial proportional variable and identifies inefficiencies through slack values alone. In 1983, this model was extended further, giving it non-dimensional properties. [CHAR83] 
Although not extensively used, the multiplicative model offers a few opportunities to extend the range of applications for DEA. Firstly, multiplicative models do not confine efficient frontiers to concavity, but allow them to exist with both concave and non-concave regions. Furthermore, these models can provide exact estimates of elasticities. [BANK04] For a more detailed description of the multiplicative model, the reader is encouraged to consult [COOP07].

\subsection{RESULTS FROM DEA}

DEA can provide several important pieces of information that can give further insight into the efficiency and performance characteristics of a DMU. The following sections discuss some key results obtained from DEA, including technical, scale and mix efficiencies, peer groups and target setting and the returns to scale of the operating environment.

\subsubsection{TECHNICAL AND SCALE EFFICIENCY}

As previously discussed, the original CCR DEA model made the assumption of constant returns to scale and excluded the effects of scale size on efficiency. The CCR model measures a unit's overall technical efficiency which is an aggregation of technical efficiency and the effects of scale size. Therefore, any inefficiency calculated in the CCR model could be a result of inefficient technologies, non-optimal scale size or a combination of both. To separate the overall efficiency into its components, the BCC model must be employed using the same data set. The BCC model imposes less strict assumptions of variable returns to scale and results in a measure of technical efficiency alone. Subsequently, taking the ratio of CRS efficiency over VRS efficiency will give the unit's scale efficiency, which measures each DMU's distance from its optimal scale size.

Figure 3.2 provides a comparison between the CCR and BCC DEA models, with CCR represented by a dashed straight line and BCC by a solid curve. This graph can be used to ascertain information about a DMUs technical and scale efficiencies. Aside from being the only CRS efficient producer, DMU G is located on both efficient frontiers, and exhibits the highest average productivity for the given inputs. DMU G is therefore said to be operating at its most productive scale size (MPSS). This term was coined by Banker [BANK84] and refers to a DMU that is operating at the highest output per unit of input and that has the highest slope. For a more detailed look into MPSS, the reader is encouraged to review Cooper et al.. [COOP07]

Referring back to Figure 3.2, it is noted that the BCC frontier is comprised of DMUs F, $\mathrm{G}, \mathrm{H}$ and I. All of these units are therefore considered technically efficient, but only those that 
also lie on the CCR frontier can be considered scale efficient. It follows that DMU G is the only unit that is both technically and scale efficient within this set of DMUs.

\subsubsection{EFFICIENCY}

Both technical and scale efficiency are radial measures and thus do not include any nonradial inefficiencies such as the slacks based measure (SBM) of efficiency. Mix inefficiencies are calculated by dividing the SBM efficiency score $(\rho)$ by the CCR efficiency score $(\theta)$. Mix efficiency will have a value between zero and one, with one denoting that the optimal CCR solutions have no slacks and that the model is not subject to any mix inefficiencies.

\subsubsection{Peer Groups ANd TARget Setting}

DEA models also provide target setting opportunities and peer group evaluations. For each inefficient DMU, DEA provides a reference group composed of efficient peers. The target projections for each DMU will vary based on the model used, the shape of the frontier and the orientation selected. It follows that some inefficient DMUs will have multiple peers and others will only have one. The peer groups for inefficient DMUs are determined by the values given to the $\lambda$ variables. These variables represent the proportion of each efficient DMU that an inefficient DMU should be imitating.

\subsubsection{RETURNS TO SCALE}

As previously discussed in Sections 3.3 and 3.4, there exist both constant returns- to-scale and variable returns-to-scale DEA models. The CCR model's frontier has a constant slope meaning that any increase in inputs will result in a proportional increase in outputs. On the other hand, the slope of the BCC model's frontier is allowed to change resulting in increasing, decreasing or constant returns to scale (IRS, DRS and CRS respectively). IRS occurs when marginal productivity is greater than average productivity; consequently an increase in input produces a proportionally larger increase in outputs. Conversely, DRS occurs when average productivity is greater than marginal productivity.

Based on the $\tilde{u}_{0}$ variable, the BCC model can establish whether a unit is operating under IRS, DRS or CRS. Moreover, under certain circumstances it is possible for the $\tilde{u}_{0}$ variable to have multiple solutions. To address this issue, Sueyoshi developed a method of identifying whether multiple solutions existed and established two unique DEA formulations. [SUEY99] 
Additionally, the convexity constraint $\left(1=\sum \lambda_{j}\right)$ in the BCC model can be altered to limit the frontier to specific returns to scale assumptions. If it is assumed that the scale of a DMU cannot be reduced but only increased, the convexity constraint becomes $1 \leq \sum \lambda_{j}$ resulting in a non-decreasing returns-to-scale model (NDRS). In an NDRS model, the frontier will only show increasing or constant returns-to-scale. Similarly, a non-increasing returns-to-scale (NIRS) model can be obtained by changing the convexity constraint to $1 \geq \sum \lambda_{j}$ resulting in only constant or decreasing returns-to-scale. A third model, known as the generalized returns-to-scale (GRS) model involves setting a lower and upper bound for the convexity constraint.

\subsection{EXTENSIONS To BASIC DEA ModELS}

In order to improve model accuracy and provide a more realistic representation of the DMUs under consideration several extensions and modifications to the aforementioned DEA models are now available. From the available extensions, a select few, namely non-discretionary variables, categorical variables and restricted multiplier models, have been employed in this study to broaden the range of compatible data types and to provide a means of restricting multipliers within realistically feasible limits. These extensions are discussed in detail in the following sections.

\subsubsection{NON-DISCRETIONARY VARIABLES}

In order to accurately represent a DMU's production process, all pertinent variables affecting this process should be included in the model. However, there are cases when one or more pertinent variables are not controllable by management; thus including them in the model in the same manner as the controllable variables could produce nonsensical results. For example, the surrounding geographical environment of a bank branch is not something that management can control; nonetheless, these factors can have a great impact on the productivity, performance and services of the branch and therefore should be included in the study.

These uncontrollable variables, known as non-discretionary variables, can be dealt with in a relatively simple manner. Non-discretionary variables should be removed from the objective function of the linear program but included in the constraints. This assures that their presence is still accounted for and that their values remain constant while only the discretionary variables are optimized. For additional information about the inclusion and treatment of non-discretionary variables, the reader is encouraged to consult Cooper et al.. [COOP07] 


\subsubsection{CATEGORICAL VARIABLES}

There are yet other situations when a pertinent variable has a discrete value from a set of two or more discrete values. Introduced by Banker and Morey [BANK86], these variables are referred to as categorical variables and, amongst other things, can include the presence of ATM units, weekend opening availability of branches and categorical representation of branches (i.e. rural, urban, metropolitan). There are two classifications of categorical variables, controllable and uncontrollable, which must be dealt with in different manners.

Controllable categorical variables refer to those which management have decided upon. For example, management may decide to rate bank branches as poor, average and good based on their service hours. In order to fairly assess branches based on these categorical variables, they are only compared against branches that fall in the same or worse performing categories. (i.e. average branches would only be compared to average and poor branches) This is achieved by implementing a linear programming algorithm. This algorithm also allows the DEA model to produce a unique frontier for each hierarchical level providing the ability to evaluate each DMU against different frontiers. Additionally, the recommended reference set is restricted to DMUs which exist within the same hierarchal level.

As with controllable categorical variables, non-controllable categorical variables can be handled using hierarchies. A similar linear programming algorithm can be used to ensure that inefficient DMUs are only compared to efficient DMUs operating in the same or worse environments. Again, this would produce separate frontiers and would restrict the reference set of each DMU to those within its hierarchical level.

It should be noted that these methods should not be used for non-comparable categories. Instead, either a separate analysis should be performed, or cultural adjustment factors should be used. The use of cultural factors is discussed in detail in [YANG02]. For additional insight into categorical variables refer to Cooper et al.. [COOP07]

\subsubsection{Restricted MUltiplier MOdels}

DEA does not require a priori knowledge about the input and output variables but assigns multipliers to each DMU's variables such that the DMU looks as efficient as possible. However, there are situations where the assigned multipliers do not properly represent what is realistically feasible. In these cases, the use of additional information to impose informed multiplier restrictions can result in more accurate efficiency estimates and more realistic depictions of relative efficiency and best performers. These multiplier restrictions can be based on managerial 
and organizational factors, physical or production limitations or any other constraint affecting the feasible range of inputs and outputs.

The concept of multiplier restriction was first introduced by Dyson et al. [DYSO88] who imposed upper and lower bounds on each multiplier. Subsequently in 1989, Charnes et al. [CHAR89] established the Cone-Ratio Method which restricted the feasible region of various multipliers to polyhedral convex cones that were defined by non-negative direction vectors. Additionally, Thompson et al. [THOM90] introduced the Assurance Region Method which imposed constraints in terms of absolute numbers or in terms of ratios assigned to the multipliers. These constraints are represented mathematically as:
Absolute Numbers (3.28)
$L_{i} \leq v_{i} \leq U_{i}$
Where: $\quad \mathrm{L}_{\mathrm{i}}$ is the upper bound
$\mathrm{U}_{\mathrm{i}}$ is the lower bound

Ratio Constraints (3.29)

$L_{1,2} \leq \frac{v_{2}}{v_{1}} \leq U_{1,2}$

Using the absolute number form of the Assurance Region Method is only possible when actual prices or levels of prices are known. Alternatively, the ratio constraint form can be used when the specific values are unknown but a general range of values is known.

If properly employed, restricted multiplier models can be very useful in providing more accurate and realistic modeling and can help avoid zero multipliers. It should be noted that, in general, these restrictions produce lower efficiency scores as the model is no longer able to choose the most advantageous multiplier. For additional information and detailed descriptions of the aforementioned restricted multiplier methods the reader is encouraged to consult Cooper et al.. [COOP07]

\subsection{STRENGTHS AND LiMitATIONS OF DEA}

DEA offers several advantages over other commonly used performance measures, and, as with all analysis methods, has some intrinsic weaknesses. The following sections provide a synopsis of these strengths and limitations.

\subsubsection{STRENGTHS}

1. Ability to handle multiple inputs and outputs: Given a sufficient number of degrees of freedom; DEA models can handle any number of inputs and outputs. Other approaches, including regression analysis and SFA, can handle only multiple inputs or multiple outputs, but not both. (i.e. one input and multiple outputs or vice versa) 
2. Does not require a priori specification of functional form: Unlike parametric analysis techniques, DEA does not require any prior knowledge of the relationships between the inputs and outputs.

3. Does not require consistent metrics: A DEA model is able to simultaneously handle different units without any effect.

4. Does not require prior knowledge of variable multipliers: The DEA model assigns variable multiplier and thus does not require them to be pre-specified. Moreover, multiplier ratios and range constraints can be applied to a model to provide more accurate and realistic results.

5. Provide target setting capabilities: DEA compares DMUs with a peer or combination of peers to obtain the DMU`s efficiency score. Should a DMU be inefficient, it can be projected onto the efficient frontier to provide realistic target setting.

6. Provides a single performance score: DEA produces a simple comprehensive efficiency score that characterizes a DMU`s relative performance.

\subsubsection{LIMITATIONS}

1. Does not account for random error: DEA does not account for measurement error or other noise but assumes that all deviations from the frontier are caused by inefficiencies. If random error is present, DEA may produce inaccurate results.

2. Unable to accurately model small sample sizes: When the degrees of freedom of a DEA model are too low (i.e. too few DMUs and too many variables), the model will tend to produce higher than normal average efficiency scores with many units appearing on the frontier.

3. Provides a relative efficiency score: DEA provides relative efficiency scores that are based on the specific DMUs studied. Should an important or highly efficient DMU be excluded from the analysis, the scores provided will not be as accurate.

4. Outliers can heavily influence results: Outliers operating with unfair advantages can greatly skew resulting efficiency scores. Other DMUs will experience lower overall efficiency scores which, in reality, are not significant.

5. It is retrospective: DEA is a rear-facing performance measure that does not allow for future projections. 


\section{Chapter 4: DEA Modeling}

This chapter presents an introduction to the Canadian banking system, as well as an overview of the "Bank". A discussion of the variable selection and DMU characterization techniques is then provided. Subsequently, each model, from objective to results, is discussed in its own respective section. A thorough discussion and analysis of the DEA results are provided in Chapter 5.

\subsection{Canadian Banking System}

The banking system in Canada is regulated by the Canadian Bank Act (S.C. 1991, c.46), last revised in 1991 by the Office of the Superintendent of Financial Institutions (OSFI). [DJC11] Established in 1987 under the 'Office of the Superintendent of Financial Institutions Act', OSFI is a federal agency that regulates and supervises federal financial institutions and private pension plans. OSFI is responsible for assuring that institutions are working in sound financial conditions and are complying with their governing laws. Additionally, OSFI contributes to the development and administration of regulations and legislations. [OSFI11]

Currently, OSFI regulates 77 banks; 23 are domestic, 26 are foreign bank subsidiaries and 23 are full-service foreign banks. Banks in Canada are further categorized into Schedule I, II and III. Schedule II and III respectively deal with foreign bank subsidiaries and the bank branches of foreign institutions. Schedule I encompasses all of the domestic banks. [CBA10] In total, these institutions manage nearly $\$ 3.7$ trillion in assets. [OSFI11]

Canadian Banks, as well as certain Schedule II banks, are eligible for deposit insurance provided by the Canadian Deposit Insurance Corporation (CDIC), a federal crown corporation. Created by Parliament in 1967 , the CDIC insures deposits up to $\$ 100,000$ per depositor in each member institution and reimburses depositors for the amount of any insured deposits should any member fail. [CDIC12] The CDIC helps to maintain the stability of the Canadian financial system.

As of October $31^{\text {st }} 2011$, Canada's domestic banks held approximately $\$ 3.1$ trillion with the top five banks, referred to as the "Big Five", accounting for nearly 92\% of this value. [CBA10] The Big Five, which include Bank of Montreal (BMO), Bank of Nova Scotia (BNS), Canadian Imperial Bank of Commerce (CIBC), RBC Financial Group (RBC) and TD Canada 
Trust (TD), operate in what some call is an oligopoly. Small domestic and foreign banks and new entrants are as yet little competition for these market dominant institutions; however, there is substantial competition amongst themselves. The Big Five's shares are widely held, with any entity allowed to hold a maximum of twenty percent. [DJC11] In the last quarter of 2011, the "Big Five" alone held $\$ 1.5$ trillion in total loans, 2 trillion in deposits, and $\$ 168$ billion in total shareholders' equity. [CBA10]

Canadian banks service a wide range of customers including individuals, small and medium-sized businesses, larger corporations, and governments. They offer a variety of banking, investment and financial services through large branch networks. Canada is considered to be one of the most heavily "branched" countries in the industrialized world, with more than 6,150 full service branches. Moreover, Canadian banks employ in excess of 267,200 people and provide the service of over 17,000 Automated Banking Machines (ABMs). [CBA10]

\section{2 “BANK" OVERVIEW}

One of the "Big Five" Canadian banks, the Bank evaluated in this study ranks within the top 75 banks worldwide in terms of asset size. [CBA10] The Bank includes a branch network of more than 1,000 branches and employs over 40,000 branch and corporate personnel. The Bank practices retail, commercial and corporate banking and offers an extensive range of financial products and services. The following table (Table 4.1) provides a partial list of products and services offered by the Bank. These services are administered through several channels including in-branch, debit cards, Automated Banking Machines (ABMs), and internet and telephone banking.

\section{Table 4.1: Bank’s Retail and Commercial Products and Services}

Retail and Commercial Products and Services

- Bank Accounts (Chequing, Savings)

- Lines of Credit

- Mortgages and Other Loans

- Mutual Funds and Investment Services
- Investment Banking and Brokerage

- Credit Card

- Foreign Exchange, Wires and Bank Drafts

- Insurance Brokerage 


\subsection{DATA OVERVIEW AND TREATMENT}

The Bank provided a substantial data set, with upwards of 70 variables, including categorical (region, market etc.) and non-categorical (products, assets etc.) variables for over 1000 of its branches. This considerable data set provided the opportunity to evaluate the problem at hand from several vantage points and to construct multiple unique models. Although issues with dimensionality were unlikely with such a large number of DMUs, DEA loses discriminatory power as the dimensionality of the production space increases. Therefore, to obtain more informative and distinct, results careful variable selection was performed by means of correlation analysis.

As DEA uses peer references and produces relative efficiency scores, the presence of outliers can greatly impact the validity of the obtained results. It follows that outliers must be identified and removed from the data set. This was performed through the application of two methodologies, namely the stripping of the efficient frontier and statistical data analysis.

For comparison purposes, the data was also segmented by select variables. Categorical variables were segmented by their individual categories while non-categorical variables were segmented using k-means segmentation. This process, along with the aforementioned data treatments are discussed in the following sections.

\subsubsection{VARIABLE SELECTION}

When selecting variables for inclusion in a specific model, it is important to keep the model's objective in mind as well as avoid redundancy in the variables. Reducing the number of highly correlated variables (proxies) helps increase the discriminatory power of DEA without adversely affecting the final efficiency scores. In this study, correlation analysis was used to aid with variable selection. Discretion was also used in the removal of variables, as, from a managerial standpoint, it may make more sense to leave certain variables in the model even if they are highly correlated.

Correlation analysis is a very commonly used technique for DEA variable selection. It employs the use of the correlation coefficient $(\rho)$ which measures how two variables vary together. The coefficient's value can range from +1 , denoting a perfect positive linear relationship, to -1 , denoting a perfect negative linear relationship, with 0 representing no correlation at all. The correlation coefficient can be calculated using the following formula:

$$
\rho_{v 1, v 2}=\frac{\operatorname{cov}\left(v_{1}, v_{2}\right)}{\sigma_{v 1} \sigma_{v 2}}=\frac{E\left[\left(v_{1}-\mu_{v 1}\right)\left(v_{2}-\mu_{v 2}\right)\right]}{\sigma_{v 1} \sigma_{v 2}}
$$


Where: $\quad v_{1}, v_{2}: \quad$ are the variables being compared

$\operatorname{cov}\left(v_{1}, v_{2}\right):$ is the covariance of $v_{1}$ and $v_{2}$

$\sigma: \quad$ represents the standard deviation of the specified variable

$\mathrm{E}: \quad$ is the expected value operator

$\mu$ : the mean of the specified variable

Correlation analysis, in terms of variable selection, requires that the correlation coefficient be calculated for every combination of inputs and outputs. When there are two inputs (or outputs) that are highly correlated with one another $(|\rho|>0.95)$, this suggests that the variables are too similar and convey essentially the same information. It follows that one of the two should be removed, or the two should be combined together. On the other hand, when comparing an input variable with an output variable, there should be some degree of correlation $(>0.50)$ between the two, ensuring that they are both statistically significant.

\subsubsection{DMU CHARACTERISATION}

The results of a DEA model can be heavily skewed by the existence of outliers, which can include DMUs with erroneous data, and non-comparable DMUs (i.e. oil and gas branches, and real estate branches). To ensure the credibility of the model results, it is essential that the data be cleaned of outliers. For this study, the primary means of outlier removal was stripping the efficient frontier. When this method was found unsuitable (i.e. no/small changes in mean efficiency between strips), statistical data analysis was performed.

\subsubsection{STRIPPING OF THE EFFICIENT FrONTIER}

DEA provides relative efficiency measures and peer references that are based on the efficient units that compose the efficient frontier. It follows that the outliers that find themselves on the frontier can affect the efficiencies of numerous units. Consequently, these efficient outliers pose the greatest risk to the soundness of the results. Although it is generally found that branch related DEA models return between 25-40 percent efficient units, the models employed in this study take a different approach, and their results are not necessarily comparable to those of traditional (typically production) models. Thus, to assess the effectiveness of each strip, the difference in average efficiency score and number of efficient units was examined. It should be noted that not all of the units stripped from the frontier are necessarily outliers, and each should be carefully considered by management. The implementation of this technique, differing for each 
model, is discussed in each model's respective section, while the efficacy of this methodology is examined in Chapter 5.

\subsubsection{StATISTICAL DATA ANALYSIS}

When stripping was found to be inappropriate, statistical data analysis was used to identify outliers. To begin, the coefficient of variation was calculated for each variable by dividing the standard deviation by the mean. The coefficient of variation is a normalized measure of the dispersion of a variable with respect to the mean of its population. Higher coefficients of variation indicate that there is greater variation in the variable values and thus the distribution histogram of the variable with the largest coefficient of variation was examined. Consequently, outliers and extreme valued units were removed. The application and results from this methodology is discussed in detail in Section 4.8.

\subsubsection{SEGMENTATION}

Segmenting the results of a DEA model is another commonly used analysis tool that can provide further insight into the meaning and significance of the results. In this study, both categorical and non-categorical segmentations were used once the DEA models had been run. The categorical segmentations were by Market (Alberta, Atlantic Canada, GTA-Central, GTAEast, GTA-West, Northern Ontario, Prairies, Quebec, Southern Ontario, Vancouver Island \& Northern Territories and Vancouver Lower Mainland) and by Urban/Rural, both of which were defined by the Bank.

DMUs were also segmented by "funds managed" and by "existing customer base". As these are non-categorical variables, k-means segmentation, one of the most popular cluster analysis methods, was used on the original set of 1041 DMUs. K-means segmentation groups a DMU with 'like' DMUs by partitioning n observations (1041 DMUs) into k clusters (4 clusters) in which each observation belongs to the cluster with the nearest mean. This methodology can be used for single- or multi- variable segmentations.

For ease and continuity, the "funds managed" segments are referred to as follows:

Table 4.2: Funds Managed Segments

\begin{tabular}{|l|c|c|}
\hline \multicolumn{1}{|c|}{ Notation } & Segment Range (\$) & \# of DMUs \\
\hline Funds-S & $3,313,956.60-80,282,296.80$ & 524 \\
\hline Funds-M & $80,481,614.72-170,379,805.97$ & 387 \\
\hline Funds-L & $171,200,464.57-336,017,752.75$ & 115 \\
\hline Funds-XL & $359,066,886.41-1,165,724,792.51$ & 15 \\
\hline
\end{tabular}


Similarly for the "existing customer base" segments, the following notations are used throughout:

Table 4.3: Existing Customer Base Segments

\begin{tabular}{|l|c|c|}
\hline \multicolumn{1}{|c|}{ Notation } & Segment Range (Customers) & \# of DMUs \\
\hline Cust-S & $342-4,092$ & 409 \\
\hline Cust-M & $4,117-8,038$ & 399 \\
\hline Cust-L & $8,186-14,314$ & 186 \\
\hline Cust-XL & $14,524-37,198$ & 47 \\
\hline
\end{tabular}

\subsubsection{LOCAL ANALYSIS}

The significance and usefulness of the results obtained from a DEA analysis are highly dependent on whether or not the DMUs are, in fact, comparable. Although the DMUs used for this study are under the same regulations and corporate culture, there may be size or environmental factors that impact the operations of each branch. Therefore, to better assess the efficiency of the DMUs, and to provide more realistic peer references and target setting, a local analyses were performed. Local analyses offer the opportunity to compare units only to those that are most comparable. The DMUs are segmented based on a chosen characteristic or variable and then local DEA analyses are run for each segment, where only the DMUs in that segment are included. It follows that the increase in DEA scores resulting from local analysis can be attributed to the decrease in differentiation between compared DMUs.

As with the global analysis segmentation, the local analysis employed k-means segmentation. In order to account for the high volatility of certain variables and to better segment the DMUs, the two variables with the highest coefficients of variation were used to calculate the segments for each model. Local analyses were only used for the Delta model. Their application and results are discussed further in Section 4.8.

\subsubsection{SENSITIVITY ANALYSIS}

Sensitivity analysis can provide further insight into which variables are highly pertinent to the results of a DEA model and which affect the results less. For the purpose of this study, two forms of sensitivity analysis were applied to the "2010-2011 Period Growth" model. First, the efficiency contribution measure was used to determine the influence of each variable on the DEA scores. This was done by removing each variable one at a time and re-running the model with the remaining variables. The difference in mean scores between the re-run model and the original model were then examined. 
Subsequently, the Wilcoxon Rank-Sum test was used. This test is a non-parametric statistical hypothesis test that is used when comparing two related samples to assess whether their population mean ranks differ. It can be used when the population cannot be assumed to be normally distributed, making it an ideal way of determining whether or not two sets of DEA scores have significant differences. [COOP07]

Cooper et al. [COOP07] offer a simplified version of the test where the two independent data sets of efficiency scores are defined as $A=\{a 1, a 2, \ldots, a m)$ and $B=\{b 1, b 2, \ldots, b n\}$. The data from $\mathrm{A}$ and $\mathrm{B}$ are combined to form a set of $\mathrm{m}+\mathrm{n}$ observations in a new data set referred to as $\mathrm{D}$. This new data set is then ordered from largest to smallest and ranked, with any identical values in $D$ receiving a mid-rank (sum of ranks/number of identical values). The sum $(\mathrm{S})$ of the ranks of either data set $\mathrm{A}$ or $\mathrm{B}$ is taken and $\mathrm{S}$ is then normalised using the following equation:

$$
T=\frac{(S-m(m+n+1) / 2)}{\sqrt{(m n(m+n+1) / 12)}}
$$

According to Cooper et al., T has an approximately standard normal distribution. Using the value obtained for $\mathrm{T}$, the null hypothesis that the two groups have the same population at a level of significance of $\alpha$ can be checked. The hypothesis is rejected if either $\mathrm{T} \leq-\mathrm{T} \alpha / 2$ or $\mathrm{T} \geq \mathrm{T} \alpha / 2$.

Although this methodology is relatively simple when dealing with small data sets, the requirement of mid-ranks becomes increasingly difficult and time consuming for large datasets. For the purpose of this study, the Matlab function 'ranksum' was used, which employs the traditional Wilcoxon Rank-Sum Test. Like its simplified version, the null hypothesis of the Wilcoxon Rank-Sum Test is that the two groups have the same population. A significance level of $\alpha=0.05$ was used for this test and thus the hypothesis was rejected if either $Z \leq-1.96$ or $\mathrm{Z} \geq 1$.96. The $\rho$-statistic, also provided by the Matlab function, is a non-parametric measure of overlap between the two data sets and is widely used in studies of categorization. It can range in value from 0 to 1 with both extreme values representing the complete separation of the distributions and 0.5 representing complete overlap.

\subsection{STUDY FocUS}

Like any business or corporation, the Bank continually strives to improve their growth, margins and increase their market share in order to remain competitive. In the current economic times, the retention and growth of customers and funds has become increasingly difficult and is a major focus of the Bank. It was therefore decided that the focus of this study would be to 
examine the growth potential of the Bank's branches and identify the best performers. The models presented herein each offer a unique perspective and direction for attaining their shared goal.

An extensive data set was provided offering the opportunity to examine branch growth from several vantage points. In total, four model perspectives and six models, each using a unique combination of inputs and outputs, were defined. For each model, correlation analyses were performed followed by the execution of output-oriented DEA models. A detailed description of each model and their results are provided in Sections 4.5 through 4.8.

\subsection{0-2011 PERIOD GROWTH}

For select variables, both 2010 and 2011 yearend values were provided. This offered the unique opportunity of assessing the relative growth of the branch's business over a period of one year using the same input and output metrics. This viewpoint has not yet been used in literature and offers an exciting look into a new application of DEA. This Section introduces the "20102011 Period Growth" model and its objective, as well as discusses the variable selection and model building process. A synopsis of the obtained results is also provided.

\subsubsection{MODEL OBJECTIVE}

This model was to use 2010 yearend data for the input and 2011 yearend data for the output. In doing so, the model would assess each branch's 2010-2011 relative growth efficiency. To better define the objective of the model, variable selection was considered. The data set contained only 3 variables that were fully defined for both 2010 and 2011 yearend; funds managed, product use count and number of customers. To maintain model consistency, only these variables were considered, resulting in the following:

Table 4.4: 2010-2011 Period Growth Model

\begin{tabular}{|l|l|}
\hline \multicolumn{1}{|c|}{ Inputs (2010 Yearend) } & \multicolumn{1}{c|}{ Outputs (2011 Yearend) } \\
\hline Funds Managed (pfund1) & Funds Managed (pfund2) \\
Product Use Count (puc1) & Product Use Count (puc2) \\
Number of Customers (n_cust1) & Number of Customers (n_cust2) \\
\hline
\end{tabular}

'Funds Managed' is a metric used by the bank to denote the monetary sum of the products held and provided by the branch. It is comprised of total deposit balance, total RRSP and RRIF balances, total investment balance and total lending balance (loans made result in a 
positive lending balance). 'Product Use Count' (PUC) denotes the sum of the products held by clients of the branch. It is comprised of the number of deposit accounts, RRSP accounts, RRIF accounts, lending accounts and investment accounts. It is important to note that PUC does not consider multiples of the same account type held by one client. For example, whether a client held 1 or several deposit accounts, their PUC would still be 1. 'Number of Customers' is simply the number of customers who hold accounts at the branch.

The "2010-2011 Growth Period" model assesses the branch's ability to grow the number of customers and the amount of funds and products over the period of 2010-2011 given the number of customers, funds and products that existed at 2010 yearend. Branches whose 2010 production is high and whose 2011 production is low will perform worse than those with low 2010 production and high 2011 production values.

\subsubsection{Methodology}

Subsequent to the completion of the correlation analysis, an output-oriented CCR "20102011 Period Growth" model was run using all 1041 DMUs. For the second run, all of the efficient DMUs identified in the first run were removed and the model was re-run. This process was repeated for runs three and four. Once all four CCR runs were complete, the BCC model was run using all 1041 DMUs. A second BCC execution was performed using only those DMUs employed in the fourth run of the CCR model. This process is depicted in Figure 4.1.

Once each run of the model was executed and the data collected, the results were segmented by market, urban/rural, funds managed and existing customer base. Moreover, the returns to scale and mean scores were examined. To conclude, a sensitivity analysis was

performed using both the efficiency contribution technique and the Wilcoxon Rank-Sum test. 
CCR Model

$\underline{\text { BCC Model }}$
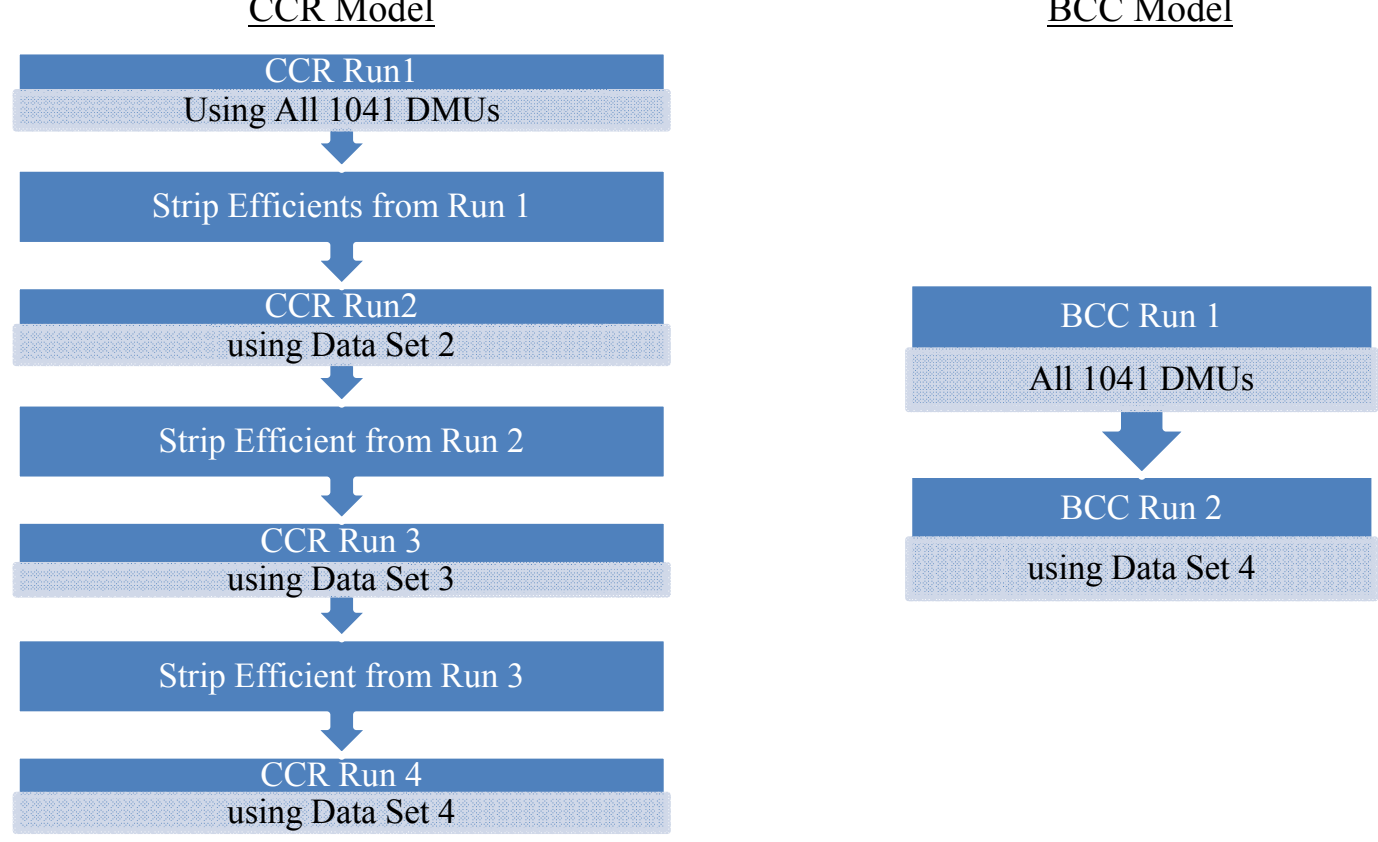

Figure 4.1: 2010-2011 Period Growth Model Execution Methodology

\subsubsection{CORRELATION ANALYSIS}

For managerial purposes, it was decided to keep all six inputs and outputs in the model; however, a correlation analysis was still performed and relevance indices (found in Table 4.4) were calculated.

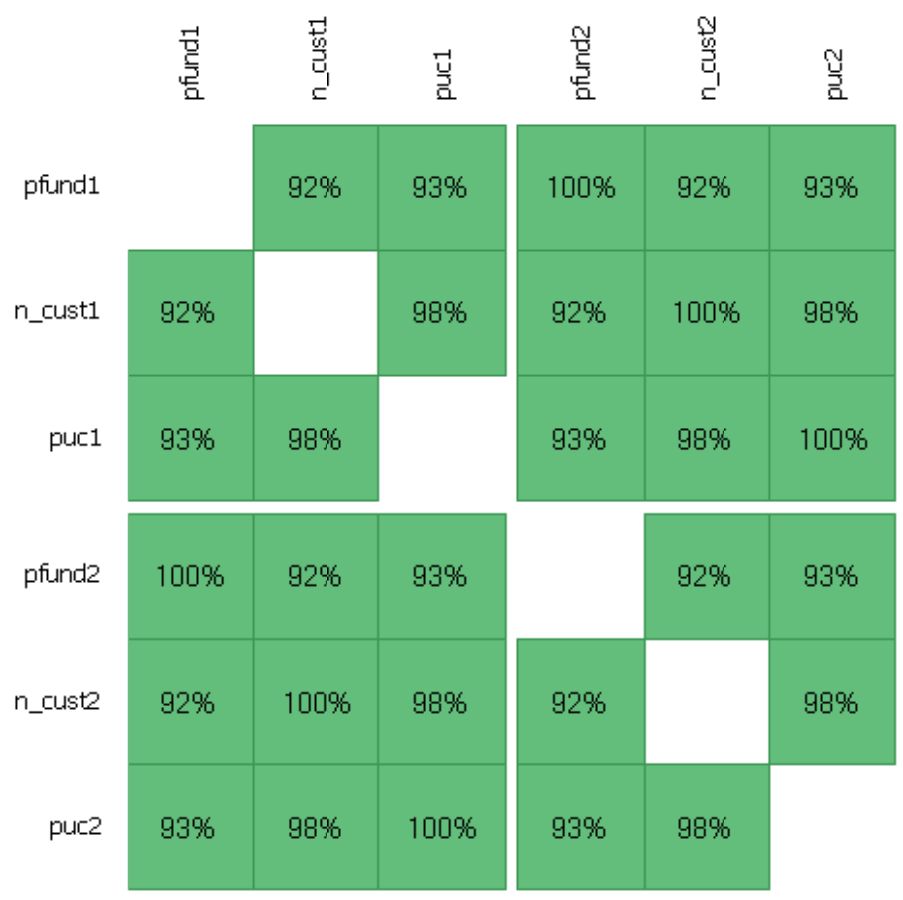

Figure 4.2: 2010-2011 Period Growth Model- Correlation Analysis 
The correlation analysis shows that these variables are highly correlated, as was expected. This suggests that one or more inputs and/or outputs could be removed from the model without losing any pertinent information. The high correlations between the inputs (outputs) show redundancy in the model; however, keeping the model as is provides a more complete picture of the changes realized over the year and offers better managerial guidance on how to improve performance.

\subsubsection{AVERAGE EFFICIENCY}

As described in the methodology, the CCR model was run four times with the efficient frontier stripped after each run. On the first run, the CCR model returned an average efficiency of 0.624 with a standard deviation of 0.09 and only 5 efficient units; an unusually low number. With the efficient units stripped and the CCR model re-run, an average efficiency of 0.775 with a standard deviation of 0.07 and 10 efficient units were observed. The third run returned an average efficiency of 0.823 with a standard deviation of 0.06 and 18 efficient units. The fourth and final CCR run produced an average efficiency of 0.89 with a standard deviation of 0.05 and 34 efficient units.

The first run of the BCC model returned a substantially higher average efficiency of 0.921 with a standard deviation of 0.05 . The BCC second run score was 0.943 with a standard deviation of 0.03. A summary of the model results is provided in Table 4.5. Efficiency distribution graphs for this and all other models are presented in Appendix 1.

Table 4.5: Summary of 2010-2011 Period Growth Model Average Efficiency Scores

\begin{tabular}{|c|c|c|c|c|c|c|}
\hline Run & \# of DMUs & $\begin{array}{c}\text { Average } \\
\text { Efficiency }\end{array}$ & Minimum & $\begin{array}{c}\text { \# of Efficient } \\
\text { DMUs }\end{array}$ & $\begin{array}{c}\text { \% of DMUs that } \\
\text { are Efficient }\end{array}$ & $\begin{array}{c}\text { Standard } \\
\text { Deviation }\end{array}$ \\
\hline \multicolumn{7}{|c|}{ CCR } \\
\hline 1 & 1041 & 0.624 & 0.476 & 5 & $0.48 \%$ & 0.089 \\
\hline 2 & 1036 & 0.775 & 0.624 & 10 & $0.97 \%$ & 0.069 \\
\hline 3 & 1026 & 0.823 & 0.717 & 18 & $1.75 \%$ & 0.061 \\
\hline 4 & 1008 & 0.892 & 0.789 & 34 & $3.37 \%$ & 0.047 \\
\hline \multicolumn{7}{|c|}{ BCC } \\
\hline 1 & 1041 & 0.921 & 0.640 & 40 & $3.84 \%$ & 0.048 \\
\hline 2 & 1008 & 0.943 & 0.821 & 63 & $6.25 \%$ & 0.031 \\
\hline
\end{tabular}

The low number of efficient DMUs obtained on the first run and the consistent increase in that number on subsequent runs tends to suggest that the resulting efficient frontiers were skewed by outliers and validates that the stripping technique was appropriate in this situation. Moreover, when comparing the CCR results to the BCC results, it is evident that the CCR model 
is much more susceptible to outliers dominating its frontier than is the BCC model - this completely agrees with theory.

\subsubsection{MARKET}

When split by market, the Prairies had the highest average fourth run CCR score of 0.910 and Northern Ontario had the highest average second run BCC score of 0.950. The West Greater Toronto Area had the lowest average fourth run CCR and second run BCC scores; 0.866 and 0.931, respectively. The standard deviations for the fourth run CCR model were all in the range of 0.4 , while the standard deviations of the $\mathrm{BCC}$ model were all in the range of 0.3 . A complete list of all average efficiency scores and standard deviations is provided for each model and segmentation in Appendix 2.

\subsubsection{URBAN/RURAL}

When split into urban and rural, the rural market had the highest average CCR score (0.906). For the BCC model, the urban and rural markets received the same average efficiency score of 0.943 and the same standard deviation of 0.03 .

\subsubsection{FUNDS MANAGED}

When split by funds managed, the Funds-S segment performed the best in the CCR model, with an average efficiency score of 0.901 and performed the worst in the BCC model with an average score of 0.935 . Funds-XL performed nearly as well as Funds-S in the CCR model with a score of 0.897 and performed the best in the BCC model with a score of 0.981 . The standard deviations varied between 0.02 and 0.05 .

\subsubsection{EXISTing Customer Base}

The results of the customer based segmentation were very similar to those observed when segmented by funds. The Cust-S segment performed the best in the CCR model with an average efficiency score of 0.912 and the worst in the BCC model with a score of 0.937. The Cust-XL segment performed the best in the BCC model with a score of 0.975 . The Cust-M and Cust-L segments performed very similarly in both models. Again, the standard deviations varied between 0.02 and 0.05 . 


\subsubsection{RETURNS TO SCALE}

Bank branch scale efficiency is calculated by taking the ratio of the CCR efficiency to the BCC efficiency. However, this can only be done when both models use the same set of DMUs. Therefore, for the purpose of this study, the scale efficiency was calculated for the models that contained all 1041 DMUs (first run CCR and BCC models) and for the fully stripped models (fourth run $\mathrm{CCR}$ and second run BCC). The scale efficiency of the first run was calculated to be $67.8 \%$, suggesting that non-optimal scale accounts for $32 \%$ of the overall inefficiency. Once fully stripped, the scale efficiency was calculated to be $94.5 \%$, a $26.6 \%$ improvement.

To determine whether the scale inefficiencies are due to increasing (IRS) or decreasing (DRS) returns to scale, an examination of the returns to scale (RTS) of each DMU was performed. The RTS of efficient units is based on the local slope of the frontier, while the RTS of inefficient units is based on the projection of the inefficient unit onto the efficient frontier.

For the BCC model's first run, $99.5 \%$ of the units were found to be decreasing returns to scale as opposed to $93 \%$ in the second. Of the remaining $0.5 \%, 0.1 \%$ was classified as CRS and $0.4 \%$ as IRS. For the second run, $0.3 \%$ was classified as CRS while $6.7 \%$ was classified as IRS. When split by funds managed, it was found that the majority of the CRS and IRS branches fell under the Funds-S segment. A summary of the RTS results can be found in Appendix 2.

\subsubsection{SENSITIVITY ANALYSIS}

\section{Efficiency Contribution Measure}

To determine the influence of each variable on the DEA scores of the DMUs, each variable was removed and the model was re-run with the remaining five variables. The difference in mean scores between the re-run model and the original model was then examined. The results are shown below in Tables 4.6 and 4.7. 
Table 4.6: Efficiency Contribution Measure- CCR Model

\begin{tabular}{|c|c|c|}
\hline Variable Removed & CCR DEA Score & \% of DMUs that are Efficient \\
\hline None & 0.62 & $0.48 \%$ \\
\hline \multicolumn{2}{|c|}{ Input } \\
\hline Funds Managed (2010) & 0.59 & $0.29 \%$ \\
\hline Product Use Count (2010) & 0.62 & $0.48 \%$ \\
\hline \# of Customers (2010) & 0.53 & $0.29 \%$ \\
\hline \multicolumn{2}{|c|}{ Output } \\
\hline Funds Managed (2011) & 0.59 & $0.48 \%$ \\
\hline Product Use Count (2011) & 0.53 & $0.29 \%$ \\
\hline \# of Customers (2011) & 0.62 & $0.48 \%$ \\
\hline
\end{tabular}

Table 4.7: Efficiency Contribution Measure- BCC Model

\begin{tabular}{|c|c|c|}
\hline Variable Removed & BCC DEA Score & \% of DMUs that are Efficient \\
\hline None & 0.92 & $3.84 \%$ \\
\hline \multicolumn{2}{|c|}{ Input } \\
\hline Funds Managed (2010) & 0.92 & $2.4 \%$ \\
\hline Product Use Count (2010) & 0.91 & $2.98 \%$ \\
\hline \# of Customers (2010) & 0.89 & $2.50 \%$ \\
\hline \multicolumn{2}{|c|}{ Output } \\
\hline Funds Managed (2011) & 0.92 & $3.07 \%$ \\
\hline Product Use Count (2011) & 0.88 & $2.40 \%$ \\
\hline
\end{tabular}

As is shown, the re-run scores only differ slightly in the CCR model and differ even less in the BCC model. Not surprisingly, in both models it appears that the input variable "number of customers' and the output variable 'product use count' had the largest impact on the DEA scores.

Wilcoxon Rank-Sum Test

The results of the Wilcoxon Rank-Sum test using the Matlab function 'ranksum' are provided below: 
Table 4.8: Wilcoxon Rank-Sum Test Results- CRR

\begin{tabular}{|c|c|c|c|c|}
\hline Variable Removed & $\rho$-Statistic & Z-value & Rank sum & Same Mean? \\
\hline \multicolumn{3}{|c|}{ Inputs (2010) } \\
\hline Funds Managed & $2.23 \mathrm{E}-27$ & 10.84 & 935528 & No \\
\hline Product Use Count & 0.7303 & 0.3447 & 1079473 & Yes \\
\hline \# of Customers & $6.31 \mathrm{E}-162$ & 27.1164 & $1.23 \mathrm{E}+04$ & No \\
\hline \multicolumn{5}{|c|}{ Outputs (2011) } \\
\hline Funds Managed & 0.1863 & 1.3217 & 1066074 & Yes \\
\hline Product Use Count & $3.35 \mathrm{E}-179$ & 28.5438 & $6.93 \mathrm{E}+05$ & No \\
\hline \# of Customers & 0.8262 & 0.2196 & 1081189 & Yes \\
\hline
\end{tabular}

Table 4.9: Wilcoxon Rank-Sum Test Results- BCC

\begin{tabular}{|c|c|c|c|c|}
\hline Variable Removed & $\rho$-Statistic & Z-value & Rank sum & Same Mean? \\
\hline \multicolumn{3}{|c|}{ Inputs (2010) } \\
\hline Funds Managed & 0.1733 & 1.3615 & $1.10 \mathrm{E}+06$ & Yes \\
\hline Product Use Count & $5.60 \mathrm{E}-05$ & 4.0289 & 1139459 & No \\
\hline \# of Customers & $7.67 \mathrm{E}-34$ & 12.1262 & $1.25 \mathrm{E}+06$ & No \\
\hline \multicolumn{5}{|c|}{ Outputs (2011) } \\
\hline Funds Managed & 0.0854 & 1.7201 & $1.11 \mathrm{E}+06$ & Yes \\
\hline Product Use Count & $2.15 \mathrm{E}-62$ & 16.6703 & $1.31 \mathrm{E}+06$ & No \\
\hline \# of Customers & 0.0052 & 2.7925 & 1045902 & No \\
\hline
\end{tabular}

The results of the Rank-Sum test are more informative than the results of the efficiency contribution analysis. The Rank-Sum test has identified several variables in both the CCR and BCC models that have a significant impact on the mean efficiency scores. In both models, the removal of the number of customers in 2010 and the product use count in 2011 resulted in a change in the mean, suggesting that these are statistically significant variables. However, the removal of funds managed in 2011 did not impact the mean in either the CCR or BCC models.

\subsection{Lost Accounts/Gained Accounts Model}

Amongst the datasets provided by the Bank were variables that described the churn of clients, and the gain and loss of accounts over the 2010 yearend to 2011 yearend period. When used in conjunction with DEA, these variables can provide insight into which branches are able to better retain customers and accounts and attract new customers and accounts. This section introduces the "Lost Accounts/Gained Accounts" model along with its objective. Subsequently, the variable selection and model building processes are discussed and a synopsis of the obtained results is provided. 


\subsubsection{MODEL OBJECTIVE}

Banks, along with many other service industries, take great interest in understanding the flux of clients, accounts and funds. The retention and development of existing clients and the acquisition of new clients each play a vital role in the market growth and overall health of a bank. Using variables that defined attrition, existing client growth and new client gain over the 2010-2011 period, the "Lost Accounts/Gained Accounts" model looks to assess which branches were best at retaining and growing their customer base, funds and products. A branch that retains customers, attracts new customers and grows the product use of existing customers will have a higher efficiency.

The model was constructed under the reasoning that a best performer should minimize attrition (inputs) and maximize growth and client gain (outputs). With this methodology in mind, the following model was developed:

Table 4.10: Lost Accounts/Gained Account Model

\begin{tabular}{|l|l|}
\hline \multicolumn{1}{|c|}{ Input } & \multicolumn{1}{c|}{ Output } \\
\hline Attrited PUC (Attr_puc1) & Existing Customers (Exist_n_cust) \\
Attrited Customers (Attr_n_cust) & New customers (New_n_cust) \\
Attrited Funds Managed (Attr_pfund1) & New PUC (New_puc2) \\
Existing Customer Growth in Managed & New Funds Managed (New_pfund2) \\
Funds (Exist_inc_pfund_input) & Existing Customer Growth in Managed \\
Existing Customer Growth in PUC & Funds(Exist_inc_pfund_output) \\
(Exist_inc_puc_input) & Existing Customer Growth in PUC \\
& (Exist_inc_puc_output) \\
\hline
\end{tabular}

In this model, the inputs consist of any variable that a branch wishes to minimize, in this case the attrition of clients, PUC and funds managed. Similarly, the outputs consist of those things that a branch wishes to maximize, namely retained customers, PUC and funds managed (referred to as existing customers/PUC/funds managed) and new customers, PUC and funds managed.

The model also considers the growth or decline in funds and products of the retained clients. For example, a retained client may have started the year with one deposit account, but decide to open a new account mid-year; ending the year with two accounts. This would be considered a positive growth. Conversely, a retained client may have started the year with two deposit accounts, but decide to close one of them mid-year; ending the year with only one account. This would be considered negative growth. Due to the fact that DEA does not handle 
negative numbers well, the original existing customer growth metrics for PUC and funds managed were each split into two variables; an input variable to represent negative growth and an output variable to represent positive growth. For clarification's sake, if a branch saw an overall increase in the PUC of existing customers, then the growth would be positive and the value would fall under the "existing customer growth in PUC" on the output side. A value of 0 would then be assigned to the "existing customer growth in PUC" on the input side. Had the branch seen a loss (negative growth), the absolute value of this loss would become an input, while 0 would be assigned to the output side.

\subsubsection{METHODOLOGY}

The Lost Accounts/Gained accounts model uses the same methodology as the "20102011 Period Growth" model, depicted in Figure 4.1. The CCR model was initially run with all 1041 DMUs. Subsequently the data set was stripped of all efficient DMUs and the model was run again (denoted as run 2). This process was continued for runs 3 and 4, resulting in a total of 3 frontier peels. The BCC model was also initially run with all 1041 DMUs. For the second run of the BCC model, the same data set as the final CCR run (CCR4) was used. This will allow for the comparison of the $\mathrm{CCR}$ and $\mathrm{BCC}$ results at the start and end stages of the performance analysis.

\subsubsection{CORRELATION ANALYSIS}

The correlation values obtained for this model varied greatly, ranging from $-28 \%$ to $100 \%$. Several high correlations $(>95 \%)$ were observed suggesting that there are input and output variables that could be removed to improve the discriminatory power of the model. However, all variables were left in for symmetry and clarity of model purpose.

Low correlations $(<50 \%)$ were also observed between some of the input and output variables, namely the correlation values associated with 'existing customer growth' input variables. These low correlation values are most likely due to the large number of ' 0 ' place keepers associated with those DMUs who experience positive growth. Removing these variables was considered; however, exclusion from the model would negate the ability to penalize those branches who experienced losses in their 'existing customer' products. The complete correlation analysis can be observed in Figure 4.3. 


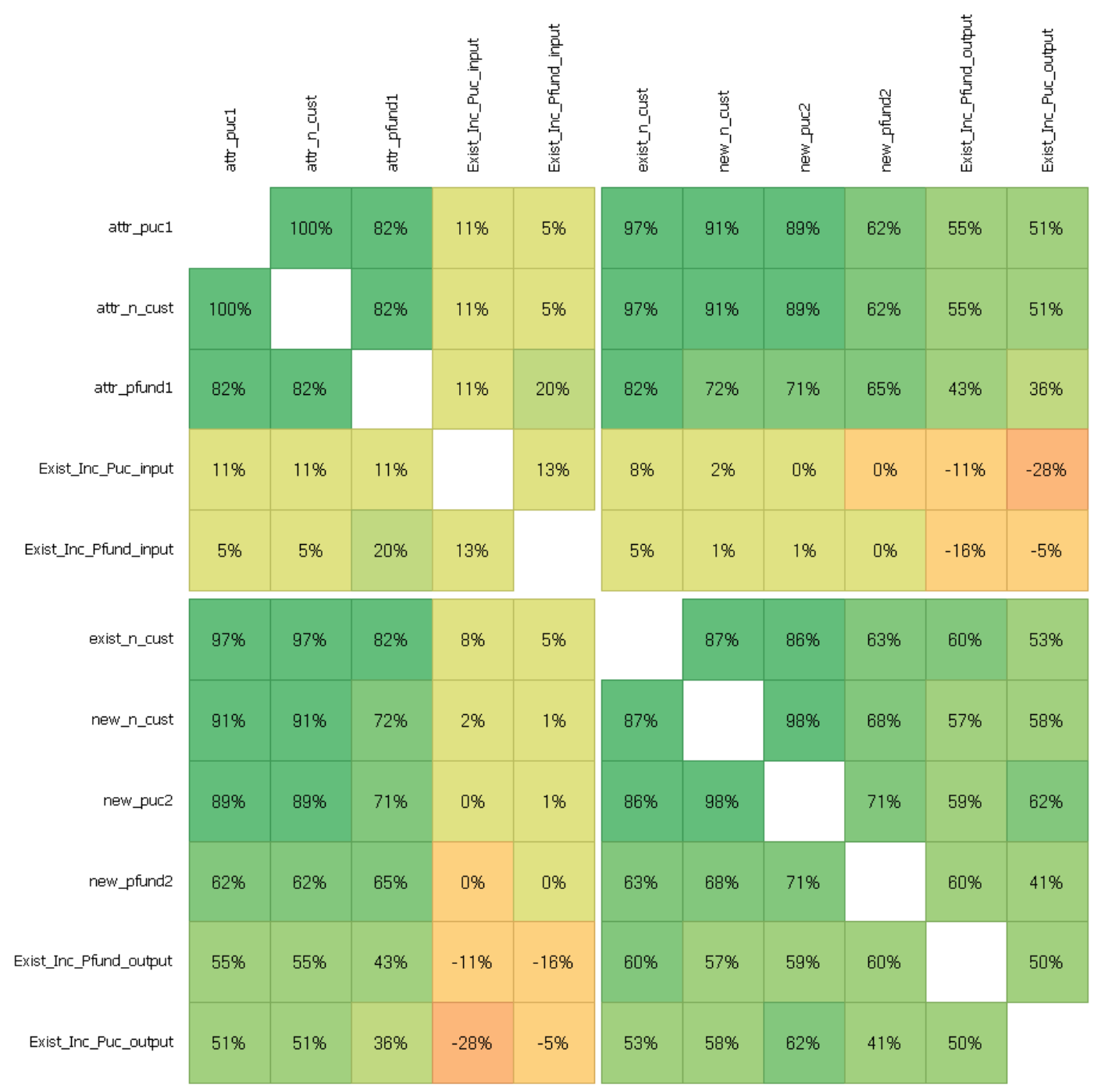

Figure 4.3: Lost Accounts/Gained Accounts Model- Correlation Analysis

\subsubsection{AVERAGE EFFICIENCY}

On the first run, the CCR model returned an average efficiency of 0.537 with a standard deviation of 0.11 and 11 efficient units. With the efficient units stripped and the CCR model rerun, an average efficiency of 0.601 with a standard deviation of 0.13 and 21 efficient units were observed. The third run returned an average efficiency of 0.673 with a standard deviation of 0.13 and 37 efficient units. The fourth and final CCR run produced an average efficiency of 0.723 with a standard deviation of 0.13 and 58 efficient units.

The first run of the BCC model returned an average efficiency of 0.763 with a standard deviation of 0.13 , while the second run average BCC score was 0.811 with a standard deviation of 0.12 . A summary of the model results is provided in Table 4.11. 
Table 4.11: Summary of Lost Accounts/Gained Accounts Model Average Efficiency Scores

\begin{tabular}{|c|c|c|c|c|c|c|}
\hline Run & \# of DMUs & $\begin{array}{c}\text { Average } \\
\text { Efficiency }\end{array}$ & Minimum & $\begin{array}{c}\text { \# of Efficient } \\
\text { DMUs }\end{array}$ & $\begin{array}{c}\text { \% of DMUs that } \\
\text { are Efficient }\end{array}$ & $\begin{array}{c}\text { Standard } \\
\text { Deviation }\end{array}$ \\
\hline \multicolumn{7}{|c|}{ CCR } \\
\hline 1 & 1041 & 0.537 & 0.285 & 11 & $1.06 \%$ & 0.111 \\
\hline 2 & 1030 & 0.601 & 0.326 & 21 & $2.04 \%$ & 0.130 \\
\hline 3 & 1009 & 0.673 & 0.400 & 37 & $3.67 \%$ & 0.132 \\
\hline 4 & 972 & 0.729 & 0.445 & 58 & $5.97 \%$ & 0.128 \\
\hline \multicolumn{7}{|c|}{ BCC } \\
\hline 1 & 1041 & 0.763 & 0.391 & 69 & $6.63 \%$ & 0.125 \\
\hline 2 & 972 & 0.811 & 0.449 & 125 & $12.86 \%$ & 0.121 \\
\hline
\end{tabular}

\subsubsection{MARKET}

When split by market, the Prairies had the highest average fourth run CCR score of 0.780 and GTA-East had the highest average second run BCC score of 0.850. Atlantic Canada had the lowest average CCR score of 0.693 while Southern Ontario had the lowest BCC score of 0.750. The standard deviations for both the CCR and BCC models were all in the range of 0.10 to 0.14 . A summary of these segmentation results are provided in Appendix 2.

\subsubsection{URBAN/RURAL}

When split into urban and rural, the rural market had the highest average fourth run CCR score (0.747), while the urban market had the highest average second run BCC score (0.817). It appears that the urban market fared much better under VRS assumptions while the rural market did much better under CRS assumptions.

\subsubsection{AsSET SIZE}

When split by funds managed, the Funds-S segment had the highest CCR mean efficiency of 0.743 and had the lowest BCC average efficiency score of 0.787 . Funds-XL had the lowest CCR efficiency score of 0.691 and had the highest BCC efficiency score of 0.901. The standard deviations varied between 0.1 and 0.15 .

\subsubsection{EXISTing Customer Base}

When split by customer, the Cust-S segment outperformed the other segments in the CCR model with an average efficiency score of 0.763 and scored the worst out of all segments in the BCC model with a score of 0.792. The Cust-XL segment outperformed the other segments in the BCC model with a score of 0.895 . For the CCR model, the segments in order of S to XL performed best to worst, while the exact opposite is true for the BCC model. Again, the standard 
deviations varied between runs, segments and models, with the largest being 0.16 and the smallest being 0.07 .

\subsubsection{RETURNS TO SCALE}

The scale efficiency of the first run was calculated to be 0.70 , suggesting that nonoptimal scale accounts for $30 \%$ of the overall inefficiency. The scale efficiency of the final run was found to be 0.90 , conceding that only $10 \%$ of inefficiencies are due to non-optimal scale, a $20 \%$ improvement. As with the "2010-2011 Period Growth" model, the majority of the branches were found to have decreasing returns to scale, with $99.5 \%$ in the first run and $93.9 \%$ in the final run. Constant returns to scale units were only found within the Funds-S segments, while IRS units were only present within the Funds-S and Funds-M segments.

\subsection{MARKet MODEL}

Assessing the relative growth efficiency of a bank's branches using metrics based purely on the DMUs internal operations provides the ability to better identify best performers within a branch network. However, it does not account for local economic and market conditions; both of which can have a large effect on a branch's performance. To better examine the Bank's performance relative to external market growth a Market model was developed and executed. This section introduces the "Market" model along with its objectives. The variable selection and model building processes are presented along with a synopsis of the obtained results.

\subsubsection{MOdEL OBJECTIVE}

Although the market model is relatively uncommon among bank branch efficiency analyses, it provides the opportunity to examine how branches are performing relative to their local market. The market model measures the extent to which a bank branch, given its capacity and available resources, realizes its potential to sell products and provide services in a given market. To achieve market efficiency, a bank branch must expand its outputs and optimize how cohesively the branch (size, number of employees, etc.) fits into its market conditions. The Market model presented herein examines a branch's ability to use its resources and local market conditions to grow its market share and produce new accounts.

The model building process began with clearly defining the purpose and viewpoints of the input and output variables. Input variables were defined as resources, branch characteristics and local market conditions, while output variables were defined as products and branch growth 
and market share. It follows that if a branch was operating with less resources or in worse market conditions and was able to produce the same number of accounts or attain the same market share as a branch with more resources or better market conditions, then the first branch would be considered more efficient. The finalized Market model can be viewed in Table 4.12.

Table 4.12: Market Model- Components

\begin{tabular}{|l|l|}
\hline \multicolumn{1}{|c|}{ Input } & \multicolumn{1}{c|}{ Output } \\
\hline \# of Customers (n_cust) & Total Lending Balance (Pldbl) \\
Total \# of MVC Clients (_ind_mvc) & Product Use Count (PUC) \\
Toal \# of Growable Clients (Ind_gro) & Total Deposit Accounts (Pdpbl) \\
\# of ABMs (_ABMs) & Total RRSP Balance (Pprbl) \\
Local Market- Total Funds Managed & Total Investment Balance (Pivbl) \\
(LMFM) & Branch's Share of Local Market (LMBS) \\
Branch Funds Managed Growth & Branch Funds Managed Growth \\
(TFM_Gr_input ) & (TFM_Gr_output) \\
Local Market Growth (LM_Gr_) & Total RRIF Balance (Prtbl) \\
& Funds Managed-Share of Local Market \\
& (LMFS) \\
\hline
\end{tabular}

The inputs consist of several self explanatory variables including number of customers, number of ABMs, local market growth, total funds managed by the local market and branch funds managed growth. Additionally, there are also input variables that are Bank specific, namely the total number of growable and MVC clients. Growable clients are those clients who have large external investments, loans or funds. These clients, if properly pursued, show great promise in opening additional accounts with the Bank. Most Valuable Clients (MVC) are clients that hold many products and a lot of funds with the bank. The outputs consist of the Bank's products along with branch growth and market share metrics. It should be noted that the "Branch Funds Managed Growth" variable was originally defined as an output variable, but as it includes both positive and negative values, it was split into an input and an output following the procedure defined in Section 4.6.1. It should be noted that for many of the aforementioned variables, data was only available for 2010 yearend, and thus this model was only executed for 2010 .

Among the selected variables, there exists variables that are considered to be uncontrollable (i.e. managers cannot control their values). These include the number of ABMs, the total funds managed by the local market and the local market growth. Restrictions were placed on these variables so that the DEA model could not change their quantities and so that all targets for these variables would equal their actual amount.

Combining variables to improve the discriminatory power and reduce the dimensionality of a DEA model is a common practice used in literature. To investigate the impact of reducing Multi-Stage Evaluation of Bank Branch Consolidation and Growth Potential Using DEA 56 
the number of variables, the individual products (i.e. Total deposits, investments, RRIFs, RRSPs and lending) were replaced with the Bank's 'funds managed' metric (equal to the sum of all products). This variable was introduced and used in all previous models. The reduced variable model is shown in Table 4.13. To maintain clarity, the original model is referred to as the "Component" Market model, while the reduced variable model is referred to as the "Aggregate" Market model.

Table 4.13: Market Model- Aggregate

\begin{tabular}{|l|l|}
\hline \multicolumn{1}{|c|}{ Input } & \multicolumn{1}{|c|}{ Output } \\
\hline \# of Customers (n_cust) & Product Use Count (PUC) \\
Total \# of MVC Clients (_ind_mvc) & Total Funds Managed (pfund) \\
Toal \# of Growable Clients (Ind_gro) & Branch's Share of Local Market (LMBS) \\
\# of ABMs (_ABMs) & Branch Funds Managed Growth \\
Local Market-Total Funds Managed & (TFM_Gr_output) \\
(LMFM) & Funds Managed-Share of Local Market \\
Branch Funds Managed Growth & (LMFS) \\
(TFM_Gr_input) & \\
Local Market Growth (LM_Gr_) & \\
\hline
\end{tabular}

\subsubsection{METHODOLOGY}

Unlike the "2010-2011 Period Growth" and "Lost Accounts/Gained Accounts" models, the Market models returned a large number of efficient units on their first run. As a result, the methodology was modified to include only one strip of the efficient frontier. Additionally, several DMUs were missing data associated with the market variables and needed to be removed from the model. The methodology applied to both the Component and Aggregate Market models is depicted below in Figure 4.4. All the three runs of the BCC model use the same data sets as the corresponding run in the CCR model; thus, the models can be compared at each stage. 

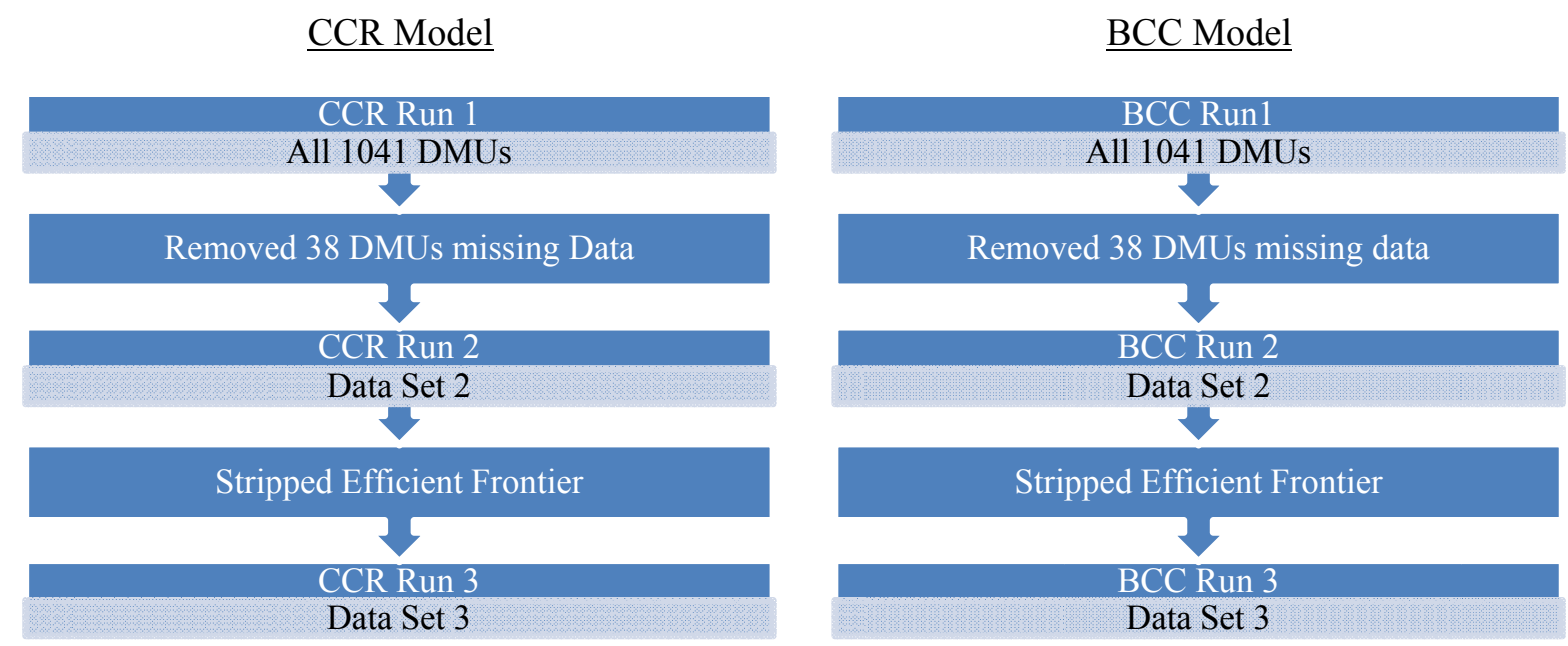

Figure 4.4: Market Model Methodology

\subsubsection{CORRELATION ANALYSIS}

For the Market model, an extensive correlation analysis was performed. Between the inputs only the number of customers and the number of growable clients had a correlation above $95 \%$. This correlation can be attributed to the fact that all branches have relatively the same percent of growable clients; larger branches will have more growable clients, and smaller branches will have fewer growable customers but the margins are approximately equal. Both variables were included in the model as they represent two distinct resources. Although many of the product- related variables show strong signs of correlation, none of their correlation coefficients rise above $95 \%$ and thus all of the outputs were left in as well.

Low correlations $(<50 \%)$ were observed for variables related to market conditions, market and branch growth and branch share of market. This may be related to the fact that most of these metrics are percentages and thus fall between 0 and 1 . It may also suggest that in reality these variables are independent of any branch characteristics. The complete correlation analysis can be observed in Figure 4.5. 


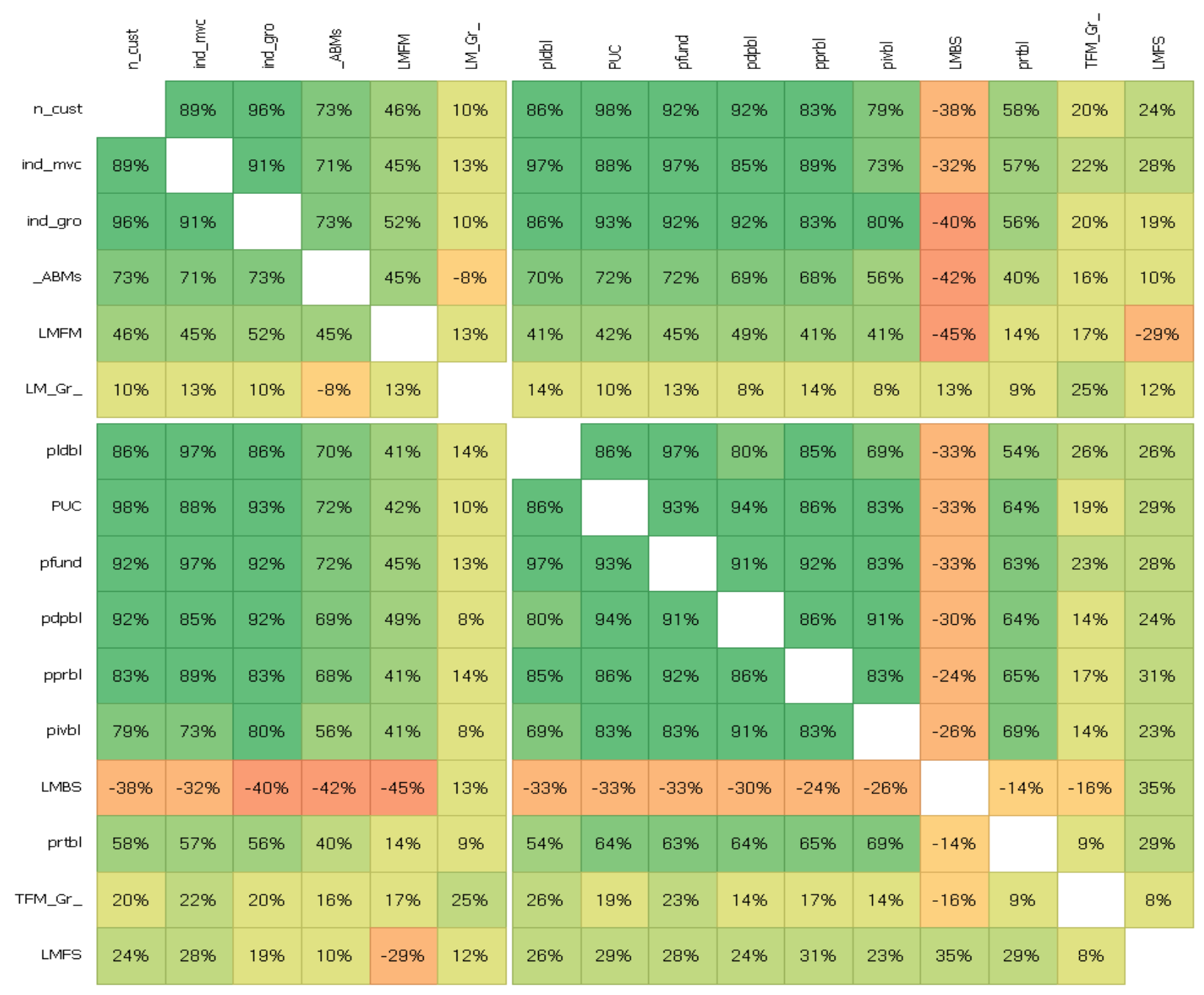

Figure 4.5: Market Model Correlation Analysis

\subsubsection{AVERAGE EFFICIENCY}

\subsubsection{COMPONENT MODEL}

On the first run, the CCR Component model returned an average efficiency of 0.922 with a standard deviation of 0.10 and 578 efficient units. With 38 units stripped, an average efficiency of 0.928 with a standard deviation of 0.01 and 603 efficient units were observed. In keeping with the methodology used with previous models, the efficient units were stripped returning an average efficiency of 0.983 with a standard deviation of 0.05 and 350 efficient units (88\%).

The results of the BCC Component model were very similar to those obtained from the CCR analysis. The first run returned an average efficiency score of 0.950 with a standard deviation of 0.09 and 760 efficient DMUs. The second execution of the model resulted in an average efficiency score of 0.951 with a standard deviation of 0.09 and 743 efficient units. The final run of the BCC model returned an average efficiency score of 0.989 with a standard 
deviation of 0.04 and 367 (91.8\%) efficient units. A summary of the results of the CCR and BCC Component models is provided in Table 4.14.

Table 4.14: Summary of Component Model Average Efficiency Results

\begin{tabular}{|c|c|c|c|c|c|c|}
\hline Run & $\begin{array}{c}\text { \# of } \\
\text { DMUs }\end{array}$ & $\begin{array}{c}\text { Average } \\
\text { Efficiency }\end{array}$ & Minimum & $\begin{array}{c}\text { \# of Efficient } \\
\text { DMUs }\end{array}$ & $\begin{array}{c}\% \text { of DMUs that } \\
\text { are Efficient }\end{array}$ & $\begin{array}{c}\text { Standard } \\
\text { Deviation }\end{array}$ \\
\hline \multicolumn{7}{|c|}{ CCR } \\
\hline 1 & 1041 & 0.922 & 0.592 & 578 & $55.52 \%$ & 0.100 \\
\hline 2 & 1003 & 0.928 & 0.592 & 603 & $60.12 \%$ & 0.099 \\
\hline 3 & 400 & 0.983 & 0.687 & 350 & $87.50 \%$ & 0.050 \\
\hline \multicolumn{7}{|c|}{ BCC } \\
\hline 1 & 1041 & 0.950 & 0.602 & 760 & $73.01 \%$ & 0.091 \\
\hline 2 & 1003 & 0.952 & 0.602 & 743 & $74.08 \%$ & 0.090 \\
\hline 3 & 400 & 0.989 & 0.690 & 367 & $91.75 \%$ & 0.042 \\
\hline
\end{tabular}

\subsubsection{AgGREgATE MODEL}

On the first run, the CRR model returned an average efficiency of 0.871 with a standard deviation of 0.11 and 317 efficient units. The second run returned an average efficiency of 0.880 with a standard deviation of 0.11 and 359 efficient units. This result (35\% efficient DMUs) closely follows literature's general consensus that approximately $30 \%$ of DMUs are efficient in when executing DEA branch analyses. The third run returned an average efficiency of 0.948 with a standard deviation of 0.08 and 417 (65\%) efficient units.

The first run of the BCC model returned an average efficiency score of 0.912 with a standard deviation of 0.11 and 561 efficient DMUs. The second execution of the model resulted in an average efficiency score of 0.917 with a standard deviation of 0.011 and 573 efficient units. The final run of the Aggregate model returned an average efficiency score of 0.965 with a standard deviation of 0.07 and 484 efficient units (75\%). A summary of the results of the Aggregate models is provided in Table 4.15.

Table 4.15: Summary of Aggregate Model Average Efficiency Results

\begin{tabular}{|c|c|c|c|c|c|c|}
\hline Run & $\begin{array}{c}\text { \# of } \\
\text { DMUs }\end{array}$ & $\begin{array}{c}\text { Average } \\
\text { Efficiency }\end{array}$ & Minimum & $\begin{array}{c}\text { \# of Efficient } \\
\text { DMUs }\end{array}$ & $\begin{array}{c}\text { \% of DMUs that } \\
\text { are Efficient }\end{array}$ & $\begin{array}{c}\text { Standard } \\
\text { Deviation }\end{array}$ \\
\hline \multicolumn{7}{|c|}{ CCR } \\
\hline 1 & 1041 & 0.871 & 0.576 & 317 & $30.45 \%$ & 0.110 \\
\hline 2 & 1003 & 0.880 & 0.576 & 359 & $35.79 \%$ & 0.111 \\
\hline 3 & 644 & 0.948 & 0.665 & 417 & $64.75 \%$ & 0.081 \\
\hline \multicolumn{7}{|c|}{ BCC } \\
\hline 1 & 1041 & 0.912 & 0.587 & 561 & $53.89 \%$ & 0.108 \\
\hline 2 & 1003 & 0.917 & 0.587 & 573 & $57.13 \%$ & 0.108 \\
\hline 3 & 644 & 0.965 & 0.676 & 484 & $75.16 \%$ & 0.070 \\
\hline
\end{tabular}




\subsubsection{MARKET}

Due to the large number of efficient units removed between the second and third run, the following sections provide the second run average efficiency scores as opposed to the third run average efficiency scores. This practice is continued for each segment.

\subsubsection{COMPONENT MODEL}

When split by market, Northern Ontario had the highest average second run CCR score of 0.950 and the highest average second run BCC score of 0.969. Vancouver Lower Mainland had the lowest average CCR score of 0.911, while Atlantic Canada had the lowest BCC score of 0.916. The standard deviations for the second run CCR model ranged from 0.08 to 0.11 , while the standard deviations of the BCC model were all in the range of 0.095 .

\subsubsection{AgGREGATE MODEL}

For the Aggregate model, the highest average second run CCR score of 0.907 was observed by Southern Ontario, while the highest BCC score of 0.941 was observed by the Prairies. The GTA-West segment returned the lowest CCR score of 0.846 and Atlantic Canada returned the lowest BCC score of 0.881. The standard deviations for both the CCR and BCC models were in the range of 0.10 to 0.12 .

\subsubsection{URBAN/RURAL}

\subsubsection{COMPONENT MODEL}

When split into urban and rural, the rural market had the highest average second run CCR and BCC score of 0.94 and 0.96 , respectively. The urban market returned a CCR score of 0.917 and a BCC score of 0.943. The standard deviations of all models were in the range of 0.090 .

\subsubsection{AgGregate Model}

Following the same trend as the Component model, the rural market outperformed the urban market in the Aggregate model as well. The rural market returned an average second run CCR score of 0.898 while the urban market returned a score of 0.862 . For the BCC model, the rural market received an average efficiency score of 0.931 and the urban market obtained a score of 0.904 . The standard deviations of all models were consistently in the range of 0.11 . 


\subsubsection{Asset SIZE}

\subsubsection{COMPONENT MODEL}

When split by funds managed, the Funds-XL segment consistency outperformed the other segments, receiving a CCR score of 0.988 and a BCC score of 1.0. It should, however, be noted that this segment had very few remaining DMUs in the second run and therefore the results are slightly misleading. The lowest CCR score of 0.926 was observed by the Funds-S segment, while the lowest BDD score of 0.946 was observed by the Funds-M segment.

\subsubsection{AgGregAte Model}

Very similar results were obtained for the Aggregate model. The Funds-XL segment dominated both the CCR and BCC, returning respective scores of 0.958 and 0.993 . The lowest CCR and BCC score of 0.864 and 0.900 were observed by the Funds-M segment. The standard deviations of the second run efficiency scores were much more realistic than those obtained for the component model; ranging from 0.8 to 0.12 .

\subsubsection{EXisting Customer Base}

\subsubsection{COMPONENT MODEL}

The results of the customer based segmentation were very similar to those observed when segmented by funds. The Cust-XL segment performed the best in both the CCR and BCC models with respective scores of 0.977 and 0.996 . The Cust-M segment performed the worst in both the CCR and BCC models with scores of 0.903 and 0.928 .

\subsubsection{AgGregate Model}

Again following the trend of the asset size segmentation, the Funds-XL segment dominated both the CCR and BCC returning respective scores of 0.945 and 0.990 . The lowest CCR and BCC score of 0.853 and 0.885 were observed by the Funds-M segment. The standard deviations of the second run efficiency scores ranged from 0.4 to 0.11 .

\subsubsection{RETURNS TO SCALE}

Unlike previous models, all of the runs of the CCR and BCC models use the same set of DMUs. Therefore, for a more accurate representation of the scale efficiency and the returns to scale distributions, each run is presented.

\subsubsection{COMPONENT MODEL}


The scale efficiencies of the first second and third runs were calculated to be $0.971,0.976$ and 0.994 , respectively. The associated inefficiencies due to non-optimal scale are provided in below Table 4.16.

Table 4.16: Component Model Scale Efficiency

\begin{tabular}{|c|c|c|}
\hline Run & Scale Efficiency & $\begin{array}{c}\text { Inefficiency due to } \\
\text { Non-Optimal Scale }\end{array}$ \\
\hline 1 & 0.971 & $2.9 \%$ \\
\hline 2 & 0.976 & $2.4 \%$ \\
\hline 3 & 0.994 & $0.6 \%$ \\
\hline
\end{tabular}

As with the other models, all three runs of the BCC component model indicated that the majority of branches were operating under decreasing returns to scale. Approximately 20-30\% of branches were found to be operating under IRS and the remaining 2-13\% were operating under CRS. When split by funds managed, it was found that the CRS, DRS and IRS branches were evenly distributed between the segments relative to their size; save for the Funds-XL segment whose branches were operating solely under DRS. A complete summary of the returns to scale results for the Component models can be found in Appendix 2.

\subsubsection{AgGregate Model}

For the Aggregate model, the scale efficiencies of the first second and third runs were calculated to be $0.954,0.959$ and 0.983 , respectively. The associated inefficiencies due to nonoptimal scale are provided in below Table 4.17.

Table 4.17: Aggregate Model Scale Efficiency

\begin{tabular}{|c|c|c|}
\hline Run & Scale Efficiency & $\begin{array}{c}\text { Inefficiency due to } \\
\text { Non-Optimal Scale }\end{array}$ \\
\hline 1 & 0.954 & $4.6 \%$ \\
\hline 2 & 0.959 & $4.1 \%$ \\
\hline 3 & 0.983 & $1.7 \%$ \\
\hline
\end{tabular}

The distribution of CRS, IRS and DRS branches was similar to that found for the Component model with 2-9\% of branches being CRS, 20-30\% IRS and 80-60\% DRS. When split by funds managed, it was found that all segments had an even distribution of CRS, IRS and DRS branches relative to their segment size. 


\subsection{Delta Model}

The Delta model combines aspects of the aforementioned models to assess the branch growth realized over the 2010-2011 period. The model employs delta values, calculated using several of the previously introduced variables, to assess a branch's ability to grow customers, funds and products. This section introduces the "Delta" model's objective, variable selection process and the final model. Subsequently, a synopsis of the obtained results is provided.

\subsubsection{MODEL OBJECTIVE}

Although the "2010-2011 Period Growth" model was useful in examining the overall growth of products, funds and customers, it was unable to account for the growth of retained customers due to the lack of both 2010 and 2011 values. However, the Bank did provide variables that measured the change in the existing customer PUC and funds between 2010 and 2011. This allowed for the construction of a model that considered overall growth as well as the growth of existing clients.

Using the 2010 and 2011 data, the delta values $(\Delta$ value $=2011$ value -2010 value $)$ were calculated for the number of customers, PUC and funds managed. Delta values for the growth in PUC and funds managed of existing customers were provided by the Bank. As branches strive to maximize these delta values, all variables were placed as outputs. To hold the place of the inputs, a dummy variable was used. The final delta model is presented in Table 4.18.

Table 4.18: Delta Model

\begin{tabular}{|l|l|}
\hline \multicolumn{1}{|c|}{ Input } & \multicolumn{1}{|c|}{ Output } \\
\hline $\mathrm{DV}(=1)$ & Customers (cust) \\
& PUC (puc) \\
& Funds Managed (pfund) \\
& Existing Customer PUC (exist_puc) \\
& Existing Customer Funds Managed (exist_pfund) \\
\hline
\end{tabular}

A second Delta model that used the percent change of each variable was also constructed (Percent Change $=[2011$ value-2010value $] / 2010$ value). This was done to investigate whether removing the differences in scale would affect the outcome of the model. Let us consider two branches; a large branch and a small branch. Say the large branch, with 1,000 customers, gains 200 customers and the small branch, with 100 customers, gains 80 . Relative to the larger branch the smaller branch was outperformed, however, relative to its own size, the small branch performed much better than the larger branch. By using percent changes, all variable values will fall between 0 and 1; thus eliminating scale differences. It should be noted that there are risks 
associated with using percentages in DEA and the results of this model must therefore be carefully considered. For clarity's sake, the model using delta values is referred to as the "Difference" model, while the model using percentage change is referred to as the "Percent" model.

When constructing the model, special treatment of the data was required to deal with the presence of negative values associated with the existing customer delta values. Initially, the same methodology as that applied in the "Lost Accounts/Gained Accounts" model was used. However, this was found to be insufficient as there were very few negatives and thus an over abundance of ' 0 ' place holders on input side. As this model is translation invariant, a second commonly used methodology was employed where the absolute value of the largest negative of that variable was added to each DMU. This was done for both existing customer PUC and existing customer funds managed.

The use of a dummy variable input also required some consideration as it scaled the data resulting in equal results from both the $\mathrm{CCR}$ and $\mathrm{BCC}$ models; thus, only the CCR model was run. Moreover, the combination of freely moving multipliers and the dummy variable input makes the use of the CCR model less than ideal. In this case, the CCR DEA model would essentially give weights of zero to the lowest output scores and simply maximize the highest output score. Given the slacks values were relatively significant, the SBM approach outlined in [COOP07] was used to provide a more representative measure of efficiency. This resulted in the same number of efficient DMUs as the CCR model; however, the efficiency scores were derived purely from slacks.

\subsubsection{METHODOLOGY}

To begin, the same methodology that was used for the 2010-2011 Period Growth and Lost Accounts/Gained Accounts models, described in Figure 4.1, was used for both the Delta models. Upon observing the results, it was found that stripping the efficient frontier was not the most suitable method for these models and a second methodology employing statistical data analysis was also performed.

For each model, the coefficients of variation were calculated for each variable. The distribution of the variable with the highest coefficient was then analyzed using a histogram. For the Difference model, the 'existing customer PUC' variable was chosen, while the 'number of customers' variable was chosen for the Percent model. The histograms for each model are shown in Figures 4.6 and 4.7. 


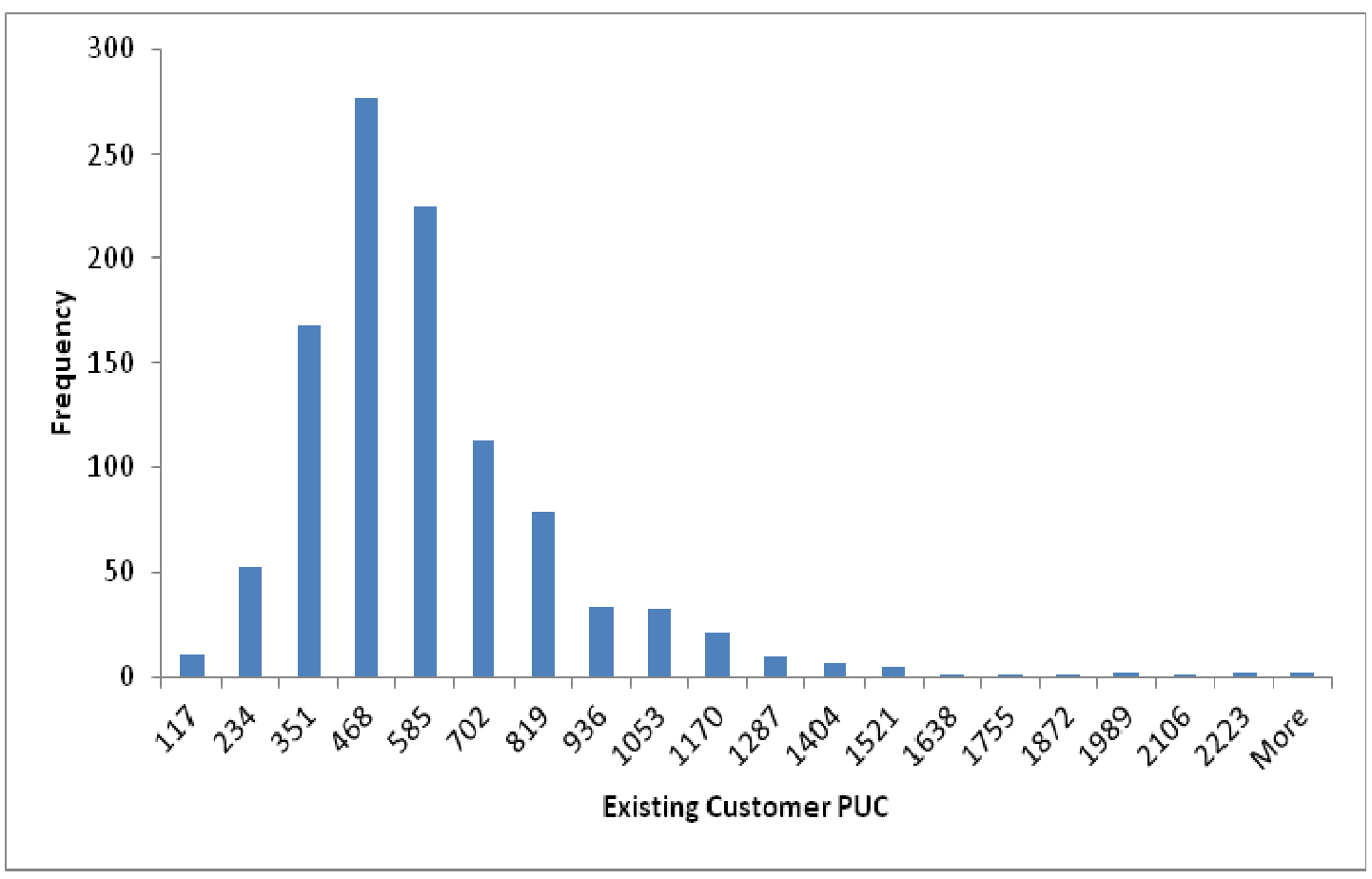

Figure 4.6: Difference Model- Existing Customer PUC Histogram

As shown, there is a very small number of DMUs that sit within the upper range of the distributions. Consequently, DMUs with an existing customer PUC value greater than 1375 were removed from the Difference model's data set. This resulted in the removal of a total of 20 DMUs.

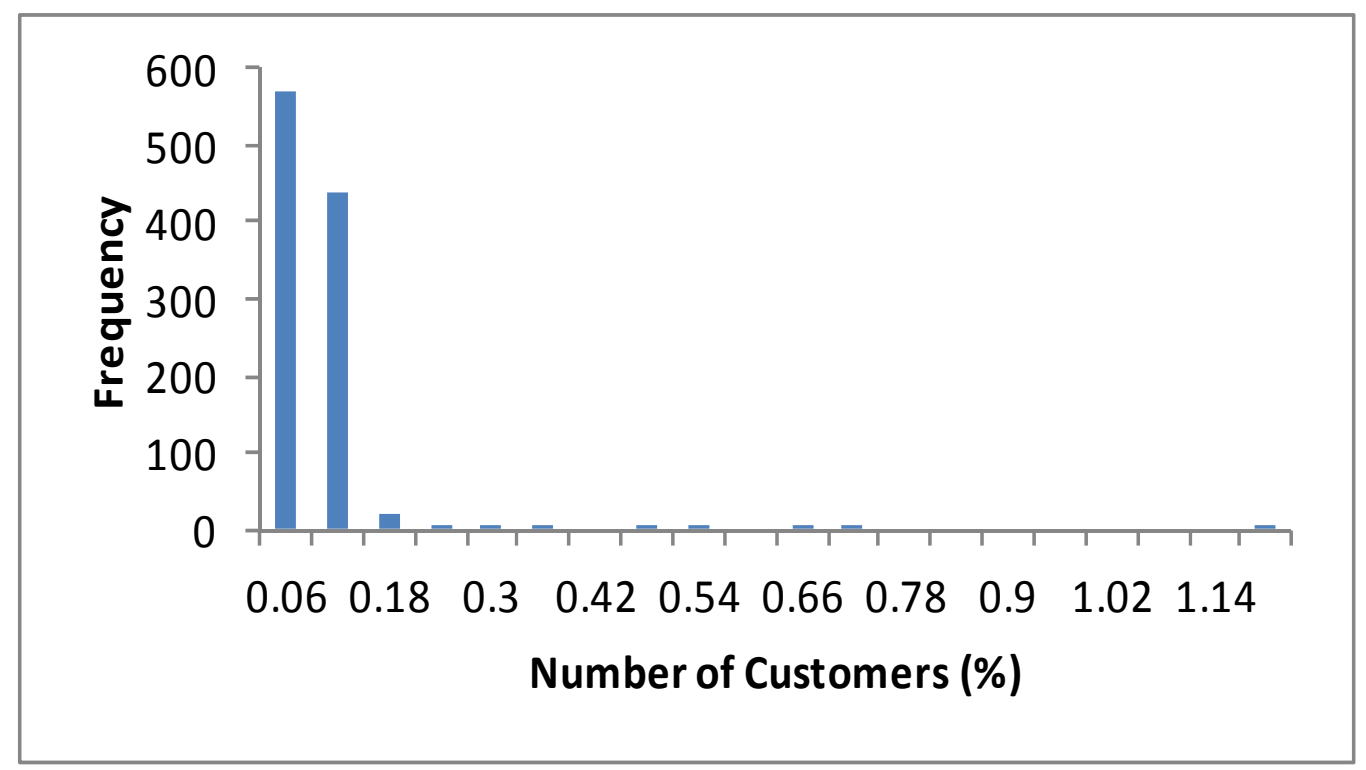

Figure 4.7: Percent Model- Number of Customers Histogram 
The distribution for the Percent model's 'number of customers' variable presented an even more drastic distribution with 1008 of the 1041 units falling between 0 and 0.12 . DMUs with values of 0.18 or greater, a total of 21 units, were removed. Once the DMUs were removed from each model, the CCR and SBM forms were run using the new data sets.

Local analysis using the full data set of 1041 units was also performed for each model. Instead of segmenting directly by market, customer base or funds managed, unique segments were calculated for both the Difference and Percent models based on the coefficients of variation of each variable. The coefficient of variation is a normalized measure of the dispersion of a variable with respect to the mean of its population. It is calculated by dividing the standard deviation by the mean. Higher coefficients of variation denote that there is more variation in the variable values between each DMU.

To account for the high volatility of certain variables, k-means segmentations were employed using the two variables with the highest coefficients of variation. For the Difference model, PUC and Existing PUC were used, while number of customers and funds managed were used for the Percent model. Once the first run of the local analysis was performed, the efficient frontier of each was stripped and re-run.

The complete procedure for the "Data Analysis and Local DEA" methodology is depicted in Figure 4.8.
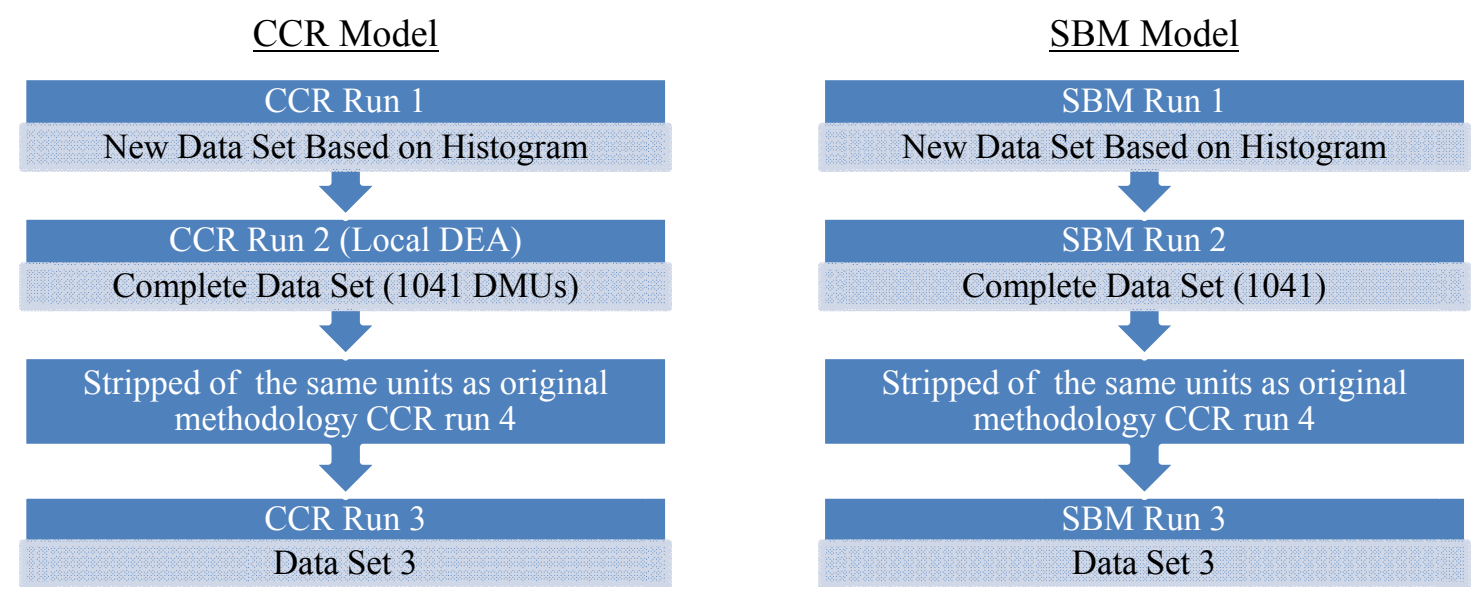

Figure 4.8: Delta Model 'Data Analysis and Local DEA' Execution Methodology

For clarity, the original methodology is referred to as "stripping efficient units" and the new methodology is referred to as "data analysis and local DEA". The results of the Difference model and Percent model using both methodologies are presented in the following sections. 


\subsubsection{CORRELAtion AnAlysis}

Due to the use of a dummy variable input, correlation analysis could only be performed between outputs and the relevance indices could not be calculated. The following sections provide a brief discussion of the correlation analyses performed for the Difference and Percent models.

\subsubsection{DIFFERENCE MODEL}

The Difference model correlation analysis showed no outputs with overly high correlations ( $>95 \%)$. The highest observed correlation was $89 \%$ while the lowest was $35 \%$. The remaining correlations fell within the range of $47 \%-78 \%$. This analysis suggests that there is little to no redundancy in the information provided by the variables, and thus, all variables should remain in the model. A summary of the correlation analysis is provided in Figure 4.9.

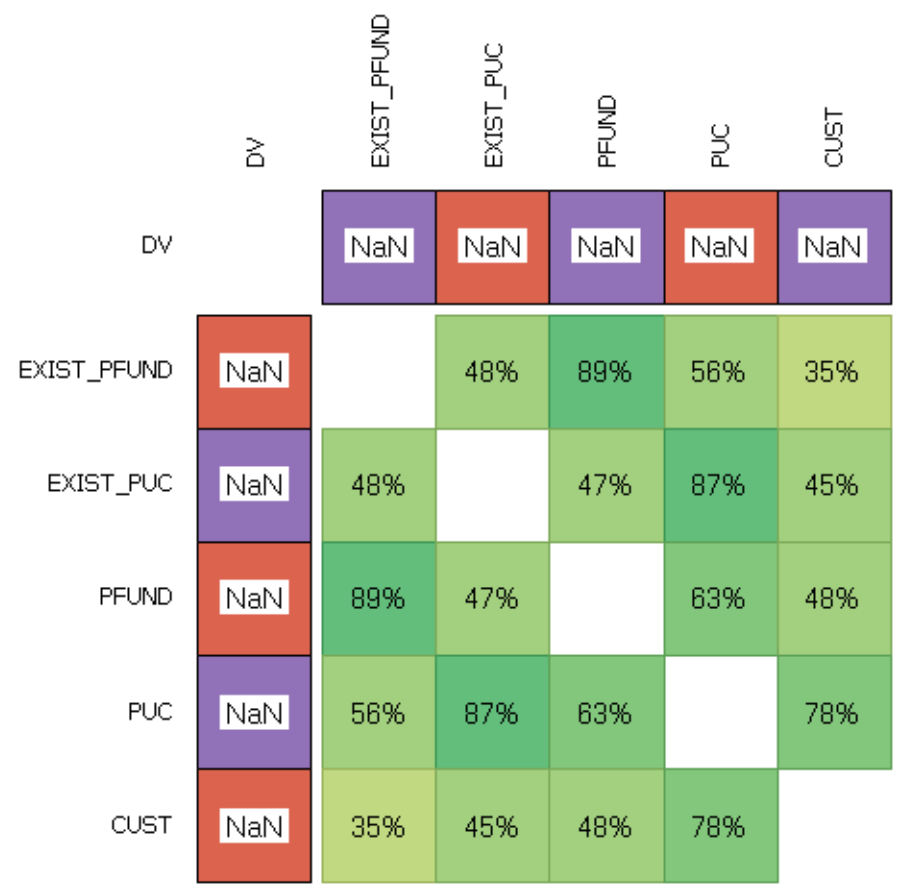

Figure 4.9: Delta Model (Difference)- Correlation Analysis

\subsubsection{PERCENT MODEL}

The Percent model correlation analysis showed similar results to that of the Difference model but with a few slightly higher values. The highest observed correlation was $94 \%$ while the lowest was $45 \%$. Again, the moderate correlation coefficients tend to suggest that the variables should all remain in the model. A summary of the correlation analysis is provided below in Figure 4.10. 


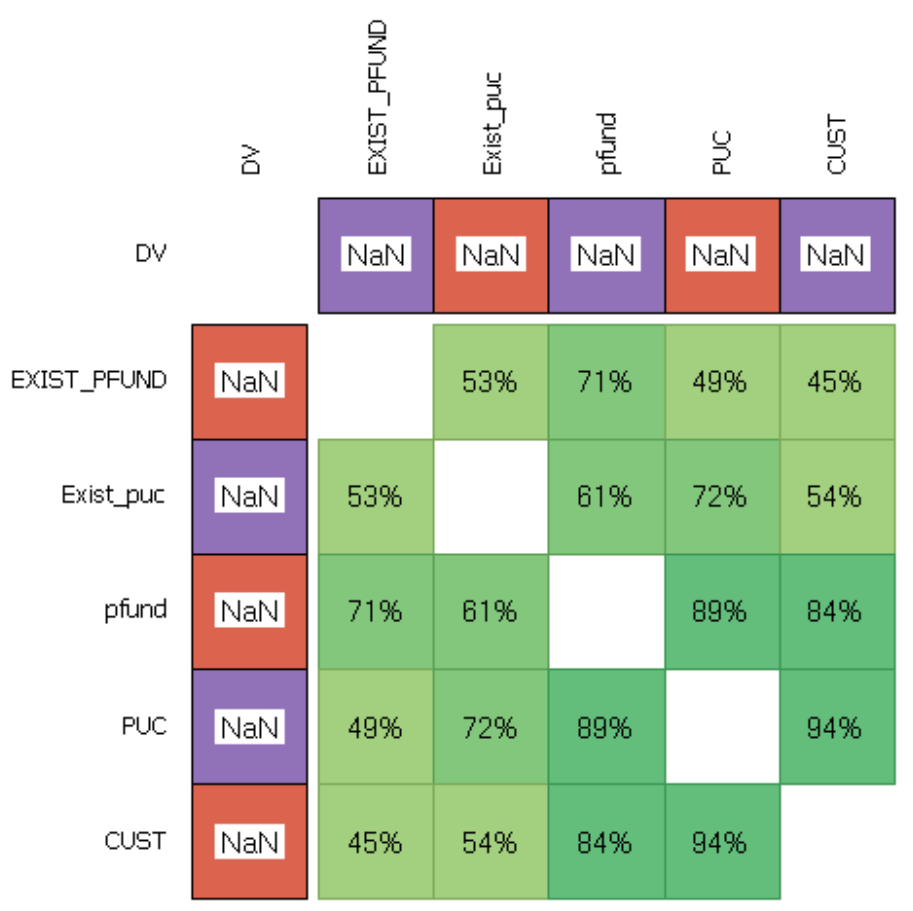

Figure 4.10: Delta Model (Percent)- Correlation Analysis

\subsubsection{DifFERENCE MODEL}

\section{By Stripping Efficient Units}

The first run of the CCR Difference model returned an average efficiency score of 0.36 with a standard deviation of 0.12 and only 4 efficient DMUs. The second execution of the model resulted in an average efficiency score of 0.50 with a standard deviation of 0.13 and 11 efficient units. The third run of the CCR model returned an average efficiency score of 0.55 with a standard deviation of 0.13 and 11 efficient units. The final run returned an efficiency score of 0.60 with a standard deviation of 0.14 and 17 efficient units.

The first run of the SBM Difference model returned an average efficiency score of 0.23 with a standard deviation of 0.08 and 4 efficient DMUs. The second execution of the model resulted in an average efficiency score of 0.488 with a standard deviation of 0.15 and 17 efficient units. As expected, the SBM model returned lower efficiency scores than the CCR model. A summary of the results obtained from the CCR and SBM models is provided in Table 4.19.

Banks are highly regulated and their operations are relatively consistent, resulting in similar efficiency scores. The presence of very low efficiency scores suggests there is a group of outliers that is dominating the data set and heavily skewing the results. 
Table 4.19: Summary of Difference Model Efficiency Results- Stripping

\begin{tabular}{|c|c|c|c|c|c|c|}
\hline Run & \# of DMUs & $\begin{array}{c}\text { Average } \\
\text { Efficiency }\end{array}$ & Minimum & $\begin{array}{c}\text { \# of Efficient } \\
\text { DMUs }\end{array}$ & $\begin{array}{c}\text { \% of DMUs that } \\
\text { are Efficient }\end{array}$ & $\begin{array}{c}\text { Standard } \\
\text { Deviation }\end{array}$ \\
\hline \multicolumn{7}{|c|}{ CCR } \\
\hline 1 & 1041 & 0.361 & 0.187 & 4 & $0.38 \%$ & 0.116 \\
\hline 2 & 1037 & 0.504 & 0.311 & 11 & $1.06 \%$ & 0.130 \\
\hline 3 & 1026 & 0.544 & 0.326 & 11 & $1.07 \%$ & 0.130 \\
\hline 4 & 1015 & 0.604 & 0.348 & 17 & $1.67 \%$ & 0.136 \\
\hline \multicolumn{7}{|c|}{ SBM } \\
\hline 1 & 1041 & 0.230 & 0.064 & 4 & $0.38 \%$ & 0.084 \\
\hline 2 & 1015 & 0.488 & 0.103 & 17 & $1.67 \%$ & 0.148 \\
\hline
\end{tabular}

By Data Analysis and Local DEA

The results obtained by using data analysis and local analysis changed the results of the CCR model quite drastically. The first run, with the removal of 20 extreme valued DMUs, returned an average efficiency score of 0.51 with a standard deviation of 0.14 and 9 efficient units. Run two, which used local analysis, resulted in an average efficiency score of 0.85 with a standard deviation of 0.09 and 58 efficient units. Compared to the original methodology, local analysis returned far more efficient units and a much higher average efficiency score. The final run returned an average efficiency score of 0.86 with a standard deviation of 0.09 and 68 efficient units.

As observed above, using data analysis and local analysis on the SBM model also returned higher average efficiency scores and more efficient units. The first run, with the removal of 20 extreme valued DMUs, returned an average efficiency score of 0.31 with a standard deviation of 0.11 and 9 efficient units. Run two, which used local analysis, resulted in an average efficiency score of 0.76 with a standard deviation of 0.14 and 58 efficient units. The final run returned an average efficiency score of 0.79 with a standard deviation of 0.12 and 68 efficient units. A summary of these results obtained for the CCR and SBM models is provided in Table 4.20.

Table 4.20: Summary of Difference Model Efficiency Results- Data Analysis and Local DEA

\begin{tabular}{|c|c|c|c|c|c|c|}
\hline Run & \# of DMUs & $\begin{array}{c}\text { Average } \\
\text { Efficiency }\end{array}$ & Minimum & $\begin{array}{c}\text { \# of Efficient } \\
\text { DMUs }\end{array}$ & $\begin{array}{c}\% \text { of DMUs that } \\
\text { are Efficient }\end{array}$ & $\begin{array}{c}\text { Standard } \\
\text { Deviation }\end{array}$ \\
\hline \multicolumn{7}{|c|}{ CCR- Statistical Analysis } \\
\hline 1 & 1021 & 0.510 & 0.268 & 9 & $0.88 \%$ & 0.144 \\
\hline \multicolumn{7}{|c|}{ CCR- Local Analysis } \\
\hline 2 & 1041 & 0.845 & 0.568 & 58 & $5.57 \%$ & 0.093 \\
\hline 3 & 1015 & 0.855 & 0.605 & 68 & $6.70 \%$ & 0.090 \\
\hline \multicolumn{7}{|c|}{ SBM- Statistical Analysis } \\
\hline 1 & 1021 & 0.311 & 0.095 & 9 & $0.88 \%$ & 0.113 \\
\hline 2 & 1041 & 0.764 & 0.231 & 58 & $5.57 \%$ & 0.135 \\
\hline 3 & 1015 & 0.789 & 0.231 & 68 & $6.70 \%$ & 0.118 \\
\hline
\end{tabular}




\subsubsection{Percent ModeL}

\section{By Stripping Efficient Units}

Much like the Difference model, the first run of the CCR Percent model returned a low average efficiency score of 0.31 with a standard deviation of 0.08 and only 1 efficient DMU (Unit 508). The second execution of the model resulted in an average efficiency score of 0.41 with a standard deviation of 0.10 and 6 efficient units. The third run of the CCR model returned an average efficiency score of 0.52 with a standard deviation of 0.11 and 6 efficient units. The final run of the CCR model returned an average efficiency score of 0.56 with a standard deviation of 0.12 and 9 efficient units.

The first run of the SBM Percent model returned an average efficiency score of 0.10 with a standard deviation of 0.05 and the same efficient unit (508) identified by the CCR model. The second execution of the model resulted in an average efficiency score of 0.36 with a standard deviation of 0.11 and 9 efficient units. A summary of the results of the CCR and SBM models is provided in Table 4.21 .

Table 4.21: Summary of Percent Model Efficiency Results- Stripping

\begin{tabular}{|c|c|c|c|c|c|c|}
\hline Run & \# of DMUs & $\begin{array}{c}\text { Average } \\
\text { Efficiency }\end{array}$ & Minimum & $\begin{array}{c}\text { \# of Efficient } \\
\text { DMUs }\end{array}$ & $\begin{array}{c}\text { \% of DMUs that } \\
\text { are Efficient }\end{array}$ & $\begin{array}{c}\text { Standard } \\
\text { Deviation }\end{array}$ \\
\hline \multicolumn{7}{|c|}{ CCR } \\
\hline 1 & 1041 & 0.307 & 0.030 & 1 & $0.10 \%$ & 0.082 \\
\hline 2 & 1040 & 0.413 & 0.054 & 6 & $0.58 \%$ & 0.104 \\
\hline 3 & 1034 & 0.519 & 0.072 & 6 & $0.58 \%$ & 0.114 \\
\hline 4 & 1028 & 0.559 & 0.111 & 9 & 0.875 & 0.116 \\
\hline \multicolumn{7}{|c|}{ SBM } \\
\hline 1 & 1041 & 0.095 & 0.005 & 1 & $0.10 \%$ & 0.051 \\
\hline 2 & 1028 & 0.364 & 0.016 & 9 & $0.88 \%$ & 0.113 \\
\hline
\end{tabular}

\section{By Data Analysis and Local DEA}

Again, a large difference was observed when the CCR model was re-run using data analysis and local DEA. The first run of the CCR model, with the removal of 21 extreme valued DMUs, returned an average efficiency score of 0.54 with a standard deviation of 0.11 and 10 efficient units. Run two, which used local analysis, resulted in an average efficiency score of 0.78 with a standard deviation of 0.13 and 31 efficient units. The final run returned an average efficiency score of 0.79 with a standard deviation of 0.13 and 34 efficient units.

When using data analysis and local DEA, the first run of the SBM Percent model with 21 DMUs removed returned an average efficiency score of 0.42 with a standard deviation of 0.11 and 10 efficient units. Run two, which used local analysis, resulted in an average efficiency score 
of 0.66 with a standard deviation of 0.13 and 30 efficient units. The final run returned an average efficiency score of 0.67 with a standard deviation of 0.13 and 33 efficient units. A summary of these results obtained from both the CCR and SBM models is provided in Table 4.22.

Table 4.22: Summary of Percent Model Efficiency Results- Data Analysis and Local DEA

\begin{tabular}{|c|c|c|c|c|c|c|}
\hline Run & \# of DMUs & $\begin{array}{c}\text { Average } \\
\text { Efficiency }\end{array}$ & Minimum & $\begin{array}{c}\text { \# of Efficient } \\
\text { DMUs }\end{array}$ & $\begin{array}{c}\text { \% of DMUs that } \\
\text { are Efficient }\end{array}$ & $\begin{array}{c}\text { Standard } \\
\text { Deviation }\end{array}$ \\
\hline \multicolumn{7}{|c|}{ CCR- Statistical Analysis } \\
\hline 1 & 1021 & 0.541 & 0.233 & 10 & $0.98 \%$ & 0.114 \\
\hline \multicolumn{7}{|c|}{ CCR- Local Analysis } \\
\hline 2 & 1041 & 0.778 & 0.281 & 31 & $3.00 \%$ & 0.128 \\
\hline 3 & 1028 & 0.785 & 0.281 & 34 & $3.31 \%$ & 0.125 \\
\hline \multicolumn{7}{|c|}{ SBM- Statistical Analysis } \\
\hline 1 & 1021 & 0.416 & 0.016 & 10 & $0.98 \%$ & 0.111 \\
\hline 2 & 1041 & 0.660 & 0.052 & 30 & $2.90 \%$ & 0.132 \\
\hline 3 & 1028 & 0.671 & 0.052 & 33 & $3.21 \%$ & 0.128 \\
\hline
\end{tabular}

\subsubsection{MARKET}

\subsubsection{DifFERENCE MOdEL: STRIPPING EFFICIENT UNITS}

When segmented by market, GTA-East and Vancouver Lower Mainland had the highest average fourth run CCR scores of 0.667; while GTA-East alone had the highest SBM score of 0.565. The lowest average CCR (0.536) and SBM (0.428) efficiency scores were observed by Southern Ontario. The standard deviations for both the CCR and SBM models were in the range of 0.12 to 0.15 . A summary of the segmentation results are provided in Appendix 2 .

\subsubsection{DifFEREnCE Model: Data ANalysis AND Local DEA}

When the Difference model was treated with data analysis and local DEA, the highest average third run CCR (0.886) and SBM (0.821) scores were observed by Vancouver Lower Mainland. While the lowest average third run CCR score of 0.829 and the lowest SBM score of 0.756 were observed by Atlantic Canada. The standard deviations for the CCR model were all in the range of 0.9 , and 0.12 for the SBM model.

\subsubsection{Percent Model: StRIPPINg EFFICIENT Units}

When the Percent model, treated with stripping, was segmented by market, the GTAEast, again, had the highest average fourth run CCR score of 0.607 and SBM score of 0.400. The lowest average CCR (0.485) and SBM (0.315) efficiency scores were observed by Atlantic Canada. The standard deviations for the third run CCR model were all in the ranged from 0.09 to 0.13 , while the standard deviations of the SBM model ranged between 0.05 and 0.11 . 


\subsubsection{PERCENT MOdel: DATA ANALYSIS AND LOCAL DEA}

When the Percent model was treated with data analysis and local DEA, the highest average third run CCR (0.802) and SBM (0.696) scores were observed by Quebec, while the lowest average third run CCR score of 0.741 and the lowest SBM score of 0.624 were observed by Atlantic Canada. The standard deviations for the CCR model ranged from 0.12 to 0.14 , and were all in the 0.12 for the SBM model.

\subsubsection{URBAN/RURAL}

\subsubsection{DifFERENCE MODEL: STRIPPING EFFICIENT UNITS}

When split into urban and rural, the urban market had the highest CCR and SBM average third run efficiency scores of 0.653 and 0.537 , respectively. The rural market returned an average third run CCR score of 0.549 and an average SBM score of 0.435 .

\subsubsection{DifFerence Model: Data ANALysis AND LOCAl DEA}

When the Difference model, treated with data analysis and local DEA, was segmented into urban and rural, the urban market again outperformed the rural market. The Urban market returned a CCR score of 0.872 and an SBM score of 0.802 . The rural market returned an average third run CCR score of 0.837 and an average SBM score of 0.774 .

\subsubsection{Percent Model: Stripping EFFicient Units}

When the Percent model was split into urban and rural, the results followed the same trend as above. The Urban market outperformed the rural market, returning a CCR score of 0.570 and an SBM score of 0.375 . The rural market returned an average third run CCR score of 0.546 and an average SBM score of 0.352 .

\subsubsection{Percent Model: Data ANALysis AND Local DEA}

Again, when the Percent model, treated with data analysis and local DEA, was segmented into urban and rural, the urban market returned a higher average efficiency than the rural market. The Urban market produced an average third run CCR score of 0.787 and an average third run SBM score of 0.675 . The rural market returned an average third run CCR score of 0.782 and an average SBM score of 0.668 . 


\subsubsection{ASSET SIZE}

\subsubsection{DifFERENCE MODEL: STRIPPING EFFICIENT UNITS}

When split by funds managed, the Funds-XL segment returned the highest average CCR efficiency score of 0.801 and the highest SBM efficiency score of 0.651 . The Funds-S segment performed the worst in both the CCR and SBM models with respective scores of 0.547 and 0.437. The standard deviations varied between runs, segments and models, with the Funds-XL segment having the highest standard deviations of 0.28 . In general the standard deviations observed from the SBM model were higher than those of the CCR model.

\subsubsection{Difference Model: Data ANALysis AND Local DEA}

Similar results were obtained when for the Difference model that was treated with data analysis and local DEA. In this case, the Funds-XL segment returned the highest average efficiency scores for both the CCR (0.971) and SBM (0.892) models. Likewise, the Funds-S segment performed the worst with a CCR score of 0.838 and a SBM score of 0.776. The standard deviations of the third run CCR score were all in the range of 0.8 while those of the SBM model ranged from 0.11 to 0.15 .

\subsubsection{Percent Model: StRIPPING EFFICIENT Units}

When segmented by funds, the Percent model returned results that differed greatly from those reported by the Difference model. In the Percent model, the Funds-S produced the highest average CCR (0.574) and SBM (0.337) efficiency scores. The lowest CCR (0.510) and SBM (0.337) scores were realized by the Funds-XL segment.

\subsubsection{Percent Model: Data ANALysis AND LocAl DEA}

When the Percent model, treated with data analysis and local DEA, was segmented by funds, it also produced opposing results to those obtained by the Difference model. The FundsXL segment performed the best in the CCR model, with an average efficiency score of 0.807 , while the Funds-S segment performed the best in the SBM model producing a score of 0.674. The lowest average CCR score of 0.779 was returned by the Funds-M segment while the lowest SBM score of 0.659 was returned by the Funds-XL segment. 


\subsubsection{EXisting Customer Base}

\subsubsection{DifFERENCE MODEL: STRIPPING EFFICIENT UNITS}

When segmented by customer, the Cust-XL segment performed the best in the CCR model with an average efficiency score of 0.819 and the best in the SBM model with a score of 0.673. The Cust-S returned the lowest scores with a CCR score of 0.533 and a SBM score of 0.420. For both models, the segments performed best to worst in order of largest to smallest. The standard deviations for the CCR model varied between 0.9 and 0.16 while those of the SBM model varied between 0.09 and 0.23 .

\subsubsection{DifFERENCE MODEL: DATA ANALYSIS AND LOCAL DEA}

When treated with data analysis and local DEA, the Difference model followed the same trend as depicted above. For both the CCR and SBM models, the segments performed best to worst in order of largest to smallest. The Cust-XL segment returned a CCR score of 0.938 and an SBM score of 0.845 , while the Cust-S segment returned a CCR score of 0.832 and an SBM score of 0.772 . The standard deviations observed for the CCR model, all within the range of 0.09 , were lower than those observed for the $\operatorname{SBM}(0.10$ to 0.15$)$.

\subsubsection{Percent Model: StRIPPING EFFICIENT Units}

As with segmentation by funds, the results obtained for segmentation by customer for the Percent model differed greatly from those obtained for the Difference model. The Cust-S produced the highest average CCR (0.571) and SBM (0.374) efficiency scores. The lowest CCR (0.538) and SBM (0.340) scores were realized by the Cust-XL segment.

\subsubsection{Percent Model: Data Analysis And Local DEA}

When the Percent model, treated with data analysis and local DEA, was segmented by customers the Cust-XL segment performed the best in the CCR model and SBM models returning respective scores of 0.811 and 0.685 . The lowest CCR and SBM scores of 0.774 and 0.664 were both realized by the Cust-M segment.

\subsubsection{RETURNS TO SCALE}

As the CCR and BCC models were found to be equal for both the Difference and Percent models, the scale efficiency of all the models was calculated to be 1 . It follows that under this model, all DMUs operate under constant returns to scale. The methodologies applied to the Delta models also offered the unique opportunity to investigate the mix efficiencies of each model. The 
mix efficiency is calculated by dividing the SBM score by the CCR score and can range from 0 to 1 . A mix efficiency of 1 infers that the CCR model has produced optimal solutions with no slacks. A summary of the mixed efficiencies obtained for the Delta models is provided in Table 4.23 .

Table 4.23: Summary of the Mix Efficiency Score for the Delta Models

\begin{tabular}{|l|c|}
\hline \multicolumn{1}{|c|}{$\begin{array}{c}\text { Data Treatment } \\
\text { Methodology }\end{array}$} & Mix Efficiency \\
\hline \multicolumn{2}{|c|}{ Difference Model } \\
\hline Stripping the Frontier & $80.90 \%$ \\
\hline Data Analysis & $61.10 \%$ \\
\hline Local Analysis & $92.27 \%$ \\
\hline \multicolumn{2}{|c|}{ Percent Model } \\
\hline Stripping the Frontier & $65.14 \%$ \\
\hline Data Analysis & $76.86 \%$ \\
\hline Local Analysis & $85.55 \%$ \\
\hline
\end{tabular}




\section{Chapter 5: Discussion of Results}

This chapter presents a discussion of the results obtained for each model introduced in Chapter 4. The results, including efficiency scores, peer references and target setting, along with the applied methodologies are discussed separately for each model. The models are then be compared and the most advantageous models are identified.

\subsection{0-2011 PERIOD GROWTH MODEL}

This section discusses the results of the "2010-2011 Period Growth" model and assesses its usefulness in examining the growth potential of the Bank's branches.

\subsubsection{EFFICIENCY SCORES}

DEA provides overall technical efficiency scores as a means of measuring performance. For the output oriented models used in this study, the technical efficiency indicates the proportional increase of outputs that could be attained by using the same amount of inputs as other branches operating on the efficient frontier. The DEA scores of inefficient units are obtained through comparing the original unit with its projection onto the efficient frontier.

The efficiency scores obtained for the 2010-2011 Period Growth model were fairly consistent with the scores that are generally obtained from DEA branch analyses. This is because, under the same regulation and company culture, branches tend to operate similarly; thus, their relative efficiency scores tend not to deviate too much from one another.

The percent of efficient units resulting from each execution of the model is much lower than the common $25-40 \%$ observed in typical branch analyses. The traditional production, profitability and intermediation models focus more on the types of branch operations, which are relatively consistent between branches. Growth, on the other hand, is more variable between branches and could rely heavily on market conditions and saturation, the branches reputation in the community, or the ability to access new clientele. It follows that few branches may be operating under ideal conditions with a readily available pool of new clients. Examining the conditions and characteristics of the efficient units which were referenced by a large number of inefficient DMUs could provide further insight as to why they performed better than their peers and what other variables may be pertinent for inclusion in future analyses. 


\subsubsection{SEgMENTATION RESULTS}

Segmenting the DMUs' DEA results by market and size provided some insight into the operating characteristics of branches. When the 'existing customer base', 'funds managed' and 'urban/rural' were examined, it was evident that the Bank's smaller branches performed better under CRS assumptions while the larger branches performed more favorably under VRS assumptions. This may be attributed to larger branches having more variability in their operations and customers banking requirements, whereas small banks focus more on day-to-day banking and less complex products.

When segmented by market, each region performed similarly to the others. The fareastern and western provinces produced the lowest average efficiency scores for the CCR model. This may be attributed to large differences in culture between the coastal and mainland provinces. The GTA also performed poorly in the CCR model, which may be due to a greater number of large branches. The BCC model produced more uniform results for each market segment.

\subsubsection{PeER RefERENCES AND TARget SETTING}

Aside from identifying best practice units, DEA offers peer referencing and target setting capabilities, an advantageous managerial tool. For each inefficient unit, DEA provides a set of peer references whose linear combination is equal to the projection of the inefficient unit onto the frontier. Some inefficient units will have multiple peers while others may only have one.

Efficient units that are referenced a large number of times are considered to be doing better than other units and are used to create best practice guidelines for other units to emulate. However, highly referenced units may also be outliers operating in more favorable environments or with significant business advantages. Efficient units that are only referenced once or not at all are considered weakly efficient and may only be efficient because there is no one else to reference them against.

The top ten reference peers for the final runs of the CCR and BCC models were all referenced more than 100 times. Due to the larger number of efficient units, the units were referenced fewer times in the BCC model. DMU 745 was the only unit that fell into both the CCR's and BCC's top ten, with 927 CCR references and 446 BCC references. DMU 745 vastly outperformed the average branch growth of $4 \%$, with a mean growth of $35 \%$. Upon further investigation, it was determined that this branch opened in 2010 in Calgary, Alberta and held more loan accounts than deposit accounts, suggesting that it may be a real estate branch. 
Similarly, DMU 618, appearing in the top 15 of both models, shows similar characteristics of having a very large number of loan accounts as compared to deposit accounts. These findings infer that further branch segmentation is required to ensure that speciality branches are not included in the models.

The projection of an inefficient unit onto the frontier also provides realistic targets that can be used to formulate attainable goals for the unit. This too is a valuable managerial tool that allows managers to better assess the performance of the Bank's branches as well as the overall branch network performance. Figures 5.1 and 5.2 depict the average target setting objectives of the final run CCR and BCC models, respectively. The targets for the remaining CCR and BCC models are included in Appendix 3.

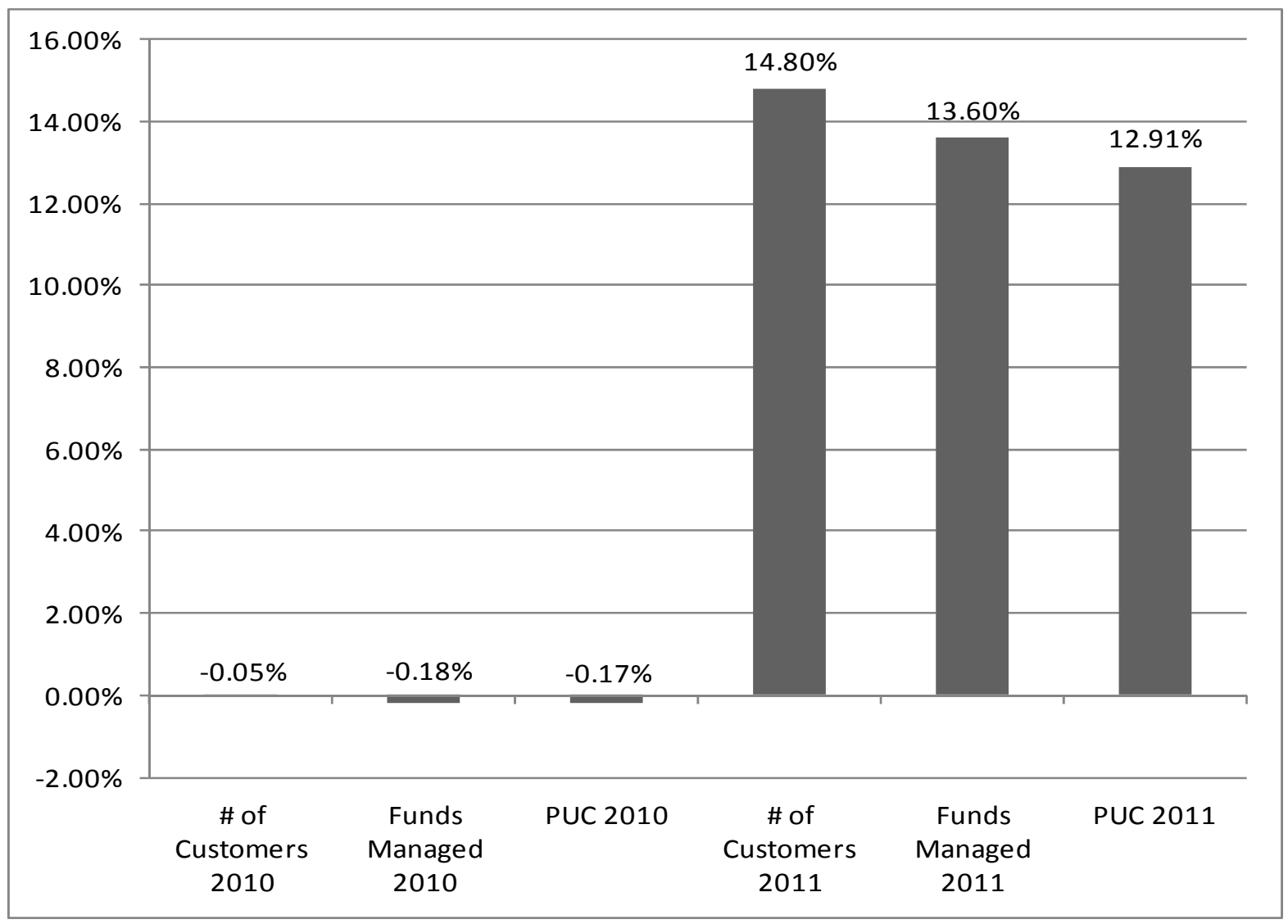

Figure 5.1: 2010-2011 Period Growth CCR Model Target Setting 


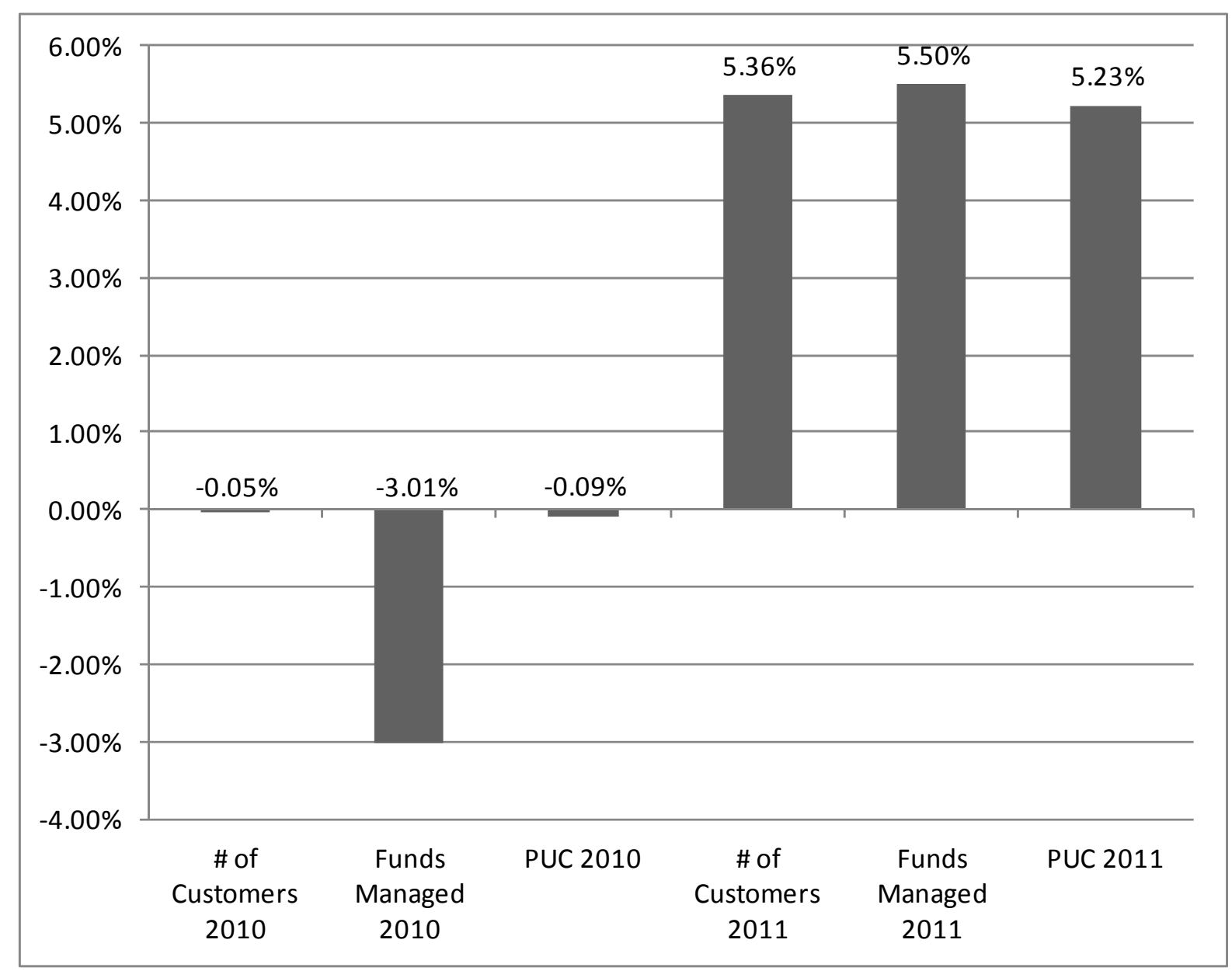

Figure 5.2: 2010-2011 Period Growth BCC Model Target Setting

Through comparison of the two graphs, it can be seen that the BCC model produces more attainable targets, with the maximum improvement being $5.5 \%$. The CCR model provides less realistic annual targets, with the maximum being $14.8 \%$.

\subsubsection{ASSESSMENT OF OUTLIER REMOVAL}

To determine whether stripping the efficient frontier was a suitable method of outlier removal, the average efficiency scores of each consecutive run, outlined in Table 4.5, were compared. For the CCR model, the average efficiency improved $15 \%$ between the first and seconds runs, $5 \%$ between the second and third run, and $7 \%$ between the third and final runs. The number of efficient units nearly doubled between each set of runs, the standard deviation consistently decreased and the minimum DEA score increased 32\% between the first to final runs. The efficiency distributions provided in Appendix 1 also depict the movement of the median from a fairly low efficiency score to a more typical value. 
This consistent improvement suggests that the method was successful in removing the outliers that dominated the frontier. The large changes observed between the first and second runs may infer that one strip of the frontier is sufficient for this model. However, having only removed 33 of the 1041DMUs for the final run, it was still advantageous to perform all three strips. It should be noted that not all of the stripped units are necessarily outliers, and should each be considered carefully by the Bank's management. Moreover, combining this methodology with more in depth knowledge of the Bank's branches and their specific operations could potentially produce more favourable results.

\subsubsection{SENSITIVITY ANALYSIS}

The sensitivity analysis performed for the "2010-2011 Period Growth" model provided further insight into the relevance of each variable to the model. As was shown in Table 4.6, the scores obtained after the removal of each variable from the CCR model differed only slightly from those obtained from the original CCR model. The results of the BCC model (Table 4.7) showed an even smaller difference between the original model and the modified models. In both models, it appears that the input variable 'number of customers' and the output variable 'product use count' had the largest impact on the DEA scores. Similar to the 2010-2011 Period Growth Model's correlation analysis, this analysis conceded that one or more variables could be removed from the model without significantly affecting the results. As previously mentioned, however, all of the variables were left in to provide a clearer objective to the Bank's managerial staff.

The results of the Rank-Sum test (Tables 4.8 and 4.9) were much more informative than the results of the efficiency contribution analysis. It identified several variables in both the CCR and BCC model that have a significant impact on the mean scores of the DMUs. This analysis suggests that 'PUC 2010', 'Funds Managed 2011' and' number of Customers 2011' greatly impact the DEA scores and should therefore not be removed from the CCR model. Similarly, 'PUC 2010 and 2011' and 'number of Customers 2010 and 2011' greatly impact the results of the BCC model, and thus should not be removed. If, at a later date, the Bank decides to reduce the number of input and output variables in these models, it should only consider removing variables that did not impact the mean scores. If multiple variables are removed, further sensitivity analysis should be performed. Moreover, the above sensitivity analyses were performed using the complete data set of 1041 DMUs, and thus may have been susceptible to the presence of outliers. 


\subsubsection{Overall Assessment of THE MOdel}

Overall, the 2010-2011 Period Growth model showed promise for future analysis. It produced realistic results and demonstrated good discriminatory power. Further segmentation should be performed along with local analyses and more focused removal of specialty branches. Moreover, as it stands, this model only assesses half of the "branch growth" picture; focusing solely on the products or "outputs" of the branch. To get a more complete picture of branch growth, a second model focusing on the resources or "inputs" used by the branch should be constructed. This dual model would allow the assessment of growth but would also consider how efficiently resources were used to obtain that growth.

\subsection{Lost Accounts/Gained Accounts Model}

This section discusses the results of the "2010-2011 Period Growth" model and assesses its usefulness in examining the growth potential of the Bank's branches.

\subsubsection{EFFICIENCY SCORES}

The efficiency scores and percent of efficient units obtained for the "Lost Accounts/Gained Accounts" Model were both lower than those generally obtained from traditional DEA branch analyses. As was stated before, this is most likely due to the unique nature of this model.

The efficiency distributions (Appendix 1) obtained from this model closely resembled normal distributions and had a broader range than those obtained for the 2010-2011 Period Growth model. The difference between the minimum observed efficiency and the average efficiency was consistently in the range of $30 \%$. This could indicate one of several things: the model has very good discriminatory power, the data set includes a large number of outliers, or global analysis is not appropriate and local analyses should be performed instead. To test these hypotheses, local analyses should be run and the results compared to those of the global analysis. Further discussion with branch management will also be required.

\subsubsection{SEgMENTATION RESULTS}

When the 'existing customer base', 'funds managed' and 'urban/rural' were examined, it was evident that the Bank's smaller branches performed better under CRS assumptions while the larger branches performed more favorably under VRS assumptions. This may be attributed to larger branches having more variability in their operations and customers' banking requirements, 
whereas small branches focus more on day-to-day banking and less complex products. When segmented by market, each region performed similarly to the others in both the CCR and BCC models and no trends were apparent.

\subsubsection{PeER REFERENCES AND TARget SETting}

The top ten reference peers for the final runs of the CCR and BCC models were all referenced more than 100 times and three units were found in both the top ten reference sets (DMUs 983, 912 and 633). These branches held very few similarities; branch 633 was an average sized urban branch, 912 was a large branch with a high number of loans accounts compared to deposits and 983 was a rural branch that held approximately $25 \%$ market share. Upon further investigation, it was determined that branches 633 and 983 demonstrated customer retention that was $2 \%$ higher than average, and customer attrition that was $20 \%$ lower than average. They were both on par with the average for attracting new customers. Conversely, branch 912 performed less well than average in both customer retention and attrition, but performed three times better than average in attracting new clients. Paired with the high number of loan accounts and low number of other account types, this tends to suggest that this branch may be more real-estate focused than the average branch.

The average target setting objectives for the final run CCR and BCC models are provided in Figures 5.3 and 5.4, respectively. The targets for the remaining CCR and BCC models are included in Appendix 3.

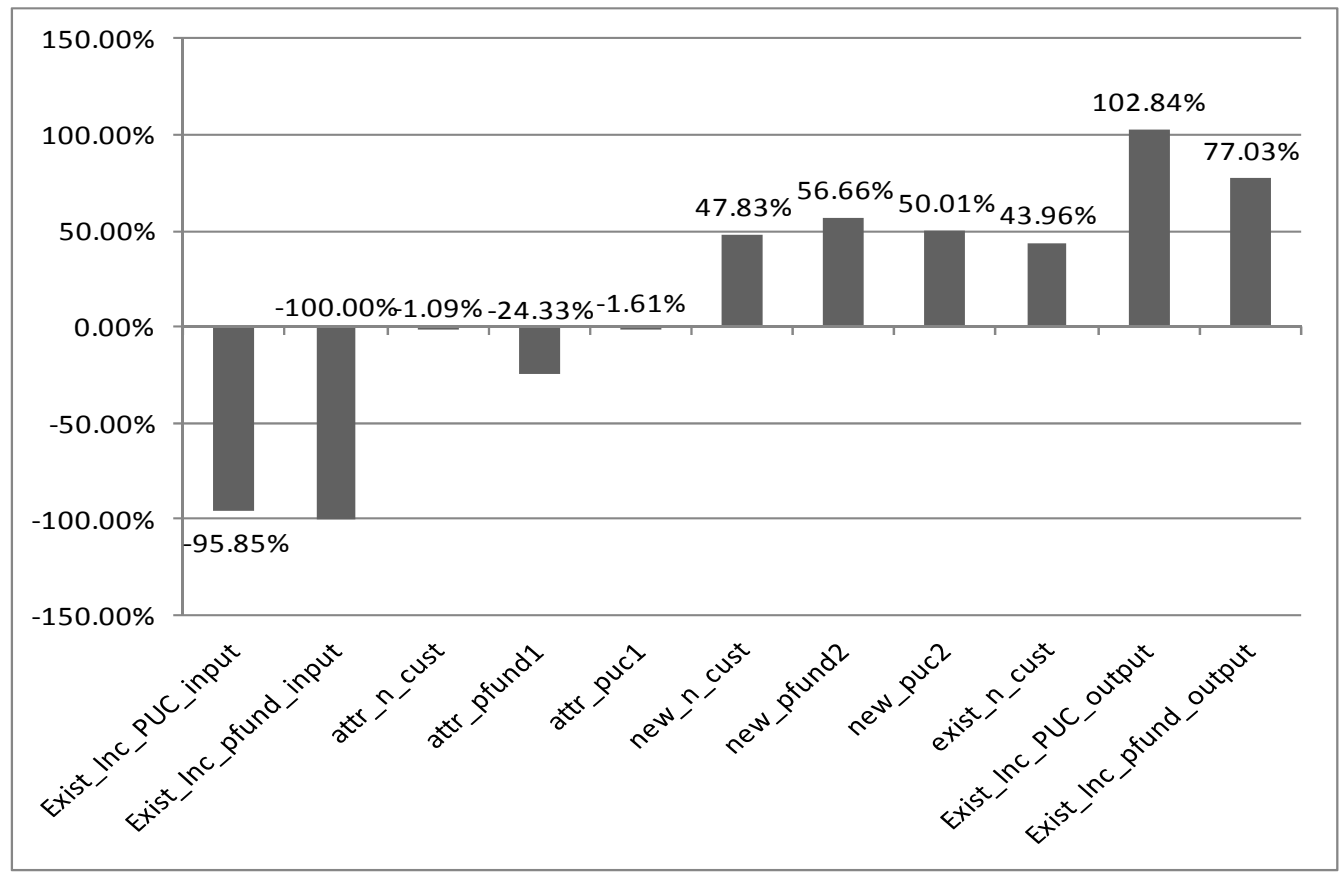

Figure 5.3: Lost Accounts/Gained Accounts CCR Model Target Setting 


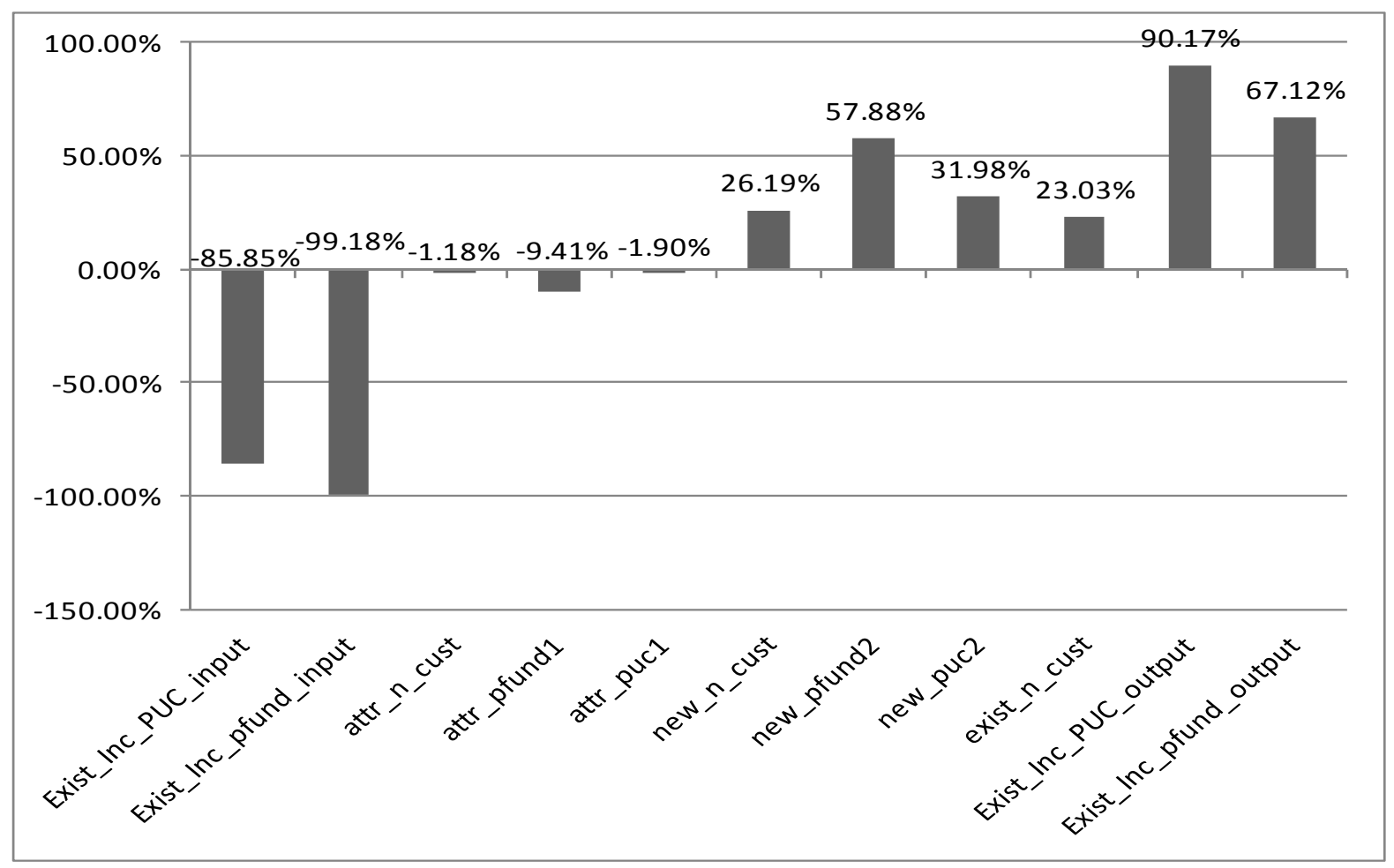

Figure 5.4: Lost Accounts/Gained Accounts BCC Model Target Setting

The target projections provided by the Lost Accounts/Gained Accounts models are very high and rather unrealistic annual goals. It is apparent that multiplier restrictions should assign limits so that more useful target setting is provided. Although the results are in some cases impractical, DEA was still able to assign peers and provide a direction for each branch to work towards.

\subsubsection{ASSESSMENT OF OUTLIER REMOVAL}

To assess the effectiveness of stripping the efficient frontier for the Lost Accounts/Gained Accounts models, the differences in the results between each model run were examined. Referring back to Table 4.11, the average efficiency and minimum efficiencies increased by approximately $7 \%$ between each run and the percent of efficient units increased by 1-2\%. Compared to the "2010-2011 Period Growth" model, the changes seen here are quite small. Also, their uniform nature may suggest that this method doesn't target outliers efficiently, or that outliers have less of an overall affect on this model. Although these findings do not invalidate the use of this method for this model, they also do not heavily support the use of this methodology.

The efficiency distributions, provided in Appendix 1, are all positively skewed but become less skewed with each strip of the frontier. This may infer that the dominant outliers are 
slowly being removed. However, it may also be the case that this model has simply identified that the majority of DMUs are relatively inefficient at retaining and attracting customers. In order to determine for certain which is the case, more managerial insight and specific branch information is required.

\subsubsection{Overall Assessment of Model}

The Lost Accounts/Gained Accounts model provided good discriminatory power and was able to clearly identify units that outperformed others in the areas of customer retention, and new customer gains. However, there were apparent limitations in its ability to provide realistic targets. If used for future analysis, multiplier restrictions should be imposed to provide more realistic targets and outliers and specialty branches should be more carefully removed. This model would also benefit from local analysis, which would likely reduce the positive skew that currently exists in the efficiency distributions.

Aside from the above issues, the Lost Accounts/Gained Accounts model offers a unique perspective that provides a lot of insight into which branches are better at retaining customers and attracting new clients. From here, the Bank may be able to gain a better understanding as to why clients stay or leave and which branch characteristics result in better retention of customers. Moreover, this model can help identify which marketing strategies and delivery methods are attracting new customers. All in all, with more branch-related information and managerial insight, this model could be of great use to the Bank.

\subsection{MARKET MODEL}

This section will discuss the results of both Market models and will assess their usefulness in examining the market efficiency of the Bank's branches. The section concludes with a comparison of the two models and the identification of which is most appropriate for further analysis.

\subsubsection{COMPARISON WITH OTHER STUDIES}

Direct comparison between DEA results from other bank branch efficiency studies is not possible since DEA produces relative efficiency scores. However, general comparison can be performed to help identify trends and to assess the validity of the model results. Although the majority of the models contained within this study take unique approaches not yet found in literature, market models, similar to the one presented in this study, have been published along 
with their results. This offers the opportunity to compare the results of the "Market" model to those found in literature.

Amongst the identified bank branch studies, Athanassoupoulos' [ATHA98] was the only market model DEA analysis that provided actual DEA scores. This study, consisting of 580 DMUs produced average efficiency scores ranging from 0.84 to 0.93 , which is similar to the range of 0.88 to 0.94 obtained in this study. Moreover, his study showed that larger branches, whether segmented by assets or size, performed better than smaller branches, which correlates to the BCC model used in this study.

\subsubsection{EFFICIENCY SCORES}

The efficiency scores obtained from the Market models fell within the general range identified by literature. However, when comparing the scores of the Component and Aggregate models, it is apparent that the values of both the efficiency scores and percent efficiency are much higher for the Component model than for the Aggregate model. The Component model returned average efficiency scores that ranged from 0.92 to 0.99 and produced $55-91 \%$ efficient units. These values indicate that the discriminatory power of the model is very poor and is not able to effectively identify true best performers. On the other hand, the Aggregate model offered higher discriminatory power with an efficiency range of 0.87 to 0.96 and anywhere from $30 \%$ to $75 \%$ efficient units.

Examination of the efficiency distributions provided in Appendix 1 show that the Component model distributions are heavily dominated by efficient units. This agrees with the above hypothesis that this model does not offer adequate discrimination between units. Although the Aggregate model distributions also show a high number of efficient units, the units are fairly evenly distributed.

\subsubsection{SEGMENTATION RESULTS}

Both Market models offered the same conclusions when their results were segmented by customers, funds and urban/rural. Under both CRS and VRS conditions, the rural branches outperformed the urban branches. When segmented by funds and customers, the XL branches closely followed by small branches outperformed L and M branches. This may imply that small branches and very large branches performed better in their given market conditions. However, the segment for the XL branches consists of very few units which may lead to an unfair comparison with the other segments which consist of far more units. When split by market, there 
were only slight differences between each region, with none performing substantially worse than its counterparts and no observable trends.

\subsubsection{RETURNS TO SCALE}

Upon examination of the RTS distributions and the scale efficiencies of the market models, it was apparent that since the scale efficiencies were so high (95\%-99\%) the difference between the DRS units and the CRS units must be minimal. For clarity, a two dimensional depiction of the CCR and BCC frontiers is provided in Figure 5.5. As shown, the DRS section of the BCC frontier is very closely situated to the CCR frontier. If a "thick frontier" were used, it is probable that a large portion of the DRS units would then fall under CRS. As banking operations are heavily regulated and branch operations are relatively consistent within the Bank, the conclusion that the majority of the branches fall under or are very close to CRS is realistic.

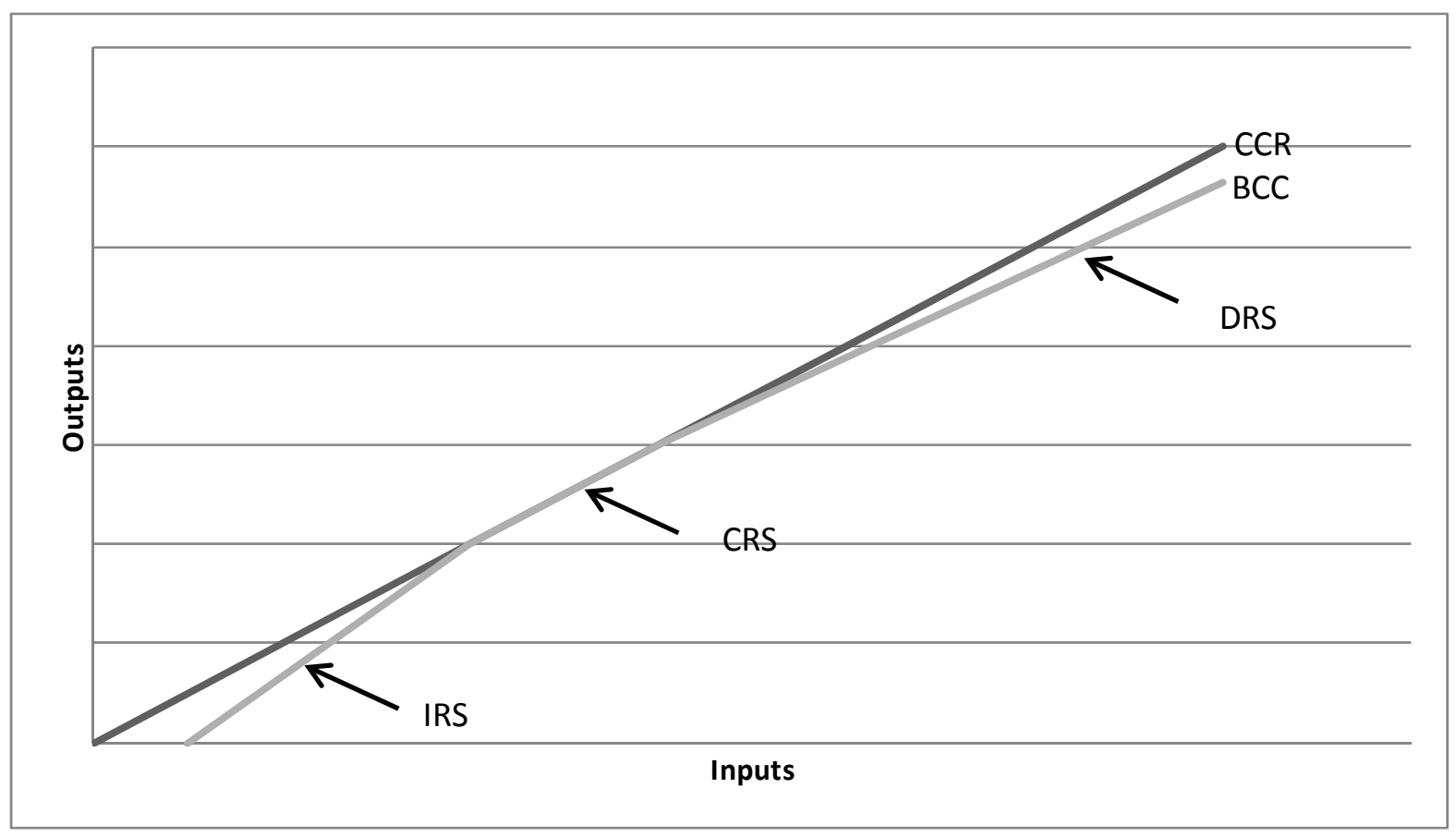

Figure 5.5: Two-Dimensional Representation of CCR and BCC Frontiers

\subsubsection{PEer REFERENCES AND TARget SETting}

Since such a large number of efficient units were stripped for the final runs of the CCR and $\mathrm{BCC}$ models, this section focuses on the peer referencing and target setting for the second run values.

\subsubsection{COMPONENT MODEL}


With such a large number of efficient units, the efficient peers were referenced far fewer times than those from the previous models. The top ten CCR references were referenced an average of 102 times and 64 times for the BCC top references. Of the top units for each model, seven units were found in both the CCR and BCC set. These units are all found in central or eastern Canada and are evenly divided among rural and urban and appear to be a mix of large, medium and small branches.

The average target setting objective for the Component models are provided in Figures 5.5 and 5.6. The target projections for the CCR model are quite high with the majority over $20 \%$. Those of the BCC model are somewhat more realistic, ranging between 5 and $15 \%$. In order to produce more usable results, multiplier restrictions should be incorporated into the model. For example, an increase in market share of $20 \%$ is very unrealistic and most likely impossible to attain under normal circumstances.

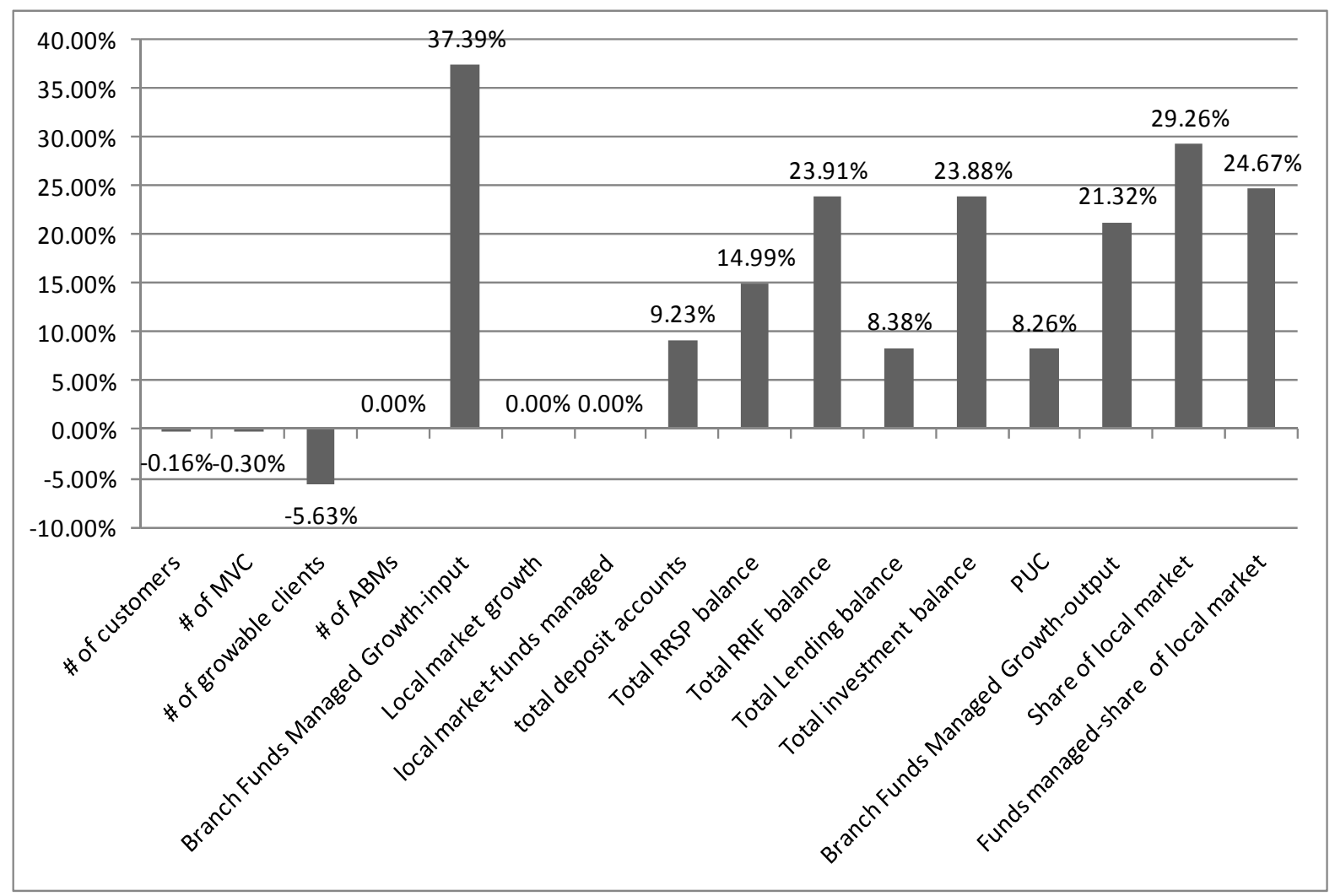

Figure 5.6: CCR Component Model Target setting 


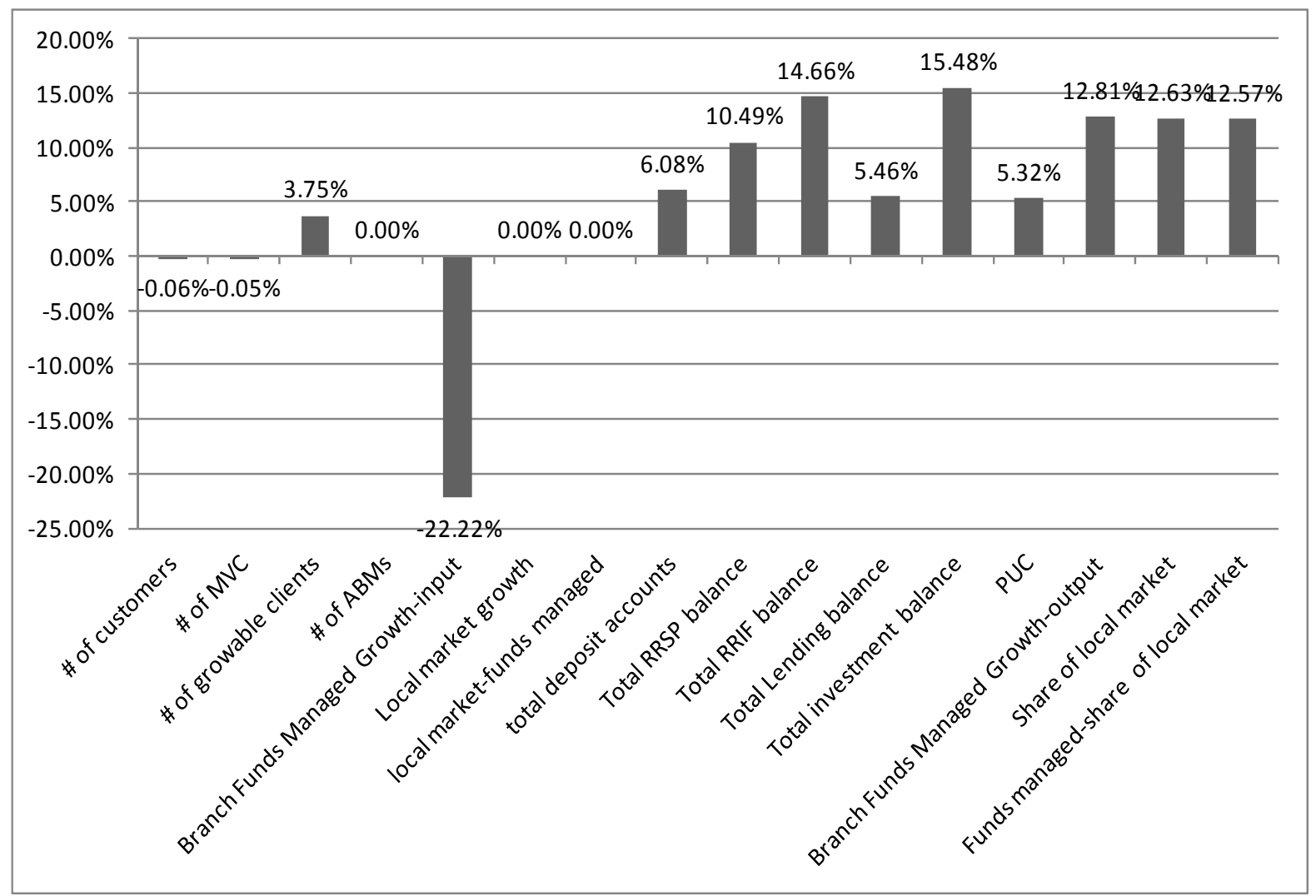

Figure 5.7: BCC Component Model Target Setting

\subsubsection{AGgregAte Model}

Having less efficient units than the Component model, the top ten referenced units of the CCR and BCC Aggregate models were all referenced more than 100 times. From the ten CCR and BCC units, six units were present in both sets. Like those of the component model, these units were situated in either Central or Eastern Canada and varied in region and size.

The target setting objectives provided by the Aggregate model, shown in Figures 5.7 and 5.8, are quite high. It follows that this model too should be constricted with multiplier restrictions. 


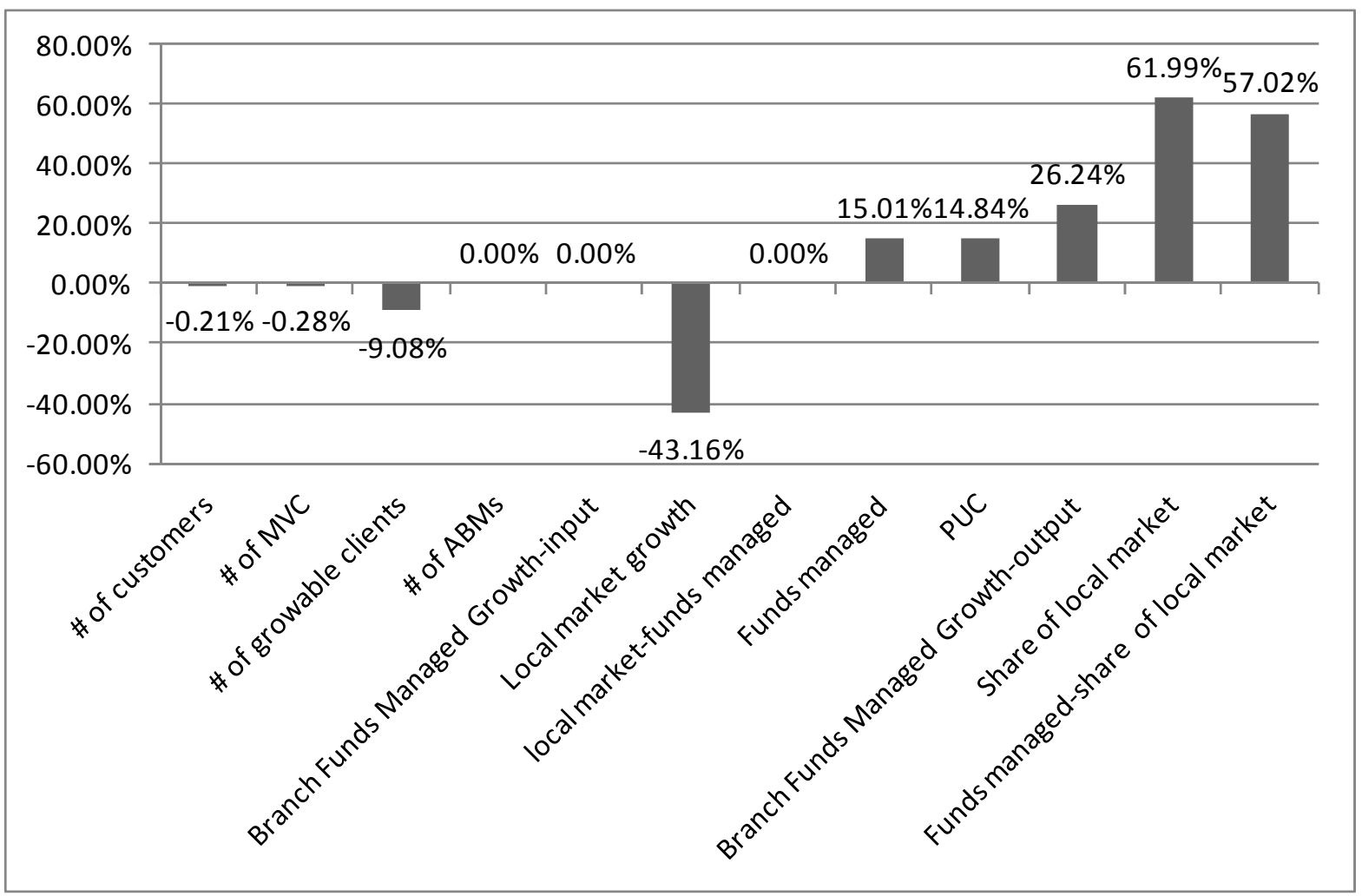

Figure 5.8: CCR Aggregate Model Target setting

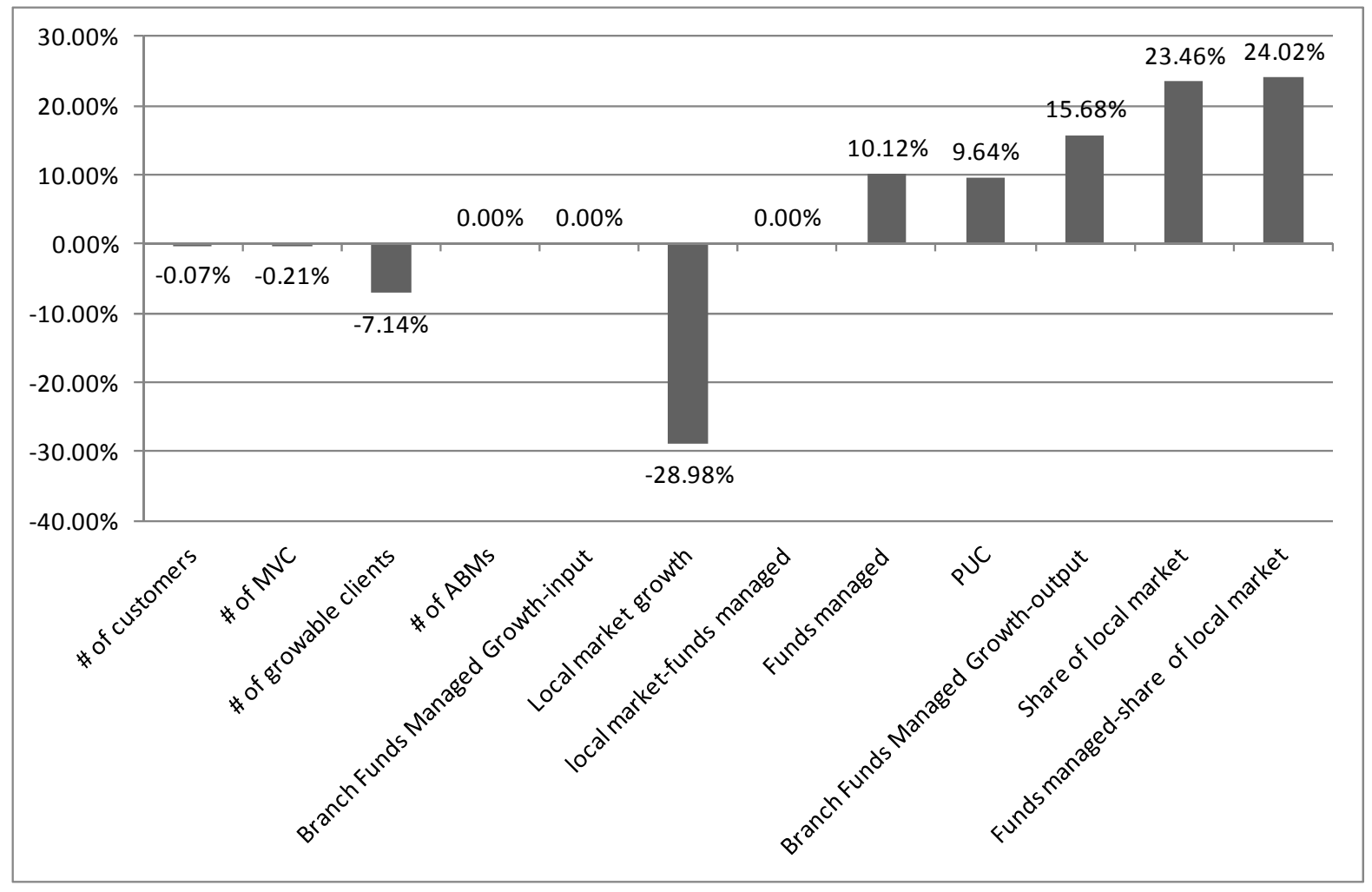

Figure 5.9: BCC Aggregate Model Target Setting 


\subsubsection{Assessment Of APPlied Methodology}

As depicted in Tables 4.14 and 4.15, stripping the efficient units between runs two and three did improve the efficiency score. However, since such a large number of units were identified as efficient, many units that are not outliers are stripped in the process removing a large portion of the pertinent DMUs. Moreover, the observed change is relatively small and does not have a significant effect on the outcome. It follows that that stripping the efficient frontier is not ideal for this model.

\subsubsection{Overall Assessment of Model}

With the availability of market related branch data, the Market model offered the opportunity to assess the Bank branches' relative market efficiency given their unique market conditions. Through analysis of the results obtained from these models, it is apparent that multiplier restrictions are needed and other outlier removal techniques should be investigated. For these two processes to be successful, more branch specific data and managerial insight is required.

It was also determined that the discriminatory power of the Component model was quite poor and the model did not produce meaningful results. The Aggregate model offered better discrimination; however, if pursued, careful managerial considerations are required. Paired with a model that focuses on internal branch to branch comparison, a more complete picture of branch growth could be obtained.

\subsection{Delta ModeL}

This section discusses each form of the Delta model along with the effectiveness of the applied methodologies. The competencies of the models in attaining their objective are assessed and their functionality determined.

\subsubsection{EFFICIENCY SCORES}

When the stripping technique was used in conjunction with the Delta models, low average efficiency scores were obtained and the efficiency distributions were heavily skewed in the positive direction. This suggests that the models were dominated by outliers or noncomparable units. The models also resulted in a very low number of efficiency units.

The results from the removal of DMUs were somewhat improved; however, the distributions were still heavily skewed and the number of efficient units were very low. When 
local analysis was used, the efficiency scores of both models increased drastically, ranging between 0.75 to 0.85 . The resulting distributions were also much less skewed and more closely resembled normal distributions

Upon examining the efficiency scores and distributions of the separate models and methodologies, it is apparent that these models have some inherent limitations. There were several small clusters of DMUs that dominated the data set and inhibited the production of any usable results. Moreover, DEA attempts to make each unit as efficient as possible by minimizing where a unit performed poorly and maximizing where they performed well. Without multiplier restrictions, DEA will assign a zero (or near zero) values for poor performance, which, in turn, can heavily skew the results.

\subsubsection{Segmentation Results}

When the results of the models were segmented, it was consistently found that urban branches outperformed rural branches. This may be attributed to the fact that rural branches generally have more stagnant local markets and client bases while urban branches are subject to constantly changing local environments and population growth rates. When segmented by funds and customers, it was also observed that the larger branches outperformed the smaller branches. This held true for all models and methodologies with the exception of the Percent model when the efficient frontier was stripped. In this case, the small branches outperformed the large branches. This contradiction to the other models' results is likely due to the use of percentages. When segmented by region, the results were inconclusive, with each model identifying different best and worst performing regions.

\subsubsection{Peer References and Target Setting}

For brevity, the peer reference and target setting results for these models are discussed as a whole. The associated target setting graphs can all be found in Appendix 3. In general, the target setting objectives obtained from the Delta models were very high and largely unattainable. When the outliers were removed through stripping, the targets ranged from $30-400 \%$. When statistical data analysis was used, the targets ranged between $85 \%$ and $495 \%$; and when local analysis was used, the targets ranged between $4 \%$ and $77 \%$. It is obvious that these targets are highly unrealistic and offer little usable advice to the Bank.

To improve the results of this study, multiplier restrictions must be used. This would most likely result in a drastic change of results and may lead to a more informative model. As it 
stands, these models offer little in the way of proper identification of best performers or realistic target setting.

\subsubsection{Assessment OF APPLIEd Methodology}

Unlike the previously discussed models, the Delta models were subject to numerous analysis techniques. This section discusses the success of each of the applied methodologies.

\subsubsection{STRIPPING EFFICIENT FRONTIER}

Although stripping did in fact result in an increase in average efficiency and number of efficient units, the observed changes between each strip were very small. Upon investigating the efficiency distributions it could be seen that there were a large number of small DMU clusters which dominated the higher efficiency scores. In conclusion, the stripping technique was particularly effective in removing outliers.

\subsubsection{STATISTICAL DATA ANALYSIS}

Removal of DMUs based on statistical analysis appeared to provide some increases in the average efficiency scores; however, the results of the analyses were still inconclusive when this methodology was used.

\subsubsection{LOCAL DEA}

In the initial analysis of the Delta model a small number of units dominated the efficient frontier, resulting in very low average efficiency scores. In order to better assess the efficiency of the DMUs, and to provide more realistic peer references and target setting a local analysis was performed. This offers the opportunity to compare units to those that are most comparable. It follows that the increase in DEA scores resulting from local analysis can be attributed to the decrease in differentiation between compared DMUs. This section discusses the segments used for the local analyses and examines the obtained results.

\section{Difference Model}

When local analysis was performed on the Difference model, the average efficiency scores increased $25.1 \%$ for the CCR model and 30.1\% for the SBM model. This substantial increase is due to the fact that DMUs were being compared only to like units. Although there was an increase in the number of efficient units, it was relatively small compared to the increase in average DEA score. Moreover, the peeling of the efficient frontier provided little benefit, 
increasing the average efficiency by $1 \%$ for the CCR model and $2 \%$ for the SBM model. This suggests that the outliers have little effect when local analysis is used as they too are grouped only with their most comparable units.

\section{Percent Model}

Similar results were obtained when local analysis was performed on the Percent model. The average efficiency scores increased $22.6 \%$ for the CCR model and $30.7 \%$ for the SBM model. Again, little difference was noted when the first run of the local analysis was stripped of its efficient frontier. The CCR model's average efficiency increased by 1\% and the SBM model's by $2 \%$.

Compared to the other methodologies used for these models, the local analysis produced the best results. By only comparing units with their most similar peers, the models were no longer dominated by large units and outliers. However, the targets obtained from local analysis were still quite unrealistic and further modification to the models would still be required to produce usable and significant results.

\subsubsection{OVERALl Assessment OF MOdel}

As implied in the previous sections, the Delta models provided few usable results and were unable to properly differentiate best performers. Their efficiency distributions were heavily skewed, suggesting poor DMU classification and their target setting objectives were unrealistic. The presence of dominant outliers and the inherent limitations of DEA resulted in inconclusive results. Moreover, there were several issues associated with the data itself that may have led to these poor results. The data set included ratios, required translations and incorporated a dummy variable all, of which may have affected the effectiveness of the models.

In closing, it is advisable that these models not be pursued further as they did not meet the desired objective and illustrated that they each had inherent weaknesses that rendered incongruous results.

\subsection{MODEL COMPARISON}

Through analysis and consideration, it has been determined that some of the above models are not suitable for use in the real world while others deserve further investigation. Firstly, the "2010-2011 Period Growth" model offers a very unique opportunity to use DEA to assess the changes in a branch over time. With more data and managerial insight, this model has 
significant potential and should be pursued further. The "Lost Accounts/Gained Accounts" model also took a unique perspective in assessing the ability of a branch to retain and attract customers. It had good discriminatory power and was able to properly identify best performing units. However, the results showed a definite need for multiplier restrictions which should be addressed. Given realistic restrictions, this model should also be pursued for future work.

The Market models showed some definite limitations in their ability to differentiate efficient and inefficient units. They also provided targets that were largely unattainable within a sensible number of years. That being said, the Component Market model does not produce significant results and should not be considered further for this study. With additional insight from management, and variable aggregation or reduction, the Aggregate Market model may still provide useful information. Moreover, employing the Aggregate model in conjunction with the "2010-2011 Period Growth" model or the "Lost Accounts/Gained Accounts" model will allow for a more complete picture of a branch's growth potential.

Lastly, the Delta models had inherent limitations that made them unsuitable candidates for further analysis. Their incorporation of ratios and use of translations are not ideal and result in the loss of meaning. The obtained efficiency scores were also very low and their distributions very wide suggesting that the models are heavily susceptible to outliers. Although improved results were obtained with the application of different methodologies, the models, as a whole, did not succeed in meeting their objectives or in providing significant results.

Overall, this analysis was successful in identifying models that provide usable results pertinent to understanding branch growth. The "2010-2011 Period Growth" model, the "Lost Accounts/Gained Accounts" model and the "Aggregate Market" model each produced informative preliminary results that will only improve with further managerial insight. It should also be stated that there is an outstanding opportunity using these approaches to make a significant contribution to branch network' growth and profitability if more data was available and multiplier constraints are designed. 


\section{Chapter 6: \\ Conclusions and Recommendations}

This chapter presents a brief synopsis of the research, along with the conclusions drawn from the examination and analysis of the results. Directions for future work follows.

\subsection{CONCLUSIONS AND RECOMMENDATIONS}

This study involved the development and analysis of four different DEA modeling approaches that examined the growth potential of the branches of a major Canadian Bank. The goal of the study was to better identify growth potential and best performers within the branch network. Having received a rather substantial data set from the Bank, the study provided the unique opportunity to assess the functionality of several models with differing perspectives.

In total, four model objectives were defined and six models were constructed and analyzed using varying methodologies. The "2010-2011 Period Growth" model assessed the ability of branches to grow products sold, funds managed and new customers from the year 2010 to 2011. It was found to be successful in identifying best performers and offered good discriminatory power. Moreover, combining this model with its "input" counterpart will allow for a more complete picture of banking operations.

The Lost Accounts/Gained Accounts model provided a good basis for future work. It was able to successfully identify best performers and offered strong discriminatory power. However, it did provide unrealistic targets, hence, some modifications, including the use of cone ratios or multiplier weight restrictions, should be considered in the future. In any case, the Lost Accounts/Gained Accounts model offers a unique perspective that provides insight into which branches are best at retaining and attracting clients.

The modeling approaches were taken to assess the market efficiency of the Bank's branches; the component model approach which used all branch products as separate outputs and the aggregate model that combined all of the products into one metric. The Component model provided poor discriminatory power and offered little in terms of conclusive results. The Aggregate model showed higher discriminatory power, but would still benefit from further variable reduction. It is comforting to note that the Aggregate model produced very similar results to those found in literature. With the use of multiplier restrictions and managerial insight, 
the Aggregate model could be valuable in understanding branch growth relative to local market conditions and should be pursued further.

The Delta models were found to be largely unsuccessful in identifying best performers or in providing realistic target setting objectives. Their inherent limitations, use of ratios and lack of input data have together resulted in ineffective models. Although, the use of local analysis did improve the results, the overall performance of the models was subpar as compared to the other models introduced in this study. In consequence, these models should not be pursued for future analysis unless more detailed and accurate data becomes available.

\subsection{DIRECTIONS FOR FUTURE WORK}

As compared to more traditional DEA bank branch analyses (i.e. production, intermediation and profitability), the study of branch growth using DEA is a fairly untouched field of research. It, therefore, has great research potential and offers plenty of new and exciting opportunities. Those more closely related to this study include the use of cone ratios, local analysis and accounting for environment factors. Moreover, performing the above analyses over a longer time frame or using customer specific data would be interesting and informative extensions.

\subsubsection{Restricted Multipliers (CONE RATIOS)}

Without multiplier restrictions, the results obtained from a DEA analysis may not be realistic. Restricted multipliers are often used to produce more realistic target setting results and to better represent managerial decision making. Moreover, they influence the relative importance of each variable as seen by management. Determining the appropriate range for each multiplier requires accurate managerial insight and information about bank specific operating restrictions.

The results of the models discussed in this study could be greatly improved with the use of multiplier restrictions. It would allow for more attainable target setting and would provide a more realistic representation of the Bank's branch performance. If pursued, this will require managerial input to ensure that real life issues are properly accounted for.

\subsubsection{LOCAL ANALysis}

As shown for the Delta model, local analyses can produce very desirable results. Since there was such a large spread of branch types and sizes, and operating environments within the data set, local analysis may allow for a more accurate representation of individual branch 
efficiencies. Information obtained from this study may help to better identify best performers and appropriate peer references.

\subsubsection{ENVIRONMENTAL FACTORS}

Operating environment can play a significant role in determining how efficiently a bank branch operates. Although two branches may look comparable on paper, their environments may give one an advantage or disadvantage. To account for the cultural differences and better assess bank branch performance, a culturally adjusted DEA model, as introduced by Paradi et al. [PARA10], can be used. This methodology requires substantial information about the operating and corporate environments of each branch.

\subsubsection{EXTENDING THE 2010-2011 PERIOD GROWTH MODEL}

As was briefly mentioned, the 2010-2011 Period Growth model is only half of the complete branch growth picture. It effectively defines the 'outputs' of branch production but does not take into account the resources or 'inputs' that are used by the branch. To develop a more complete picture, an equivalent model using the resources used by the branch in 2011 as the inputs and the 2010 resources as the outputs should be constructed. The results of both models should then be combined using a second DEA model to provide an overall measure of branch efficiency.

The "2010-2011 Period Growth" model offered a very unique opportunity to assess branch efficiency over a period of time. This shed light on another possible extension where the analysis is extended over a longer period of time to provide a better understanding of the dynamics of branch growth. Combining DEA with machine learning techniques may also allow for the development of a predictive model. This would allow the Bank to better focus their resources and growth strategies.

\subsubsection{CUSTOMER DATA}

Bank branch growth is heavily based on the actions and movements of customers. Being able to better identify growable customers and understand the reasons behind customer attrition could provide the Bank with a significant advantage against their competitors. In order to do this, much more detailed customer data would have to be analysed and compared with overall branch performance. Data over extended periods of time (several years) would also be required in order to gain insight into the ebb and flow of customers within the branch network. Moreover, 
assessing how to properly match marketing strategies with specific client types may be valuable in increasing the number of retained and new clients.

This study would require an extensive set of data alongside managerial and employee insights into customer behaviours and marketing techniques. Furthermore, due to the limitations of DEA it would need to be combined with another suitable multiple linear objective programming technique to provide better predictive capabilities. 


\section{Glossary}

ABM

Additive Model

Allocative Efficiency

Bank Draft

BCC or VRS Model

Categorical Variable

CCR or CRS Model

Combined Model

Correlation

CRS

DEA

DFA

DMU
Automated Banking Machine; Self-service machines that allow customers of a financial institution to perform many everyday "banking" tasks, often at locations other than the institution.

A DEA model which measures efficiency using the slacks only. Involves simultaneous reduction in inputs and increase in outputs.

A measure of the ability to combine inputs and outputs in optimal proportions in the presence of market prices.

A cheque drawn by a bank against funds deposited in another bank.

A DEA model which assumes a variable return to scale relationship between inputs and outputs.

A variable that assigns a DMU to a specific class with predefined discrete values.

A DEA model which assumes a constant return to scale relationship between inputs and outputs.

A DEA model employing the results of three distinct DEA analyses as outputs and a fixed input of 1 .

A measure of the strength of the relationship between two coefficient variables. The value lies between +1 and -1 , with a score of 0 implying no relationship.

Constant Returns to Scale; A measure where a proportionate increase in inputs results in an identical proportionate increase in outputs.

Data Envelopment Analysis; A non-parametric, linear programming technique used to measure the relative efficiency of homogenous decision making units.

Distribution Free Approach; A parametric frontier approach to performance analysis.

Decision Making Unit; A term used to describe a unit being analyzed by DEA, in this case bank branches. 
DRS

Economies of Scale/

Returns to Scale

EFA

Efficiency

Efficient Frontier

FDH

FTE

GRS

Index Number

Input-Oriented Model A DEA model whose objective is to minimize inputs while

IRS

Market Efficiency

Market Model

Mix Inefficiency keeping outputs constant.

Decreasing Returns to Scale; A measure where a proportionate increase in inputs results in a less than proportionate increase in outputs.

The changes in output resulting from proportional changes in input.

Econometric Frontier Approach; Also referred to as Stochastic Frontier Approach (SFA).

A measure of the ability to produce outputs using a minimum level of inputs.

Empirical frontier that represents the "best performance" and consists of the decision making units in the data set that are most efficient at transforming inputs into outputs.

Free Disposal Hull; A non-parametric linear programming technique that constructs a stepwise efficient frontier.

Full-Time Equivalent; Total number of full time employees required to complete the task.

Generalized Returns-to-Scale denotes a BCC model whose convexity constraint is given a lower and upper bound.

A statistic that measures the change in weighted variables with respect to a standard or base.

Increasing Returns to Scale; A measure where a proportionate increase in inputs results in a more than proportionate increase in outputs.

The extent to which individual branches, given their capacity and available resources, utilize their market potential by maximizing sales.

A DEA model which captures a bank branch's ability to utilize their market potential to maximize sales using staffing, and market and branch characteristics as inputs and sales as outputs.

The non-radial inefficiencies, calculated by dividing the SBM efficiency score by the CCR efficiency score. 
MPSS

NDRS

NIRS

Non-Discretionary Variable

Output-Oriented Model

Overall Efficiency

Peer Group

Production Function

Production Model

Production Possibility Set

Productive Efficiency

Profitability

Efficiency

Profitability Model

PUC

Relative Efficiency
Most Productive Scale Size; The point on the efficiency frontier where the maximum average productivity is achieved for a given input/output mix.

Non-Decreasing Returns-to-Scale denotes a BCC model whose convexity constraint is $1 \leq \sum \lambda_{j}$ resulting in only constant or increasing returns-to-scale.

Non-Increasing Returns-to-Scale denotes a BCC model whose convexity constraint is $1 \geq \sum \lambda_{j}$ resulting in only constant or decreasing returns-to-scale.

A variable that is pertinent to the analysis but whose level of use or production is uncontrollable by management (i.e. weather).

A DEA model whose objective is to maximize outputs while keeping inputs constant.

The efficiency measured as a product of technical and allocative efficiency.

A set of efficient units to which the inefficient has been most directly compared within a DEA analysis.

The function in which outputs are defined as functions of inputs.

A DEA model that captures the business operations of a bank branch using staffing as inputs and transactions as outputs.

Given the observed data, the set of all possible input/output combinations that could exist.

The extent to which individual units, given their available resources, are able to produce outputs, in this case transactions.

The extent to which individual units are able to maximize revenues while minimizing expenses.

A DEA model that captures the business operations of a bank branch using revenues as outputs and branch expenses as inputs.

Product Use Count is a term used by the "Bank" to represent the number of products held by a client. The PUC of a branch is the sum of the branch's client PUCs.

The measure of actual performance of a unit relative to the best observed performance of other units in the set. 
SBM

Scale Efficiency

SFA

Share of Wallet
(SOW)

Slack Variable

Target

Technical Efficiency

TFA

Theoretical Frontier

VRS

Weight/Multipliers
Slacks Based Model.

Efficiency that indicates whether a unit is operating at its optimal size. This measure is calculated as a ratio of CCR to BCC efficiency.

Stochastic Frontier Approach; A parametric frontier approach to performance analysis.

The percentage (share) of a customer's financial products (wallet) that are with the firm in question.

Slacks represent the over-utilization of inputs or the underproduction of outputs in a DEA evaluation.

The value of inputs and outputs that would result in an inefficient unit becoming efficient.

The measure of the ability of a unit to produce the maximum output for a given set of inputs.

Thick Frontier Approach; A parametric frontier approach to performance analysis.

The frontier of best possible production that is not necessarily based on the performance of the observed units.

Variable Returns to Scale; A measure where a proportionate increase in inputs could result in a proportionate increase or decrease in outputs.

The coefficients applied to the input and output variables. 


\section{References}

[AIGN77] Aigner, D., Knox Lovell, C.A., Schmidt, P., "Formulation and estimation of stochastic frontier production function models", Journal of Econometrics, vol.6, pp.21-37, 1977

[ALTA07] Al-Tamimi, H.A.H., Lootah, A.M., "Evaluating the operational and profitability efficiency of a UAE-based commercial bank", Journal of Financial Services Marketing, vol.11, pp.333-348, 2007

[ATHA00] Athanassoupoulos, A.D., "The use of Data Envelopment Analysis in Banking Institutions: Evidence from the Commercial Bank of Greece", Interfaces, vol.30, pp. $81-95,2000$

[ATHA95] Athanassopoulos, A. D. "Performance Improvement Decision Aid Systems in Retail Organizations Using Data Envelopment Analysis", Journal of Productivity Analysis, vol.6, pp.153-70, 1995

[ATHA97] Athanassoupoulos, A.D. "Service Quality and Operating Efficiency Synergies for Management Control in the Provision of Financial Services: Evidence from Greek Bank Branches", European Journal of Operations Research, 98, pp.300-313, 1997

[ATHA98] Athanassopoulos, A.D., "Nonparametric frontier models for assessing the market and cost efficiency of large-scale bank branch networks", Journal of Money, Credit, and Banking, vol.30, pp.172-92, 1998

[BANK04] Banker, R.D., Cooper, W.W., Seiford, L.M., Thrall, R.M., Zhu, J., "Returns to scale in different DEA models", European Journal of Operational Research, vol.154, pp.345-362, 2004

[BANK84] Banker, R.D., Charnes, A., Cooper, W.W., "Some Models for Estimating Technical and Scale Inefficiencies in Data Envelopment Analysis", Management Science, vol.30, no.9, pp.1078-1092, 1984

[BANK86] Banker, T.D., Morey, R., "The Use of Categorical Variables in Data Envelopment Analysis", Management Science, vol.32, no.12, pp. 1613-1627, 1986

[BATT77] Battese, G.E., Corra, G.S., "Estimation of a production frontier model: With application to the pastoral zone of eastern Australia", Australian Journal of Agricultural Economics, vol. 21, no.3, pp. 169-179, 1977

[BAUE93] Bauer, P.W., Fried, H.O., Lovell, C.A.K., Schmidt, S.S., "Efficiency and Productivity Growth in U.S. banking", The Measurement of Productive Efficiency: Techniques and Applications, Oxford University Press, pp. 386-413, 1993

[BAUE98] Bauer, P.W., Berger A.N., Ferrier, G.D., Humphrey, D.B., "Consistency conditions for regulatory analysis of financial institutions: A comparison of frontier efficiency methods", Journal of Economics and Business, vol.50, pp.85114, 1998

[BCG10] Walsh, I., Forth, P., Thogmartin, S., Bickford, J., "Building a High-Powered Branch Network in Retail Banking", The Boston Consulting Group, March $8^{\text {th }}$, 2010.

[BERG07] Berger, A.N., "International Comparisons of Banking Efficiency", Financial Markets, Institutions \& Instruments, vol.16, pp.119-144, 2007

[BERG91] Berger, A.N., Humphrey, D.B., "The dominance of inefficiencies over scale and product mix economies in banking", Journal of Monetary Economics, vol.28, pp.117-148, 1991

Multi-Stage Evaluation of Bank Branch Consolidation and Growth Potential Using DEA 
[BERG93] Berger, A.N., Hunter, W.C., Timme, S.G., "The efficiency of financial institutions: A review and preview of research past, present and future", Journal of Banking and Finance, vol.17, pp.221-249, 1993

[BERG97] Berger, A.N., Humphrey, D.B., "Efficiency of Financial Institutions: International survey and directions for future research", European Journal of Operations Research, vol.98, pp.175-212, 1997

[BERG97a] Berger, A.N., Leusner, J.H., Mingo, J.J., "The efficiency of bank branches", Journal of Monetary Economic, vol.40, pp.141-162, 1997

[BERG97b] Berger, A. N., and Mester, L. J., "Beyond the black box: What explains differences in the efficiencies of financial institutions?" Journal of Banking and Finance, vol.21, no.7, pp.895-947, 1997

[BERG97c] Berger, A.N., DeYonng, R., "Problem loans and cost efficiency in commercial banks", Journal of Banking and Finance, vol.21, pp.849-870, 1997

[BERG98] Berger, A.N., and Hannan, T.H., "The Efficiency Cost of Market Power in the Banking Industry: A Test of the 'Quiet Life' and Related Hypotheses”, Review of Economics and Statistics, vol.80, pp.454-465, 1998

[BOWL85] Bowlin, W.F., Charnes, A., Cooper, W.W., Sherman, H.D., "Data envelopment analysis and regression approaches to efficiency estimation and evaluation", Annals of Operations Research, vol.2, pp.113-138, 1985

[CAMA05] Camanho, A.S., Dyson, R.G., "Cost efficiency, production and value-added models in the analysis of bank branch performance", Journal of the Operational Research Society, vol.56, pp.483-494, 2005

[CBA10] Canadian Bankers Association, 2010 Banking Statistics: Online Document, http://www.cba.ca, Cited: January $31^{\text {st }}, 2012$

[CDIC12] Canada Deposit Insurance Corporation, Online Source: http://www.cdic.ca, Cited: February $23^{\text {rd }}, 2012$

[CHAR78] Charnes, A., Cooper, W., \& Rhodes, E., "Measuring the Efficiency of Decision Making Units", European Journal of Operations Research, vol.2, no.6, pp.429444, 1978

[CHAR82] Charnes, A., Cooper, W.W., Seiford, L., Stutz, J., "A Multiplicative Model for Efficiency Analysis”, Socio-Econ Plan. Sci., vol.16, no.5, pp.223-224, 1982

[CHAR83] Charnes, A., Cooper, W.W., Seiford, L.M., Stutz, J., "Invariant multiplicative efficiency and piecewise Cobb- Douglas envelopments", Operations Research Letters, vol.2, pp.101-103, 1983

[CHAR85] Charnes, A., Cooper, W.W., Golany, B., Seiford, L., "Foundations of Data Envelopment Analysis for Pareto-Koopmans Efficient Empirical Production Functions", Journal of Econometric, vol.30, pp.91-107, 1985

[CHAR89] Charnes, A., Coopers, W.W., Wei, Q.L., Huang, Z.M., "Cone Ratio Data Envelopment Analysis and Multi-Objective Programming", International Journal of systems Science, vol. 20, no.7, pp.1099-1118, 1989

[CHAR94] Charnes, A., Cooper, W., et al., "Data Envelopment Analysis: Theory, Methodology and Application", Kluwer Academic Publishers, 2000

[CLAR96] Clark, J.A., "Cost, Scale Efficiency, and Competitive Viability in Banking", Journal of Money, Credit and Banking, vol.28, no.3, part 1, pp.342-364, 1996

[COEL05] Coelli, T.J., O’Donnell, C.J., Battese, G.E., “An Introduction to Efficiency and Productivity Analysis", Springer, $2^{\text {nd }}$ Ed., 2005

[COEL98] Coelli, T., Prasada Rao, D.S., Battese, G.E., "An Introduction to Efficiency and Productivity Analysis", Kluwer Academic Publishers, Boston, 1998 
[COLW92] Colwell, R.J., Davis, E.P., “Output and Productivity in Banking”, Scandanavian Journal of Economics, vol.94, pp.111-129, 1992

[COOK00] Cook, W.D., Hababou, M., and Tuenter, H.J., "Multicomponent efficiency measurement and shared inputs in data envelopment analysis: an application to sales and service performance in bank branches", Journal of Productivity Analysis, vol. 4, pp.209-224, 2000

[COOK01] Cook, W., Hababou, M., "Sales performance measurement in bank branches", The International Journal of Management Science, vol. 29, pp.299-307, 2001

[COOP07] Cooper, W.W., Seiford, L.M., and Tone, K. (Eds.), "Data Envelopment Analysis: A Comprehensive Text with Models, Applications, References and DEA-Solver Software", Springer Science + Business Media, New York, Second Edition, 2007

[DAVI02] Davies, G., "A history of money: from ancient times to present day", University of Wales Press, 2002

[DEBR51] Debreu, G., "The coefficient of resource utilization”, Econometrica, vol.19, pp.272-292, 1951

[DEKK01] Dekker, D., Post, T., “A quasi-concave DEA model with an application for bank branch performance evaluation", European Journal of Operational Research, vol.132, pp.296-311, 2001

[DEPR84] Deprins, D., Simar, L., Tulkens, H., "Measuring Labor Inefficiency in Post Offices", The Performance of Public Enterprises: Concepts and Measurements, pp.243-267, 1984

[DEYO94] De Young, R., "X-Efficiency and Management Quality in Commercial Banks", Economic and Policy Analysis:Office of the Comptroller of the Currency, E\&PA Working Paper 94-1, 1994

[DEYO98] De Young, R., "Management Quality and X-Efficiency in National Banks", Journal of Financial Services, vol.13, no.1, pp.5-22, 1998

[DJC11] Department of Justice Canada, "Bank Act (S.C. 1991, c.46)", Online Document, http://laws.justice.gc.ca/eng/acts/B-1.01/, Cited: February $23^{\text {rd }}, 2012$

[DYSO88] Dyson, R.G., Thanassoulis, E., "Reducing Weight Flexibility in Data Envelopment Analysis", Journal of Operational Research Society, vol.39, no.6, pp.563-576, 1988

[EISE97] Eisenbeis, R.A., Ferrier, G.D., and Kwan, S. "The Informativeness of Linear Programming and Econometric Efficiency Scores: An Analysis using U.S. banking data", Working paper, Bureau of Business and Economic Research, University of Arkansas, 1997

[EPST89] Epstein, M., \& Henderson, J., "Data Envelopment Analysis for Managerial Control and Diagnosis", Decision Sciences, vol.20, pp.90-119, 1989

[FARE78] Fare, R., Lovell, C.A.K., "Measuring the Technical Efficiency of Production", Journal of Economic Theory, vol.19, pp.150-162, 1978

[FARE85] Fare, R., Grosskopf, S., "A nonparametric cost approach to scale efficiency", The Scandinavian Journal of Economics, vol.87, no.4, pp.594-604, 1985

[FARR57] Farrel, M., "The Measurement of Productive Efficiency", Journal of the Royal Statistic Society, vol.120, pp.253-290, 1957

[FARR62] Farrell, M.J., Fieldhouse, M., "Estimating Efficient Production Functions under Increasing Returns to Scale", Journal of the Royal Statistical Society, vol.125, no.2, pp.252-267, 1962

[FERR90] Ferrier, G.D., and Lovell, C.A.K., "Measuring Cost Efficiency in Banking: Econometric and Linear Programming Evidence", Journal of Econometrics, vol.46, pp.229-245, 1990 
[FETH10] Fethi, M.D., Pasiouras F., "Assessing bank efficiency and performance with operational research and artificial intelligence techniques: A survey", European Journal of Operational Research, vol.204, pp.189-198, 2010

[FRAS09] Fraser, N.M., Jewkes, E.M., Bernhardt, I., Tajima, M., “ Global Engineering Economics: Financial Decision Making for Engineers", Pearson, $4^{\text {th }}$ Ed., 2009

[FRIE96] Fried, H.O., Lovell, C.A.K., Turner, J.A., "An analysis of the performance of university-affiliated credit unions", Computers Operations Research, vol.23, no.4, pp.375-384, 1996

[GIOK08] Giokas, D.I., "Assessing the efficiency in operations of a larger Greek bank Branch network adopting different economic behaviours", Economic Modeling, vol. 25, pp.559-574, 2008

[GREB99] Greboval, D., "Managing fishing Capacity: Selected Papers on Underlying concepts and Issues", FAO Fisheries Technical Paper; Food and Agriculture Organization of the United Nations, vol.386, Appendix XIV, 1999

[GREE90] Greene, W.H., "A gamma-distributed stochastic frontier model", Journal of Econometrics, vol.46, pp.141-163, 1990

[HAO01] Hao, J., Hunter, W.C., Yang, W.K., "Deregulation and efficiency: the case of private Korean banks", Journal of Economics and Business, vol.53, pp.237-254, 2001

[HASA96] Hasan, I., and Hunter W.C., "Efficiency of Japanese multinational banks in the U.S.", Research in Finance, vol.14, pp. 157-173, 1996

[HOLL03] Hollingsworth, B., Smith, P., "Use of Ratios in Data Envelopment Analysis", Applied Economics Letters, vol.10, no.11, pp.733-735, 2003

[IBRD04] International Bank for Reconstruction and Development, "Producer Price Index Manual: Theory and Practice", International Monetary Fund, 2004

[JAHA04] Jahanshahloo, G.R., Amirteimoori, A.R., Kordrostami, S., "Measuring the multicomponent efficiency with shared inputs and outputs in data envelopment analysis", Applied Mathematics and Computation, vol.155, pp.283-293, 2004

[KAPA94] Kaparakis, E.I., Miller, S.M., Noulas, A.G., "Short-run cost inefficiency of commercial banks: A flexible stochastic frontier approach", Journal of Money, Credit and Banking, vol.26, no.4, pp.875-893, 1994

[KINS80] Kinsella, R., "The Measurement of Bank Output", Journal of the Institute of Bankers in Ireland, vol.82, pp.173-83, 1980

[KOOP51] Koopmans, T.C., "Activity Analysis of Production and Allocation”, Wiley, NewYork, 1951

[LEON03] Leong, W.H., Dollery, B., Coelli, T., "Measuring the technical efficiency of banks in Singapore for the period 1993-1999: An application and extension of the Bauer et al. (1997) technique", ASEAN Economic Bulletin, vol.20, no.3, pp.195-210, 2003

[MANA02] Manandhar, R., Tang, J.C.S., "The evaluation of bank branch performance using data envelopment analysis: A framework", Journal of High Technology Management Research, vol.13, pp.1-17, 2002

[MCEA07] McEachern, D., Paradi, J.C., "Intra- and inter-country bank branch assessment using DEA", Journal of Productivity Analysis, vol.27, pp.123-136, 2007

[MEEU77] Meeusen, W., Van Den Broeck, J., "Efficiency estimation from Cobb-Douglas production functions with composed error", International Economic Review, vol.18, no.2, pp.435-444, 1977 
[MIN11] Min, E.J., "Evaluating Customer Service Representative Staff Allocation and Meeting Customer Satisfaction Benchmarks: DEA Bank Branch Analysis”, MASc Thesis, Center for Management of Technology and Entrpreneurship, University of Toronto, 2011

[MURI04] Murillo-Zamorano, L.R, “Economic efficiency and frontier techniques", Journal of Economic Surveys, vol.18, no.1, pp.33-77, 2004

[OSFI11] Office of the Superintendent of Finanncial Institutions Canada, Fact Sheet: Online Document, http://www.osfi-bsif.gc.ca/osfi/, Cited: February $23^{\text {rd }}, 2012$

[PARA04] Paradi, J.C., Schaffnit, C., "Commercial branch performance evaluation and results communication in a Canadian bank- a DEA application”, European Journal of Operational Research, vol.156, pp.719-735, 2004

[PARA10] Paradi, J.C., Vela, S., Zhu, H., “Adjusting for cultural differences, a new DEA model applied to a merged bank", Journal of Productivity Analysis, vol.33, pp.109-123, 2010

[PARA11] Paradi, J.C., Rouatt, S., Zhu, H., "Two-stage evaluation of bank branch efficiency using data envelopment analysis", Omega, vol. 39, pp.99-109, 2011

[PARA12] Paradi, J.C., Zhu, H., "A Survey on Bank Branch Efficiency and Performance Research with DEA”, Omega, 2012

[PARK87] Parkan, C., "Measuring the efficiency of service operations: An application to bank branches, Engineering Costs and Production Economics", vol.12, pp.237242, 1987

[PORT07] Portela, M.C.A.S., Thanassoulis, E., "Comparative efficiency analysis of Portuguese bank branches", European Journal of Operational Research, vol.177, pp.1275-1288, 2007

[REST97] Resti, A., "Evaluating the cost-efficiency of the Italian banking system: What can be learned from the joint application of parametric and nonparametric techniques?", Journal of Banking and Finance, vol.20, pp.221-250, 1997

[SCHA97] Schaffnit, C., Rosen, D., Paradi, J.C., "Best practice analysis of bank branches: An application of DEA in a large Canadian bank", European Journal of Operational Research, vol.98, pp.269-289, 1997

[SEIF96] Seiford, L. "Data Envelopment Analysis: The Evolution of the State of the Art 1978-1995”, Journal of Productivity Analysis, vol.7, pp.99-139, 1996

[SEN90] Sen, A., Srivastava, M., "Regression Analysis: Theory, Methods and Applications", Spinger, 1990

[SHER85] Sherman, H.D., Gold, F., "Bank branch operating efficiency: Evaluation with Data Envelopment Analysis", Journal of Banking and Finance, vol. 9, pp. 297-315, 1985

[SHER95] Sherman, D., Ladino, G., "Managing bank productivity using Data Envelopment Analysis (DEA)", Interfaces, vol.25, pp.60-73, 1995

[SUEY99] Sueyoshi, T., "DEA Duality on Returns to Scale in Production and Cost Analyses: An Occurance of Multiple Solutions and Differences Between Production-Based and Cost-Based RTS Estimates", Management Science, vol.45, no.11, pp.15931608, 1999

[THAN93] Thanassoulis, E., "A omparison of regression analysis and data envelopment analysis as alternative methods for performance assessments", The Journal of the Operations Research Society, vol.44, no.11, pp.1129-1144

[THAN99] Thanassoulis, E., "Data Envelopment Analysis and its use in Banking", Interfaces, vol.29, no.3, pp.1-13, 1999 
[THOM90] Thompson, R.G. et al., "The Role of Multiplier bounds in Efficiency Analysis with Application to Kansas Farming", Journal of Econometrics, vol.46, pp.93108,1990

[TSOL10] Tsolas, I, "Modeling Bank Branch Profitability and Effectiveness by means of DEA", International Journal of Productivity and Performance Management, vol.59, no.5, pp.432-451, 2010

[TULK93] Tulkens, H., "On FDH efficiency analysis: some methodological issues and applications to retail banking, courts and urban transit", Journal of Productivity Analysis, vol.4, pp.183-210, 1993

[WEIL04] Weill, L., "Measuring cost efficiency in European Banking: A comparison of Frontier Techniques", Journal of Productivity Analysis, vol. 21, pp.133-152, 2004

[WU06a] Wu, D., Yang, Z., Liang, L., "Efficiency analysis of cross-region bank branches using fuzzy data envelopment analysis", Applied Mathematics and Computation, vol.181, pp.271-281, 2006

[WU06b] Wu, D., Yang, Z., \& Liang, L., "Using DEA-Neural Network Approach to Evaluate Branch Efficiency of a Large Canadian Bank", Expert Systems with Applications, vol.31, pp.108-115, 2006

[YANG02] Yang, Z., "Handicapping in DEA to Allow for Cultural Differences in DMUs", PhD Thesis, Center for Management of Technology and Entrpreneurship, University of Toronto, 2002

[YUEN93] Yuengert, A., "The measurement of efficiency in life insurance: Estimates of a mixed Normal-Gamma error model", Journal of Banking and Finance, vol.17, pp.483-496, 1993 


\section{Appendices: Appendix 1: Efficiency Distribution Graphs}

\section{0-2011 Period Growth Model:}

CCR Model:
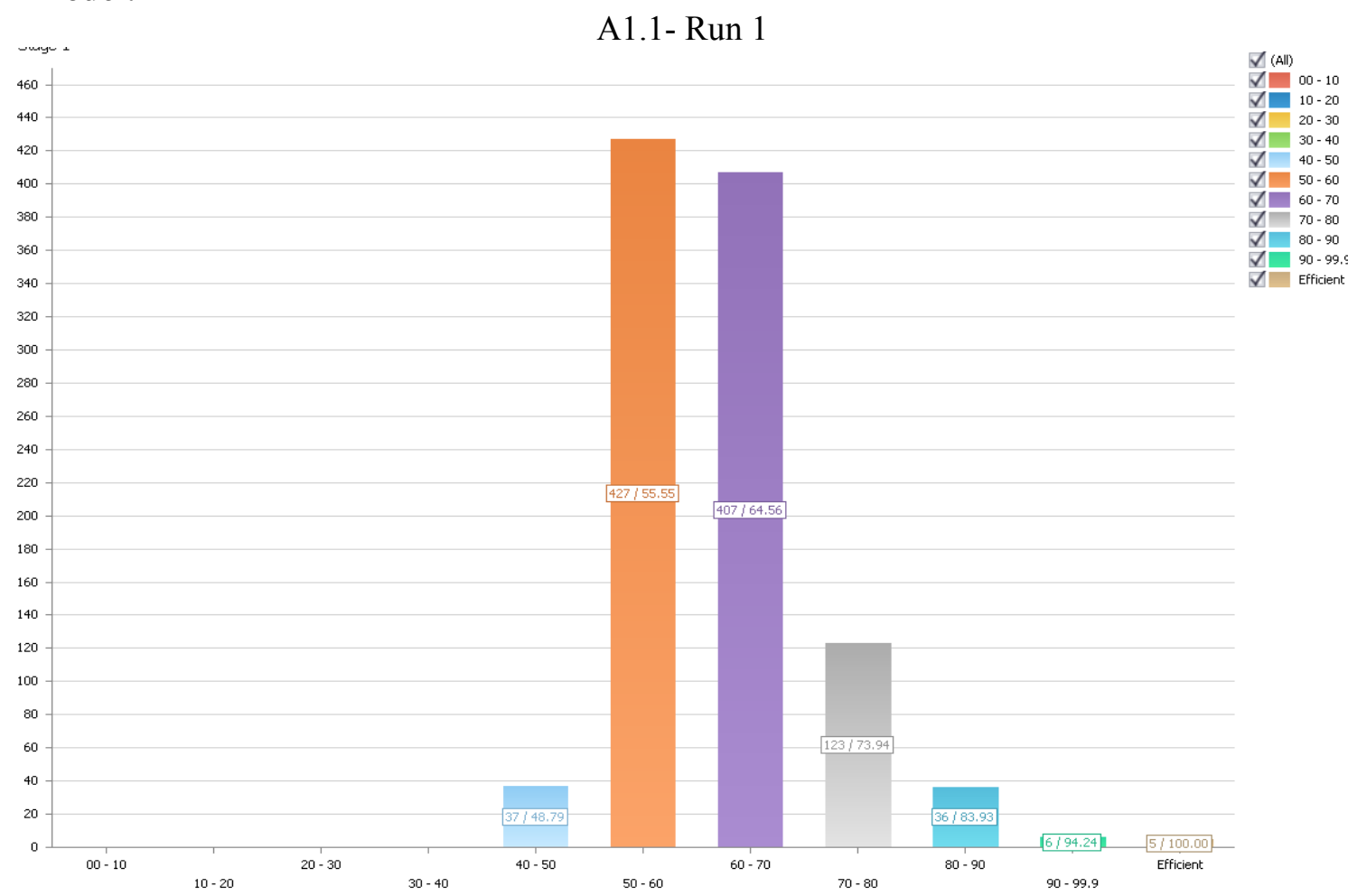

A1.2- Run2

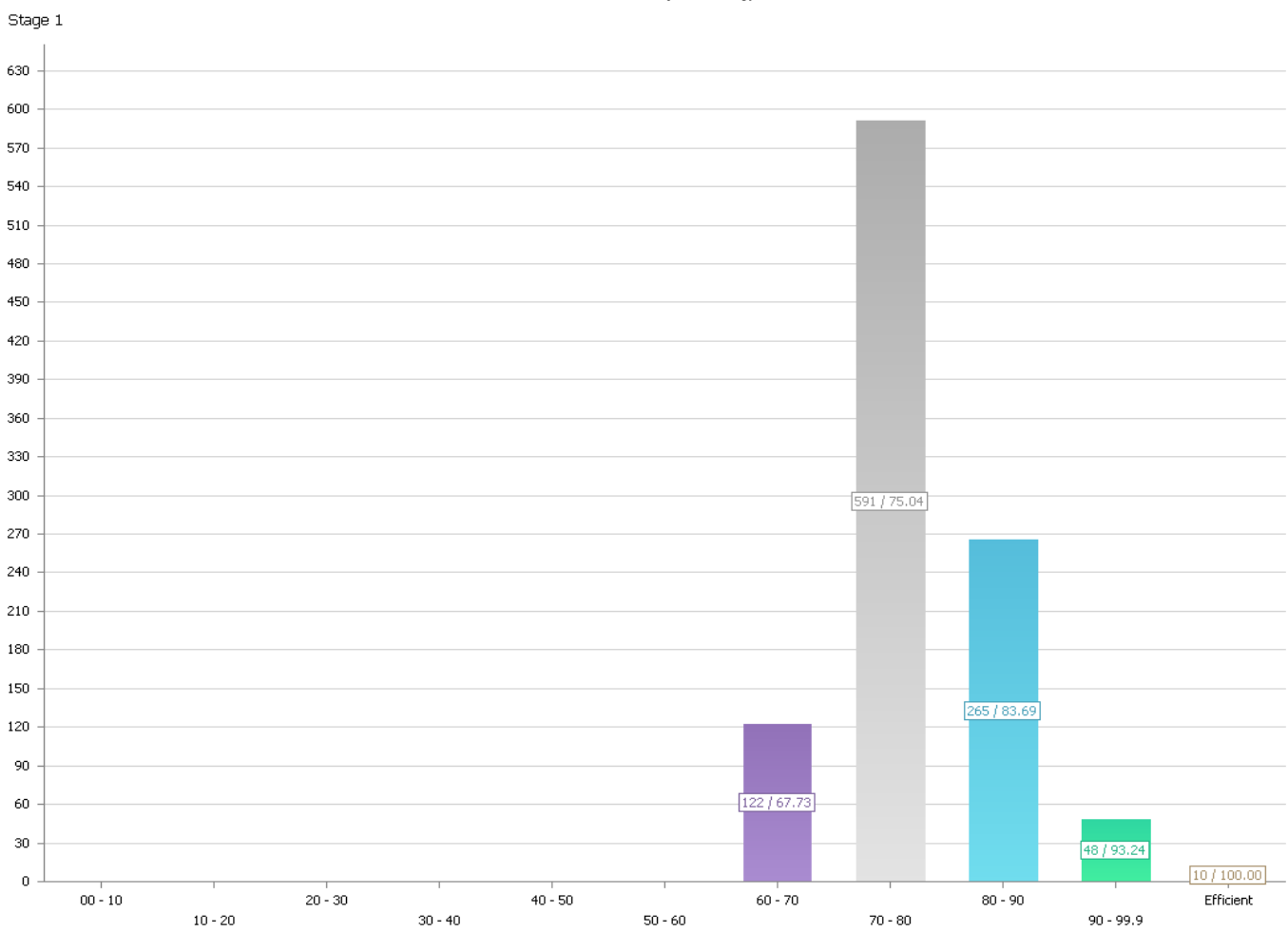

Multi-Stage Evaluation of Bank Branch Consolidation and Growth Potential Using DEA 110 
A1.3- Run3

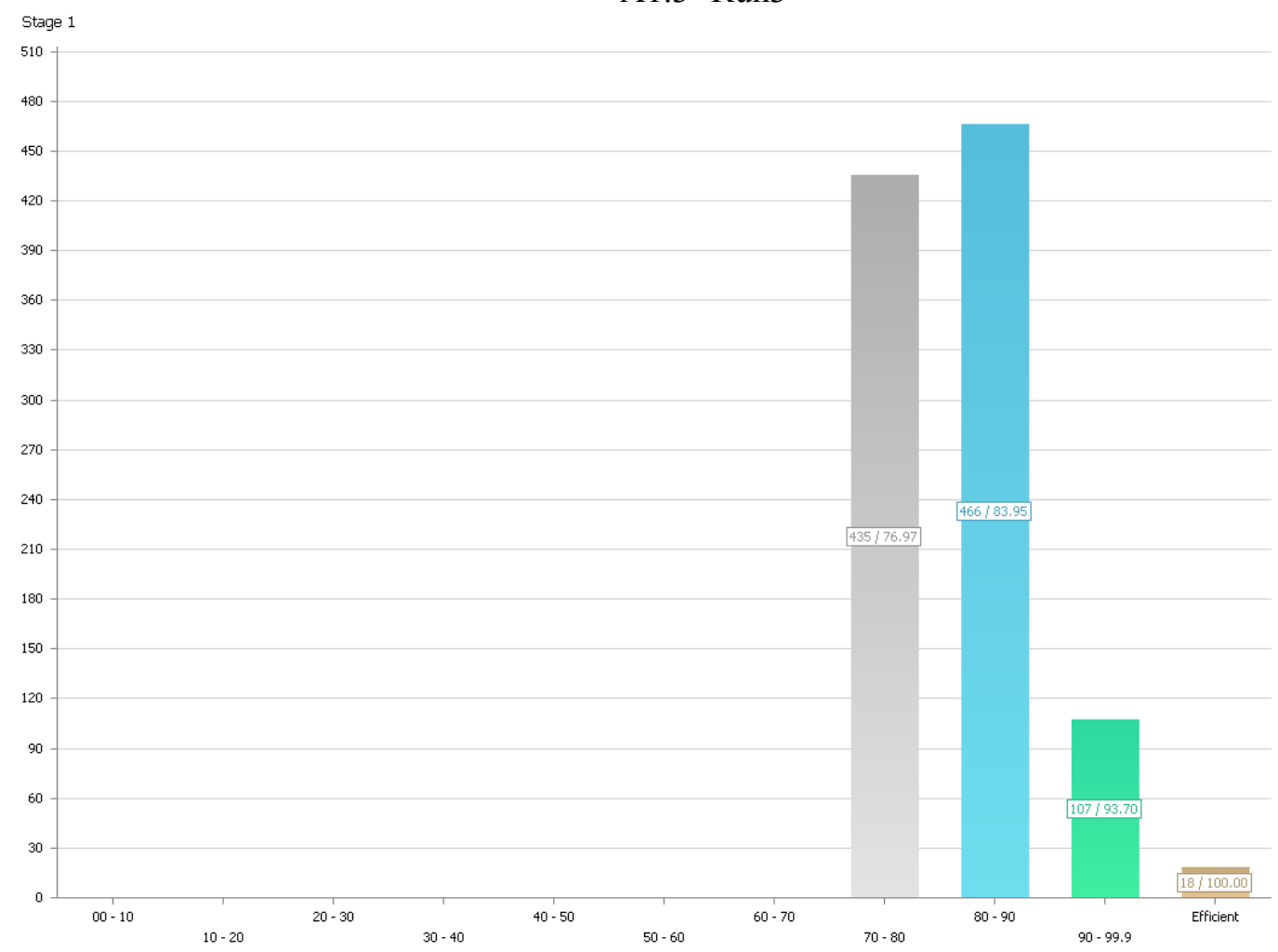

$\checkmark$ (All)

\begin{tabular}{l|l}
$\checkmark$ & $00-10$ \\
7 & $10-20$
\end{tabular}

$\checkmark 20-30$

$\checkmark \quad 30-40$

$\checkmark \quad 40-50$

$50-60$

$60-70$
$7 \quad 70-80$

10

$90-99.9$

$\checkmark$ Efficient

\section{A1.4- Run4}

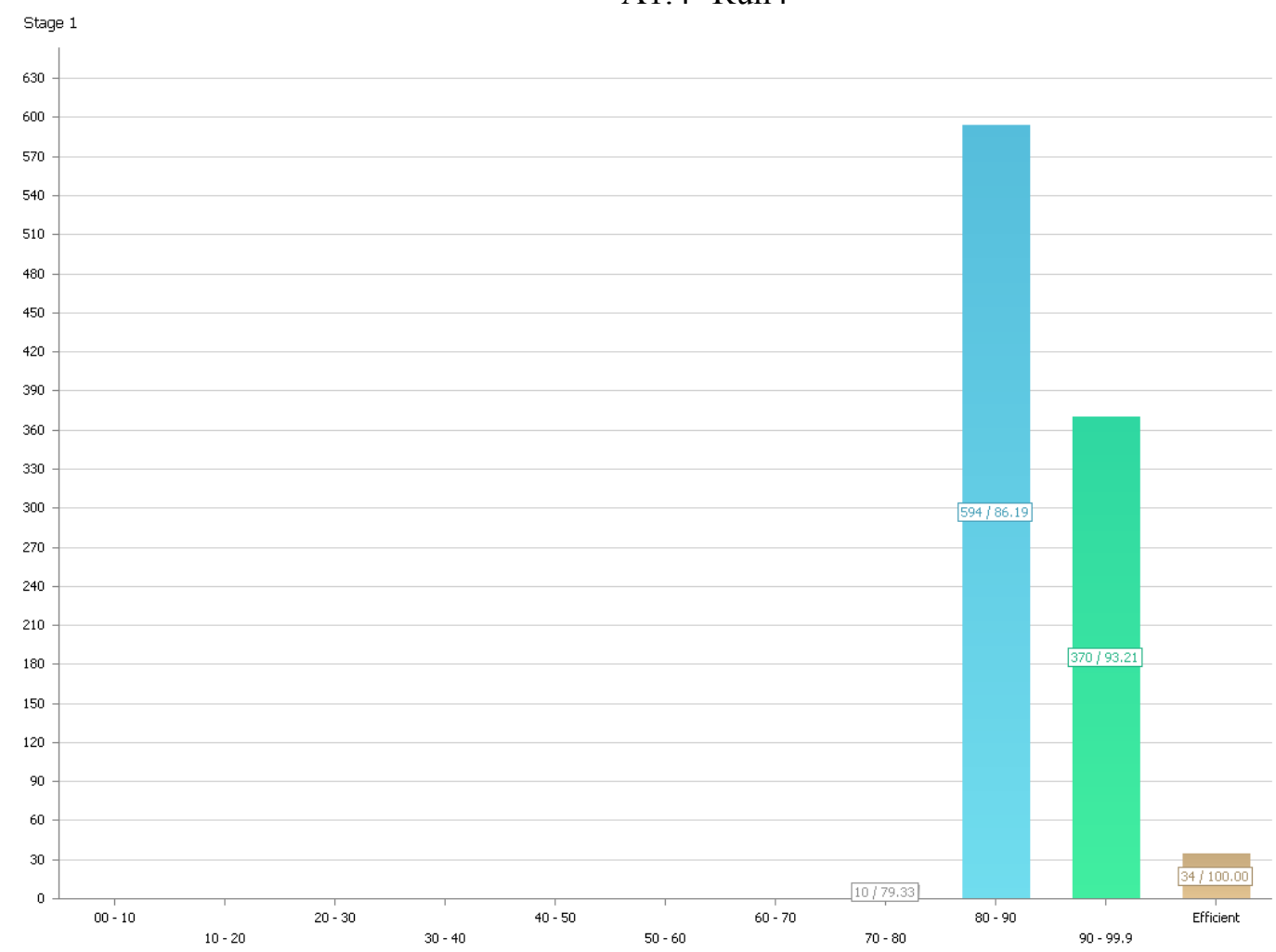




\section{BCC Model:}

A1.5- Run 1

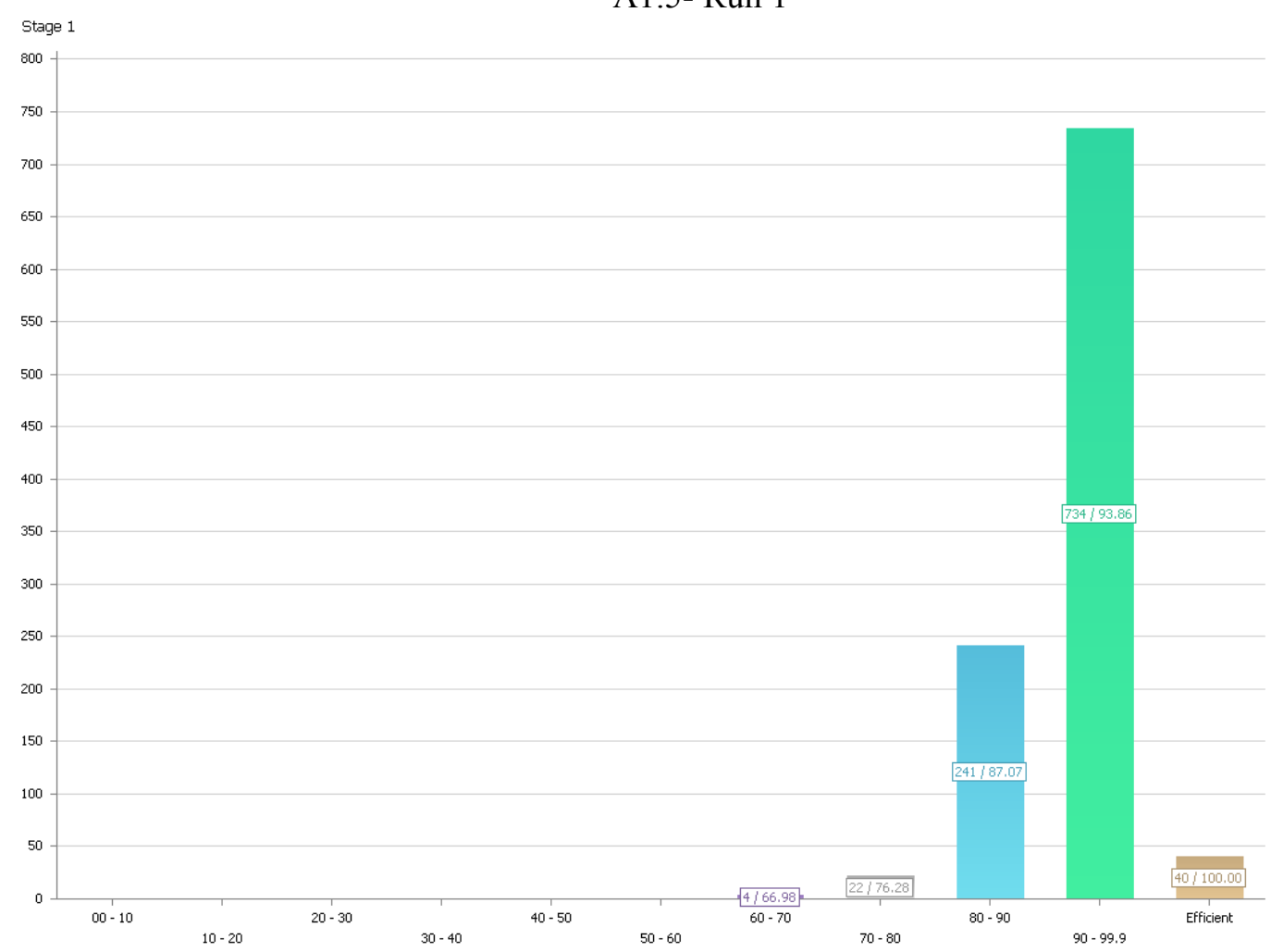

A1.6- Run 2

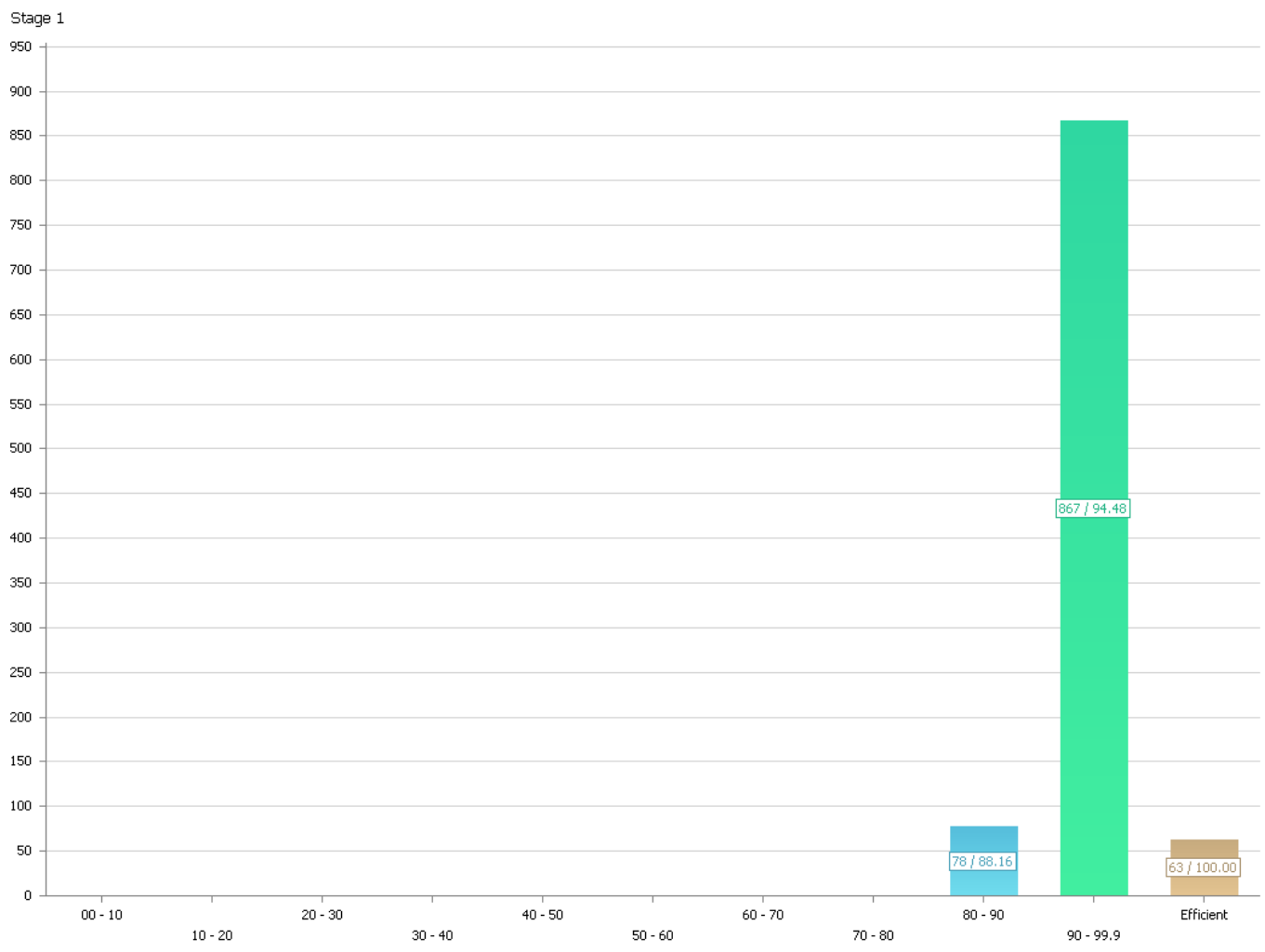




\section{Lost Accounts/Gained Accounts Model}

\section{CCR Model:}

A1.7- Run 1

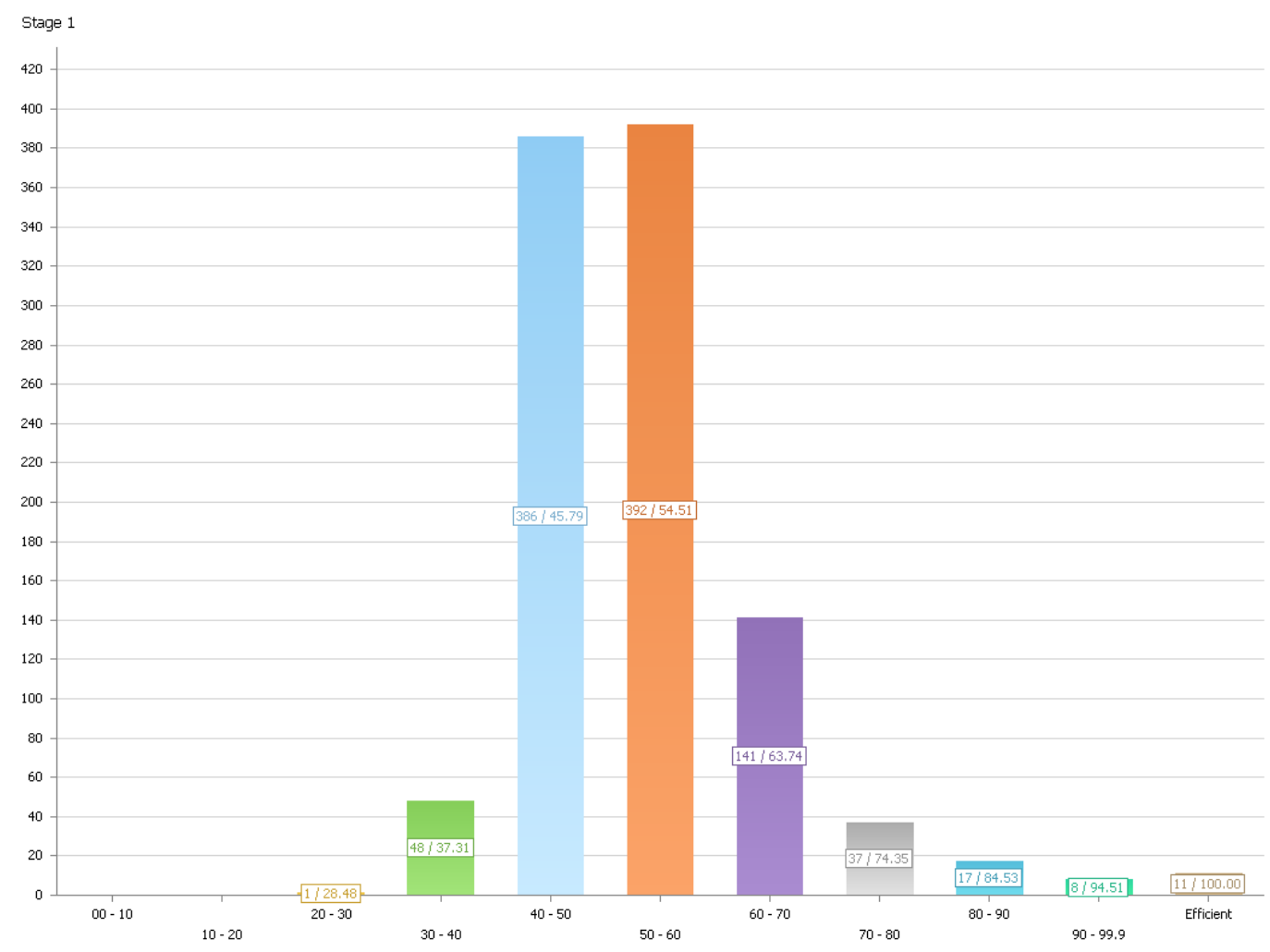

A1.8- Run2

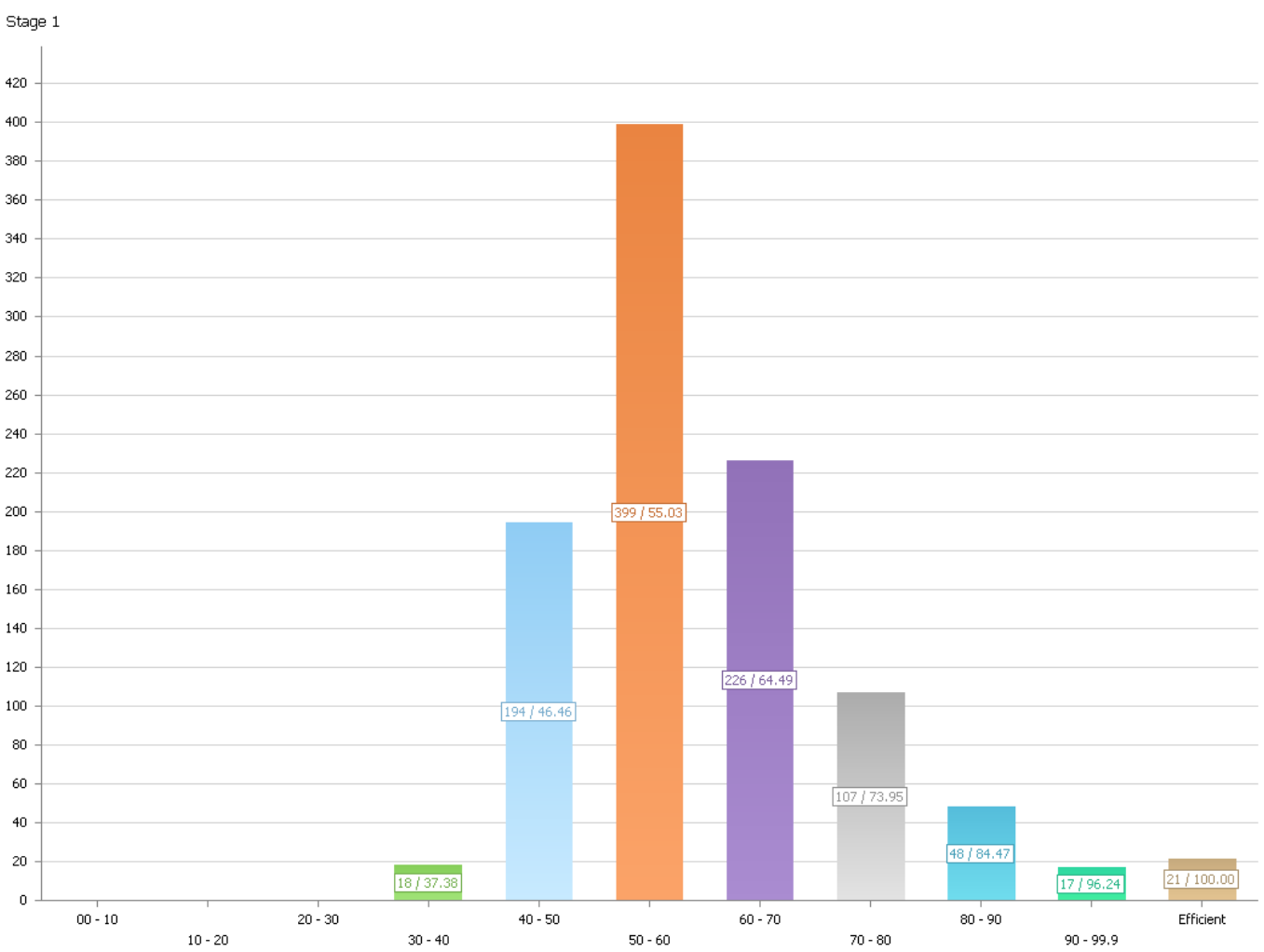




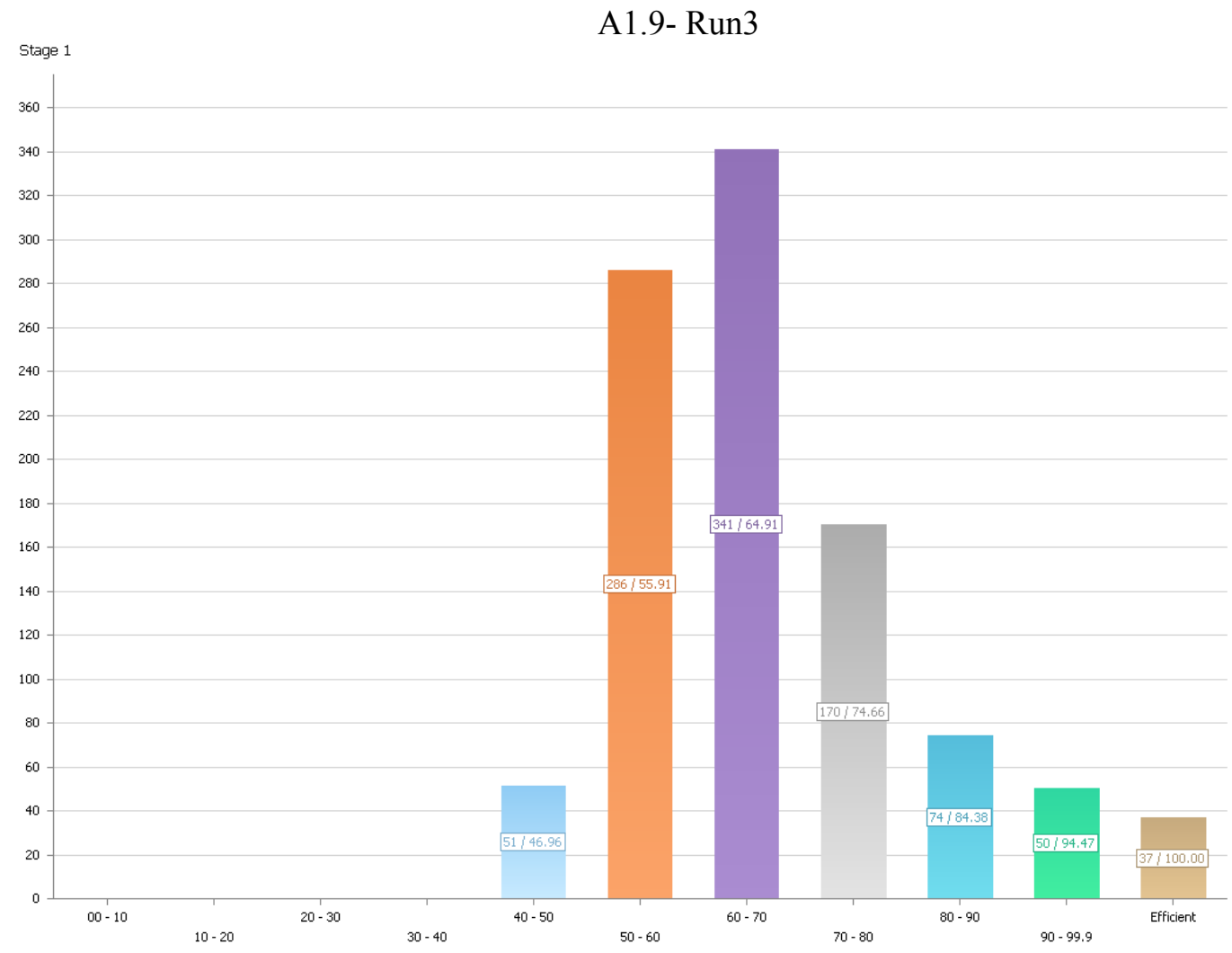

A1.10- Run4

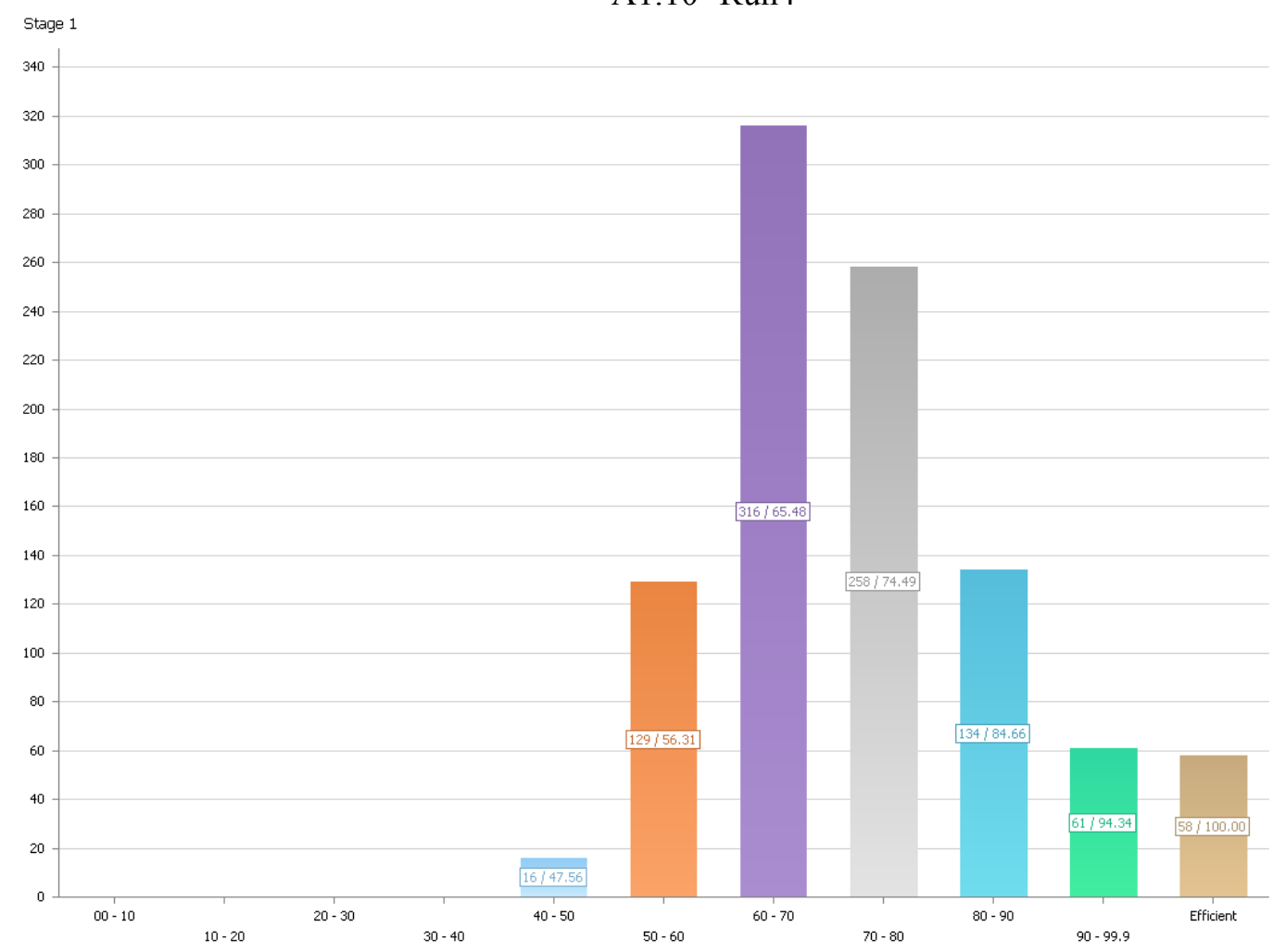




\section{BCC Model:}

A1.11- Run 1

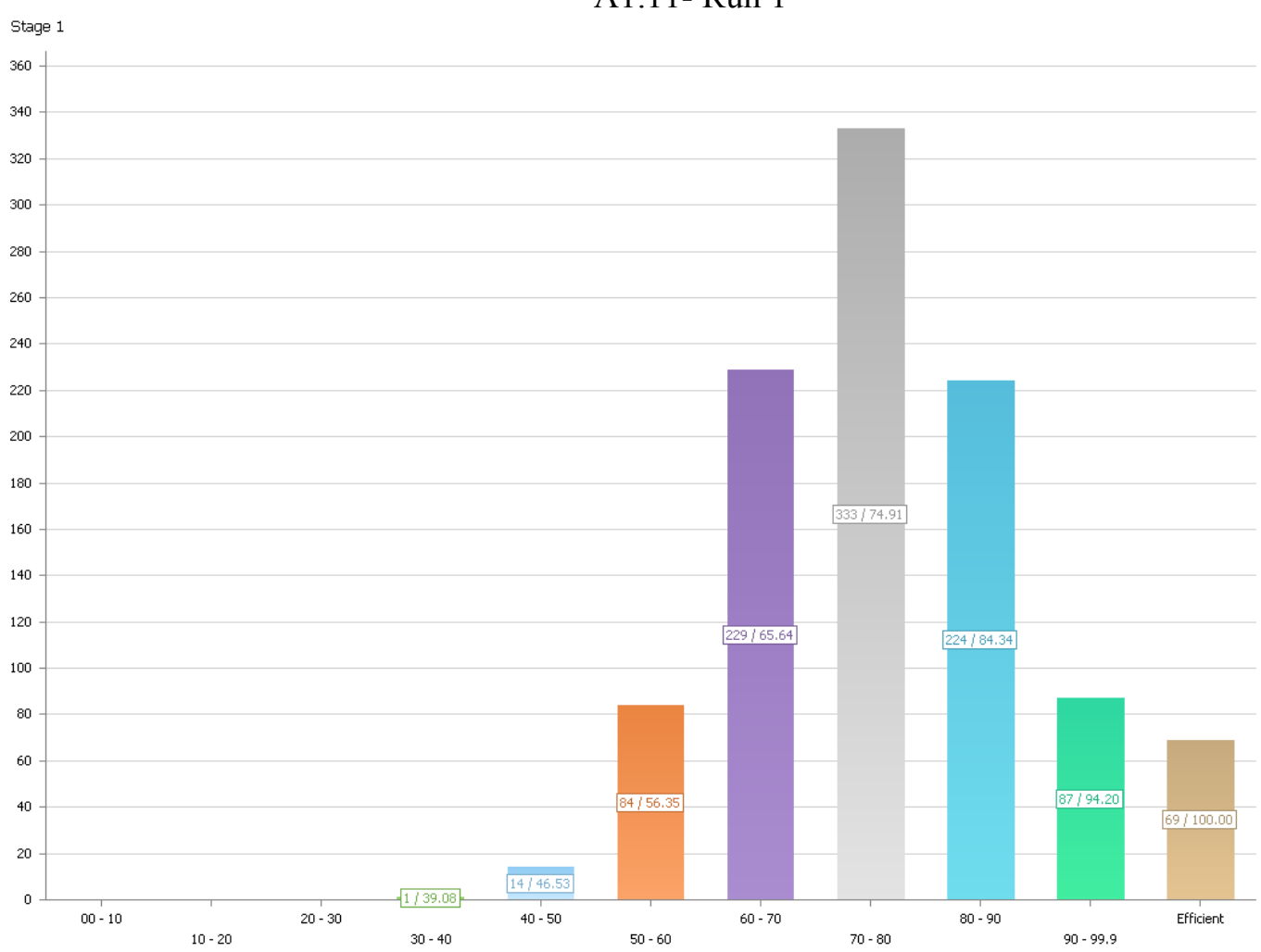

A1.12- Run 2

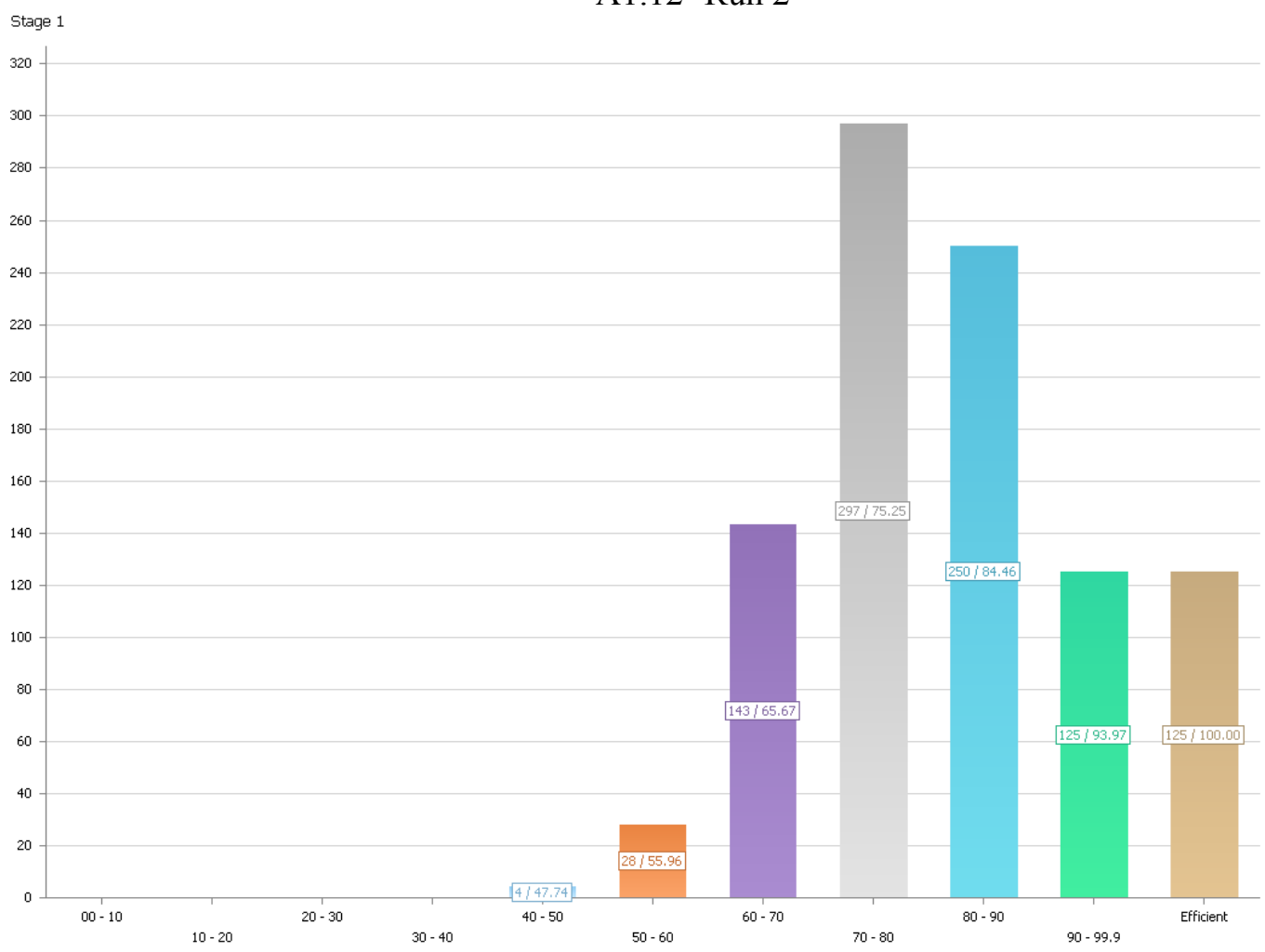




\section{Market Model: Component Model}

\section{CCR Model:}

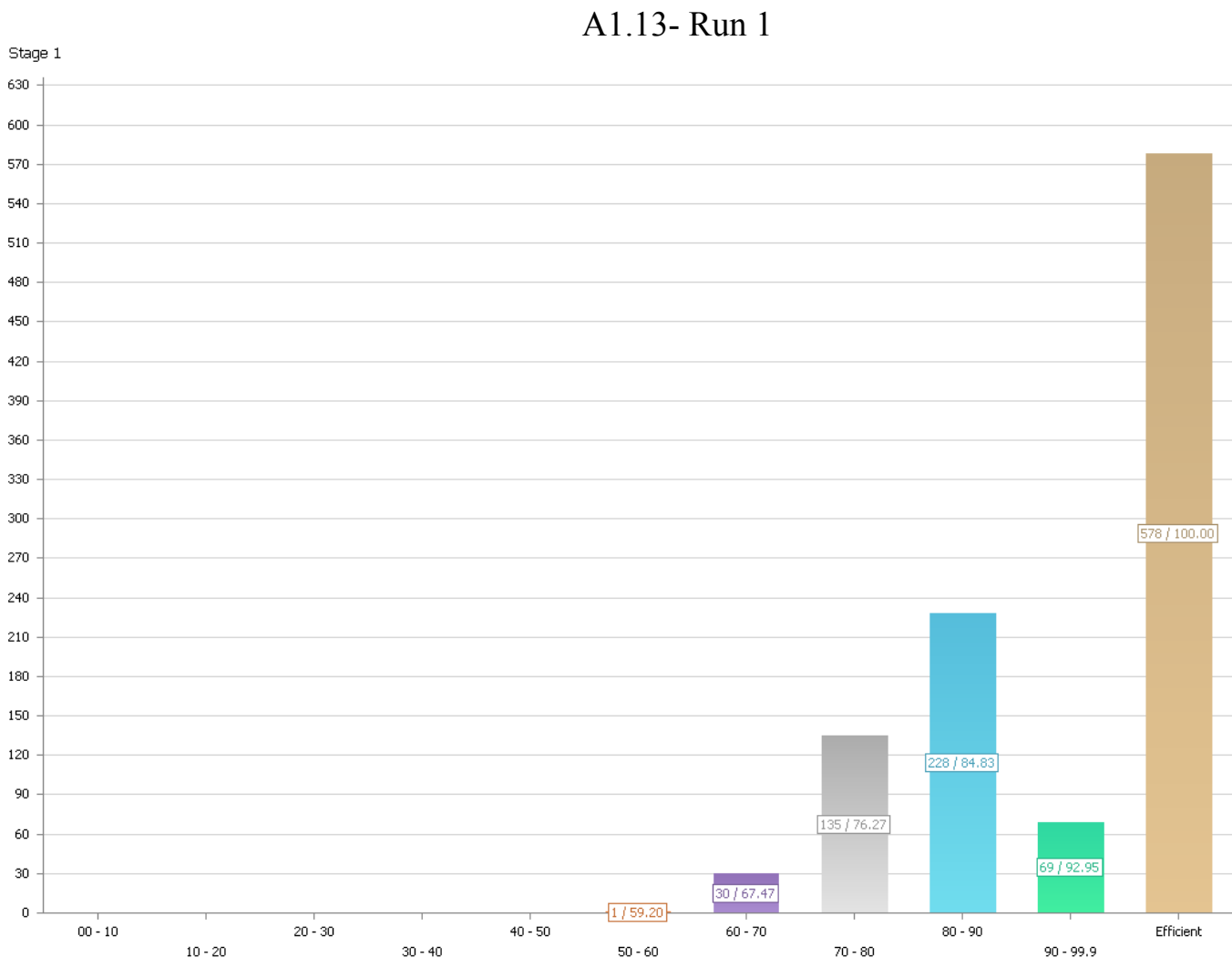

A1.14- Run2

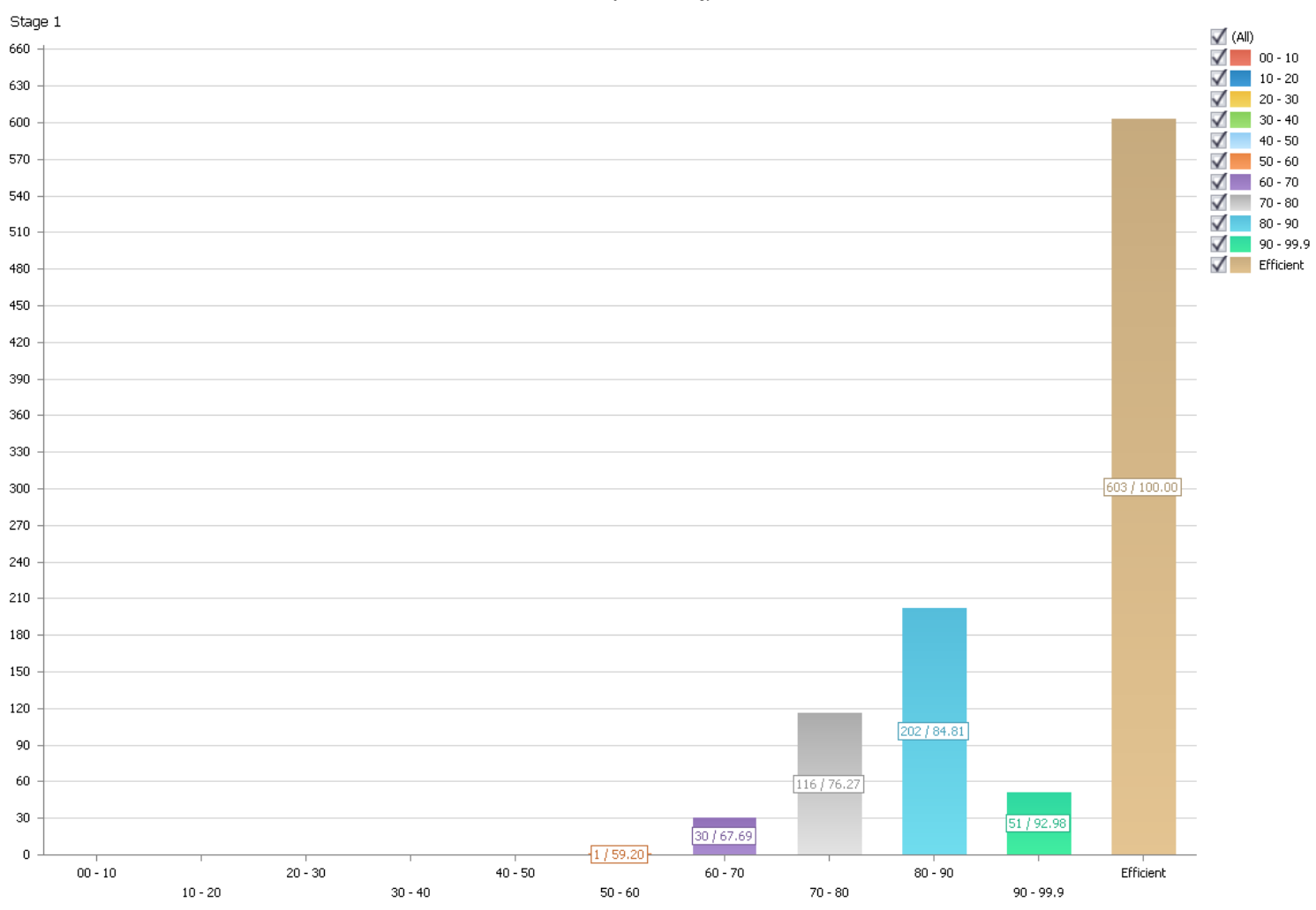




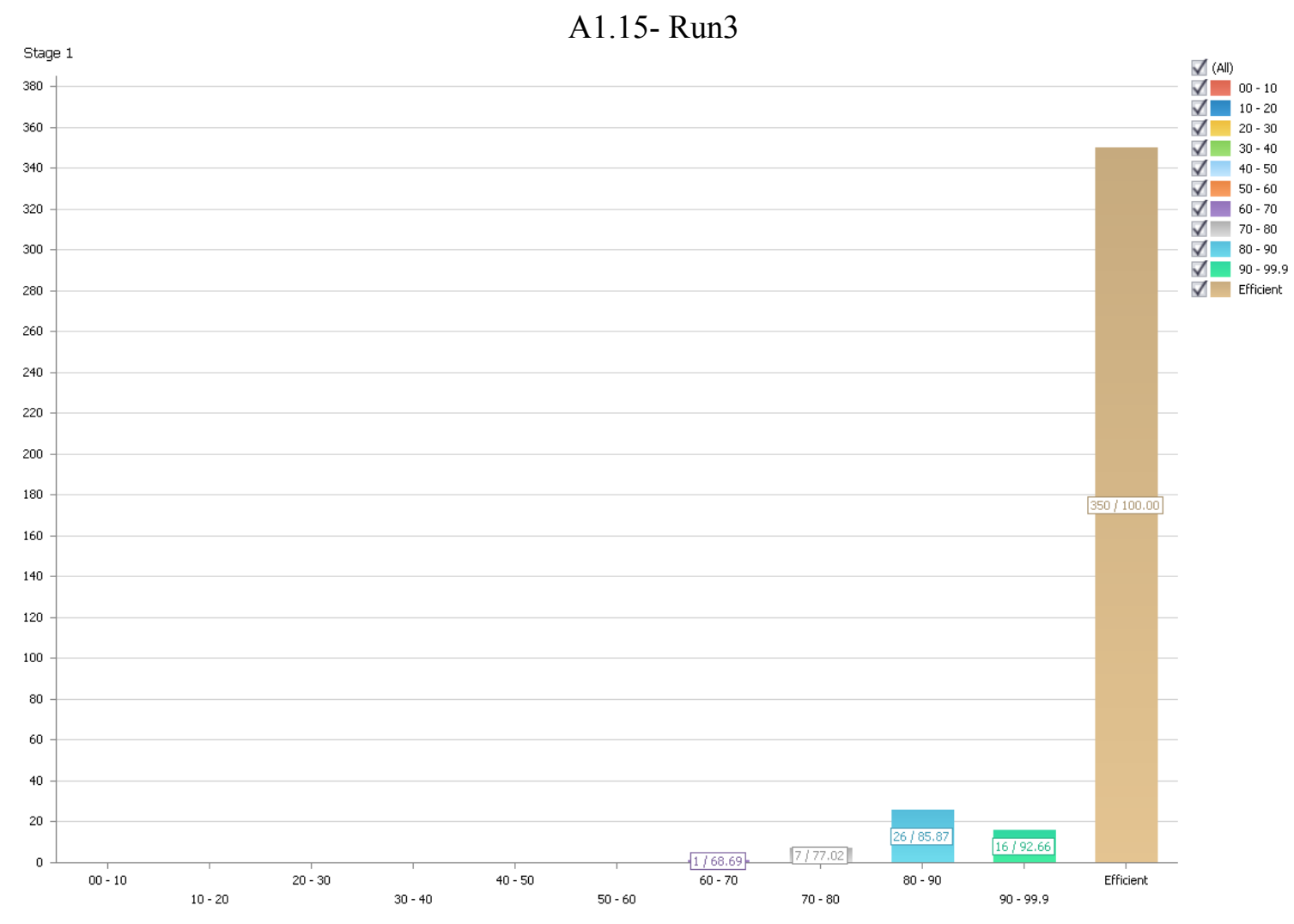

\section{BCC Model}

A1.16- Run 1

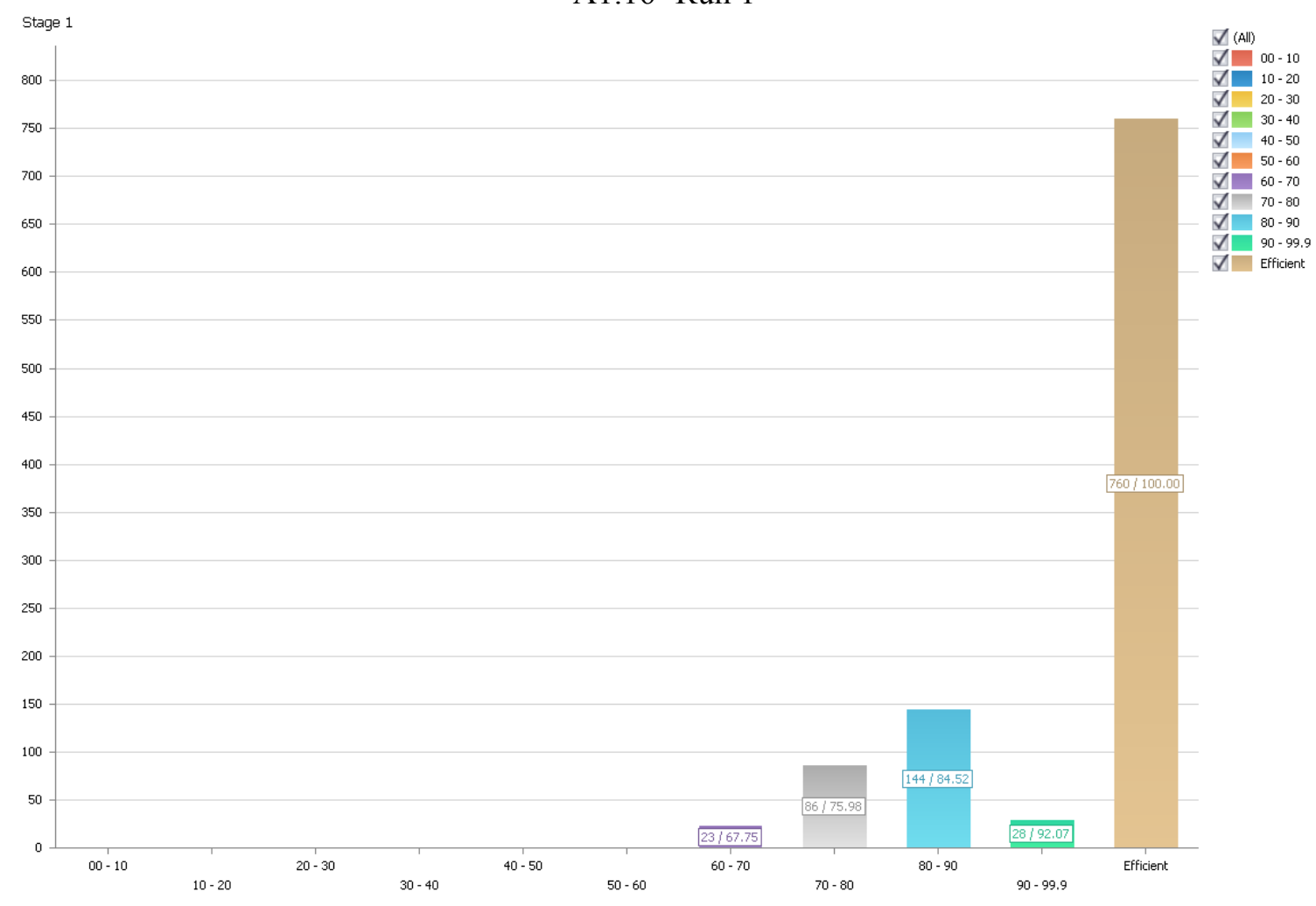


A1.17- Run 2

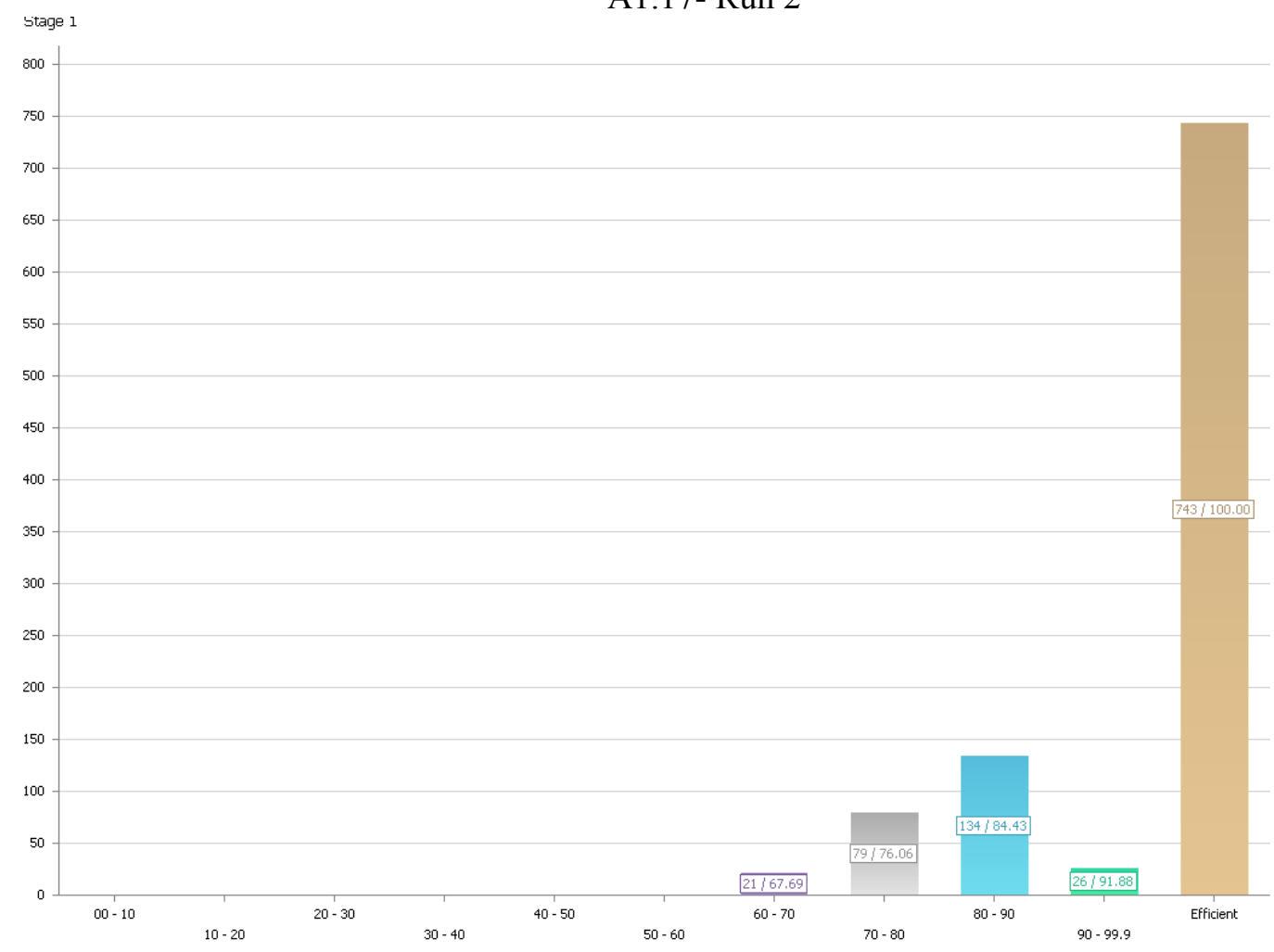

$\checkmark$ (All)

ل $00-10$

$\begin{array}{ll}\checkmark & 10-20 \\ \nabla & 20-30\end{array}$

$\checkmark=30-40$

$\checkmark \quad 40-50$

$\checkmark \quad 50-60$

$\checkmark 60-70$

V $70-80$

$\checkmark \quad 80-90$

7 - $90-99.9$

7 Efficient

A1.18- Run 3

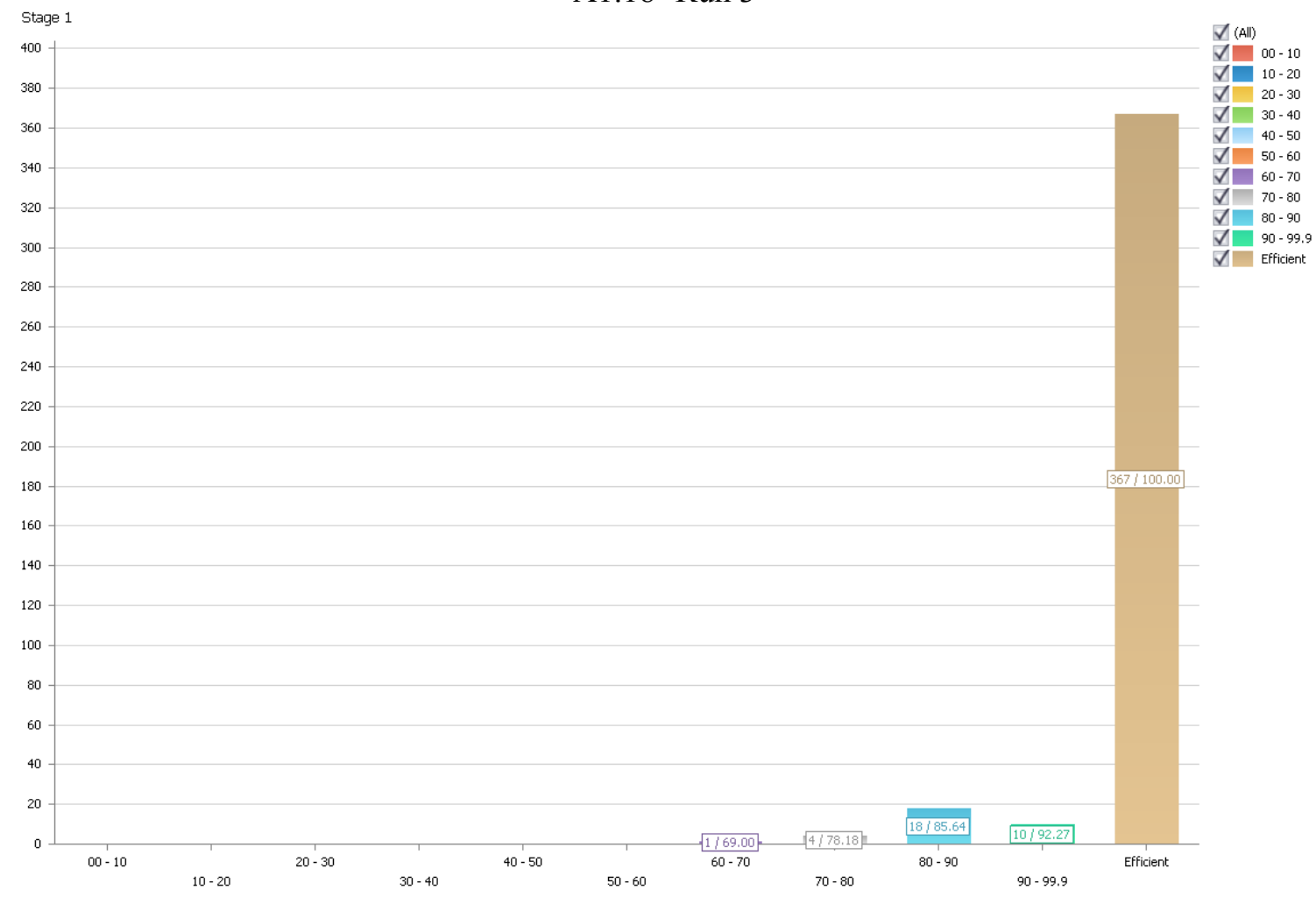




\section{Market Model: Aggregate Model}

\section{CCR Model}

A1.19- Run 1
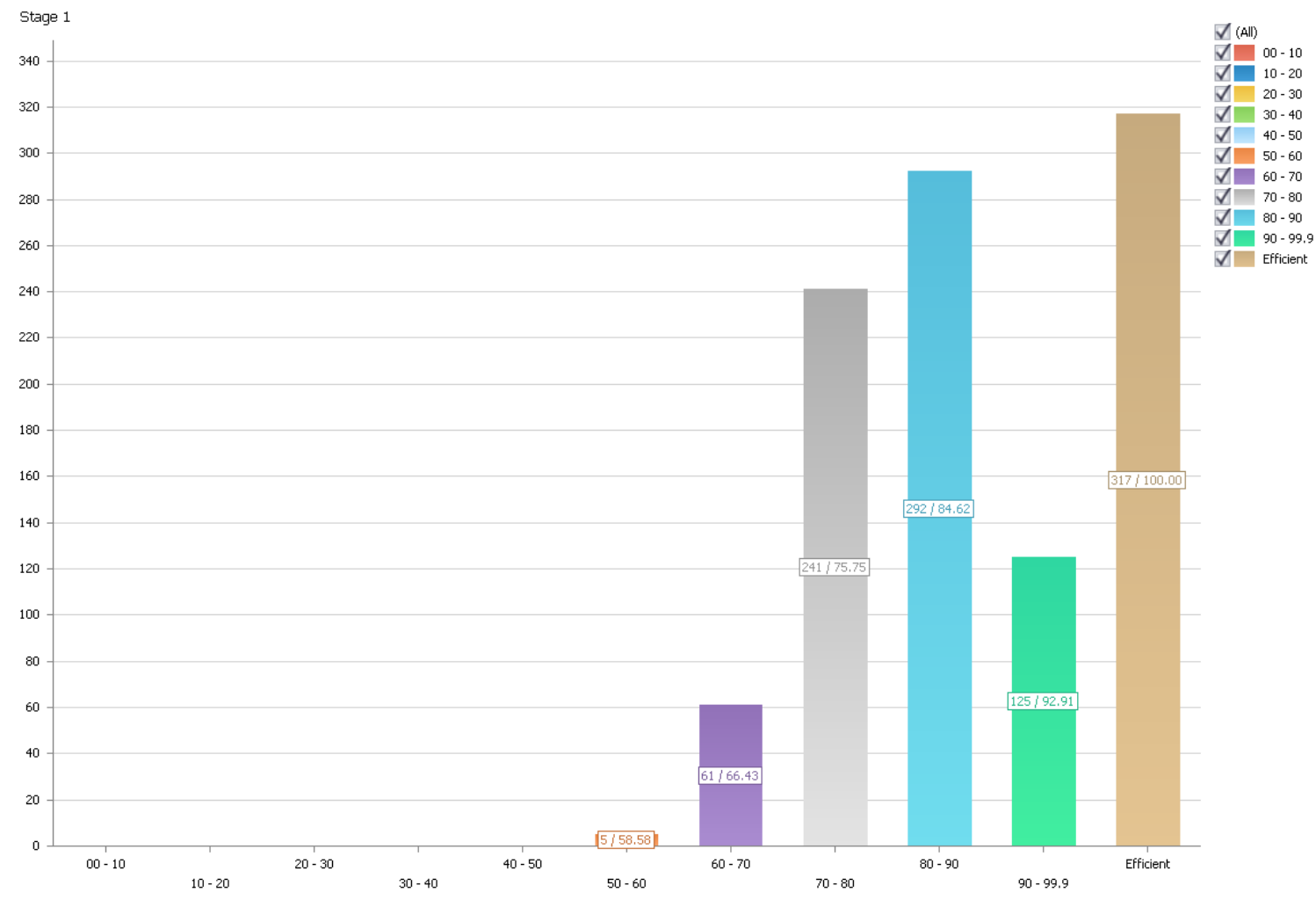

A1.20- Run2

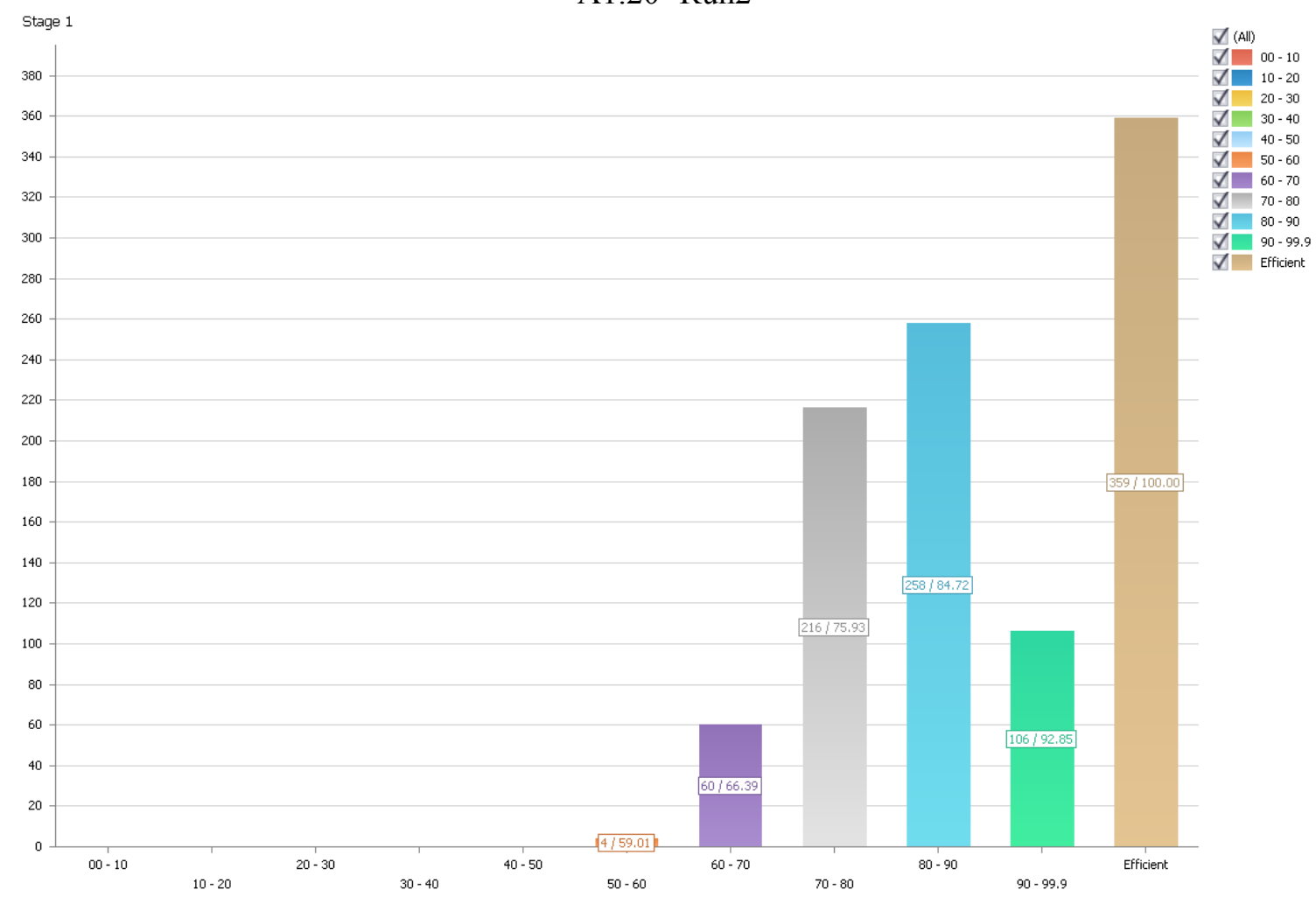


A1.21- Run3

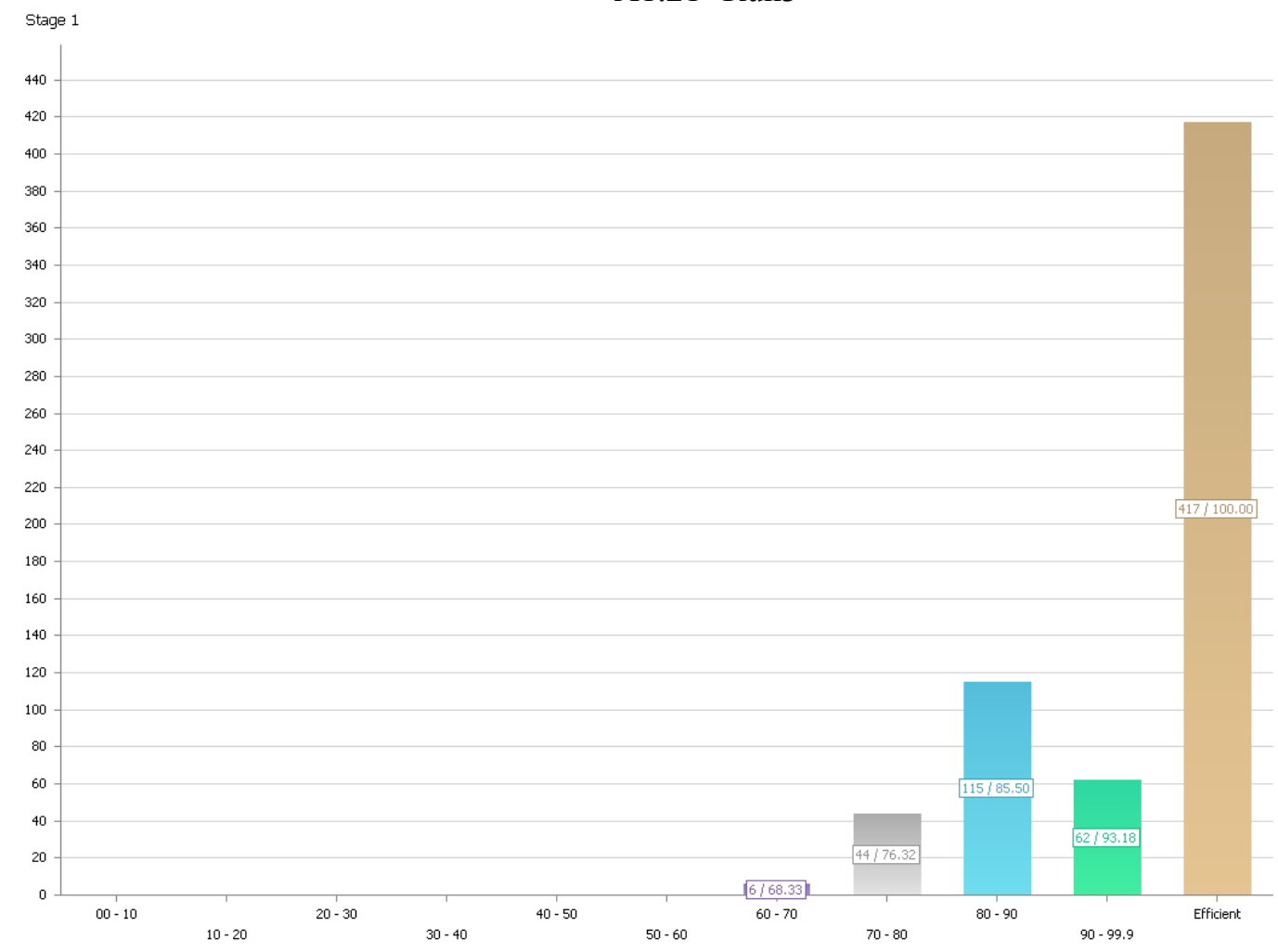

$\checkmark$ (All)

V $\quad 10-20$

$\checkmark \quad 20-30$

$\checkmark \quad 30-40$

V $\quad 40-50$

$\checkmark 60-70$

ᄀ $70-80$

$\checkmark=10-90$

$\begin{array}{ll}\sqrt{ } & 80-90 \\ \nabla & 90-99,9\end{array}$

$\checkmark$ Efficient

\section{BCC Model}

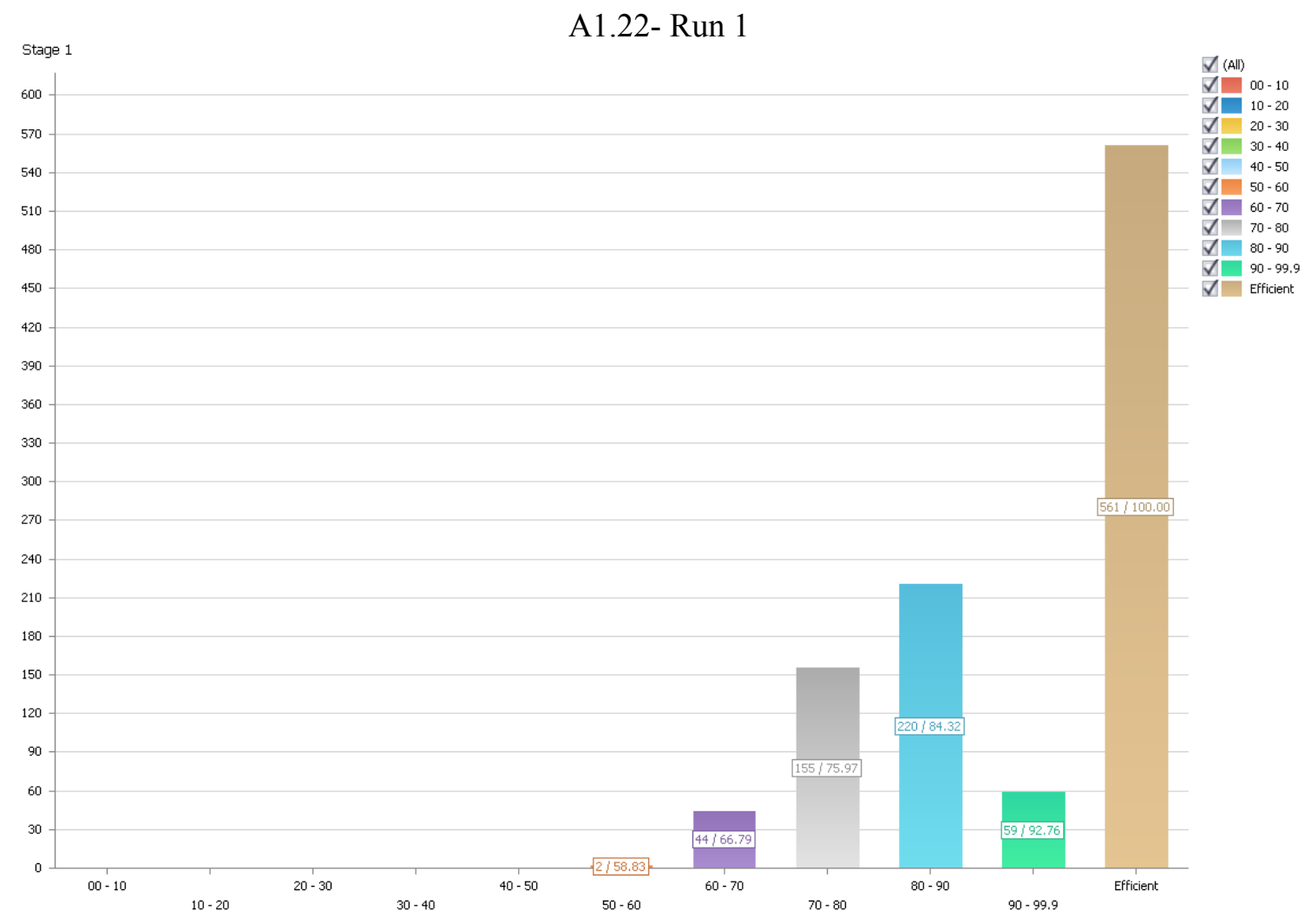


A1.23- Run 2

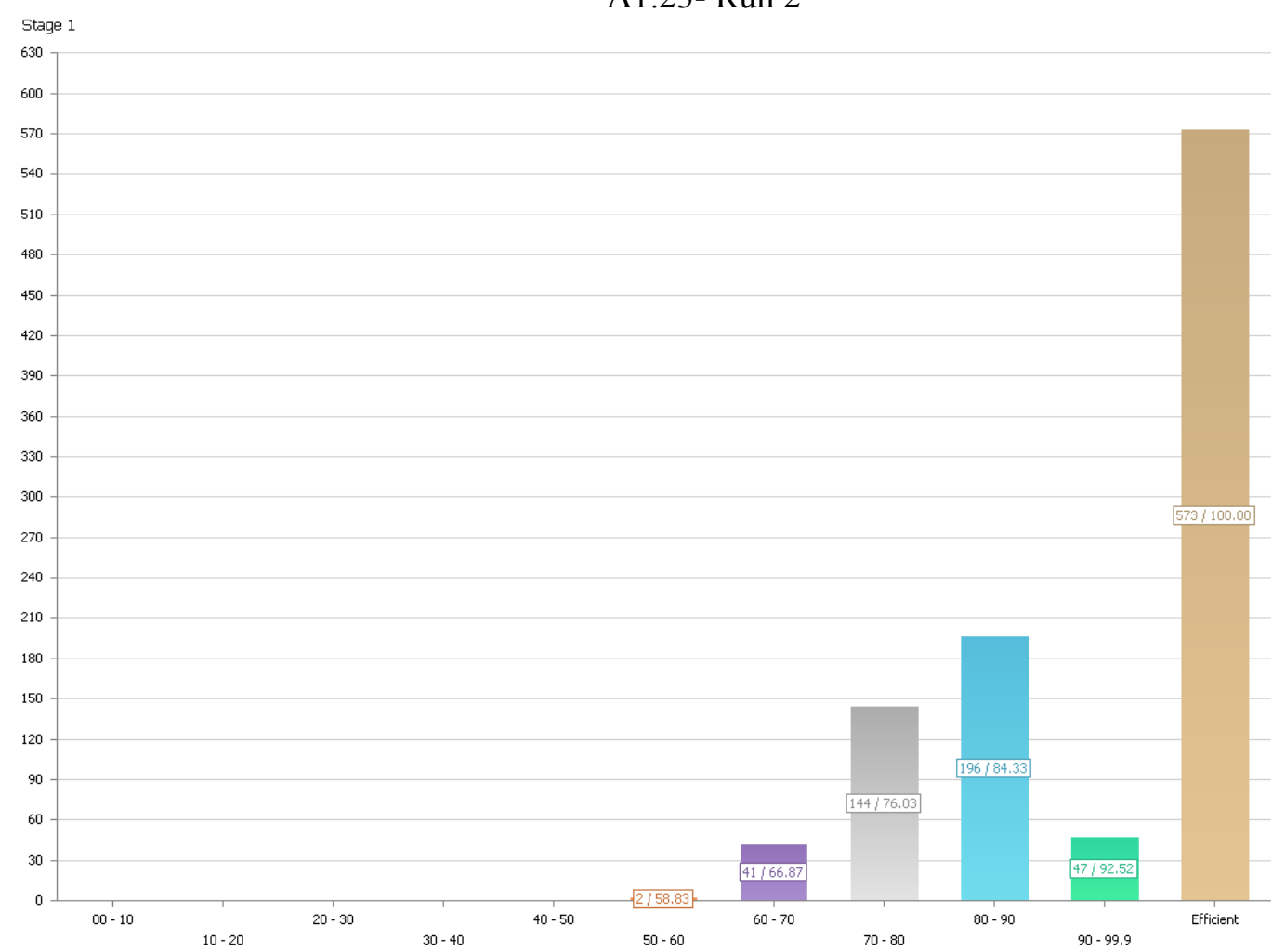

A1.24- Run 3

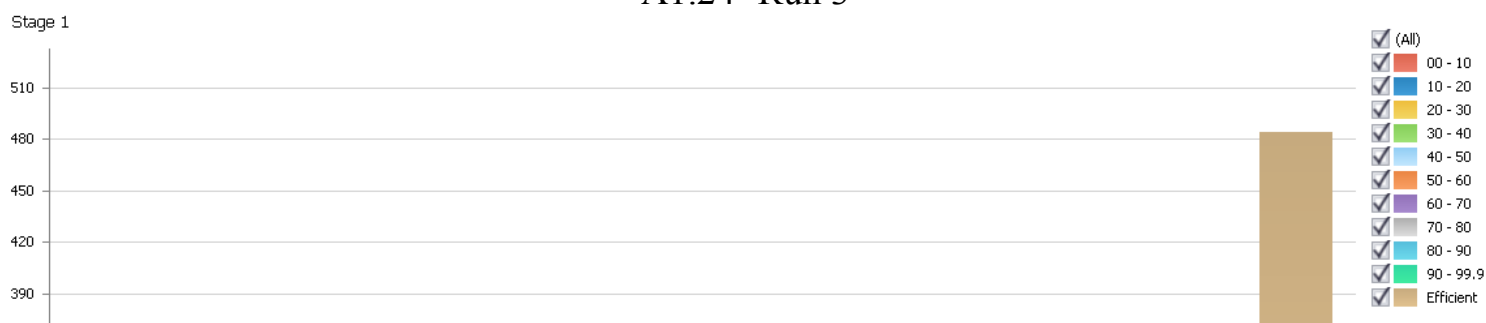




\section{Delta Models: Stripping Efficient Frontier}

\section{Difference Model}

CCR Model

A1.25- Run 1
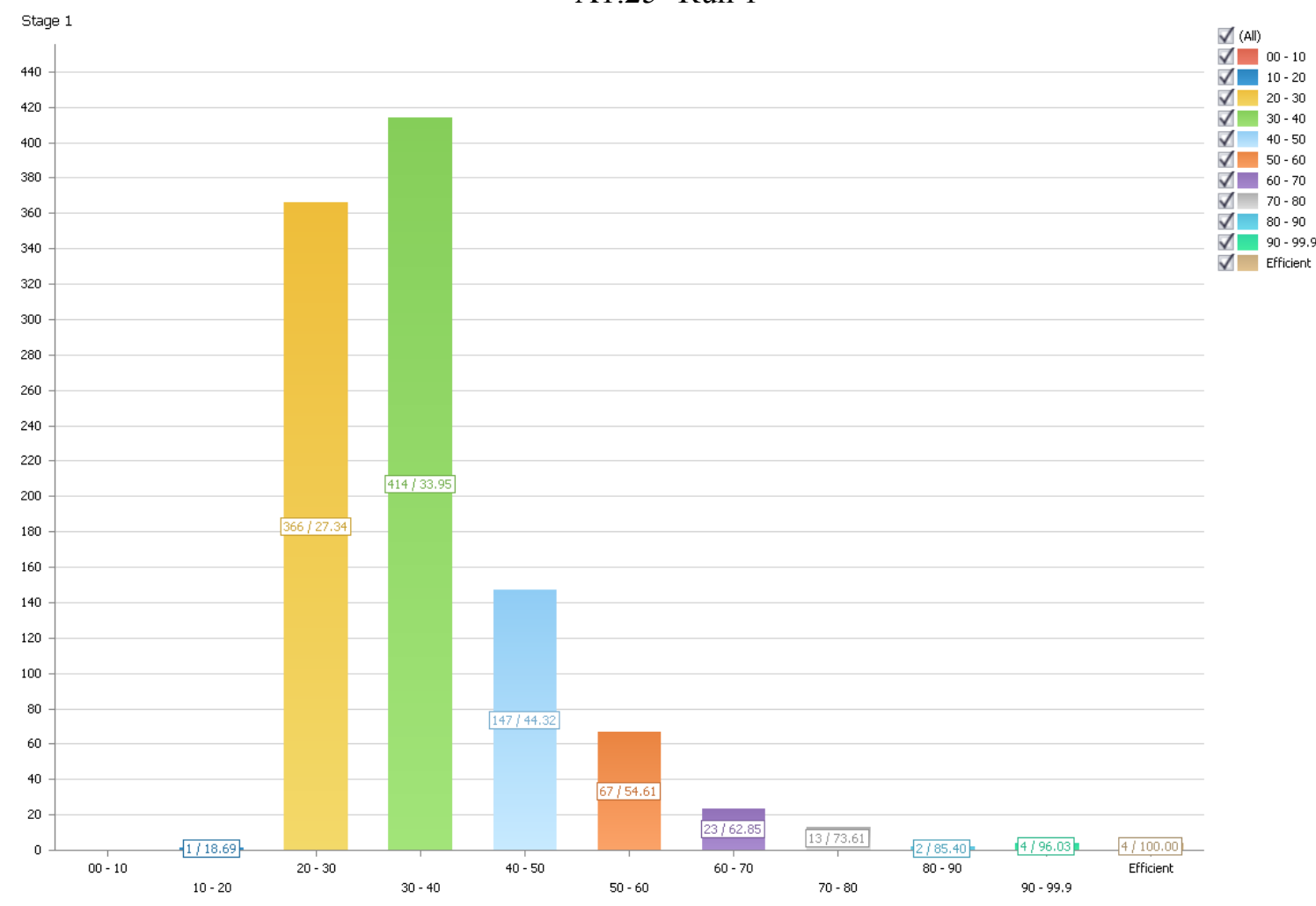

A1.26- Run2

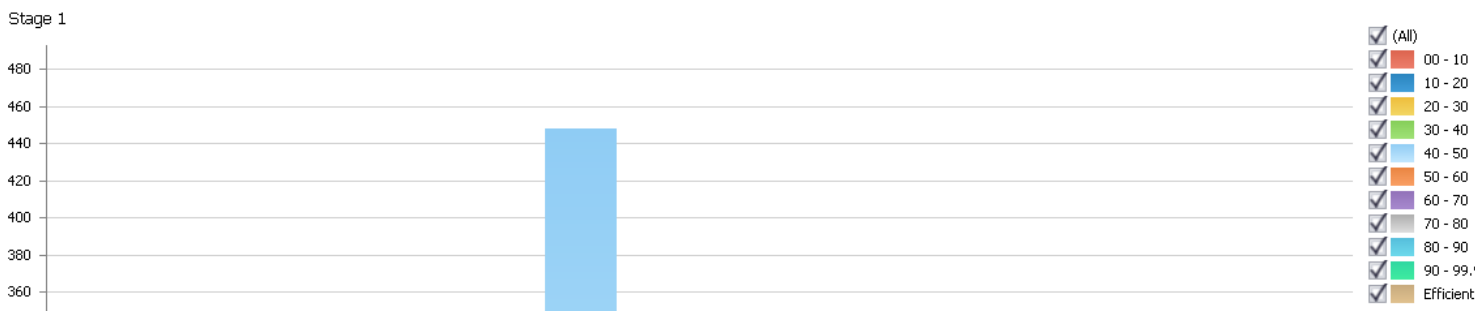




\section{A1.27- Run3}

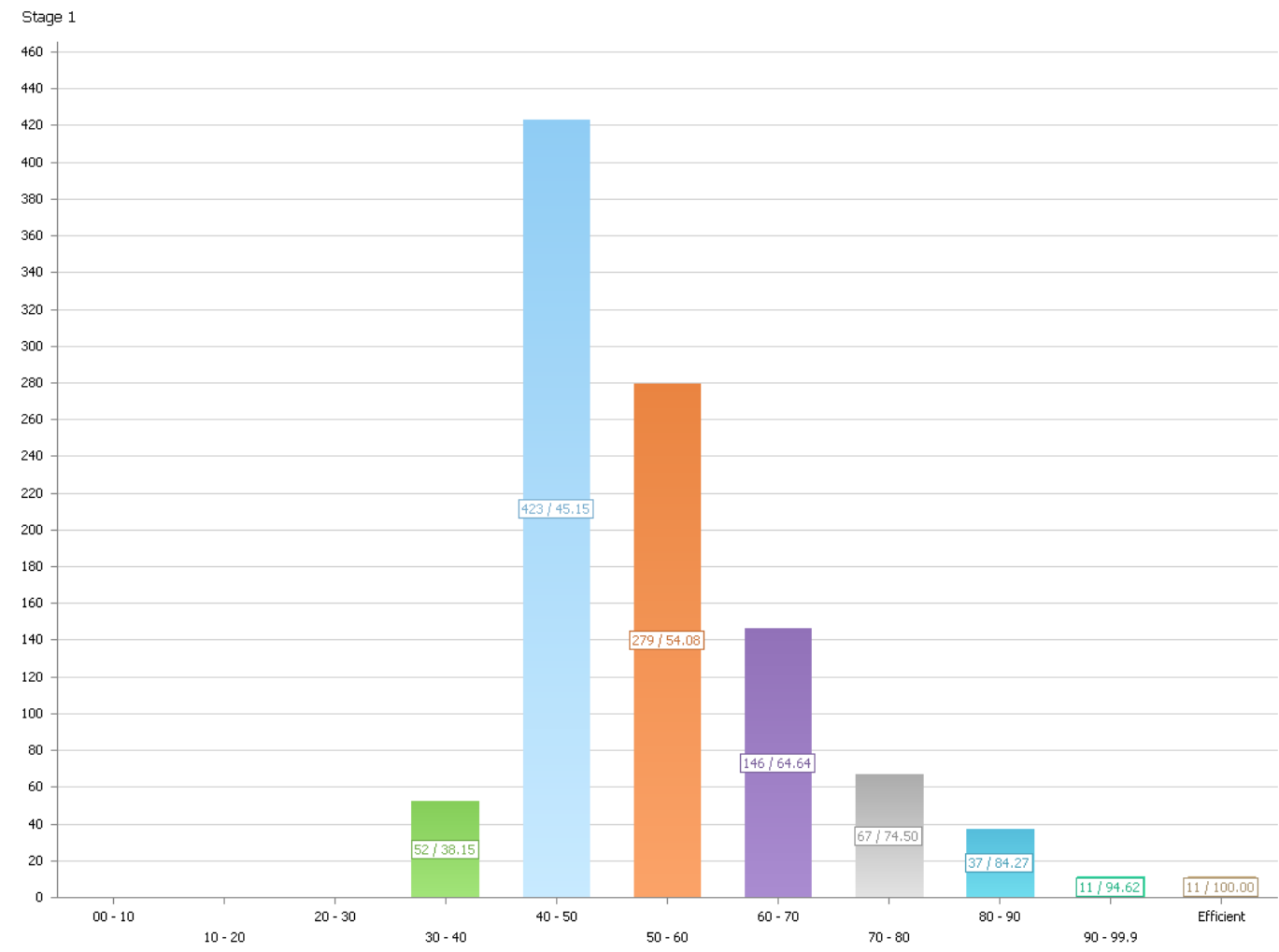

A1.28- Run4

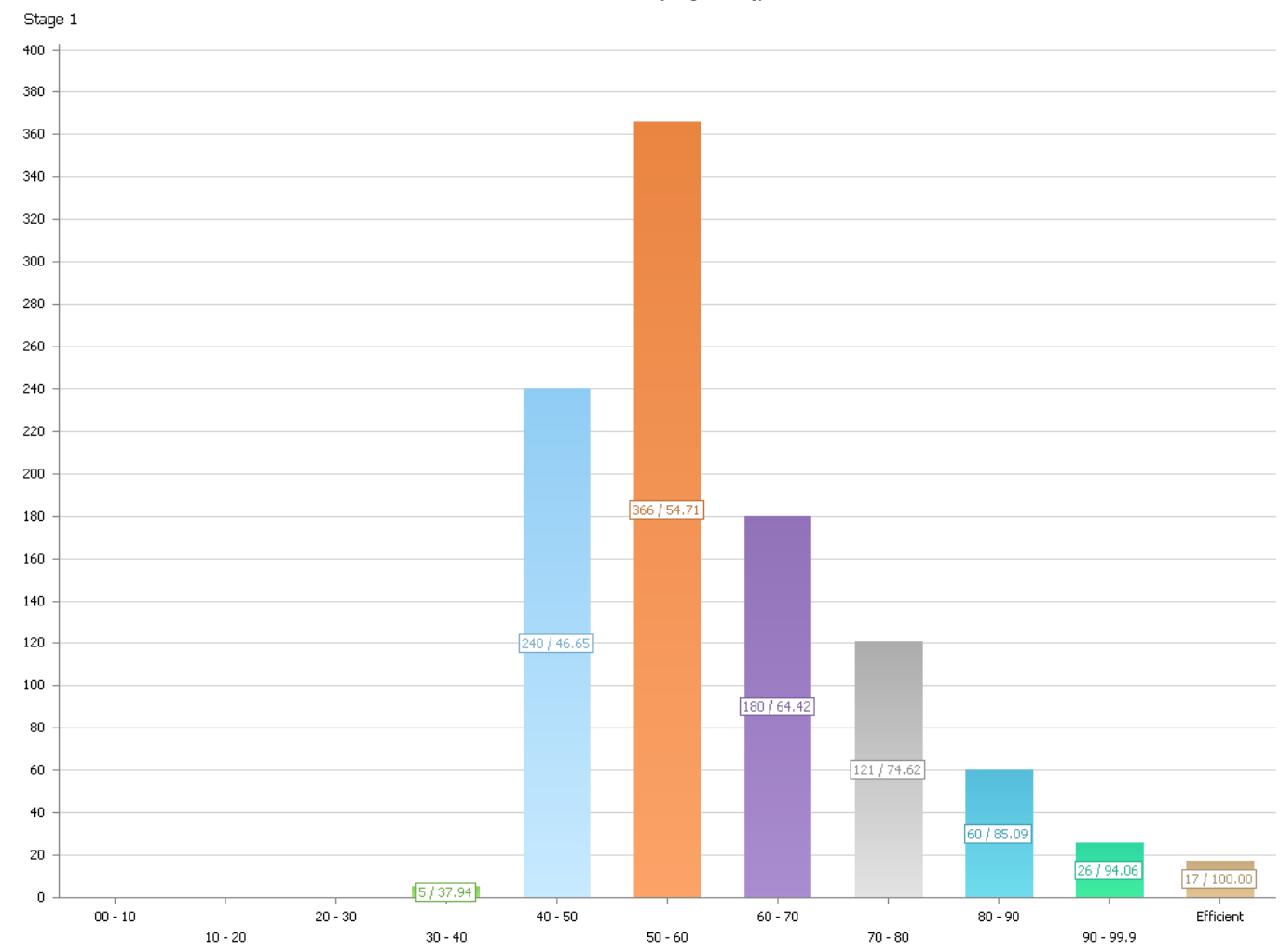




\section{SBM Model}

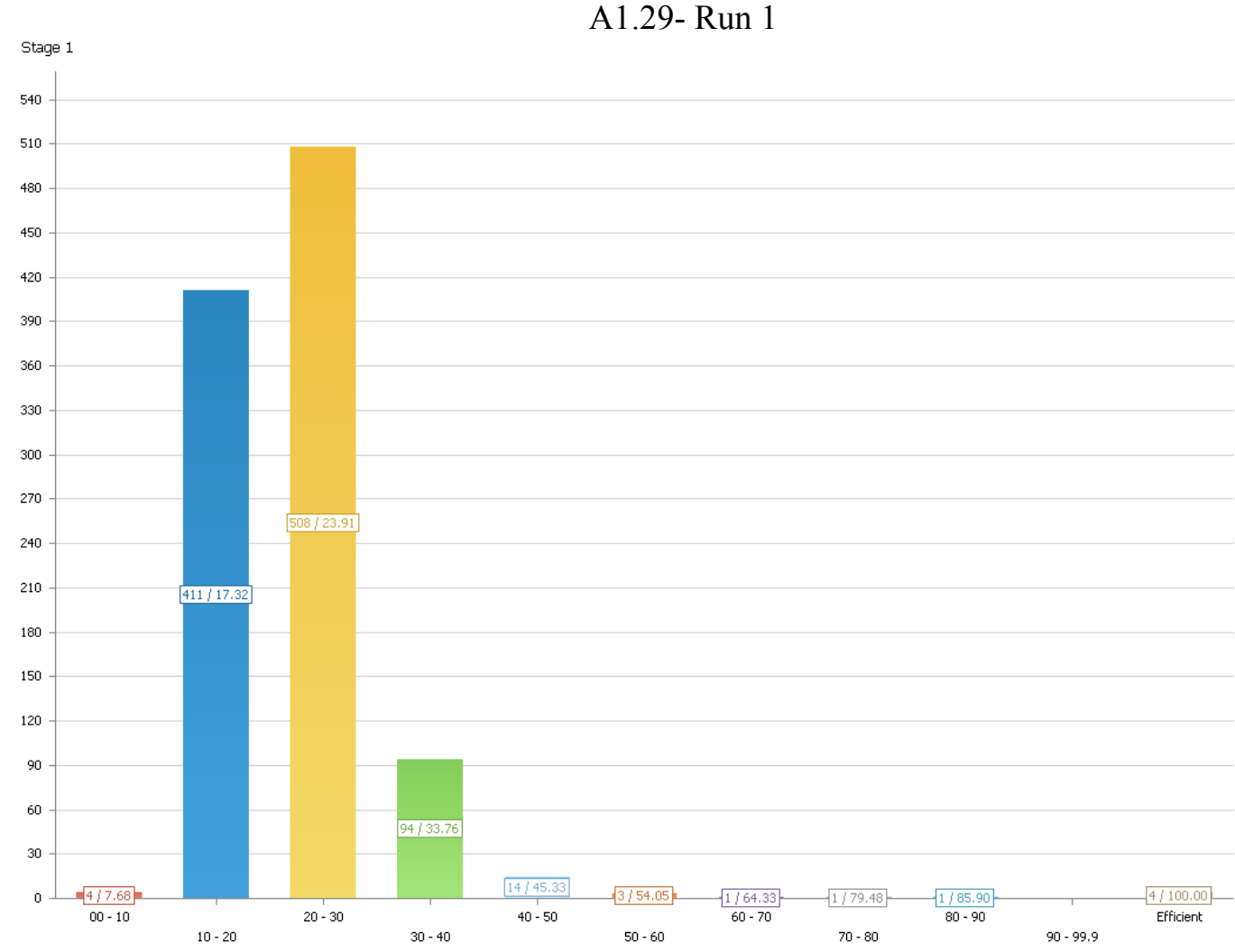

$\checkmark$ (All)

$00-10$
$70-20$

$\checkmark \quad 20-30$

$\checkmark \quad 30-40$

$40-50$
$50-60$

7. $60-70$

1. $70-80$

V.

$\checkmark=90-99.9$

$\checkmark$ Efficient

A1.30- Run 2

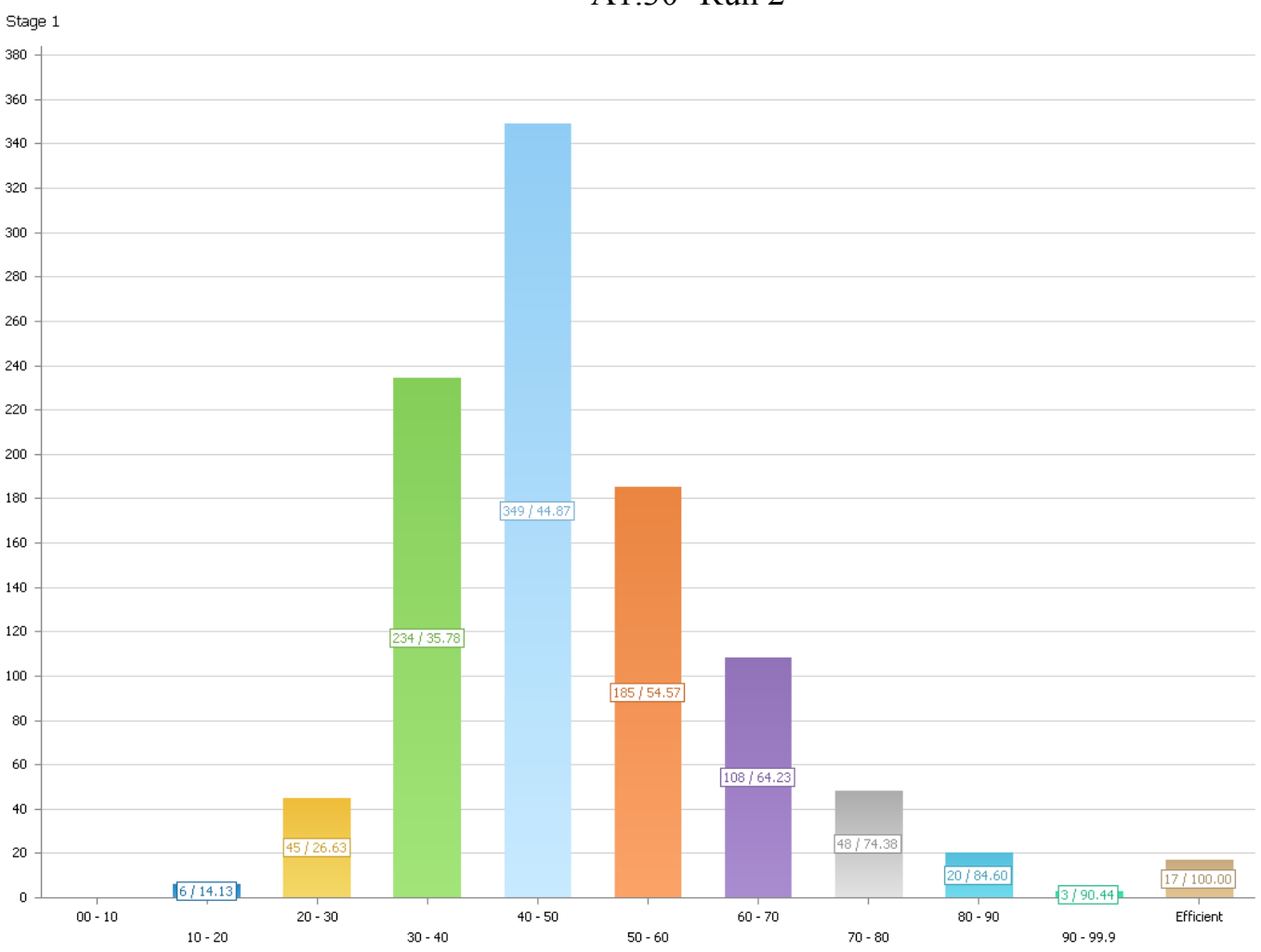




\section{Percent Model}

\section{CCR Model}

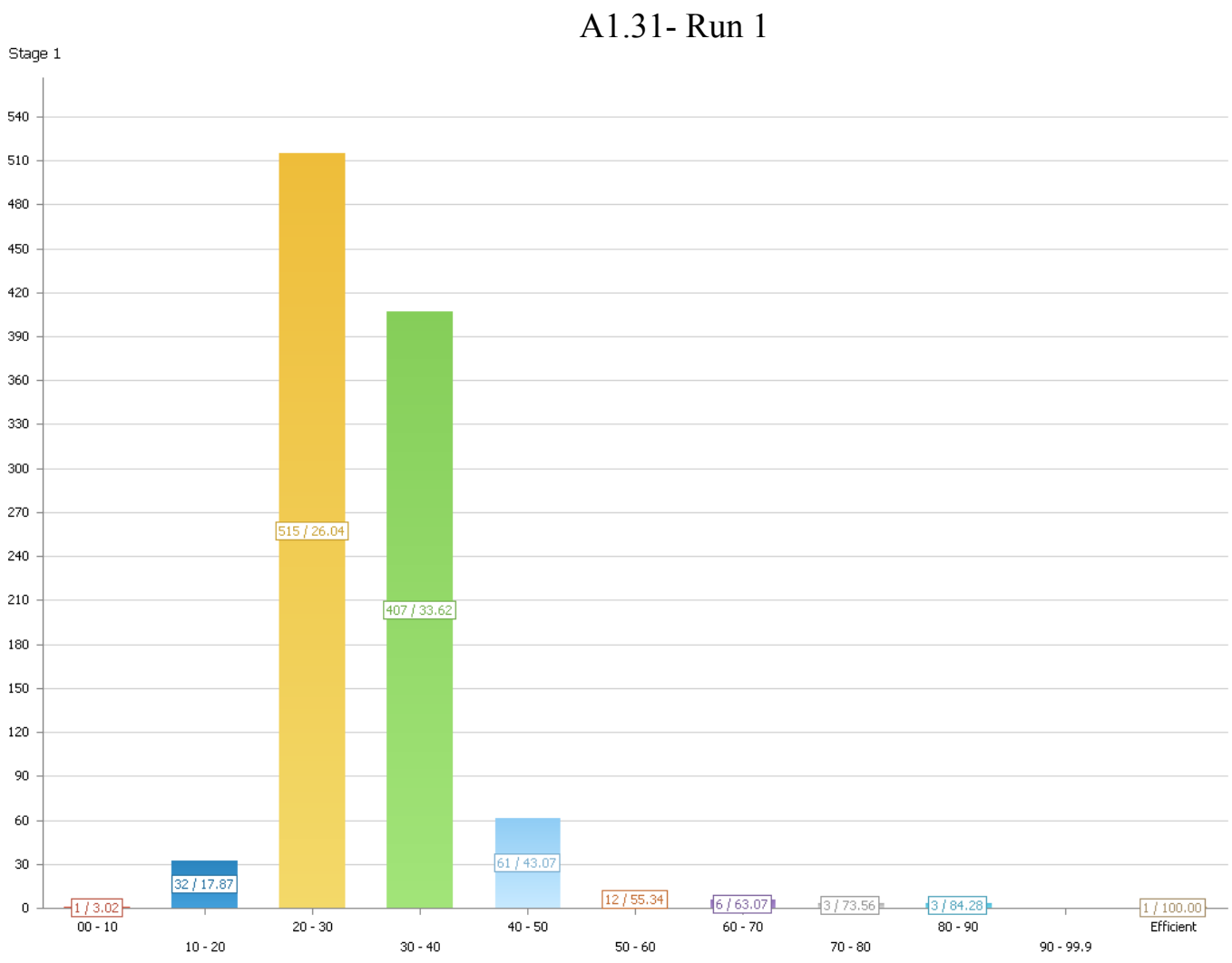

A1.32- Run2

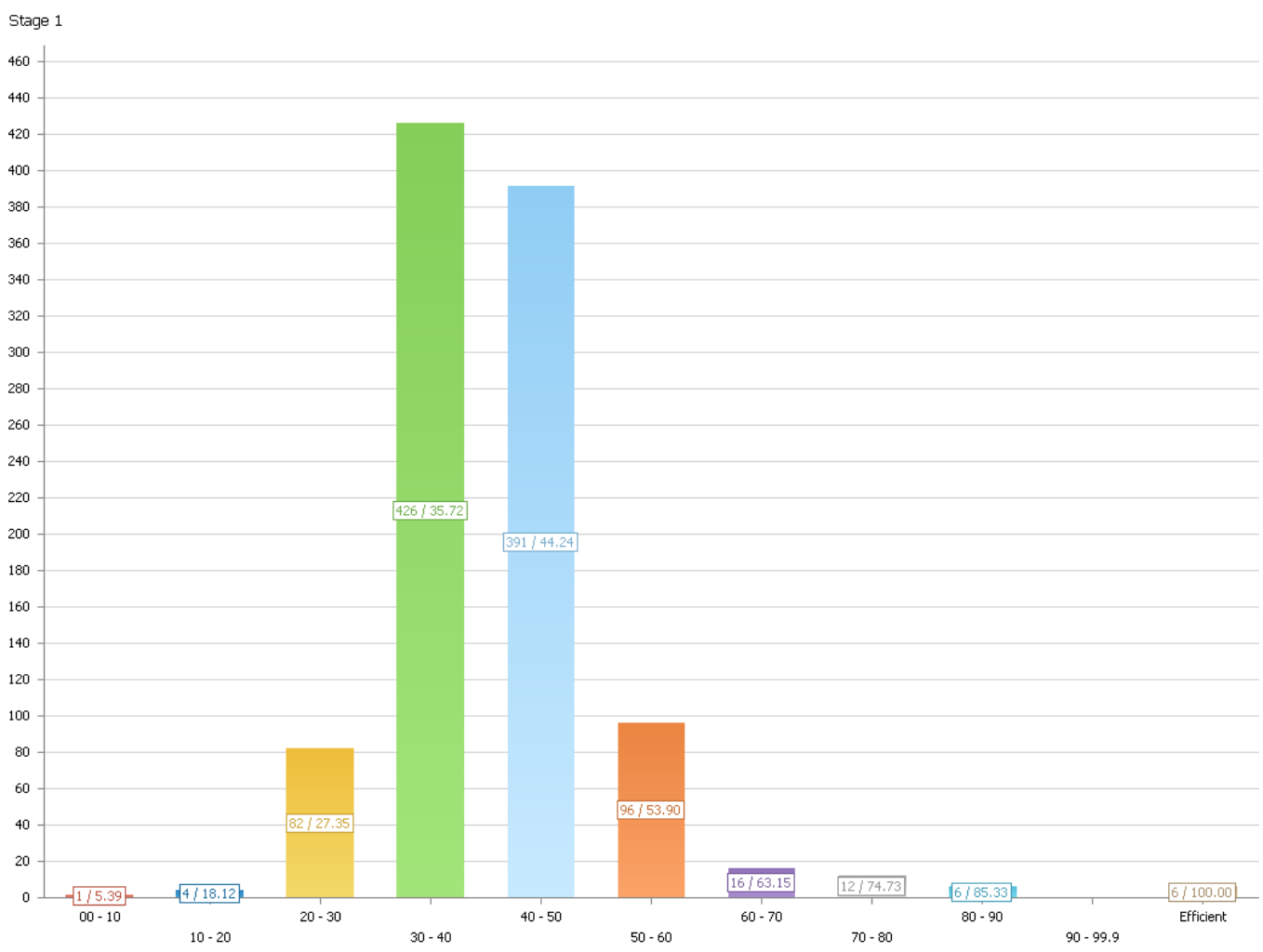


A1.33- Run3

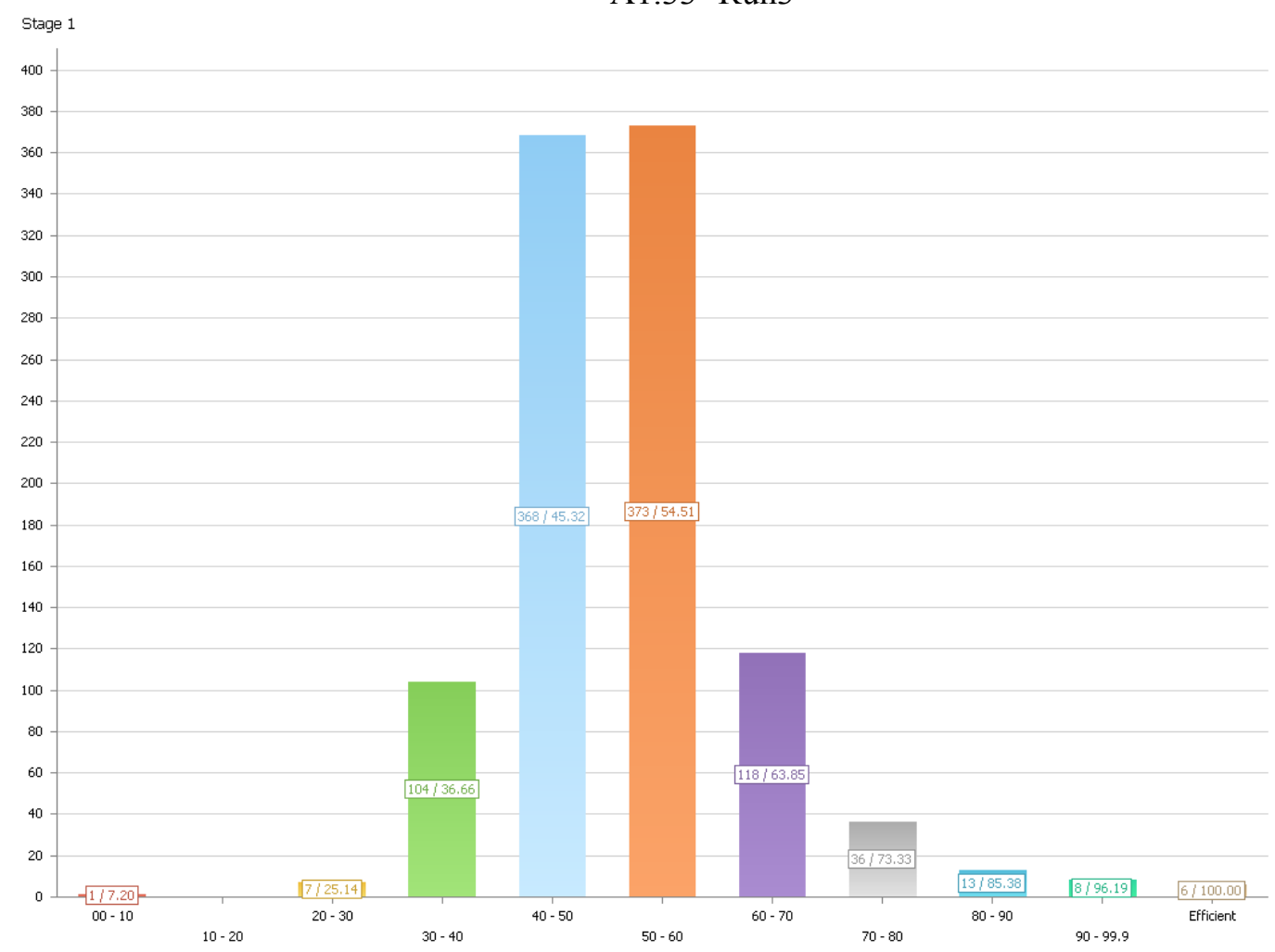

$\checkmark$ (All)

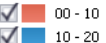

$\checkmark 20-30$

$\checkmark \quad 30-40$

$\checkmark \quad 40-50$

$50-60$

$60-70$
$70-80$

V.

$\checkmark=90-99.9$

$\checkmark$ Efficient

A1.34- Run4

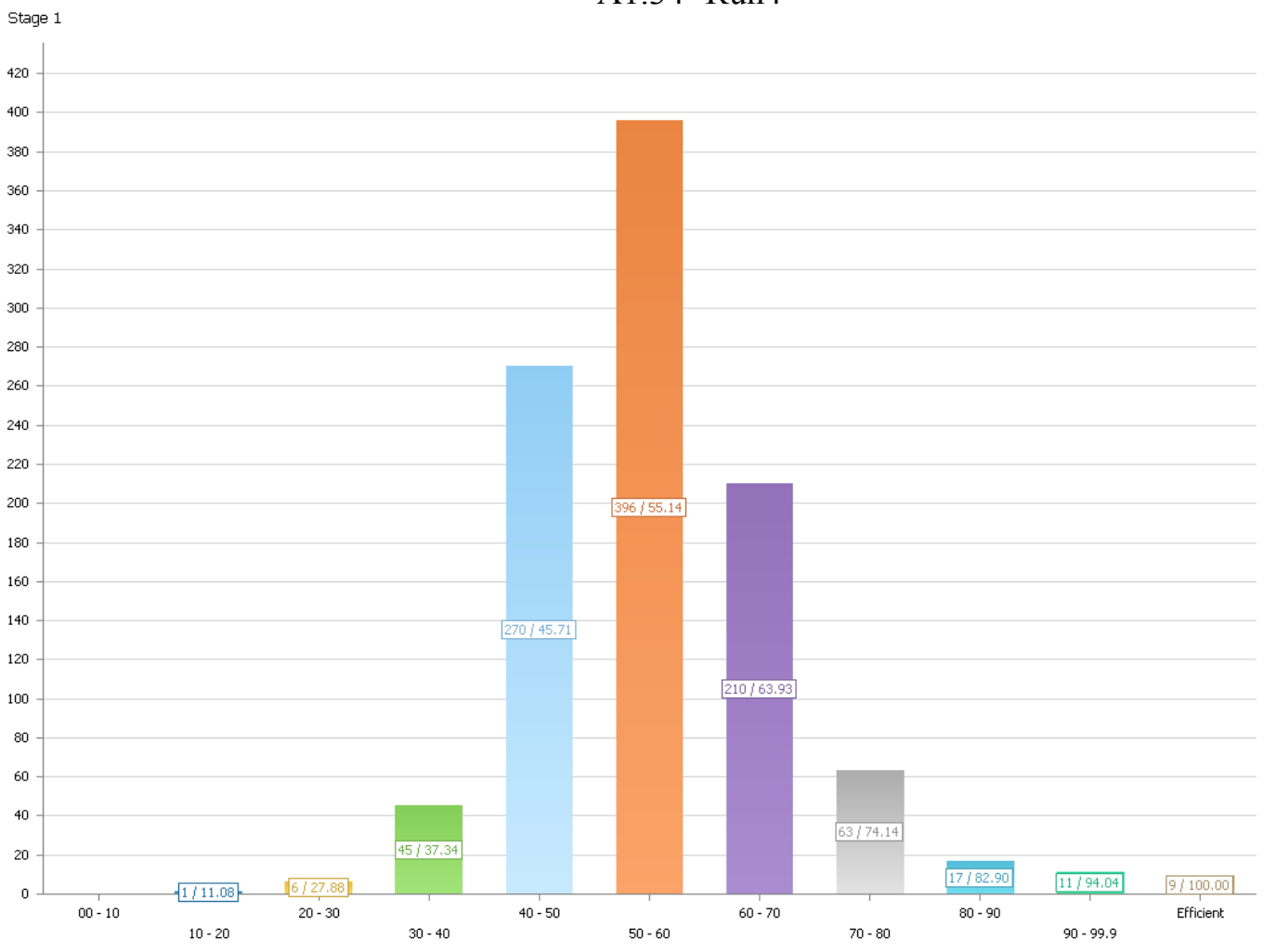




\section{SBM Model}

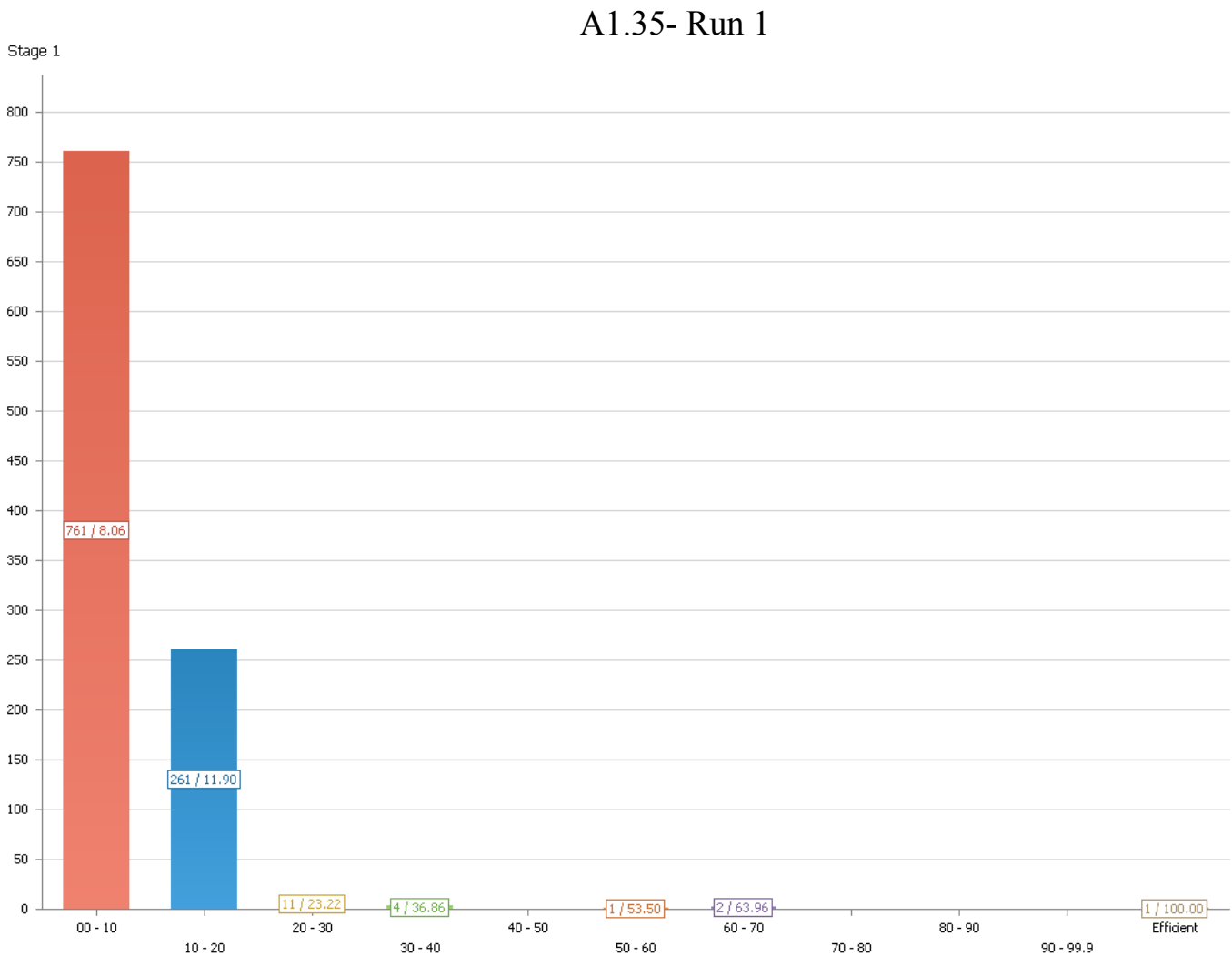

A1.36- Run 2

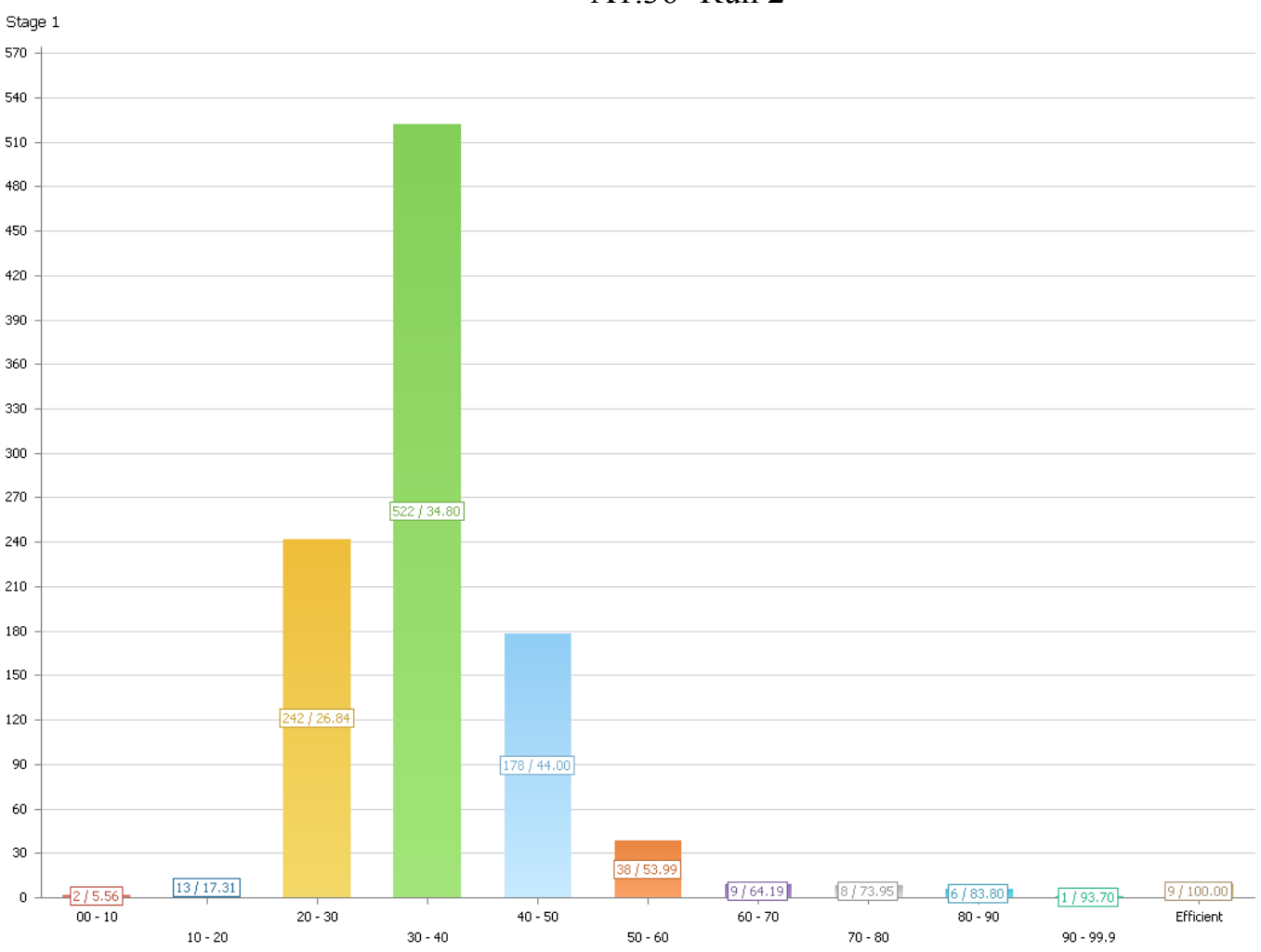




\section{Delta Models: Data Analysis and Segmentation}

\section{Difference Model}

CCR Model

A1.37- Run 1

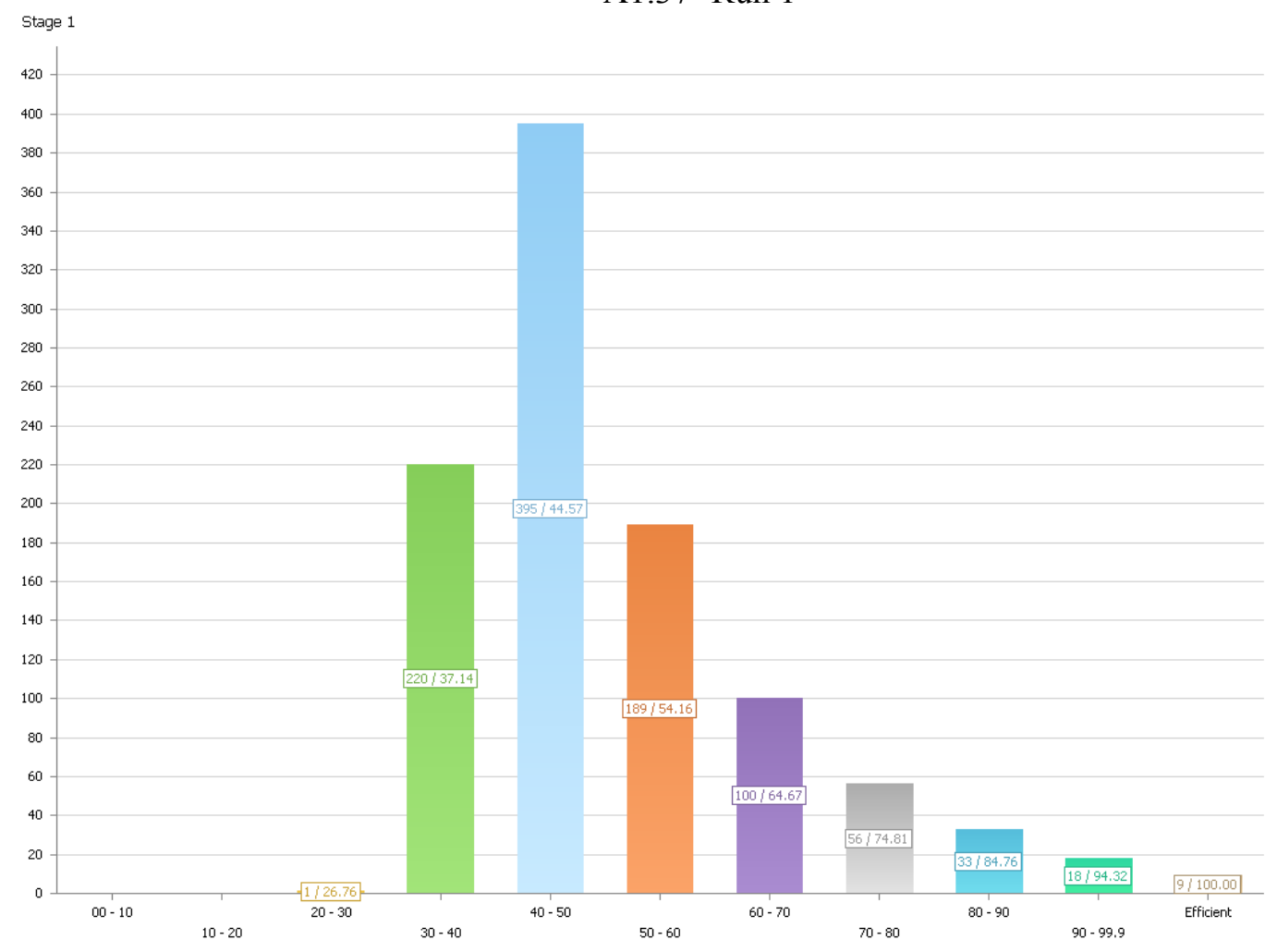

A1.38- Run2

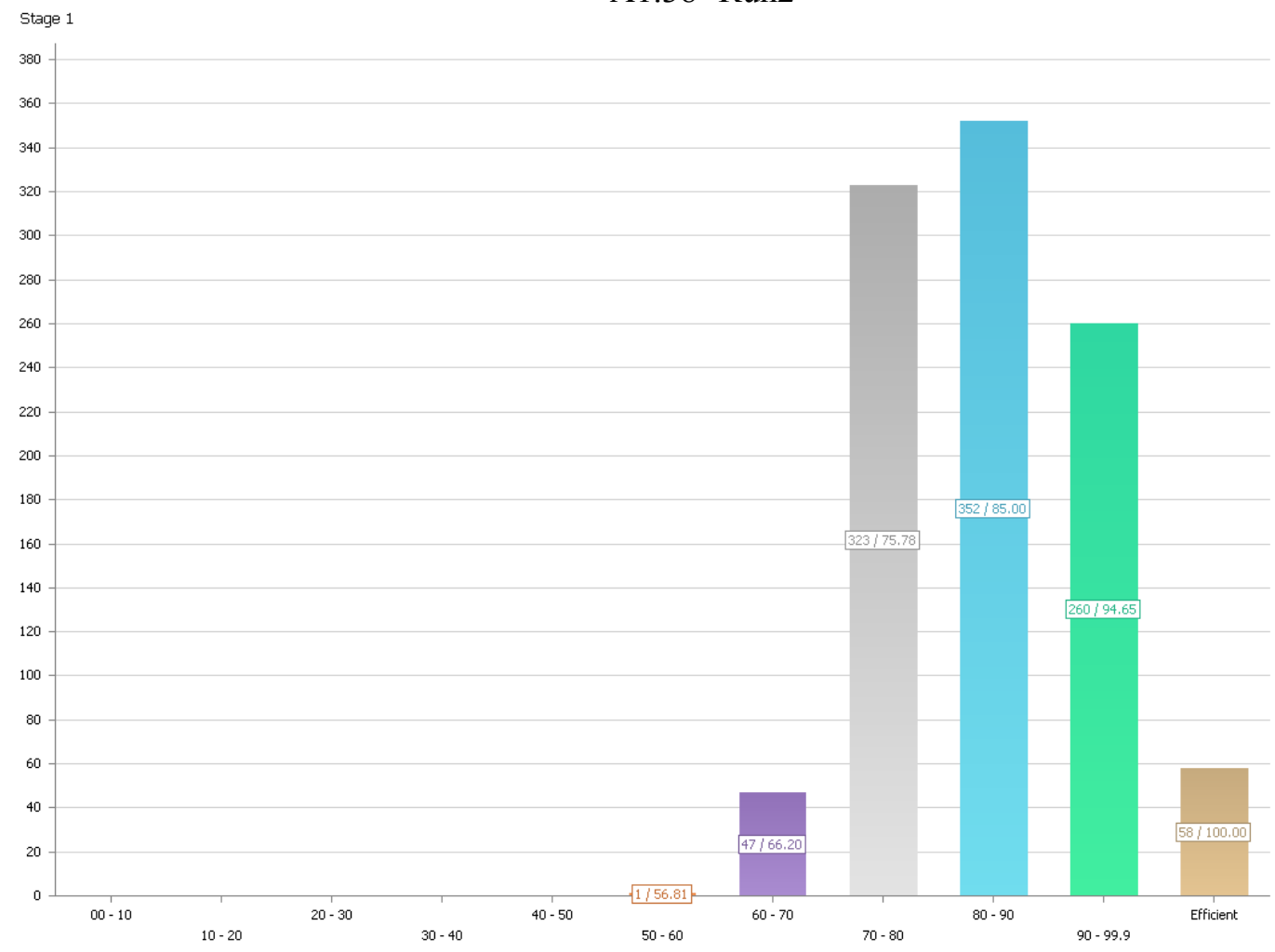


A1.39- Run3

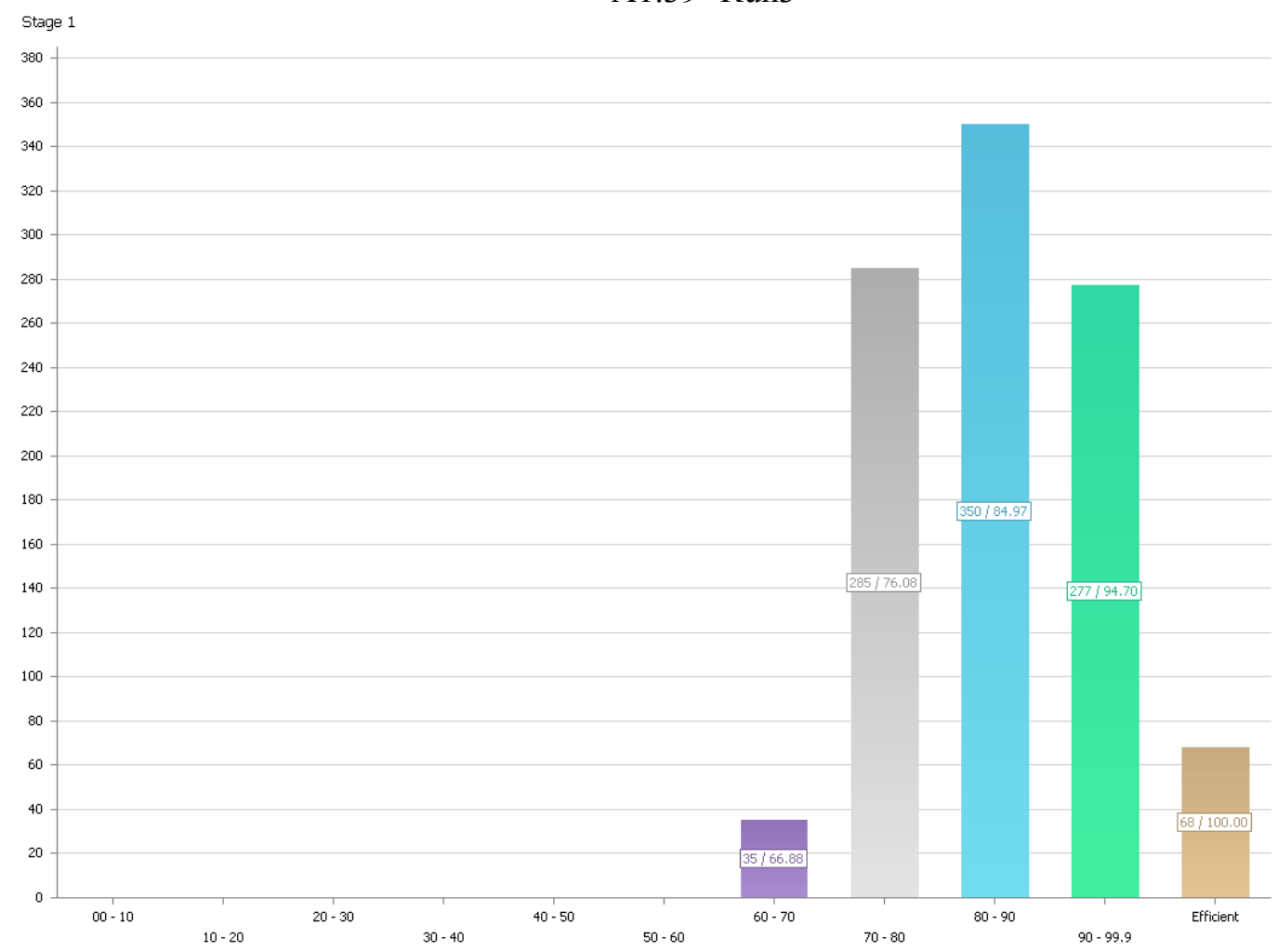

\section{SBM Model}

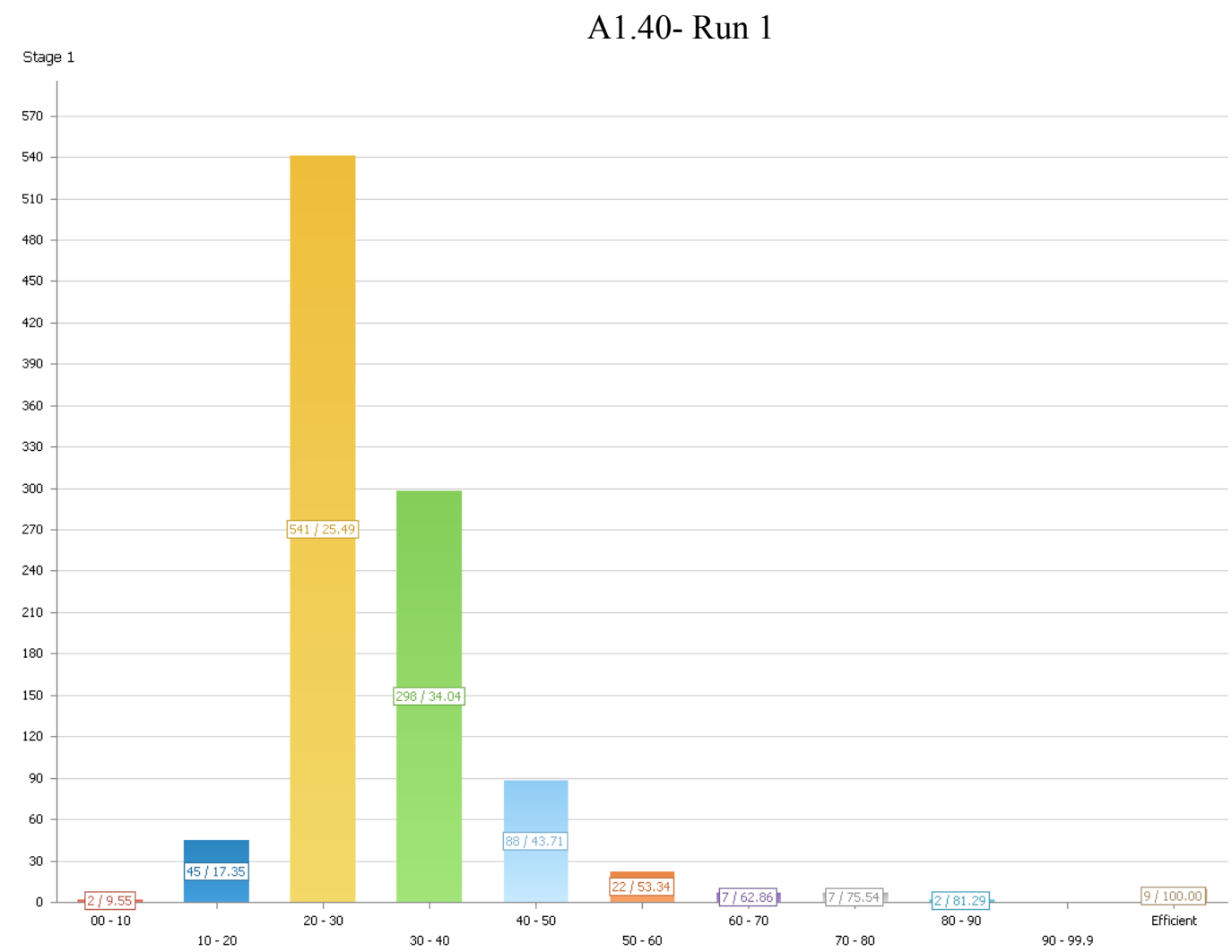


A1.41- Run 2

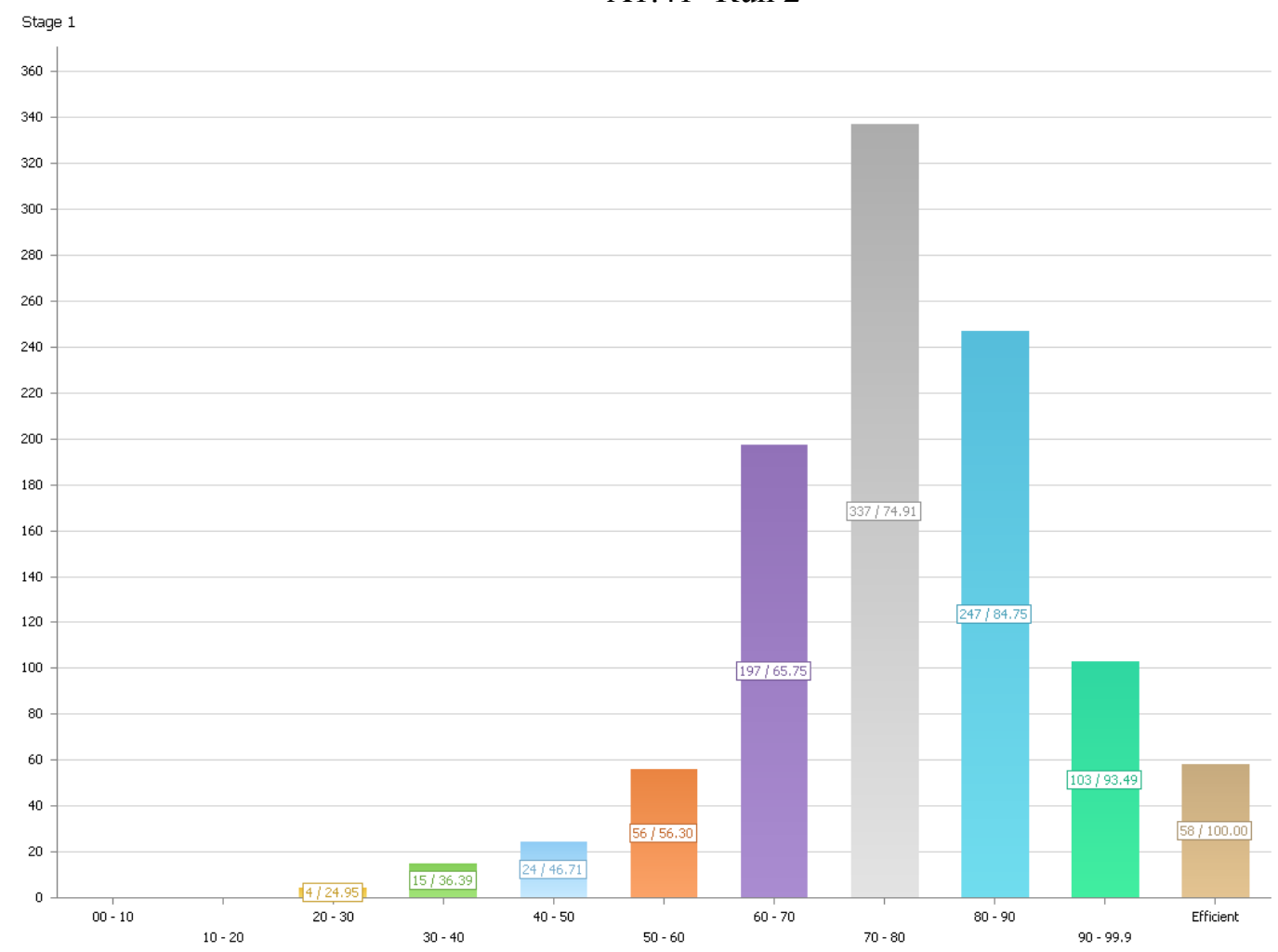

A1.42- Run 3

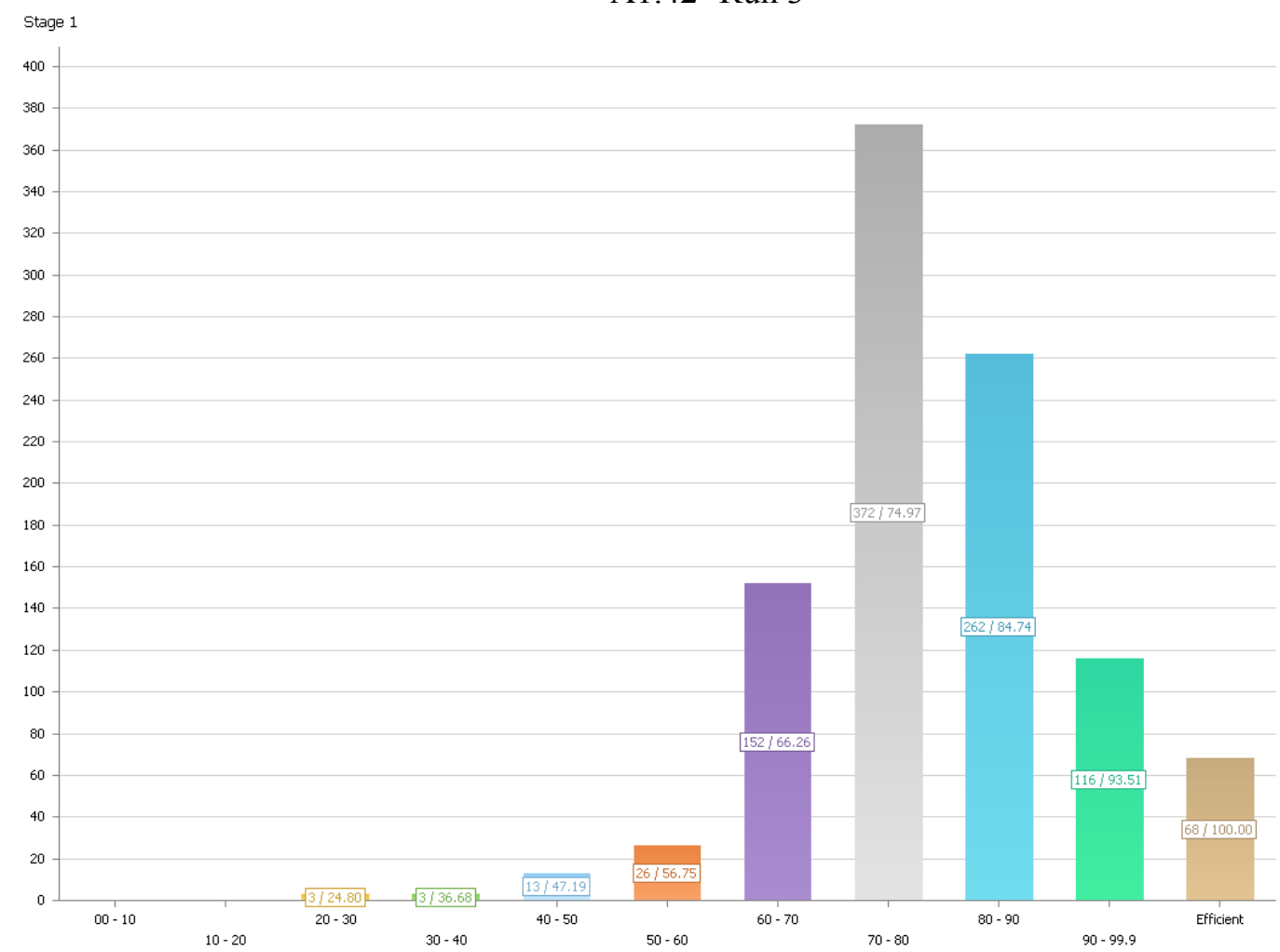




\section{Percent Model}

\section{CCR Model}

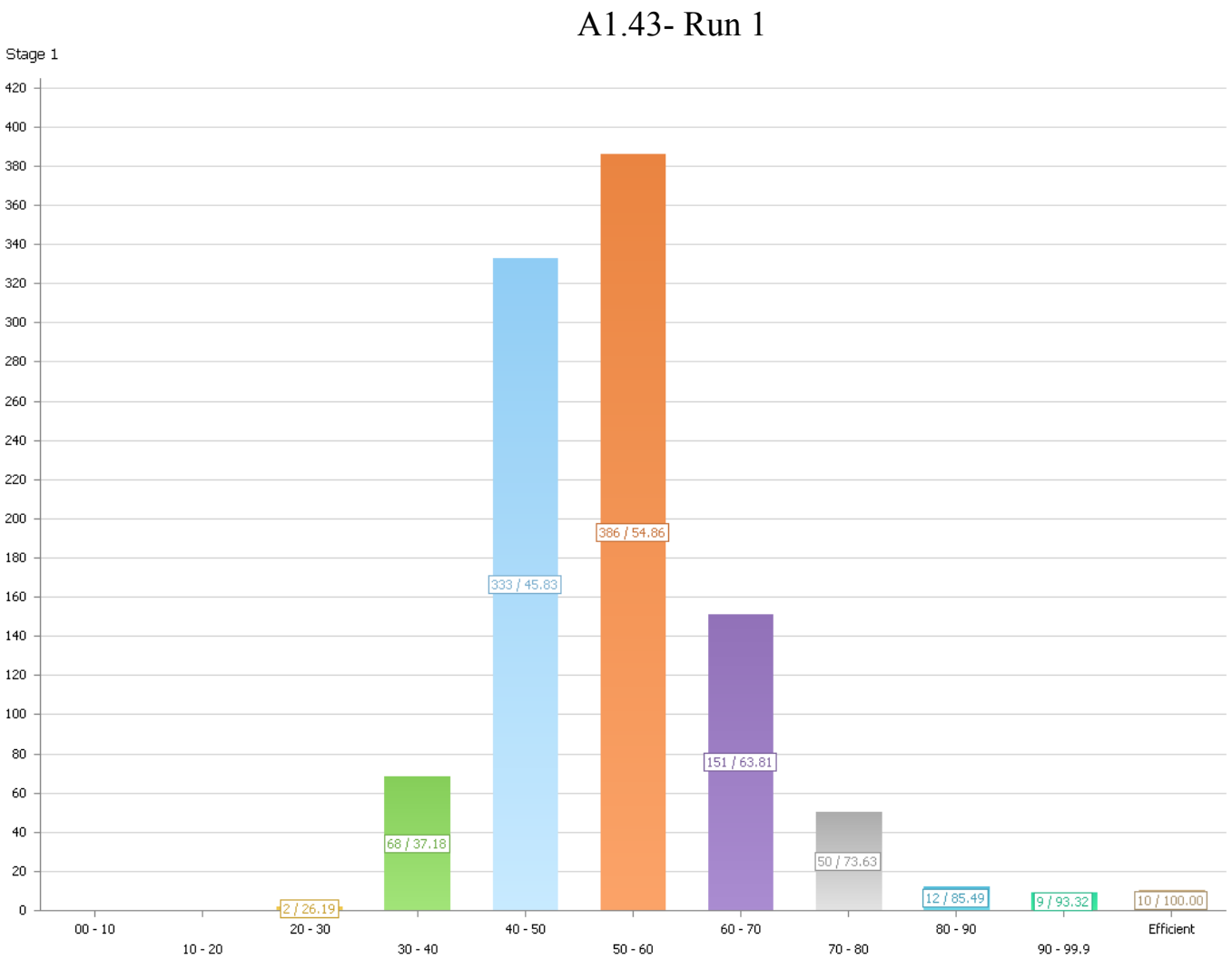

$\checkmark$ (All)

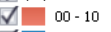

ป $10-20$

1 $20-30$

V. $30-40$

V $\quad 40-50$

V. $50-60$

1. $60-70$

\begin{tabular}{l}
$70-80$ \\
\hline$\quad 80-90$
\end{tabular}

V1 $90-99.9$

$\checkmark$ Efficient

A1.44- Run2

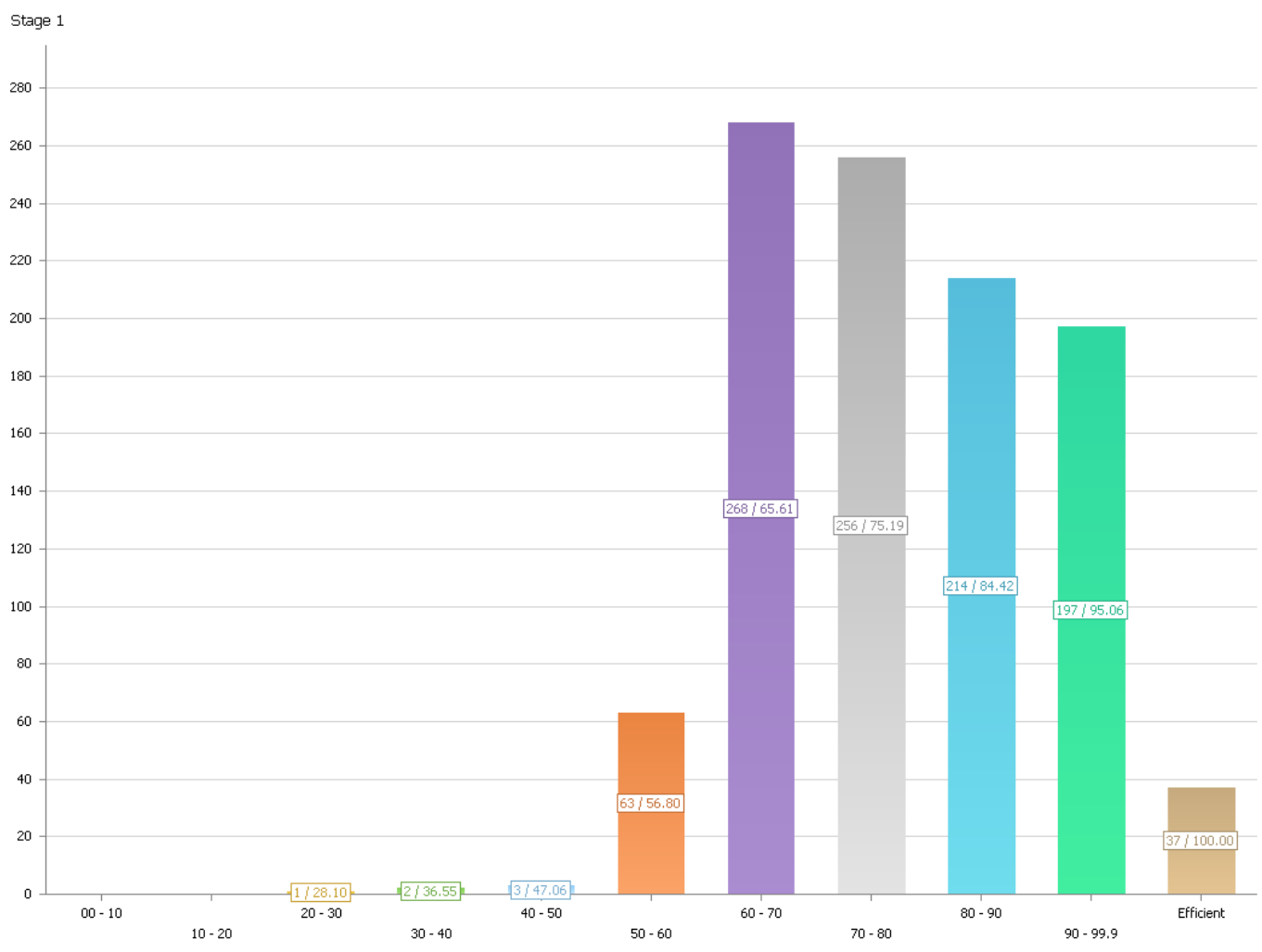


A1.45- Run3

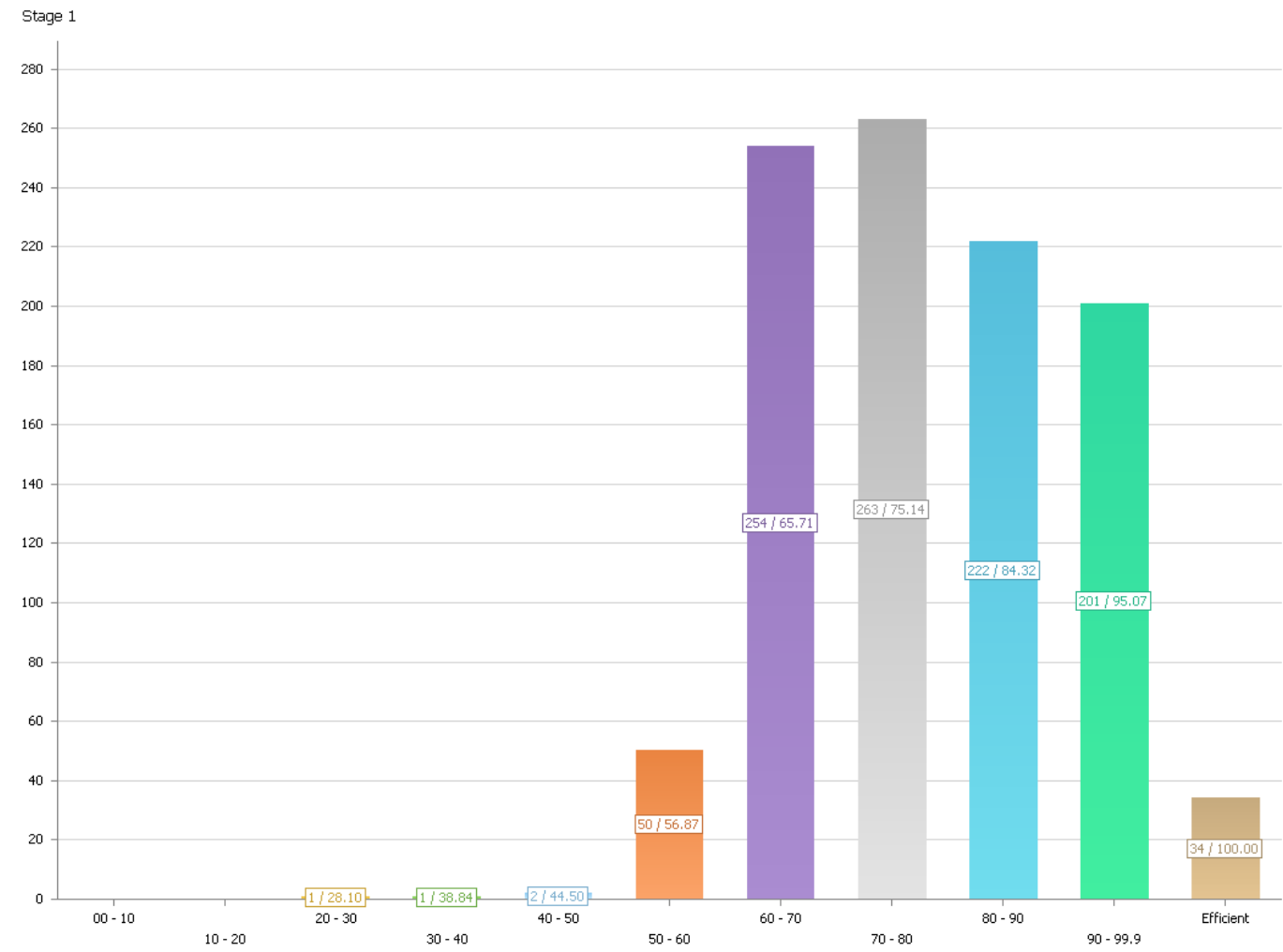

\section{SBM Model}

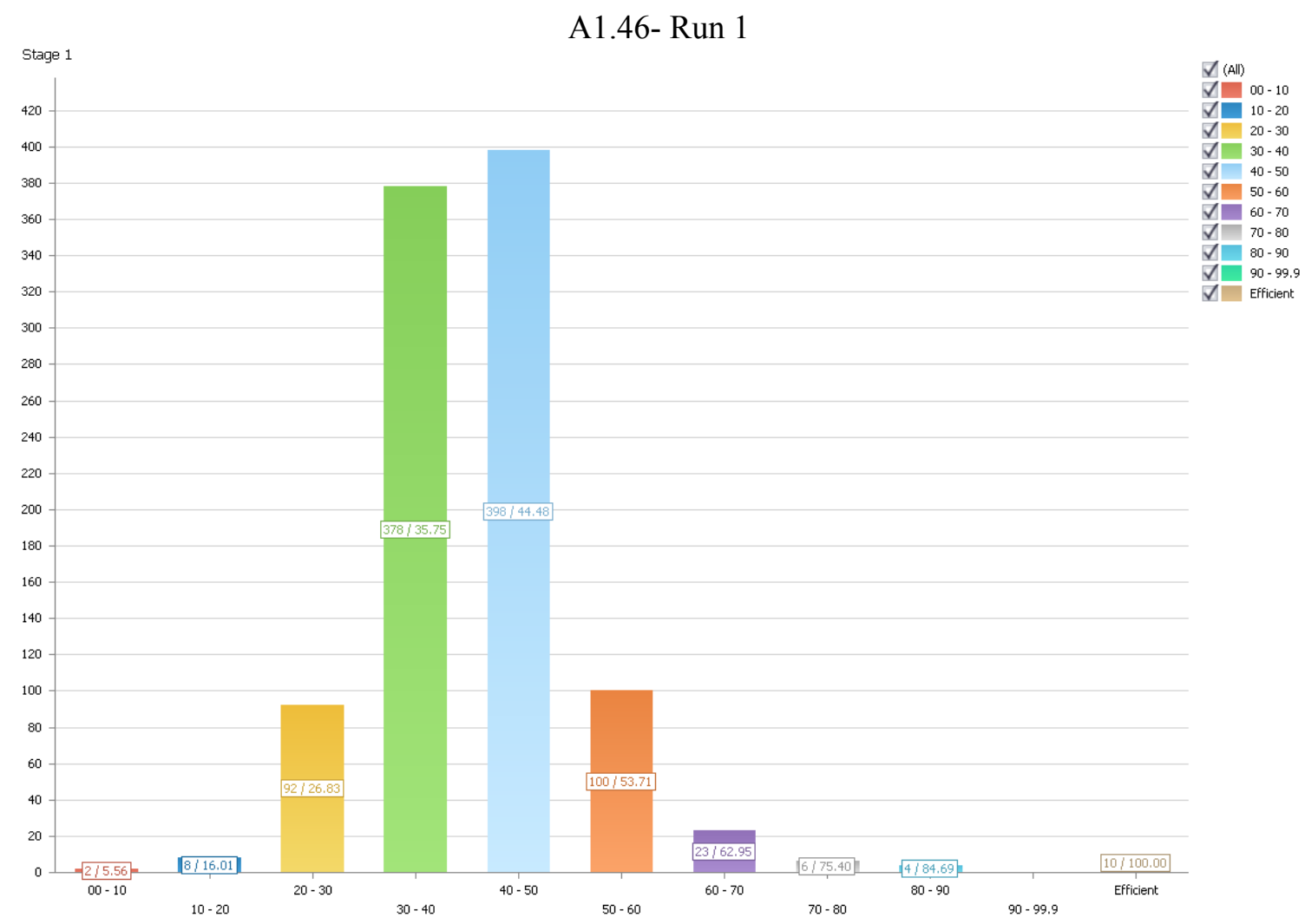




\section{A1.47- Run 2}
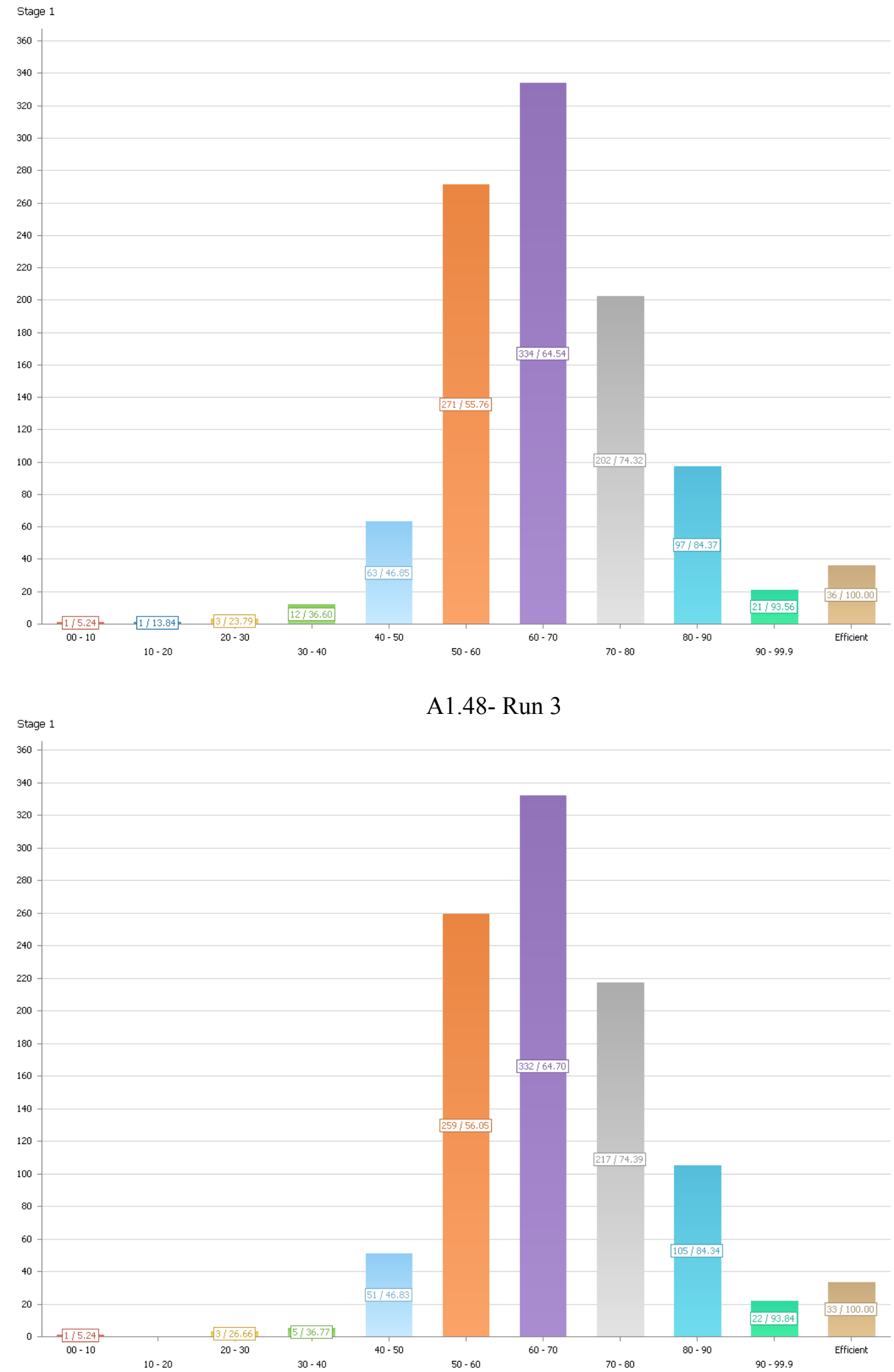


\section{Appendix 2: Segmentation Results Summary}

\section{0-2011Period Growth Model}

Market-

\begin{tabular}{|c|c|c|c|c|c|c|}
\hline \multicolumn{7}{|c|}{ Altlantic Canada } \\
\hline Model/Run & CCR1 & CCR2 & CCR3 & CCR4 & $\mathrm{BCC} 1$ & $\mathrm{BCC} 2$ \\
\hline Average Efficiency & 0.608 & 0.763 & 0.810 & 0.882 & 0.930 & 0.941 \\
\hline Standard Deviation & 0.074 & 0.061 & 0.059 & 0.047 & 0.034 & 0.031 \\
\hline \multicolumn{7}{|c|}{ Quebec } \\
\hline Model/Run & CCR1 & CCR2 & CCR3 & CCR4 & $\mathrm{BCC} 1$ & $\mathrm{BCC} 2$ \\
\hline Average Efficiency & 0.621 & 0.775 & 0.829 & 0.896 & 0.926 & 0.949 \\
\hline Standard Deviation & 0.106 & 0.089 & 0.064 & 0.054 & 0.044 & 0.031 \\
\hline \multicolumn{7}{|c|}{ Northern Ontario } \\
\hline Model/Run & CCR1 & CCR2 & CCR3 & CCR4 & $\mathrm{BCC} 1$ & $\mathrm{BCC2}$ \\
\hline Average Efficiency & 0.630 & 0.783 & 0.833 & 0.899 & 0.933 & 0.950 \\
\hline Standard Deviation & 0.084 & 0.065 & 0.061 & 0.046 & 0.028 & 0.024 \\
\hline \multicolumn{7}{|c|}{ Greater Toronto Area- Central } \\
\hline Model/Run & CCR1 & CCR2 & CCR3 & CCR4 & $\mathrm{BCC} 1$ & $\mathrm{BCC} 2$ \\
\hline Average Efficiency & 0.635 & 0.771 & 0.817 & 0.890 & 0.938 & 0.949 \\
\hline Standard Deviation & 0.084 & 0.062 & 0.059 & 0.045 & 0.033 & 0.028 \\
\hline \multicolumn{7}{|c|}{ Greater Toronto Area- West } \\
\hline Model/Run & CCR1 & CCR2 & CCR3 & CCR4 & $\mathrm{BCC1}$ & $\mathrm{BCC} 2$ \\
\hline Average Efficiency & 0.620 & 0.753 & 0.798 & 0.866 & 0.920 & 0.931 \\
\hline Standard Deviation & 0.091 & 0.066 & 0.058 & 0.034 & 0.043 & 0.029 \\
\hline \multicolumn{7}{|c|}{ Greater Toronto Area- East } \\
\hline Model/Run & CCR1 & CCR2 & CCR3 & CCR4 & $\mathrm{BCC} 1$ & $\mathrm{BCC} 2$ \\
\hline Average Efficiency & 0.608 & 0.762 & 0.811 & 0.887 & 0.934 & 0.946 \\
\hline Standard Deviation & 0.071 & 0.071 & 0.063 & 0.047 & 0.050 & 0.031 \\
\hline \multicolumn{7}{|c|}{ Southern Ontario } \\
\hline Model/Run & CCR1 & CCR2 & CCR3 & CCR4 & $\mathrm{BCC} 1$ & $\mathrm{BCC} 2$ \\
\hline Average Efficiency & 0.669 & 0.800 & 0.835 & 0.903 & 0.904 & 0.937 \\
\hline Standard Deviation & 0.063 & 0.061 & 0.053 & 0.042 & 0.039 & 0.028 \\
\hline \multicolumn{7}{|c|}{ Prairies (Manitoba and Saskatchewan) } \\
\hline Model/Run & CCR1 & CCR2 & CCR3 & CCR4 & $\mathrm{BCC} 1$ & $\mathrm{BCC2}$ \\
\hline Average Efficiency & 0.676 & 0.814 & 0.854 & 0.910 & 0.882 & 0.946 \\
\hline Standard Deviation & 0.093 & 0.074 & 0.073 & 0.048 & 0.082 & 0.039 \\
\hline \multicolumn{7}{|c|}{ Alberta } \\
\hline Model/Run & CCR1 & CCR2 & CCR3 & CCR4 & $\mathrm{BCCl}$ & $\mathrm{BCC} 2$ \\
\hline Average Efficiency & 0.589 & 0.761 & 0.814 & 0.885 & 0.916 & 0.937 \\
\hline Standard Deviation & 0.086 & 0.065 & 0.056 & 0.045 & 0.049 & 0.036 \\
\hline \multicolumn{7}{|c|}{ Vancouver Lower Mainland } \\
\hline Model/Run & CCR1 & CCR2 & CCR3 & CCR4 & $\mathrm{BCCl}$ & $\mathrm{BCC} 2$ \\
\hline Average Efficiency & 0.590 & 0.750 & 0.813 & 0.886 & 0.936 & 0.946 \\
\hline Standard Deviation & 0.078 & 0.060 & 0.064 & 0.049 & 0.035 & 0.031 \\
\hline \multicolumn{7}{|c|}{ Vancouver Island, Central BC, Northern Territories } \\
\hline Model/Run & CCR1 & CCR2 & CCR3 & CCR4 & $\mathrm{BCC} 1$ & $\mathrm{BCC2}$ \\
\hline Average Efficiency & 0.609 & 0.764 & 0.812 & 0.886 & 0.922 & 0.938 \\
\hline Standard Deviation & 0.079 & 0.059 & 0.056 & 0.045 & 0.032 & 0.026 \\
\hline
\end{tabular}

Urban/Rural-

\begin{tabular}{|l|c|c|c|c|c|c|}
\hline \hline \multicolumn{7}{|c|}{ Urban } \\
\hline Model/Run & CCR1 & CCR2 & CCR3 & CCR4 & BCC1 & BCC2 \\
\hline Average Efficiency & 0.595 & 0.751 & 0.806 & 0.879 & 0.933 & 0.943 \\
\hline Standard Deviation & 0.080 & 0.059 & 0.054 & 0.043 & 0.037 & 0.031 \\
\hline \multicolumn{7}{|l|}{ Rural } \\
\hline Model/Run & CCR1 & CCR2 & CCR3 & CCR4 & BCC1 & BCC2 \\
\hline Average Efficiency & 0.658 & 0.801 & 0.843 & 0.906 & 0.906 & 0.943 \\
\hline Standard Deviation & 0.087 & 0.069 & 0.064 & 0.047 & 0.055 & 0.032 \\
\hline \hline
\end{tabular}

Multi-Stage Evaluation of Bank Branch Consolidation and Growth Potential Using DEA 134 
Funds Managed-

\begin{tabular}{|c|c|c|c|c|c|c|}
\hline \multicolumn{7}{|c|}{$3,313,956.60-80,282,296.80$} \\
\hline Model/Run & CCR1 & CCR2 & CCR3 & CCR4 & BCC1 & $\mathrm{BCC} 2$ \\
\hline Average Efficiency & 0.657 & 0.793 & 0.838 & 0.901 & 0.896 & 0.935 \\
\hline Standard Deviation & 0.097 & 0.079 & 0.070 & 0.053 & 0.053 & 0.036 \\
\hline \multicolumn{7}{|c|}{$80,481,614.72-170,379,805.97$} \\
\hline Model/Run & CCR1 & CCR2 & CCR3 & CCR4 & BCC1 & $\mathrm{BCC} 2$ \\
\hline Average Efficiency & 0.591 & 0.753 & 0.805 & 0.880 & 0.938 & 0.945 \\
\hline Standard Deviation & 0.063 & 0.050 & 0.045 & 0.037 & 0.022 & 0.022 \\
\hline \multicolumn{7}{|c|}{$171,200,464.57-336,017,752.75$} \\
\hline Model/Run & CCR1 & CCR2 & CCR3 & CCR4 & BCC1 & $\mathrm{BCC} 2$ \\
\hline Average Efficiency & 0.589 & 0.764 & 0.816 & 0.889 & 0.965 & 0.967 \\
\hline Standard Deviation & 0.064 & 0.052 & 0.050 & 0.040 & 0.018 & 0.019 \\
\hline \multicolumn{7}{|c|}{$359,066,886.41-1,165,724,792.51$} \\
\hline Model/Run & CCR1 & CCR2 & CCR3 & CCR4 & BCC1 & $\mathrm{BCC} 2$ \\
\hline Average Efficiency & 0.609 & 0.791 & 0.834 & 0.897 & 0.982 & 0.981 \\
\hline Standard Deviation & 0.121 & 0.081 & 0.063 & 0.047 & 0.019 & 0.019 \\
\hline
\end{tabular}

Existing Customer Base-

\begin{tabular}{|c|c|c|c|c|c|c|}
\hline \hline \multicolumn{7}{|c|}{$342-4,092$ Customers } \\
\hline Model/Run & CCR1 & CCR2 & CCR3 & CCR4 & BCC1 & BCC2 \\
\hline Average Efficiency & 0.669 & 0.809 & 0.852 & 0.912 & 0.890 & 0.937 \\
\hline Standard Deviation & 0.098 & 0.077 & 0.070 & 0.051 & 0.057 & 0.038 \\
\hline \multicolumn{7}{|c|}{$4,117-8,038$ Customers } \\
\hline Model/Run & CCR1 & CCR2 & CCR3 & CCR4 & BCC1 & BCC2 \\
\hline Average Efficiency & 0.596 & 0.752 & 0.804 & 0.879 & 0.929 & 0.939 \\
\hline Standard Deviation & 0.066 & 0.053 & 0.048 & 0.040 & 0.025 & 0.024 \\
\hline \multicolumn{7}{|c|}{$8,186-14,314$ Customers } \\
\hline Model/Run & CCR1 & CCR2 & CCR3 & CCR4 & BCC1 & BCC2 \\
\hline Average Efficiency & 0.593 & 0.752 & 0.803 & 0.878 & 0.955 & 0.958 \\
\hline Standard Deviation & 0.074 & 0.055 & 0.046 & 0.039 & 0.020 & 0.021 \\
\hline Model/Run & $14,524-37,198$ Customers & & \\
\hline Average Efficiency & CCR1 & CCR2 & CCR3 & CCR4 & BCC1 & BCC2 \\
\hline Standard Deviation & 0.597 & 0.766 & 0.814 & 0.888 & 0.975 & 0.975 \\
\hline
\end{tabular}

Returns to Scale-

\begin{tabular}{|c|c|c|c|c|c|c|}
\hline \hline \multicolumn{7}{|c|}{ All DMUs } \\
\hline Model/Run & CCR1 & CCR2 & CCR3 & CCR4 & BCC1 & BCC2 \\
\hline CRS & $100.0 \%$ & $100.0 \%$ & $100.0 \%$ & $100.0 \%$ & $0.1 \%$ & $0.3 \%$ \\
\hline IRS & $0.0 \%$ & $0.0 \%$ & $0.0 \%$ & $0.0 \%$ & $0.4 \%$ & $6.7 \%$ \\
\hline DRS & $0.0 \%$ & $0.0 \%$ & $0.0 \%$ & $0.0 \%$ & $99.5 \%$ & $93.0 \%$ \\
\hline \multicolumn{7}{|c|}{ Funds-S } \\
\hline Model/Run & CCR1 & CCR2 & CCR3 & CCR4 & BCC1 & BCC2 \\
\hline CRS & $100.0 \%$ & $100.0 \%$ & $100.0 \%$ & $100.0 \%$ & $0.2 \%$ & $0.4 \%$ \\
\hline IRS & $0.0 \%$ & $0.0 \%$ & $0.0 \%$ & $0.0 \%$ & $0.8 \%$ & $13.2 \%$ \\
\hline DRS & $0.0 \%$ & $0.0 \%$ & $0.0 \%$ & $0.0 \%$ & $99.0 \%$ & $86.4 \%$ \\
\hline \multicolumn{7}{|c|}{ Funds-M } \\
\hline Model/Run & CCR1 & CCR2 & CCR3 & CCR4 & BCC1 & BCC2 \\
\hline CRS & $100.0 \%$ & $100.0 \%$ & $100.0 \%$ & $100.0 \%$ & $0.0 \%$ & $0.0 \%$ \\
\hline IRS & $0.0 \%$ & $0.0 \%$ & $0.0 \%$ & $0.0 \%$ & $0.0 \%$ & $0.5 \%$ \\
\hline DRS & $0.0 \%$ & $0.0 \%$ & $0.0 \%$ & $0.0 \%$ & $100.0 \%$ & $99.5 \%$ \\
\hline \multicolumn{7}{|c|}{ Funds-L } \\
\hline Model/Run & CCR1 & CCR2 & CCR3 & CCR4 & BCC1 & BCC2 \\
\hline CRS & $100.0 \%$ & $100.0 \%$ & $100.0 \%$ & $100.0 \%$ & $0.0 \%$ & $0.9 \%$ \\
\hline IRS & $0.0 \%$ & $0.0 \%$ & $0.0 \%$ & $0.0 \%$ & $0.0 \%$ & $0.9 \%$ \\
\hline DRS & $0.0 \%$ & $0.0 \%$ & $0.0 \%$ & $0.0 \%$ & $100.0 \%$ & $98.3 \%$ \\
\hline \multicolumn{7}{|c|}{ Funds-XL } \\
\hline Model/Run & CCR1 & CCR2 & CCR3 & CCR4 & BCC1 & BCC2 \\
\hline CRS & $100.0 \%$ & $100.0 \%$ & $100.0 \%$ & $100.0 \%$ & $0.0 \%$ & $0.0 \%$ \\
\hline IRS & $0.0 \%$ & $0.0 \%$ & $0.0 \%$ & $0.0 \%$ & $0.0 \%$ & $0.0 \%$ \\
\hline DRS & $0.0 \%$ & $0.0 \%$ & $0.0 \%$ & $0.0 \%$ & $100.0 \%$ & $100.0 \%$ \\
\hline
\end{tabular}


Lost Accounts/Gained Accounts Model

Market-

\begin{tabular}{|c|c|c|c|c|c|c|}
\hline \multicolumn{7}{|c|}{ Altlantic Canada } \\
\hline Model/Run & CCR1 & CCR2 & CCR3 & CCR4 & BCC1 & $\mathrm{BCC} 2$ \\
\hline Average Efficiency & 0.535 & 0.585 & 0.655 & 0.693 & 0.757 & 0.783 \\
\hline Standard Deviation & 0.105 & 0.121 & 0.129 & 0.114 & 0.118 & 0.118 \\
\hline \multicolumn{7}{|c|}{ Quebec } \\
\hline Model/Run & CCR1 & CCR2 & CCR3 & CCR4 & $\mathrm{BCC1}$ & BCC2 \\
\hline Average Efficiency & 0.520 & 0.602 & 0.669 & 0.735 & 0.741 & 0.801 \\
\hline Standard Deviation & 0.108 & 0.138 & 0.128 & 0.121 & 0.120 & 0.119 \\
\hline \multicolumn{7}{|c|}{ Northern Ontario } \\
\hline Model/Run & CCR1 & CCR2 & CCR3 & CCR4 & $\mathrm{BCC1}$ & BCC2 \\
\hline Average Efficiency & 0.573 & 0.637 & 0.700 & 0.760 & 0.796 & 0.842 \\
\hline Standard Deviation & 0.107 & 0.119 & 0.125 & 0.130 & 0.116 & 0.116 \\
\hline \multicolumn{7}{|c|}{ Greater Toronto Area- Central } \\
\hline Model/Run & CCR1 & CCR2 & CCR3 & CCR4 & $\mathrm{BCC1}$ & $\mathrm{BCC} 2$ \\
\hline Average Efficiency & 0.511 & 0.566 & 0.636 & 0.702 & 0.798 & 0.828 \\
\hline Standard Deviation & 0.076 & 0.097 & 0.100 & 0.097 & 0.101 & 0.098 \\
\hline \multicolumn{7}{|c|}{ Greater Toronto Area- West } \\
\hline Model/Run & CCR1 & CCR2 & CCR3 & CCR4 & $\mathrm{BCC1}$ & $\mathrm{BCC} 2$ \\
\hline Average Efficiency & 0.524 & 0.573 & 0.642 & 0.698 & 0.767 & 0.801 \\
\hline Standard Deviation & 0.112 & 0.115 & 0.114 & 0.112 & 0.123 & 0.114 \\
\hline \multicolumn{7}{|c|}{ Greater Toronto Area- East } \\
\hline Model/Run & CCR1 & CCR2 & CCR3 & CCR4 & BCC1 & BCC2 \\
\hline Average Efficiency & 0.539 & 0.600 & 0.677 & 0.750 & 0.810 & 0.850 \\
\hline Standard Deviation & 0.095 & 0.135 & 0.139 & 0.139 & 0.144 & 0.139 \\
\hline \multicolumn{7}{|c|}{ Southern Ontario } \\
\hline Model/Run & CCR1 & CCR2 & CCR3 & CCR4 & BCC1 & $\mathrm{BCC} 2$ \\
\hline Average Efficiency & 0.510 & 0.568 & 0.643 & 0.687 & 0.698 & 0.750 \\
\hline Standard Deviation & 0.110 & 0.145 & 0.148 & 0.134 & 0.128 & 0.127 \\
\hline \multicolumn{7}{|c|}{ Prairies (Manitoba and Saskatchewan) } \\
\hline Model/Run & CCR1 & CCR2 & CCR3 & CCR4 & BCC1 & BCC2 \\
\hline Average Efficiency & 0.589 & 0.655 & 0.731 & 0.780 & 0.756 & 0.845 \\
\hline Standard Deviation & 0.158 & 0.164 & 0.162 & 0.159 & 0.155 & 0.147 \\
\hline \multicolumn{7}{|c|}{ Alberta } \\
\hline Model/Run & CCR1 & CCR2 & CCR3 & CCR4 & BCC1 & $\mathrm{BCC} 2$ \\
\hline Average Efficiency & 0.531 & 0.595 & 0.673 & 0.722 & 0.744 & 0.792 \\
\hline Standard Deviation & 0.087 & 0.110 & 0.115 & 0.111 & 0.113 & 0.105 \\
\hline \multicolumn{7}{|c|}{ Vancouver Lower Mainland } \\
\hline Model/Run & CCR1 & CCR2 & CCR3 & CCR4 & $\mathrm{BCC1}$ & $\mathrm{BCC} 2$ \\
\hline Average Efficiency & 0.532 & 0.599 & 0.669 & 0.727 & 0.783 & 0.818 \\
\hline Standard Deviation & 0.102 & 0.134 & 0.138 & 0.127 & 0.105 & 0.104 \\
\hline \multicolumn{7}{|c|}{ Vancouver Island, Central BC, Northern Territories } \\
\hline Model/Run & CCR1 & CCR2 & CCR3 & CCR4 & $\mathrm{BCC1}$ & BCC2 \\
\hline Average Efficiency & 0.552 & 0.614 & 0.688 & 0.735 & 0.774 & 0.814 \\
\hline Standard Deviation & 0.109 & 0.126 & 0.129 & 0.118 & 0.111 & 0.104 \\
\hline
\end{tabular}

Urban/Rural-

\begin{tabular}{|l|c|c|c|c|c|c|}
\hline \hline \multicolumn{7}{|c|}{ Urban } \\
\hline Model/Run & CCR1 & CCR2 & CCR3 & CCR4 & BCC1 & BCC2 \\
\hline Average Efficiency & 0.515 & 0.578 & 0.651 & 0.714 & 0.781 & 0.817 \\
\hline Standard Deviation & 0.088 & 0.104 & 0.114 & 0.116 & 0.114 & 0.111 \\
\hline \multicolumn{7}{|l|}{ Rural } \\
\hline Model/Run & CCR1 & CCR2 & CCR3 & CCR4 & BCC1 & BCC2 \\
\hline Average Efficiency & 0.563 & 0.628 & 0.699 & 0.747 & 0.742 & 0.804 \\
\hline Standard Deviation & 0.128 & 0.150 & 0.147 & 0.141 & 0.133 & 0.131 \\
\hline
\end{tabular}


Funds Managed-

\begin{tabular}{|c|c|c|c|c|c|c|}
\hline \multicolumn{7}{|c|}{$3,313,956.60-80,282,296.80$} \\
\hline Model/Run & CCR1 & CCR2 & CCR3 & CCR4 & BCC1 & $\mathrm{BCC} 2$ \\
\hline Average Efficiency & 0.552 & 0.619 & 0.694 & 0.743 & 0.723 & 0.787 \\
\hline Standard Deviation & 0.137 & 0.155 & 0.154 & 0.148 & 0.136 & 0.137 \\
\hline \multicolumn{7}{|c|}{$80,481,614.72-170,379,805.97$} \\
\hline Model/Run & CCR1 & CCR2 & CCR3 & CCR4 & BCC1 & $\mathrm{BCC} 2$ \\
\hline Average Efficiency & 0.522 & 0.582 & 0.654 & 0.718 & 0.786 & 0.820 \\
\hline Standard Deviation & 0.074 & 0.093 & 0.103 & 0.108 & 0.095 & 0.097 \\
\hline \multicolumn{7}{|c|}{$171,200,464.57-336,017,752.75$} \\
\hline Model/Run & CCR1 & CCR2 & CCR3 & CCR4 & BCC1 & $\mathrm{BCC} 2$ \\
\hline Average Efficiency & 0.522 & 0.584 & 0.642 & 0.709 & 0.847 & 0.868 \\
\hline Standard Deviation & 0.073 & 0.091 & 0.091 & 0.098 & 0.088 & 0.089 \\
\hline \multicolumn{7}{|c|}{$359,066,886.41-1,165,724,792.51$} \\
\hline Model/Run & CCR1 & CCR2 & CCR3 & CCR4 & BCC1 & $\mathrm{BCC} 2$ \\
\hline Average Efficiency & 0.535 & 0.615 & 0.679 & 0.691 & 0.888 & 0.901 \\
\hline Standard Deviation & 0.086 & 0.137 & 0.162 & 0.112 & 0.095 & 0.103 \\
\hline
\end{tabular}

Existing Customer Base-

\begin{tabular}{|c|c|c|c|c|c|c|}
\hline \hline \multicolumn{7}{|c|}{ 342- 4,092 Customers } \\
\hline Model/Run & CCR1 & CCR2 & CCR3 & CCR4 & BCC1 & BCC2 \\
\hline Average Efficiency & 0.572 & 0.644 & 0.718 & 0.763 & 0.720 & 0.792 \\
\hline Standard Deviation & 0.142 & 0.161 & 0.157 & 0.151 & 0.141 & 0.143 \\
\hline \multicolumn{7}{|c|}{$4,117-8,038$ Customers } \\
\hline Model/Run & CCR1 & CCR2 & CCR3 & CCR4 & BCC1 & BCC2 \\
\hline Average Efficiency & 0.519 & 0.580 & 0.656 & 0.720 & 0.763 & 0.804 \\
\hline Standard Deviation & 0.081 & 0.097 & 0.110 & 0.114 & 0.102 & 0.107 \\
\hline \multicolumn{7}{|c|}{$8,186-14,314$ Customers } \\
\hline Model/Run & CCR1 & CCR2 & CCR3 & CCR4 & BCC1 & BCC2 \\
\hline Average Efficiency & 0.505 & 0.563 & 0.629 & 0.696 & 0.827 & 0.841 \\
\hline Standard Deviation & 0.072 & 0.092 & 0.092 & 0.100 & 0.090 & 0.094 \\
\hline Model/Run & $14,524-37,198$ Customers & & \\
\hline Average Efficiency & CCR1 & CCR2 & CCR3 & CCR4 & BCC1 & BCC2 \\
\hline Standard Deviation & 0.510 & 0.565 & 0.624 & 0.681 & 0.878 & 0.895 \\
\hline
\end{tabular}

\section{Returns to Scale-}

\begin{tabular}{|c|c|c|c|c|c|c|}
\hline \hline \multicolumn{7}{|c|}{ All DMUs } \\
\hline Model/Run & CCR1 & CCR2 & CCR3 & CCR4 & BCC1 & BCC2 \\
\hline CRS & $100.0 \%$ & $100.0 \%$ & $100.0 \%$ & $100.0 \%$ & $0.1 \%$ & $0.2 \%$ \\
\hline IRS & $0.0 \%$ & $0.0 \%$ & $0.0 \%$ & $0.0 \%$ & $0.4 \%$ & $5.9 \%$ \\
\hline DRS & $0.0 \%$ & $0.0 \%$ & $0.0 \%$ & $0.0 \%$ & $99.5 \%$ & $93.9 \%$ \\
\hline \multicolumn{7}{|c|}{ Funds-S } \\
\hline Model/Run & CCR1 & CCR2 & CCR3 & CCR4 & BCC1 & BCC2 \\
\hline CRS & $100.0 \%$ & $100.0 \%$ & $100.0 \%$ & $100.0 \%$ & $0.2 \%$ & $0.4 \%$ \\
\hline IRS & $0.0 \%$ & $0.0 \%$ & $0.0 \%$ & $0.0 \%$ & $0.8 \%$ & $12.1 \%$ \\
\hline DRS & $0.0 \%$ & $0.0 \%$ & $0.0 \%$ & $0.0 \%$ & $99.0 \%$ & $87.4 \%$ \\
\hline \multicolumn{7}{|c|}{ Funds-M $\%$} \\
\hline Model/Run & CCR1 & CCR2 & CCR3 & CCR4 & BCC1 & BCC2 \\
\hline CRS & $100.0 \%$ & $100.0 \%$ & $100.0 \%$ & $100.0 \%$ & $0.0 \%$ & $0.0 \%$ \\
\hline IRS & $0.0 \%$ & $0.0 \%$ & $0.0 \%$ & $0.0 \%$ & $0.0 \%$ & $0.3 \%$ \\
\hline DRS & $0.0 \%$ & $0.0 \%$ & $0.0 \%$ & $0.0 \%$ & $100.0 \%$ & $99.7 \%$ \\
\hline \multicolumn{7}{|c|}{ Funds-L } \\
\hline Model/Run & CCR1 & CCR2 & CCR3 & CCR4 & BCC1 & BCC2 \\
\hline CRS & $100.0 \%$ & $100.0 \%$ & $100.0 \%$ & $100.0 \%$ & $0.0 \%$ & $0.0 \%$ \\
\hline IRS & $0.0 \%$ & $0.0 \%$ & $0.0 \%$ & $0.0 \%$ & $0.0 \%$ & $0.0 \%$ \\
\hline DRS & $0.0 \%$ & $0.0 \%$ & $0.0 \%$ & $0.0 \%$ & $100.0 \%$ & $100.0 \%$ \\
\hline \multicolumn{7}{|c|}{ Funds-XL } \\
\hline Model/Run & CCR1 & CCR2 & CCR3 & CCR4 & BCC1 & BCC2 \\
\hline CRS & $100.0 \%$ & $100.0 \%$ & $100.0 \%$ & $100.0 \%$ & $0.0 \%$ & $0.0 \%$ \\
\hline IRS & $0.0 \%$ & $0.0 \%$ & $0.0 \%$ & $0.0 \%$ & $0.0 \%$ & $0.0 \%$ \\
\hline DRS & $0.0 \%$ & $0.0 \%$ & $0.0 \%$ & $0.0 \%$ & $100.0 \%$ & $100.0 \%$ \\
\hline
\end{tabular}




\section{Market Model: Component Model}

\begin{tabular}{|c|c|c|c|c|c|c|}
\hline \multicolumn{7}{|c|}{ Altlantic Canada } \\
\hline Model/Run & CCR1 & CCR2 & CCR3 & BCC1 & $\mathrm{BCC} 2$ & BCC3 \\
\hline Average Efficiency & 0.899 & 0.903 & 0.951 & 0.916 & 0.916 & 0.968 \\
\hline Standard Deviation & 0.111 & 0.112 & 0.081 & 0.107 & 0.107 & 0.064 \\
\hline \multicolumn{7}{|c|}{ Quebec } \\
\hline Model/Run & CCR1 & CCR2 & CCR3 & $\mathrm{BCC} 1$ & $\mathrm{BCC} 2$ & BCC3 \\
\hline Average Efficiency & 0.927 & 0.931 & 0.983 & 0.949 & 0.951 & 0.978 \\
\hline Standard Deviation & 0.112 & 0.111 & 0.064 & 0.102 & 0.102 & 0.071 \\
\hline \multicolumn{7}{|c|}{ Northern Ontario } \\
\hline Model/Run & CCR1 & CCR2 & CCR3 & $\mathrm{BCC} 1$ & $\mathrm{BCC} 2$ & BCC3 \\
\hline Average Efficiency & 0.947 & 0.950 & 0.985 & 0.968 & 0.969 & 0.991 \\
\hline Standard Deviation & 0.080 & 0.079 & 0.043 & 0.067 & 0.067 & 0.038 \\
\hline \multicolumn{7}{|c|}{ Greater Toronto Area- Central } \\
\hline Model/Run & CCR1 & CCR2 & CCR3 & $\mathrm{BCC} 1$ & $\mathrm{BCC} 2$ & BCC3 \\
\hline Average Efficiency & 0.932 & 0.941 & 0.988 & 0.949 & 0.956 & 0.993 \\
\hline Standard Deviation & 0.095 & 0.093 & 0.034 & 0.089 & 0.085 & 0.026 \\
\hline \multicolumn{7}{|c|}{ Greater Toronto Area- West } \\
\hline Model/Run & CCR1 & CCR2 & CCR3 & BCC1 & $\mathrm{BCC} 2$ & BCC3 \\
\hline Average Efficiency & 0.904 & 0.906 & 0.997 & 0.942 & 0.940 & 1.000 \\
\hline Standard Deviation & 0.108 & 0.109 & 0.019 & 0.096 & 0.098 & 0.000 \\
\hline \multicolumn{7}{|c|}{ Greater Toronto Area- East } \\
\hline Model/Run & CCR1 & CCR2 & CCR3 & BCC1 & $\mathrm{BCC} 2$ & BCC3 \\
\hline Average Efficiency & 0.927 & 0.940 & 0.980 & 0.967 & 0.966 & 0.986 \\
\hline Standard Deviation & 0.089 & 0.097 & 0.068 & 0.444 & 0.121 & 0.133 \\
\hline \multicolumn{7}{|c|}{ Southern Ontario } \\
\hline Model/Run & CCR1 & CCR2 & CCR3 & BCC1 & $\mathrm{BCC} 2$ & BCC3 \\
\hline Average Efficiency & 0.928 & 0.942 & 0.991 & 0.950 & 0.959 & 0.996 \\
\hline Standard Deviation & 0.087 & 0.085 & 0.037 & 0.084 & 0.077 & 0.022 \\
\hline \multicolumn{7}{|c|}{ Prairies (Manitoba and Saskatchewan) } \\
\hline Model/Run & CCR1 & CCR2 & CCR3 & BCC1 & $\mathrm{BCC} 2$ & BCC3 \\
\hline Average Efficiency & 0.930 & 0.929 & 0.981 & 0.962 & 0.961 & 0.997 \\
\hline Standard Deviation & 0.103 & 0.103 & 0.044 & 0.084 & 0.085 & 0.016 \\
\hline \multicolumn{7}{|c|}{ Alberta } \\
\hline Model/Run & CCR1 & CCR2 & CCR3 & BCC1 & $\mathrm{BCC} 2$ & BCC3 \\
\hline Average Efficiency & 0.911 & 0.922 & 0.982 & 0.940 & 0.943 & 0.989 \\
\hline Standard Deviation & 0.108 & 0.108 & 0.052 & 0.100 & 0.097 & 0.032 \\
\hline \multicolumn{7}{|c|}{ Vancouver Lower Mainland } \\
\hline Model/Run & CCR1 & CCR2 & CCR3 & $\mathrm{BCC} 1$ & $\mathrm{BCC} 2$ & BCC3 \\
\hline Average Efficiency & 0.905 & 0.900 & 0.983 & 0.942 & 0.938 & 0.984 \\
\hline Standard Deviation & 0.099 & 0.093 & 0.052 & 0.090 & 0.091 & 0.050 \\
\hline \multicolumn{7}{|c|}{ Vancouver Island, Central BC, Northern Territories } \\
\hline Model/Run & CCR1 & CCR2 & CCR3 & BCC1 & $\mathrm{BCC} 2$ & BCC3 \\
\hline Average Efficiency & 0.908 & 0.911 & 0.985 & 0.943 & 0.944 & 0.989 \\
\hline Standard Deviation & 0.100 & 0.098 & 0.051 & 0.091 & 0.090 & 0.041 \\
\hline
\end{tabular}

Urban/Rural-

\begin{tabular}{|l|c|c|c|c|c|c|}
\hline \hline \multicolumn{7}{|c|}{ Urban } \\
\hline Model/Run & CCR1 & CCR2 & CCR3 & BCC1 & BCC2 & BCC3 \\
\hline Average Efficiency & 0.908 & 0.917 & 0.982 & 0.942 & 0.943 & 0.988 \\
\hline Standard Deviation & 0.103 & 0.103 & 0.051 & 0.096 & 0.096 & 0.044 \\
\hline \multicolumn{7}{|c|}{ Rural } \\
\hline Model/Run & CCR1 & CCR2 & CCR3 & BCC1 & BCC2 & BCC3 \\
\hline Average Efficiency & 0.937 & 0.940 & 0.984 & 0.958 & 0.961 & 0.990 \\
\hline Standard Deviation & 0.094 & 0.093 & 0.048 & 0.083 & 0.081 & 0.039 \\
\hline
\end{tabular}


Funds Managed-

\begin{tabular}{|c|c|c|c|c|c|c|}
\hline \multicolumn{7}{|c|}{$3,313,956.60-80,282,296.80$} \\
\hline Model/Run & CCR1 & CCR2 & CCR3 & BCC1 & $\mathrm{BCC} 2$ & BCC3 \\
\hline Average Efficiency & 0.921 & 0.926 & 0.982 & 0.945 & 0.947 & 0.986 \\
\hline Standard Deviation & 0.108 & 0.107 & 0.052 & 0.099 & 0.097 & 0.046 \\
\hline \multicolumn{7}{|c|}{$80,481,614.72-170,379,805.97$} \\
\hline Model/Run & CCR1 & CCR2 & CCR3 & BCC1 & $\mathrm{BCC} 2$ & BCC3 \\
\hline Average Efficiency & 0.910 & 0.919 & 0.982 & 0.943 & 0.946 & 0.989 \\
\hline Standard Deviation & 0.096 & 0.095 & 0.051 & 0.088 & 0.087 & 0.041 \\
\hline \multicolumn{7}{|c|}{$171,200,464.57-336,017,752.75$} \\
\hline Model/Run & CCR1 & CCR2 & CCR3 & $\mathrm{BCC1}$ & BCC2 & $\mathrm{BCC} 3$ \\
\hline Average Efficiency & 0.956 & 0.962 & 0.996 & 0.984 & 0.983 & 1.000 \\
\hline Standard Deviation & 0.069 & 0.068 & 0.020 & 0.046 & 0.048 & 0.000 \\
\hline \multicolumn{7}{|c|}{$359,066,886.41-1,165,724,792.51$} \\
\hline Model/Run & CCR1 & CCR2 & CCR3 & $\mathrm{BCC1}$ & $\mathrm{BCC} 2$ & $\mathrm{BCC} 3$ \\
\hline Average Efficiency & 0.959 & 0.988 & 1.000 & 1.000 & 1.000 & 1.000 \\
\hline Standard Deviation & 0.064 & 0.048 & N/A & 0.000 & 0.000 & N/A \\
\hline
\end{tabular}

Existing Customer Base-

\begin{tabular}{|c|c|c|c|c|c|c|}
\hline \hline \multicolumn{7}{|c|}{ 342- 4,092 Customers } \\
\hline Model/Run & CCR1 & CCR2 & CCR3 & BCC1 & BCC2 & BCC3 \\
\hline Average Efficiency & 0.942 & 0.946 & 0.995 & 0.964 & 0.967 & 0.999 \\
\hline Standard Deviation & 0.095 & 0.093 & 0.029 & 0.082 & 0.080 & 0.010 \\
\hline \multicolumn{7}{|c|}{$4,117-8,038$ Customers } \\
\hline Model/Run & CCR1 & CCR2 & CCR3 & BCC1 & BCC2 & BCC3 \\
\hline Average Efficiency & 0.894 & 0.903 & 0.975 & 0.924 & 0.928 & 0.981 \\
\hline Standard Deviation & 0.106 & 0.106 & 0.061 & 0.102 & 0.101 & 0.053 \\
\hline \multicolumn{7}{|c|}{$8,186-14,314$ Customers } \\
\hline Model/Run & CCR1 & CCR2 & CCR3 & BCC1 & BCC2 & BCC3 \\
\hline Average Efficiency & 0.924 & 0.933 & 0.986 & 0.961 & 0.960 & 0.994 \\
\hline Standard Deviation & 0.092 & 0.091 & 0.040 & 0.078 & 0.078 & 0.030 \\
\hline Model/Run & $14,524-37,198$ Customers & & \\
\hline Average Efficiency & CCR1 & CCR2 & CCR3 & BCC1 & BCC2 & BCC3 \\
\hline Standard Deviation & 0.964 & 0.977 & 1.000 & 0.996 & 0.996 & 1.000 \\
\hline
\end{tabular}

\section{Returns to Scale-}

\begin{tabular}{|c|c|c|c|c|c|c|}
\hline \hline \multicolumn{7}{|c|}{ All DMUs } \\
\hline Model/Run & CCR1 & CCR2 & CCR3 & BCC1 & BCC2 & BCC3 \\
\hline CRS & $100.0 \%$ & $100.0 \%$ & $100.0 \%$ & $2.3 \%$ & $6.1 \%$ & $13.3 \%$ \\
\hline IRS & $0.0 \%$ & $0.0 \%$ & $0.0 \%$ & $21.5 \%$ & $34.6 \%$ & $29.5 \%$ \\
\hline DRS & $0.0 \%$ & $0.0 \%$ & $0.0 \%$ & $76.2 \%$ & $59.3 \%$ & $57.3 \%$ \\
\hline \multicolumn{7}{|c|}{ Funds-S } \\
\hline Model/Run & CCR1 & CCR2 & CCR3 & BCC1 & BCC2 & BCC3 \\
\hline CRS & $100.0 \%$ & $100.0 \%$ & $100.0 \%$ & $3.4 \%$ & $7.2 \%$ & $14.3 \%$ \\
\hline IRS & $0.0 \%$ & $0.0 \%$ & $0.0 \%$ & $29.6 \%$ & $43.3 \%$ & $43.4 \%$ \\
\hline DRS & $0.0 \%$ & $0.0 \%$ & $0.0 \%$ & $67.0 \%$ & $49.5 \%$ & $42.3 \%$ \\
\hline \multicolumn{7}{|c|}{ Funds-M } \\
\hline Model/Run & CCR1 & CCR2 & CCR3 & BCC1 & BCC2 & BCC3 \\
\hline CRS & $100.0 \%$ & $100.0 \%$ & $100.0 \%$ & $1.3 \%$ & $2.8 \%$ & $13.4 \%$ \\
\hline IRS & $0.0 \%$ & $0.0 \%$ & $0.0 \%$ & $15.5 \%$ & $29.3 \%$ & $19.4 \%$ \\
\hline DRS & $0.0 \%$ & $0.0 \%$ & $0.0 \%$ & $83.2 \%$ & $67.9 \%$ & $67.2 \%$ \\
\hline \multicolumn{7}{|c|}{ Funds-L } \\
\hline Model/Run & CCR1 & CCR2 & CCR3 & BCC1 & BCC2 & BCC3 \\
\hline CRS & $100.0 \%$ & $100.0 \%$ & $100.0 \%$ & $0.9 \%$ & $13.0 \%$ & $6.5 \%$ \\
\hline IRS & $0.0 \%$ & $0.0 \%$ & $0.0 \%$ & $7.8 \%$ & $17.4 \%$ & $9.7 \%$ \\
\hline DRS & $0.0 \%$ & $0.0 \%$ & $0.0 \%$ & $91.3 \%$ & $69.6 \%$ & $83.9 \%$ \\
\hline \multicolumn{7}{|c|}{ Funds-XL } \\
\hline Model/Run & CCR1 & CCR2 & CCR3 & BCC1 & BCC2 & BCC3 \\
\hline CRS & $100.0 \%$ & $100.0 \%$ & $100.0 \%$ & $0.0 \%$ & $0.0 \%$ & $0.0 \%$ \\
\hline IRS & $0.0 \%$ & $0.0 \%$ & $0.0 \%$ & $0.0 \%$ & $20.0 \%$ & $0.0 \%$ \\
\hline DRS & $0.0 \%$ & $0.0 \%$ & $0.0 \%$ & $100.0 \%$ & $80.0 \%$ & $100.0 \%$ \\
\hline
\end{tabular}




\section{Market Model: Aggregate Model}

\begin{tabular}{|c|c|c|c|c|c|c|}
\hline \multicolumn{7}{|c|}{ Altlantic Canada } \\
\hline Model/Run & CCR1 & CCR2 & CCR3 & BCC1 & $\mathrm{BCC} 2$ & BCC3 \\
\hline Average Efficiency & 0.849 & 0.855 & 0.906 & 0.881 & 0.881 & 0.920 \\
\hline Standard Deviation & 0.118 & 0.121 & 0.094 & 0.119 & 0.119 & 0.087 \\
\hline \multicolumn{7}{|c|}{ Quebec } \\
\hline Model/Run & CCR1 & CCR2 & CCR3 & $\mathrm{BCC} 1$ & $\mathrm{BCC} 2$ & BCC3 \\
\hline Average Efficiency & 0.866 & 0.877 & 0.942 & 0.910 & 0.910 & 0.937 \\
\hline Standard Deviation & 0.127 & 0.127 & 0.097 & 0.122 & 0.123 & 0.111 \\
\hline \multicolumn{7}{|c|}{ Northern Ontario } \\
\hline Model/Run & CCR1 & CCR2 & CCR3 & $\mathrm{BCC} 1$ & $\mathrm{BCC} 2$ & BCC3 \\
\hline Average Efficiency & 0.903 & 0.906 & 0.961 & 0.934 & 0.937 & 0.965 \\
\hline Standard Deviation & 0.097 & 0.098 & 0.067 & 0.092 & 0.092 & 0.068 \\
\hline \multicolumn{7}{|c|}{ Greater Toronto Area- Central } \\
\hline Model/Run & CCR1 & CCR2 & CCR3 & $\mathrm{BCC} 1$ & $\mathrm{BCC} 2$ & BCC3 \\
\hline Average Efficiency & 0.883 & 0.903 & 0.950 & 0.920 & 0.929 & 0.988 \\
\hline Standard Deviation & 0.100 & 0.100 & 0.084 & 0.100 & 0.094 & 0.030 \\
\hline \multicolumn{7}{|c|}{ Greater Toronto Area- West } \\
\hline Model/Run & CCR1 & CCR2 & CCR3 & BCC1 & $\mathrm{BCC} 2$ & BCC3 \\
\hline Average Efficiency & 0.838 & 0.860 & 0.948 & 0.897 & 0.899 & 0.989 \\
\hline Standard Deviation & 0.103 & 0.111 & 0.079 & 0.111 & 0.114 & 0.036 \\
\hline \multicolumn{7}{|c|}{ Greater Toronto Area- East } \\
\hline Model/Run & CCR1 & CCR2 & CCR3 & BCC1 & $\mathrm{BCC} 2$ & BCC3 \\
\hline Average Efficiency & 0.879 & 0.911 & 0.945 & 0.924 & 0.930 & 1.000 \\
\hline Standard Deviation & 0.099 & 0.110 & 0.087 & 0.319 & 0.133 & 0.171 \\
\hline \multicolumn{7}{|c|}{ Southern Ontario } \\
\hline Model/Run & CCR1 & CCR2 & CCR3 & BCC1 & $\mathrm{BCC} 2$ & BCC3 \\
\hline Average Efficiency & 0.896 & 0.907 & 0.956 & 0.916 & 0.932 & 0.924 \\
\hline Standard Deviation & 0.100 & 0.100 & 0.073 & 0.102 & 0.097 & 0.097 \\
\hline \multicolumn{7}{|c|}{ Prairies (Manitoba and Saskatchewan) } \\
\hline Model/Run & CCR1 & CCR2 & CCR3 & BCC1 & $\mathrm{BCC} 2$ & BCC3 \\
\hline Average Efficiency & 0.882 & 0.885 & 0.956 & 0.942 & 0.941 & 0.972 \\
\hline Standard Deviation & 0.115 & 0.114 & 0.077 & 0.100 & 0.101 & 0.061 \\
\hline \multicolumn{7}{|c|}{ Alberta } \\
\hline Model/Run & CCR1 & CCR2 & CCR3 & BCC1 & $\mathrm{BCC} 2$ & BCC3 \\
\hline Average Efficiency & 0.851 & 0.874 & 0.958 & 0.894 & 0.904 & 0.967 \\
\hline Standard Deviation & 0.116 & 0.122 & 0.079 & 0.120 & 0.117 & 0.074 \\
\hline \multicolumn{7}{|c|}{ Vancouver Lower Mainland } \\
\hline Model/Run & CCR1 & CCR2 & CCR3 & $\mathrm{BCC} 1$ & $\mathrm{BCC} 2$ & BCC3 \\
\hline Average Efficiency & 0.851 & 0.865 & 0.941 & 0.892 & 0.896 & 0.964 \\
\hline Standard Deviation & 0.102 & 0.106 & 0.079 & 0.106 & 0.106 & 0.067 \\
\hline \multicolumn{7}{|c|}{ Vancouver Island, Central BC, Northern Territories } \\
\hline Model/Run & CCR1 & CCR2 & CCR3 & BCC1 & $\mathrm{BCC} 2$ & BCC3 \\
\hline Average Efficiency & 0.849 & 0.858 & 0.951 & 0.902 & 0.907 & 0.973 \\
\hline Standard Deviation & 0.099 & 0.102 & 0.081 & 0.106 & 0.106 & 0.063 \\
\hline
\end{tabular}

Urban/Rural-

\begin{tabular}{|l|c|c|c|c|c|c|}
\hline \hline \multicolumn{7}{|c|}{ Urban } \\
\hline Model/Run & CCR1 & CCR2 & CCR3 & BCC1 & BCC2 & BCC3 \\
\hline Average Efficiency & 0.851 & 0.871 & 0.941 & 0.900 & 0.904 & 0.945 \\
\hline Standard Deviation & 0.108 & 0.113 & 0.085 & 0.111 & 0.111 & 0.088 \\
\hline \multicolumn{7}{|c|}{ Rural } \\
\hline Model/Run & CCR1 & CCR2 & CCR3 & BCC1 & BCC2 & BCC3 \\
\hline Average Efficiency & 0.893 & 0.898 & 0.958 & 0.926 & 0.931 & 0.966 \\
\hline Standard Deviation & 0.108 & 0.108 & 0.074 & 0.104 & 0.102 & 0.068 \\
\hline
\end{tabular}


Funds Managed-

\begin{tabular}{|c|c|c|c|c|c|c|}
\hline \multicolumn{7}{|c|}{$3,313,956.60-80,282,296.80$} \\
\hline Model/Run & CCR1 & CCR2 & CCR3 & $\mathrm{BCC1}$ & $\mathrm{BCC} 2$ & $\mathrm{BCC} 3$ \\
\hline Average Efficiency & 0.874 & 0.889 & 0.945 & 0.912 & 0.919 & 0.958 \\
\hline Standard Deviation & 0.119 & 0.121 & 0.089 & 0.117 & 0.116 & 0.080 \\
\hline \multicolumn{7}{|c|}{$80,481,614.72-170,379,805.97$} \\
\hline Model/Run & CCR1 & CCR2 & CCR3 & $\mathrm{BCC1}$ & $\mathrm{BCC} 2$ & $\mathrm{BCC} 3$ \\
\hline Average Efficiency & 0.855 & 0.865 & 0.944 & 0.895 & 0.900 & 0.946 \\
\hline Standard Deviation & 0.099 & 0.102 & 0.077 & 0.100 & 0.101 & 0.082 \\
\hline \multicolumn{7}{|c|}{$171,200,464.57-336,017,752.75$} \\
\hline Model/Run & CCR1 & CCR2 & CCR3 & BCC1 & $\mathrm{BCC} 2$ & $\mathrm{BCC} 3$ \\
\hline Average Efficiency & 0.901 & 0.914 & 0.982 & 0.958 & 0.958 & 0.994 \\
\hline Standard Deviation & 0.088 & 0.089 & 0.045 & 0.077 & 0.076 & 0.020 \\
\hline \multicolumn{7}{|c|}{$359,066,886.41-1,165,724,792.51$} \\
\hline Model/Run & CCR1 & CCR2 & CCR3 & $\mathrm{BCC1}$ & $\mathrm{BCC} 2$ & BCC3 \\
\hline Average Efficiency & 0.944 & 0.958 & 1.000 & 0.993 & 0.993 & 1.000 \\
\hline Standard Deviation & 0.079 & 0.077 & 0.000 & 0.028 & 0.028 & 0.000 \\
\hline
\end{tabular}

Existing Customer Base-

\begin{tabular}{|c|c|c|c|c|c|c|}
\hline \multicolumn{7}{|c|}{ 342- 4,092 Customers } \\
\hline Model/Run & CCR1 & CCR2 & CCR3 & BCC1 & BCC2 & BCC3 \\
\hline Average Efficiency & 0.893 & 0.909 & 0.967 & 0.933 & 0.939 & 0.978 \\
\hline Standard Deviation & 0.114 & 0.113 & 0.069 & 0.106 & 0.104 & 0.056 \\
\hline \multicolumn{7}{|c|}{$4,117-8,038$ Customers } \\
\hline Model/Run & CCR1 & CCR2 & CCR3 & BCC1 & $\mathrm{BCC} 2$ & $\mathrm{BCC} 3$ \\
\hline Average Efficiency & 0.843 & 0.855 & 0.930 & 0.879 & 0.885 & 0.937 \\
\hline Standard Deviation & 0.106 & 0.110 & 0.090 & 0.110 & 0.112 & 0.093 \\
\hline \multicolumn{7}{|c|}{ 8,186-14,314 Customers } \\
\hline Model/Run & CCR1 & CCR2 & CCR3 & BCC1 & $\mathrm{BCC} 2$ & $\mathrm{BCC} 3$ \\
\hline Average Efficiency & 0.865 & 0.877 & 0.956 & 0.918 & 0.923 & 0.957 \\
\hline Standard Deviation & 0.096 & 0.100 & 0.070 & 0.097 & 0.097 & 0.071 \\
\hline \multicolumn{7}{|c|}{ 14,524-37,198 Customers } \\
\hline Model/Run & CCR1 & CCR2 & CCR3 & BCC1 & BCC2 & BCC3 \\
\hline Average Efficiency & 0.927 & 0.945 & 0.992 & 0.990 & 0.990 & 1.000 \\
\hline Standard Deviation & 0.084 & 0.082 & 0.031 & 0.040 & 0.040 & 0.000 \\
\hline
\end{tabular}

\section{Returns to Scale-}

\begin{tabular}{|c|c|c|c|c|c|c|}
\hline \hline \multicolumn{7}{|c|}{ All DMUs } \\
\hline Model/Run & CCR1 & CCR2 & CCR3 & BCC1 & BCC2 & BCC3 \\
\hline CRS & $100.0 \%$ & $100.0 \%$ & $100.0 \%$ & $1.7 \%$ & $4.6 \%$ & $8.9 \%$ \\
\hline IRS & $0.0 \%$ & $0.0 \%$ & $0.0 \%$ & $18.9 \%$ & $28.9 \%$ & $29.2 \%$ \\
\hline DRS & $0.0 \%$ & $0.0 \%$ & $0.0 \%$ & $79.3 \%$ & $66.5 \%$ & $62.0 \%$ \\
\hline \multicolumn{7}{|c|}{ Funds-S } \\
\hline Model/Run & CCR1 & CCR2 & CCR3 & BCC1 & BCC2 & BCC3 \\
\hline CRS & $100.0 \%$ & $100.0 \%$ & $100.0 \%$ & $2.9 \%$ & $5.5 \%$ & $9.7 \%$ \\
\hline IRS & $0.0 \%$ & $0.0 \%$ & $0.0 \%$ & $26.7 \%$ & $37.4 \%$ & $40.0 \%$ \\
\hline DRS & $0.0 \%$ & $0.0 \%$ & $0.0 \%$ & $70.4 \%$ & $57.1 \%$ & $50.3 \%$ \\
\hline \multicolumn{7}{|c|}{ Funds-M } \\
\hline Model/Run & CCR1 & CCR2 & CCR3 & BCC1 & BCC2 & BCC3 \\
\hline CRS & $100.0 \%$ & $100.0 \%$ & $100.0 \%$ & $0.8 \%$ & $2.6 \%$ & $7.7 \%$ \\
\hline IRS & $0.0 \%$ & $0.0 \%$ & $0.0 \%$ & $12.4 \%$ & $21.8 \%$ & $23.7 \%$ \\
\hline DRS & $0.0 \%$ & $0.0 \%$ & $0.0 \%$ & $86.8 \%$ & $75.6 \%$ & $68.6 \%$ \\
\hline \multicolumn{7}{|c|}{ Funds-L } \\
\hline Model/Run & CCR1 & CCR2 & CCR3 & BCC1 & BCC2 & BCC3 \\
\hline CRS & $100.0 \%$ & $100.0 \%$ & $100.0 \%$ & $0.0 \%$ & $7.0 \%$ & $11.1 \%$ \\
\hline IRS & $0.0 \%$ & $0.0 \%$ & $0.0 \%$ & $7.8 \%$ & $19.1 \%$ & $6.3 \%$ \\
\hline DRS & $0.0 \%$ & $0.0 \%$ & $0.0 \%$ & $92.2 \%$ & $73.9 \%$ & $82.5 \%$ \\
\hline \multicolumn{7}{|c|}{ Funds-XL } \\
\hline Model/Run & CCR1 & CCR2 & CCR3 & BCC1 & BCC2 & BCC3 \\
\hline CRS & $100.0 \%$ & $100.0 \%$ & $100.0 \%$ & $0.0 \%$ & $6.7 \%$ & $0.0 \%$ \\
\hline IRS & $0.0 \%$ & $0.0 \%$ & $0.0 \%$ & $0.0 \%$ & $13.3 \%$ & $0.0 \%$ \\
\hline DRS & $0.0 \%$ & $0.0 \%$ & $0.0 \%$ & $100.0 \%$ & $80.0 \%$ & $100.0 \%$ \\
\hline
\end{tabular}




\section{Delta Model: Difference Model by Stripping}

Market-

\begin{tabular}{|c|c|c|c|c|c|c|}
\hline \multicolumn{7}{|c|}{ Altlantic Canada } \\
\hline Model/Run & CCR1 & CCR2 & CCR3 & CCR4 & SBM1 & SBM2 \\
\hline Average Efficiency & 0.340 & 0.486 & 0.522 & 0.569 & 0.213 & 0.430 \\
\hline Standard Deviation & 0.121 & 0.149 & 0.143 & 0.133 & 0.074 & 0.132 \\
\hline \multicolumn{7}{|c|}{ Quebec } \\
\hline Model/Run & CCR1 & CCR2 & CCR3 & CCR4 & SBM1 & SBM2 \\
\hline Average Efficiency & 0.370 & 0.518 & 0.563 & 0.623 & 0.233 & 0.518 \\
\hline Standard Deviation & 0.096 & 0.116 & 0.126 & 0.128 & 0.055 & 0.140 \\
\hline \multicolumn{7}{|c|}{ Northern Ontario } \\
\hline Model/Run & CCR1 & CCR2 & CCR3 & CCR4 & SBM1 & SBM2 \\
\hline Average Efficiency & 0.327 & 0.478 & 0.516 & 0.584 & 0.216 & 0.456 \\
\hline Standard Deviation & 0.073 & 0.106 & 0.101 & 0.118 & 0.057 & 0.122 \\
\hline \multicolumn{7}{|c|}{ Greater Toronto Area- Central } \\
\hline Model/Run & CCR1 & CCR2 & CCR3 & CCR4 & SBM1 & SBM2 \\
\hline Average Efficiency & 0.414 & 0.550 & 0.592 & 0.659 & 0.269 & 0.556 \\
\hline Standard Deviation & 0.149 & 0.141 & 0.136 & 0.147 & 0.126 & 0.152 \\
\hline \multicolumn{7}{|c|}{ Greater Toronto Area- West } \\
\hline Model/Run & CCR1 & CCR2 & CCR3 & CCR4 & SBM1 & SBM2 \\
\hline Average Efficiency & 0.371 & 0.514 & 0.559 & 0.614 & 0.229 & 0.506 \\
\hline Standard Deviation & 0.112 & 0.132 & 0.142 & 0.137 & 0.058 & 0.153 \\
\hline \multicolumn{7}{|c|}{ Greater Toronto Area- East } \\
\hline Model/Run & CCR1 & CCR2 & CCR3 & CCR4 & SBM1 & SBM2 \\
\hline Average Efficiency & 0.432 & 0.565 & 0.607 & 0.666 & 0.275 & 0.565 \\
\hline Standard Deviation & 0.166 & 0.124 & 0.131 & 0.140 & 0.083 & 0.148 \\
\hline \multicolumn{7}{|c|}{ Southern Ontario } \\
\hline Model/Run & CCR1 & CCR2 & CCR3 & CCR4 & SBM1 & SBM2 \\
\hline Average Efficiency & 0.314 & 0.441 & 0.482 & 0.536 & 0.196 & 0.428 \\
\hline Standard Deviation & 0.058 & 0.067 & 0.075 & 0.081 & 0.039 & 0.101 \\
\hline \multicolumn{7}{|c|}{ Prairies (Manitoba and Saskatchewan) } \\
\hline Model/Run & CCR1 & CCR2 & CCR3 & CCR4 & SBM1 & SBM2 \\
\hline Average Efficiency & 0.342 & 0.473 & 0.513 & 0.569 & 0.220 & 0.463 \\
\hline Standard Deviation & 0.117 & 0.123 & 0.123 & 0.133 & 0.098 & 0.150 \\
\hline \multicolumn{7}{|c|}{ Alberta } \\
\hline Model/Run & CCR1 & CCR2 & CCR3 & CCR4 & SBM1 & SBM2 \\
\hline Average Efficiency & 0.359 & 0.504 & 0.545 & 0.604 & 0.221 & 0.487 \\
\hline Standard Deviation & 0.098 & 0.129 & 0.130 & 0.142 & 0.063 & 0.154 \\
\hline \multicolumn{7}{|c|}{ Vancouver Lower Mainland } \\
\hline Model/Run & CCR1 & CCR2 & CCR3 & CCR4 & SBM1 & SBM2 \\
\hline Average Efficiency & 0.372 & 0.563 & 0.602 & 0.667 & 0.257 & 0.532 \\
\hline Standard Deviation & 0.122 & 0.148 & 0.146 & 0.150 & 0.103 & 0.152 \\
\hline \multicolumn{7}{|c|}{ Vancouver Island, Central BC, Northern Territories } \\
\hline Model/Run & CCR1 & CCR2 & CCR3 & CCR4 & SBM1 & SBM2 \\
\hline Average Efficiency & 0.330 & 0.478 & 0.521 & 0.585 & 0.210 & 0.458 \\
\hline Standard Deviation & 0.064 & 0.089 & 0.098 & 0.114 & 0.050 & 0.128 \\
\hline
\end{tabular}

Urban/Rural-

\begin{tabular}{|l|c|c|c|c|c|c|}
\hline \hline \multicolumn{7}{|c|}{ Urban } \\
\hline Model/Run & CCR1 & CCR2 & CCR3 & CCR4 & SBM1 & SBM2 \\
\hline Average Efficiency & 0.399 & 0.549 & 0.590 & 0.653 & 0.255 & 0.537 \\
\hline Standard Deviation & 0.136 & 0.145 & 0.144 & 0.147 & 0.101 & 0.162 \\
\hline \multicolumn{7}{|c|}{ Rural } \\
\hline Model/Run & CCR1 & CCR2 & CCR3 & CCR4 & SBM1 & SBM2 \\
\hline Average Efficiency & 0.319 & 0.454 & 0.494 & 0.549 & 0.200 & 0.435 \\
\hline Standard Deviation & 0.065 & 0.085 & 0.088 & 0.097 & 0.044 & 0.107 \\
\hline
\end{tabular}


Funds Managed-

\begin{tabular}{|c|c|c|c|c|c|c|}
\hline \multicolumn{7}{|c|}{$3,313,956.60-80,282,296.80$} \\
\hline Model/Run & CCR1 & CCR2 & CCR3 & CCR4 & SBM1 & SBM2 \\
\hline Average Efficiency & 0.324 & 0.456 & 0.494 & 0.547 & 0.201 & 0.437 \\
\hline Standard Deviation & 0.083 & 0.095 & 0.094 & 0.096 & 0.042 & 0.100 \\
\hline \multicolumn{7}{|c|}{$80,481,614.72-170,379,805.97$} \\
\hline Model/Run & CCR1 & CCR2 & CCR3 & CCR4 & SBM1 & SBM2 \\
\hline Average Efficiency & 0.372 & 0.524 & 0.567 & 0.633 & 0.237 & 0.516 \\
\hline Standard Deviation & 0.102 & 0.123 & 0.125 & 0.129 & 0.063 & 0.152 \\
\hline \multicolumn{7}{|c|}{$171,200,464.57-336,017,752.75$} \\
\hline Model/Run & CCR1 & CCR2 & CCR3 & CCR4 & SBM1 & SBM2 \\
\hline Average Efficiency & 0.477 & 0.636 & 0.688 & 0.762 & 0.316 & 0.629 \\
\hline Standard Deviation & 0.165 & 0.147 & 0.148 & 0.145 & 0.156 & 0.184 \\
\hline \multicolumn{7}{|c|}{$359,066,886.41-1,165,724,792.51$} \\
\hline Model/Run & CCR1 & CCR2 & CCR3 & CCR4 & SBM1 & SBM2 \\
\hline Average Efficiency & 0.522 & 0.710 & 0.710 & 0.801 & 0.356 & 0.651 \\
\hline Standard Deviation & 0.201 & 0.216 & 0.191 & 0.210 & 0.214 & 0.276 \\
\hline
\end{tabular}

Existing Customer Base-

\begin{tabular}{|c|c|c|c|c|c|c|}
\hline \hline \multicolumn{7}{|c|}{$342-4,092$ Customers } \\
\hline \hline Model/Run & CCR1 & CCR2 & CCR3 & CCR4 & SBM1 & SBM2 \\
\hline Average Efficiency & 0.313 & 0.444 & 0.482 & 0.533 & 0.194 & 0.420 \\
\hline Standard Deviation & 0.072 & 0.086 & 0.087 & 0.088 & 0.036 & 0.088 \\
\hline \multicolumn{7}{|c|}{$4,117-8,038$ Customers } \\
\hline Model/Run & CCR1 & CCR2 & CCR3 & CCR4 & SBM1 & SBM2 \\
\hline Average Efficiency & 0.352 & 0.498 & 0.541 & 0.605 & 0.225 & 0.494 \\
\hline Standard Deviation & 0.086 & 0.102 & 0.106 & 0.113 & 0.049 & 0.125 \\
\hline \multicolumn{7}{|c|}{$8,186-14,314$ Customers } \\
\hline Model/Run & CCR1 & CCR2 & CCR3 & CCR4 & SBM1 & SBM2 \\
\hline Average Efficiency & 0.438 & 0.605 & 0.650 & 0.722 & 0.280 & 0.596 \\
\hline Standard Deviation & 0.128 & 0.150 & 0.143 & 0.142 & 0.097 & 0.180 \\
\hline Model/Run & $14,524-37,198$ Customers & & \\
\hline Average Efficiency & CCR1 & CCR2 & CCR3 & CCR4 & SBM1 & SBM2 \\
\hline Standard Deviation & 0.555 & 0.697 & 0.744 & 0.819 & 0.375 & 0.673 \\
\hline
\end{tabular}

\section{Returns to Scale-}

\begin{tabular}{|c|c|c|c|c|c|c|}
\hline \hline \multicolumn{7}{|c|}{ All DMUs } \\
\hline Model/Run & CCR1 & CCR2 & CCR3 & CCR4 & SBM1 & SBM2 \\
\hline CRS & $100.0 \%$ & $100.0 \%$ & $100.0 \%$ & $100.0 \%$ & $100.0 \%$ & $100.0 \%$ \\
\hline IRS & $0.0 \%$ & $0.0 \%$ & $0.0 \%$ & $0.0 \%$ & $0.0 \%$ & $0.0 \%$ \\
\hline DRS & $0.0 \%$ & $0.0 \%$ & $0.0 \%$ & $0.0 \%$ & $0.0 \%$ & $0.0 \%$ \\
\hline \multicolumn{7}{|c|}{ Funds-S } \\
\hline Model/Run & CCR1 & CCR2 & CCR3 & CCR4 & SBM1 & SBM2 \\
\hline CRS & $100.0 \%$ & $100.0 \%$ & $100.0 \%$ & $100.0 \%$ & $100.0 \%$ & $100.0 \%$ \\
\hline IRS & $0.0 \%$ & $0.0 \%$ & $0.0 \%$ & $0.0 \%$ & $0.0 \%$ & $0.0 \%$ \\
\hline DRS & $0.0 \%$ & $0.0 \%$ & $0.0 \%$ & $0.0 \%$ & $0.0 \%$ & $0.0 \%$ \\
\hline \multicolumn{7}{|c|}{ Funds-M } \\
\hline Model/Run & CCR1 & CCR2 & CCR3 & CCR4 & SBM1 & SBM2 \\
\hline CRS & $100.0 \%$ & $100.0 \%$ & $100.0 \%$ & $100.0 \%$ & $100.0 \%$ & $100.0 \%$ \\
\hline IRS & $0.0 \%$ & $0.0 \%$ & $0.0 \%$ & $0.0 \%$ & $0.0 \%$ & $0.0 \%$ \\
\hline DRS & $0.0 \%$ & $0.0 \%$ & $0.0 \%$ & $0.0 \%$ & $0.0 \%$ & $0.0 \%$ \\
\hline \multicolumn{7}{|c|}{ Funds-L } \\
\hline Model/Run & CCR1 & CCR2 & CCR3 & CCR4 & SBM1 & SBM2 \\
\hline CRS & $100.0 \%$ & $100.0 \%$ & $100.0 \%$ & $100.0 \%$ & $100.0 \%$ & $100.0 \%$ \\
\hline IRS & $0.0 \%$ & $0.0 \%$ & $0.0 \%$ & $0.0 \%$ & $0.0 \%$ & $0.0 \%$ \\
\hline DRS & $0.0 \%$ & $0.0 \%$ & $0.0 \%$ & $0.0 \%$ & $0.0 \%$ & $0.0 \%$ \\
\hline \multicolumn{7}{|c|}{ Funds-XL } \\
\hline Model/Run & CCR1 & CCR2 & CCR3 & CCR4 & SBM1 & SBM2 \\
\hline CRS & $100.0 \%$ & $100.0 \%$ & $100.0 \%$ & $100.0 \%$ & $100.0 \%$ & $100.0 \%$ \\
\hline IRS & $0.0 \%$ & $0.0 \%$ & $0.0 \%$ & $0.0 \%$ & $0.0 \%$ & $0.0 \%$ \\
\hline DRS & $0.0 \%$ & $0.0 \%$ & $0.0 \%$ & $0.0 \%$ & $0.0 \%$ & $0.0 \%$ \\
\hline \hline
\end{tabular}


Delta Model: Difference Model by Data Analysis and Local DEA

\begin{tabular}{|c|c|c|c|c|c|c|}
\hline \multicolumn{7}{|c|}{ Altlantic Canada } \\
\hline Model/Run & CCR1 & CCR2 & CCR3 & SBM1 & SBM2 & SBM3 \\
\hline Average Efficiency & 0.467 & 0.831 & 0.829 & 0.289 & 0.758 & 0.756 \\
\hline Standard Deviation & 0.132 & 0.105 & 0.103 & 0.107 & 0.135 & 0.126 \\
\hline \multicolumn{7}{|c|}{ Quebec } \\
\hline Model/Run & CCR1 & CCR2 & CCR3 & SBM1 & SBM2 & SBM3 \\
\hline Average Efficiency & 0.535 & 0.847 & 0.861 & 0.319 & 0.759 & 0.797 \\
\hline Standard Deviation & 0.137 & 0.082 & 0.079 & 0.089 & 0.128 & 0.097 \\
\hline \multicolumn{7}{|c|}{ Northern Ontario } \\
\hline Model/Run & CCR1 & CCR2 & CCR3 & SBM1 & SBM2 & SBM3 \\
\hline Average Efficiency & 0.470 & 0.837 & 0.843 & 0.300 & 0.774 & 0.784 \\
\hline Standard Deviation & 0.108 & 0.093 & 0.093 & 0.100 & 0.118 & 0.115 \\
\hline \multicolumn{7}{|c|}{ Greater Toronto Area- Central } \\
\hline Model/Run & CCR1 & CCR2 & CCR3 & SBM1 & SBM2 & SBM3 \\
\hline Average Efficiency & 0.579 & 0.869 & 0.877 & 0.348 & 0.779 & 0.812 \\
\hline Standard Deviation & 0.173 & 0.094 & 0.089 & 0.122 & 0.138 & 0.114 \\
\hline \multicolumn{7}{|c|}{ Greater Toronto Area- West } \\
\hline Model/Run & CCR1 & CCR2 & CCR3 & SBM1 & SBM2 & SBM3 \\
\hline Average Efficiency & 0.525 & 0.844 & 0.870 & 0.322 & 0.749 & 0.799 \\
\hline Standard Deviation & 0.146 & 0.103 & 0.093 & 0.137 & 0.162 & 0.126 \\
\hline \multicolumn{7}{|c|}{ Greater Toronto Area- East } \\
\hline Model/Run & CCR1 & CCR2 & CCR3 & SBM1 & SBM2 & SBM3 \\
\hline Average Efficiency & 0.599 & 0.836 & 0.861 & 0.363 & 0.731 & 0.796 \\
\hline Standard Deviation & 0.190 & 0.093 & 0.101 & 0.113 & 0.149 & 0.129 \\
\hline \multicolumn{7}{|c|}{ Southern Ontario } \\
\hline Model/Run & CCR1 & CCR2 & CCR3 & SBM1 & SBM2 & SBM3 \\
\hline Average Efficiency & 0.451 & 0.829 & 0.831 & 0.266 & 0.755 & 0.761 \\
\hline Standard Deviation & 0.089 & 0.085 & 0.085 & 0.054 & 0.120 & 0.115 \\
\hline \multicolumn{7}{|c|}{ Prairies (Manitoba and Saskatchewan) } \\
\hline Model/Run & CCR1 & CCR2 & CCR3 & SBM1 & SBM2 & SBM3 \\
\hline Average Efficiency & 0.478 & 0.837 & 0.844 & 0.291 & 0.764 & 0.785 \\
\hline Standard Deviation & 0.134 & 0.088 & 0.088 & 0.096 & 0.118 & 0.109 \\
\hline \multicolumn{7}{|c|}{ Alberta } \\
\hline Model/Run & CCR1 & CCR2 & CCR3 & SBM1 & SBM2 & SBM3 \\
\hline Average Efficiency & 0.512 & 0.846 & 0.854 & 0.310 & 0.763 & 0.783 \\
\hline Standard Deviation & 0.144 & 0.095 & 0.093 & 0.107 & 0.140 & 0.131 \\
\hline \multicolumn{7}{|c|}{ Vancouver Lower Mainland } \\
\hline Model/Run & CCR1 & CCR2 & CCR3 & SBM1 & SBM2 & SBM3 \\
\hline Average Efficiency & 0.521 & 0.868 & 0.886 & 0.351 & 0.784 & 0.821 \\
\hline Standard Deviation & 0.136 & 0.094 & 0.083 & 0.137 & 0.142 & 0.107 \\
\hline \multicolumn{7}{|c|}{ Vancouver Island, Central BC, Northern Territories } \\
\hline Model/Run & CCR1 & CCR2 & CCR3 & SBM1 & SBM2 & SBM3 \\
\hline Average Efficiency & 0.478 & 0.855 & 0.859 & 0.288 & 0.783 & 0.791 \\
\hline Standard Deviation & 0.094 & 0.084 & 0.085 & 0.075 & 0.129 & 0.129 \\
\hline
\end{tabular}

Urban/Rural-

\begin{tabular}{|l|c|c|c|c|c|c|}
\hline \hline \multicolumn{7}{|c|}{ Urban } \\
\hline Model/Run & CCR1 & CCR2 & CCR3 & SBM1 & SBM2 & SBM3 \\
\hline Average Efficiency & 0.559 & 0.854 & 0.872 & 0.346 & 0.759 & 0.802 \\
\hline Standard Deviation & 0.161 & 0.098 & 0.091 & 0.134 & 0.152 & 0.124 \\
\hline \multicolumn{7}{|c|}{ Rural } \\
\hline Model/Run & CCR1 & CCR2 & CCR3 & SBM1 & SBM2 & SBM3 \\
\hline Average Efficiency & 0.456 & 0.835 & 0.837 & 0.273 & 0.769 & 0.774 \\
\hline Standard Deviation & 0.097 & 0.085 & 0.086 & 0.066 & 0.112 & 0.110 \\
\hline
\end{tabular}


Funds Managed-

\begin{tabular}{|c|c|c|c|c|c|c|}
\hline \multicolumn{7}{|c|}{$3,313,956.60-80,282,296.80$} \\
\hline Model/Run & CCR1 & CCR2 & CCR3 & SBM1 & SBM2 & SBM3 \\
\hline Average Efficiency & 0.460 & 0.835 & 0.838 & 0.275 & 0.771 & 0.776 \\
\hline Standard Deviation & 0.104 & 0.087 & 0.087 & 0.070 & 0.111 & 0.105 \\
\hline \multicolumn{7}{|c|}{$80,481,614.72-170,379,805.97$} \\
\hline Model/Run & CCR1 & CCR2 & CCR3 & SBM1 & SBM2 & SBM3 \\
\hline Average Efficiency & 0.537 & 0.851 & 0.862 & 0.328 & 0.759 & 0.794 \\
\hline Standard Deviation & 0.145 & 0.092 & 0.089 & 0.111 & 0.141 & 0.122 \\
\hline \multicolumn{7}{|c|}{$171,200,464.57-336,017,752.75$} \\
\hline Model/Run & CCR1 & CCR2 & CCR3 & SBM1 & SBM2 & SBM3 \\
\hline Average Efficiency & 0.640 & 0.863 & 0.902 & 0.410 & 0.746 & 0.825 \\
\hline Standard Deviation & 0.174 & 0.108 & 0.086 & 0.159 & 0.184 & 0.149 \\
\hline \multicolumn{7}{|c|}{$359,066,886.41-1,165,724,792.51$} \\
\hline Model/Run & CCR1 & CCR2 & CCR3 & SBM1 & SBM2 & SBM3 \\
\hline Average Efficiency & 0.704 & 0.903 & 0.971 & 0.499 & 0.751 & 0.892 \\
\hline Standard Deviation & 0.220 & 0.134 & 0.041 & 0.276 & 0.253 & 0.119 \\
\hline
\end{tabular}

Existing Customer Base-

\begin{tabular}{|c|c|c|c|c|c|c|}
\hline \hline \multicolumn{7}{|c|}{$342-4,092$ Customers } \\
\hline Model/Run & CCR1 & CCR2 & CCR3 & SBM1 & SBM2 & SBM3 \\
\hline Average Efficiency & 0.445 & 0.830 & 0.832 & 0.267 & 0.769 & 0.772 \\
\hline Standard Deviation & 0.092 & 0.085 & 0.084 & 0.069 & 0.107 & 0.103 \\
\hline \multicolumn{7}{|c|}{$4,117-8,038$ Customers } \\
\hline Model/Run & CCR1 & CCR2 & CCR3 & SBM1 & SBM2 & SBM3 \\
\hline Average Efficiency & 0.509 & 0.851 & 0.858 & 0.309 & 0.776 & 0.794 \\
\hline Standard Deviation & 0.119 & 0.091 & 0.090 & 0.085 & 0.126 & 0.114 \\
\hline \multicolumn{7}{|c|}{$8,186-14,314$ Customers } \\
\hline Model/Run & CCR1 & CCR2 & CCR3 & SBM1 & SBM2 & SBM3 \\
\hline Average Efficiency & 0.625 & 0.857 & 0.885 & 0.393 & 0.737 & 0.805 \\
\hline Standard Deviation & 0.177 & 0.102 & 0.089 & 0.150 & 0.172 & 0.143 \\
\hline Model/Run & $14,524-37,198$ Customers & & \\
\hline Average Efficiency & CCR1 & CCR2 & CCR3 & SBM1 & SBM2 & SBM3 \\
\hline Standard Deviation & 0.688 & 0.877 & 0.938 & 0.443 & 0.714 & 0.845 \\
\hline
\end{tabular}

\section{Returns to Scale-}

\begin{tabular}{|c|c|c|c|c|c|c|}
\hline \hline \multicolumn{7}{|c|}{ All DMUs } \\
\hline Model/Run & CCR1 & CCR2 & CCR3 & SBM1 & SBM2 & SBM3 \\
\hline CRS & $100.0 \%$ & $100.0 \%$ & $100.0 \%$ & $100.0 \%$ & $100.0 \%$ & $100.0 \%$ \\
\hline IRS & $0.0 \%$ & $0.0 \%$ & $0.0 \%$ & $0.0 \%$ & $0.0 \%$ & $0.0 \%$ \\
\hline DRS & $0.0 \%$ & $0.0 \%$ & $0.0 \%$ & $0.0 \%$ & $0.0 \%$ & $0.0 \%$ \\
\hline \multicolumn{7}{|c|}{ Funds-S } \\
\hline Model/Run & CCR1 & CCR2 & CCR3 & SBM1 & SBM2 & SBM3 \\
\hline CRS & $100.0 \%$ & $100.0 \%$ & $100.0 \%$ & $100.0 \%$ & $100.0 \%$ & $100.0 \%$ \\
\hline IRS & $0.0 \%$ & $0.0 \%$ & $0.0 \%$ & $0.0 \%$ & $0.0 \%$ & $0.0 \%$ \\
\hline DRS & $0.0 \%$ & $0.0 \%$ & $0.0 \%$ & $0.0 \%$ & $0.0 \%$ & $0.0 \%$ \\
\hline \multicolumn{7}{|c|}{ Funds-M } \\
\hline Model/Run & CCR1 & CCR2 & CCR3 & SBM1 & SBM2 & SBM3 \\
\hline CRS & $100.0 \%$ & $100.0 \%$ & $100.0 \%$ & $100.0 \%$ & $100.0 \%$ & $100.0 \%$ \\
\hline IRS & $0.0 \%$ & $0.0 \%$ & $0.0 \%$ & $0.0 \%$ & $0.0 \%$ & $0.0 \%$ \\
\hline DRS & $0.0 \%$ & $0.0 \%$ & $0.0 \%$ & $0.0 \%$ & $0.0 \%$ & $0.0 \%$ \\
\hline \multicolumn{7}{|c|}{ Funds-L } \\
\hline Model/Run & CCR1 & CCR2 & CCR3 & SBM1 & SBM2 & SBM3 \\
\hline CRS & $100.0 \%$ & $100.0 \%$ & $100.0 \%$ & $100.0 \%$ & $100.0 \%$ & $100.0 \%$ \\
\hline IRS & $0.0 \%$ & $0.0 \%$ & $0.0 \%$ & $0.0 \%$ & $0.0 \%$ & $0.0 \%$ \\
\hline DRS & $0.0 \%$ & $0.0 \%$ & $0.0 \%$ & $0.0 \%$ & $0.0 \%$ & $0.0 \%$ \\
\hline \multicolumn{7}{|c|}{ Funds-XL } \\
\hline Model/Run & CCR1 & CCR2 & CCR3 & SBM1 & SBM2 & SBM3 \\
\hline CRS & $100.0 \%$ & $100.0 \%$ & $100.0 \%$ & $100.0 \%$ & $100.0 \%$ & $100.0 \%$ \\
\hline IRS & $0.0 \%$ & $0.0 \%$ & $0.0 \%$ & $0.0 \%$ & $0.0 \%$ & $0.0 \%$ \\
\hline DRS & $0.0 \%$ & $0.0 \%$ & $0.0 \%$ & $0.0 \%$ & $0.0 \%$ & $0.0 \%$ \\
\hline \hline
\end{tabular}




\section{Delta Model: Percent Model by Stripping}

Market-

\begin{tabular}{|c|c|c|c|c|c|c|}
\hline \multicolumn{7}{|c|}{ Altlantic Canada } \\
\hline Model/Run & CCR1 & CCR2 & CCR3 & CCR4 & SBM1 & SBM2 \\
\hline Average Efficiency & 0.270 & 0.366 & 0.447 & 0.485 & 0.080 & 0.315 \\
\hline Standard Deviation & 0.090 & 0.109 & 0.078 & 0.081 & 0.017 & 0.065 \\
\hline \multicolumn{7}{|c|}{ Quebec } \\
\hline Model/Run & CCR1 & CCR2 & CCR3 & CCR4 & SBM1 & SBM2 \\
\hline Average Efficiency & 0.326 & 0.440 & 0.556 & 0.594 & 0.102 & 0.397 \\
\hline Standard Deviation & 0.079 & 0.103 & 0.118 & 0.109 & 0.034 & 0.104 \\
\hline \multicolumn{7}{|c|}{ Northern Ontario } \\
\hline Model/Run & CCR1 & CCR2 & CCR3 & CCR4 & SBM1 & SBM2 \\
\hline Average Efficiency & 0.278 & 0.379 & 0.469 & 0.509 & 0.085 & 0.334 \\
\hline Standard Deviation & 0.053 & 0.072 & 0.080 & 0.088 & 0.020 & 0.080 \\
\hline \multicolumn{7}{|c|}{ Greater Toronto Area- Central } \\
\hline Model/Run & CCR1 & CCR2 & CCR3 & CCR4 & SBM1 & SBM2 \\
\hline Average Efficiency & 0.307 & 0.418 & 0.527 & 0.573 & 0.092 & 0.361 \\
\hline Standard Deviation & 0.049 & 0.069 & 0.078 & 0.087 & 0.019 & 0.075 \\
\hline \multicolumn{7}{|c|}{ Greater Toronto Area- West } \\
\hline Model/Run & CCR1 & CCR2 & CCR3 & CCR4 & SBM1 & SBM2 \\
\hline Average Efficiency & 0.329 & 0.441 & 0.536 & 0.574 & 0.114 & 0.374 \\
\hline Standard Deviation & 0.120 & 0.148 & 0.120 & 0.119 & 0.099 & 0.135 \\
\hline \multicolumn{7}{|c|}{ Greater Toronto Area- East } \\
\hline Model/Run & CCR1 & CCR2 & CCR3 & CCR4 & SBM1 & SBM2 \\
\hline Average Efficiency & 0.334 & 0.448 & 0.569 & 0.607 & 0.105 & 0.400 \\
\hline Standard Deviation & 0.089 & 0.105 & 0.122 & 0.125 & 0.055 & 0.116 \\
\hline \multicolumn{7}{|c|}{ Southern Ontario } \\
\hline Model/Run & CCR1 & CCR2 & CCR3 & CCR4 & SBM1 & SBM2 \\
\hline Average Efficiency & 0.289 & 0.393 & 0.496 & 0.538 & 0.086 & 0.339 \\
\hline Standard Deviation & 0.063 & 0.086 & 0.098 & 0.105 & 0.018 & 0.072 \\
\hline \multicolumn{7}{|c|}{ Prairies (Manitoba and Saskatchewan) } \\
\hline Model/Run & CCR1 & CCR2 & CCR3 & CCR4 & SBM1 & SBM2 \\
\hline Average Efficiency & 0.332 & 0.440 & 0.546 & 0.590 & 0.102 & 0.377 \\
\hline Standard Deviation & 0.105 & 0.110 & 0.132 & 0.137 & 0.092 & 0.125 \\
\hline \multicolumn{7}{|c|}{ Alberta } \\
\hline Model/Run & CCR1 & CCR2 & CCR3 & CCR4 & SBM1 & SBM2 \\
\hline Average Efficiency & 0.297 & 0.402 & 0.515 & 0.549 & 0.095 & 0.366 \\
\hline Standard Deviation & 0.080 & 0.112 & 0.131 & 0.126 & 0.048 & 0.140 \\
\hline \multicolumn{7}{|c|}{ Vancouver Lower Mainland } \\
\hline Model/Run & CCR1 & CCR2 & CCR3 & CCR4 & SBM1 & SBM2 \\
\hline Average Efficiency & 0.302 & 0.418 & 0.526 & 0.570 & 0.098 & 0.383 \\
\hline Standard Deviation & 0.059 & 0.082 & 0.099 & 0.113 & 0.030 & 0.119 \\
\hline \multicolumn{7}{|c|}{ Vancouver Island, Central BC, Northern Territories } \\
\hline Model/Run & CCR1 & CCR2 & CCR3 & CCR4 & SBM1 & SBM2 \\
\hline Average Efficiency & 0.295 & 0.399 & 0.504 & 0.544 & 0.088 & 0.347 \\
\hline Standard Deviation & 0.054 & 0.074 & 0.091 & 0.098 & 0.021 & 0.084 \\
\hline
\end{tabular}

Urban/Rural-

\begin{tabular}{|l|c|c|c|c|c|c|}
\hline \hline \multicolumn{7}{|c|}{ Urban } \\
\hline Model/Run & CCR1 & CCR2 & CCR3 & CCR4 & SBM1 & SBM2 \\
\hline Average Efficiency & 0.316 & 0.425 & 0.531 & 0.570 & 0.102 & 0.375 \\
\hline Standard Deviation & 0.089 & 0.111 & 0.116 & 0.115 & 0.066 & 0.124 \\
\hline \multicolumn{7}{|c|}{ Rural } \\
\hline Model/Run & CCR1 & CCR2 & CCR3 & CCR4 & SBM1 & SBM2 \\
\hline Average Efficiency & 0.296 & 0.400 & 0.505 & 0.546 & 0.088 & 0.352 \\
\hline Standard Deviation & 0.069 & 0.093 & 0.108 & 0.114 & 0.022 & 0.093 \\
\hline
\end{tabular}


Funds Managed-

\begin{tabular}{|c|c|c|c|c|c|c|}
\hline \multicolumn{7}{|c|}{$3,313,956.60-80,282,296.80$} \\
\hline Model/Run & CCR1 & CCR2 & CCR3 & CCR4 & SBM1 & SBM2 \\
\hline Average Efficiency & 0.319 & 0.430 & 0.534 & 0.574 & 0.101 & 0.377 \\
\hline Standard Deviation & 0.100 & 0.126 & 0.134 & 0.136 & 0.069 & 0.141 \\
\hline \multicolumn{7}{|c|}{$80,481,614.72-170,379,805.97$} \\
\hline Model/Run & CCR1 & CCR2 & CCR3 & CCR4 & SBM1 & SBM2 \\
\hline Average Efficiency & 0.294 & 0.398 & 0.504 & 0.546 & 0.090 & 0.354 \\
\hline Standard Deviation & 0.055 & 0.073 & 0.089 & 0.095 & 0.019 & 0.076 \\
\hline \multicolumn{7}{|c|}{$171,200,464.57-336,017,752.75$} \\
\hline Model/Run & CCR1 & CCR2 & CCR3 & CCR4 & SBM1 & SBM2 \\
\hline Average Efficiency & 0.295 & 0.400 & 0.507 & 0.544 & 0.088 & 0.344 \\
\hline Standard Deviation & 0.055 & 0.071 & 0.081 & 0.069 & 0.015 & 0.057 \\
\hline \multicolumn{7}{|c|}{$359,066,886.41-1,165,724,792.51$} \\
\hline Model/Run & CCR1 & CCR2 & CCR3 & CCR4 & SBM1 & SBM2 \\
\hline Average Efficiency & 0.272 & 0.368 & 0.472 & 0.510 & 0.086 & 0.337 \\
\hline Standard Deviation & 0.051 & 0.069 & 0.082 & 0.088 & 0.023 & 0.087 \\
\hline
\end{tabular}

Existing Customer Base-

\begin{tabular}{|c|c|c|c|c|c|c|}
\hline \hline \multicolumn{7}{|c|}{$342-4,092$ Customers } \\
\hline Model/Run & CCR1 & CCR2 & CCR3 & CCR4 & SBM1 & SBM2 \\
\hline Average Efficiency & 0.319 & 0.428 & 0.532 & 0.571 & 0.102 & 0.374 \\
\hline Standard Deviation & 0.104 & 0.129 & 0.138 & 0.140 & 0.076 & 0.146 \\
\hline \multicolumn{7}{|c|}{$4,117-8,038$ Customers } \\
\hline Model/Run & CCR1 & CCR2 & CCR3 & CCR4 & SBM1 & SBM2 \\
\hline Average Efficiency & 0.299 & 0.406 & 0.511 & 0.552 & 0.091 & 0.359 \\
\hline Standard Deviation & 0.066 & 0.088 & 0.099 & 0.102 & 0.021 & 0.091 \\
\hline \multicolumn{7}{|c|}{$8,186-14,314$ Customers } \\
\hline Model/Run & CCR1 & CCR2 & CCR3 & CCR4 & SBM1 & SBM2 \\
\hline Average Efficiency & 0.300 & 0.407 & 0.513 & 0.553 & 0.092 & 0.361 \\
\hline Standard Deviation & 0.056 & 0.075 & 0.090 & 0.089 & 0.018 & 0.075 \\
\hline Model/Run & $14,524-37,198$ Customers & & \\
\hline Average Efficiency & CCR1 & CCR2 & CCR3 & CCR4 & SBM1 & SBM2 \\
\hline Standard Deviation & 0.286 & 0.389 & 0.496 & 0.538 & 0.087 & 0.340 \\
\hline
\end{tabular}

\section{Returns to Scale-}

\begin{tabular}{|c|c|c|c|c|c|c|}
\hline \hline \multicolumn{7}{|c|}{ All DMUs } \\
\hline Model/Run & CCR1 & CCR2 & CCR3 & CCR4 & SBM1 & SBM2 \\
\hline CRS & $100.0 \%$ & $100.0 \%$ & $100.0 \%$ & $100.0 \%$ & $100.0 \%$ & $100.0 \%$ \\
\hline IRS & $0.0 \%$ & $0.0 \%$ & $0.0 \%$ & $0.0 \%$ & $0.0 \%$ & $0.0 \%$ \\
\hline DRS & $0.0 \%$ & $0.0 \%$ & $0.0 \%$ & $0.0 \%$ & $0.0 \%$ & $0.0 \%$ \\
\hline \multicolumn{7}{|c|}{ Funds-S } \\
\hline Model/Run & CCR1 & CCR2 & CCR3 & CCR4 & SBM1 & SBM2 \\
\hline CRS & $100.0 \%$ & $100.0 \%$ & $100.0 \%$ & $100.0 \%$ & $100.0 \%$ & $100.0 \%$ \\
\hline IRS & $0.0 \%$ & $0.0 \%$ & $0.0 \%$ & $0.0 \%$ & $0.0 \%$ & $0.0 \%$ \\
\hline DRS & $0.0 \%$ & $0.0 \%$ & $0.0 \%$ & $0.0 \%$ & $0.0 \%$ & $0.0 \%$ \\
\hline \multicolumn{7}{|c|}{ Funds-M } \\
\hline Model/Run & CCR1 & CCR2 & CCR3 & CCR4 & SBM1 & SBM2 \\
\hline CRS & $100.0 \%$ & $100.0 \%$ & $100.0 \%$ & $100.0 \%$ & $100.0 \%$ & $100.0 \%$ \\
\hline IRS & $0.0 \%$ & $0.0 \%$ & $0.0 \%$ & $0.0 \%$ & $0.0 \%$ & $0.0 \%$ \\
\hline DRS & $0.0 \%$ & $0.0 \%$ & $0.0 \%$ & $0.0 \%$ & $0.0 \%$ & $0.0 \%$ \\
\hline \multicolumn{7}{|c|}{ Funds-L } \\
\hline Model/Run & CCR1 & CCR2 & CCR3 & CCR4 & SBM1 & SBM2 \\
\hline CRS & $100.0 \%$ & $100.0 \%$ & $100.0 \%$ & $100.0 \%$ & $100.0 \%$ & $100.0 \%$ \\
\hline IRS & $0.0 \%$ & $0.0 \%$ & $0.0 \%$ & $0.0 \%$ & $0.0 \%$ & $0.0 \%$ \\
\hline DRS & $0.0 \%$ & $0.0 \%$ & $0.0 \%$ & $0.0 \%$ & $0.0 \%$ & $0.0 \%$ \\
\hline \multicolumn{7}{|c|}{ Funds-XL } \\
\hline Model/Run & CCR1 & CCR2 & CCR3 & CCR4 & SBM1 & SBM2 \\
\hline CRS & $100.0 \%$ & $100.0 \%$ & $100.0 \%$ & $100.0 \%$ & $100.0 \%$ & $100.0 \%$ \\
\hline IRS & $0.0 \%$ & $0.0 \%$ & $0.0 \%$ & $0.0 \%$ & $0.0 \%$ & $0.0 \%$ \\
\hline DRS & $0.0 \%$ & $0.0 \%$ & $0.0 \%$ & $0.0 \%$ & $0.0 \%$ & $0.0 \%$ \\
\hline \hline
\end{tabular}


Delta Model: Percent Model by Data Analysis and Local DEA

Market-

\begin{tabular}{|c|c|c|c|c|c|c|}
\hline \multicolumn{7}{|c|}{ Altlantic Canada } \\
\hline Model/Run & CCR1 & CCR2 & CCR3 & SBM1 & SBM2 & SBM3 \\
\hline Average Efficiency & 0.483 & 0.741 & 0.741 & 0.374 & 0.617 & 0.624 \\
\hline Standard Deviation & 0.113 & 0.116 & 0.110 & 0.109 & 0.111 & 0.096 \\
\hline \multicolumn{7}{|c|}{ Quebec } \\
\hline Model/Run & CCR1 & CCR2 & CCR3 & SBM1 & SBM2 & SBM3 \\
\hline Average Efficiency & 0.583 & 0.798 & 0.802 & 0.450 & 0.697 & 0.696 \\
\hline Standard Deviation & 0.111 & 0.120 & 0.118 & 0.103 & 0.128 & 0.122 \\
\hline \multicolumn{7}{|c|}{ Northern Ontario } \\
\hline Model/Run & CCR1 & CCR2 & CCR3 & SBM1 & SBM2 & SBM3 \\
\hline Average Efficiency & 0.497 & 0.773 & 0.777 & 0.392 & 0.642 & 0.653 \\
\hline Standard Deviation & 0.091 & 0.142 & 0.139 & 0.092 & 0.126 & 0.122 \\
\hline \multicolumn{7}{|c|}{ Greater Toronto Area- Central } \\
\hline Model/Run & CCR1 & CCR2 & CCR3 & SBM1 & SBM2 & SBM3 \\
\hline Average Efficiency & 0.549 & 0.773 & 0.779 & 0.426 & 0.661 & 0.666 \\
\hline Standard Deviation & 0.093 & 0.124 & 0.120 & 0.082 & 0.112 & 0.109 \\
\hline \multicolumn{7}{|c|}{ Greater Toronto Area- West } \\
\hline Model/Run & CCR1 & CCR2 & CCR3 & SBM1 & SBM2 & SBM3 \\
\hline Average Efficiency & 0.538 & 0.782 & 0.791 & 0.417 & 0.669 & 0.681 \\
\hline Standard Deviation & 0.107 & 0.114 & 0.114 & 0.104 & 0.116 & 0.120 \\
\hline \multicolumn{7}{|c|}{ Greater Toronto Area- East } \\
\hline Model/Run & CCR1 & CCR2 & CCR3 & SBM1 & SBM2 & SBM3 \\
\hline Average Efficiency & 0.577 & 0.792 & 0.798 & 0.458 & 0.667 & 0.689 \\
\hline Standard Deviation & 0.140 & 0.131 & 0.139 & 0.109 & \#DIV/0! & 0.141 \\
\hline \multicolumn{7}{|c|}{ Southern Ontario } \\
\hline Model/Run & CCR1 & CCR2 & CCR3 & SBM1 & SBM2 & SBM3 \\
\hline Average Efficiency & 0.520 & 0.782 & 0.787 & 0.395 & 0.660 & 0.668 \\
\hline Standard Deviation & 0.093 & 0.139 & 0.134 & 0.084 & 0.140 & 0.135 \\
\hline \multicolumn{7}{|c|}{ Prairies (Manitoba and Saskatchewan) } \\
\hline Model/Run & CCR1 & CCR2 & CCR3 & SBM1 & SBM2 & SBM3 \\
\hline Average Efficiency & 0.576 & 0.783 & 0.793 & 0.424 & 0.663 & 0.676 \\
\hline Standard Deviation & 0.122 & 0.140 & 0.139 & 0.115 & 0.169 & 0.173 \\
\hline \multicolumn{7}{|c|}{ Alberta } \\
\hline Model/Run & CCR1 & CCR2 & CCR3 & SBM1 & SBM2 & SBM3 \\
\hline Average Efficiency & 0.537 & 0.773 & 0.778 & 0.402 & 0.659 & 0.678 \\
\hline Standard Deviation & 0.130 & 0.123 & 0.120 & 0.141 & 0.135 & 0.125 \\
\hline \multicolumn{7}{|c|}{ Vancouver Lower Mainland } \\
\hline Model/Run & CCR1 & CCR2 & CCR3 & SBM1 & SBM2 & SBM3 \\
\hline Average Efficiency & 0.534 & 0.773 & 0.788 & 0.431 & 0.651 & 0.676 \\
\hline Standard Deviation & 0.098 & 0.136 & 0.130 & 0.095 & 0.139 & 0.127 \\
\hline \multicolumn{7}{|c|}{ Vancouver Island, Central BC, Northern Territories } \\
\hline Model/Run & CCR1 & CCR2 & CCR3 & SBM1 & SBM2 & SBM3 \\
\hline Average Efficiency & 0.522 & 0.781 & 0.787 & 0.397 & 0.666 & 0.676 \\
\hline Standard Deviation & 0.084 & 0.127 & 0.123 & 0.076 & 0.116 & 0.115 \\
\hline
\end{tabular}

Urban/Rural-

\begin{tabular}{|l|c|c|c|c|c|c|}
\hline \hline \multicolumn{7}{|c|}{ Urban } \\
\hline Model/Run & CCR1 & CCR2 & CCR3 & SBM1 & SBM2 & SBM3 \\
\hline Average Efficiency & 0.545 & 0.780 & 0.787 & 0.429 & 0.663 & 0.675 \\
\hline Standard Deviation & 0.114 & 0.126 & 0.123 & 0.117 & 0.124 & 0.122 \\
\hline \multicolumn{7}{|c|}{ Rural } \\
\hline Model/Run & CCR1 & CCR2 & CCR3 & SBM1 & SBM2 & SBM3 \\
\hline Average Efficiency & 0.536 & 0.776 & 0.782 & 0.401 & 0.656 & 0.668 \\
\hline Standard Deviation & 0.112 & 0.131 & 0.128 & 0.100 & 0.138 & 0.134 \\
\hline
\end{tabular}


Funds Managed-

\begin{tabular}{|c|c|c|c|c|c|c|}
\hline \multicolumn{7}{|c|}{$3,313,956.60-80,282,296.80$} \\
\hline Model/Run & CCR1 & CCR2 & CCR3 & SBM1 & SBM2 & SBM3 \\
\hline Average Efficiency & 0.556 & 0.780 & 0.788 & 0.422 & 0.656 & 0.674 \\
\hline Standard Deviation & 0.131 & 0.133 & 0.131 & 0.132 & 0.145 & 0.142 \\
\hline \multicolumn{7}{|c|}{$80,481,614.72-170,379,805.97$} \\
\hline Model/Run & CCR1 & CCR2 & CCR3 & SBM1 & SBM2 & SBM3 \\
\hline Average Efficiency & 0.527 & 0.774 & 0.779 & 0.411 & 0.663 & 0.669 \\
\hline Standard Deviation & 0.093 & 0.124 & 0.121 & 0.083 & 0.118 & 0.115 \\
\hline \multicolumn{7}{|c|}{$171,200,464.57-336,017,752.75$} \\
\hline Model/Run & CCR1 & CCR2 & CCR3 & SBM1 & SBM2 & SBM3 \\
\hline Average Efficiency & 0.524 & 0.782 & 0.788 & 0.408 & 0.670 & 0.670 \\
\hline Standard Deviation & 0.081 & 0.121 & 0.116 & 0.085 & 0.115 & 0.111 \\
\hline \multicolumn{7}{|c|}{$359,066,886.41-1,165,724,792.51$} \\
\hline Model/Run & CCR1 & CCR2 & CCR3 & SBM1 & SBM2 & SBM3 \\
\hline Average Efficiency & 0.491 & 0.799 & 0.807 & 0.392 & 0.644 & 0.659 \\
\hline Standard Deviation & 0.094 & 0.126 & 0.116 & 0.104 & 0.111 & 0.087 \\
\hline
\end{tabular}

Existing Customer Base-

\begin{tabular}{|c|c|c|c|c|c|c|}
\hline \hline \multicolumn{7}{|c|}{$342-4,092$ Customers } \\
\hline Model/Run & CCR1 & CCR2 & CCR3 & SBM1 & SBM2 & SBM3 \\
\hline Average Efficiency & 0.552 & 0.780 & 0.789 & 0.412 & 0.652 & 0.672 \\
\hline Standard Deviation & 0.130 & 0.134 & 0.131 & 0.128 & 0.149 & 0.147 \\
\hline \multicolumn{7}{|c|}{$4,117-8,038$ Customers } \\
\hline Model/Run & CCR1 & CCR2 & CCR3 & SBM1 & SBM2 & SBM3 \\
\hline Average Efficiency & 0.536 & 0.769 & 0.774 & 0.418 & 0.657 & 0.664 \\
\hline Standard Deviation & 0.108 & 0.125 & 0.122 & 0.104 & 0.118 & 0.115 \\
\hline \multicolumn{7}{|c|}{$8,186-14,314$ Customers } \\
\hline Model/Run & CCR1 & CCR2 & CCR3 & SBM1 & SBM2 & SBM3 \\
\hline Average Efficiency & 0.534 & 0.788 & 0.793 & 0.423 & 0.680 & 0.682 \\
\hline Standard Deviation & 0.092 & 0.122 & 0.118 & 0.092 & 0.117 & 0.113 \\
\hline Model/Run & $14,524-37,198$ Customers & & \\
\hline Average Efficiency & CCR1 & CCR2 & CCR3 & SBM1 & SBM2 & SBM3 \\
\hline Standard Deviation & 0.510 & 0.807 & 0.811 & 0.401 & 0.678 & 0.685 \\
\hline
\end{tabular}

\section{Returns to Scale-}

\begin{tabular}{|c|c|c|c|c|c|c|}
\hline \hline \multicolumn{7}{|c|}{ All DMUs } \\
\hline Model/Run & CCR1 & CCR2 & CCR3 & SBM1 & SBM2 & SBM3 \\
\hline CRS & $100.0 \%$ & $100.0 \%$ & $100.0 \%$ & $100.0 \%$ & $100.0 \%$ & $100.0 \%$ \\
\hline IRS & $0.0 \%$ & $0.0 \%$ & $0.0 \%$ & $0.0 \%$ & $0.0 \%$ & $0.0 \%$ \\
\hline DRS & $0.0 \%$ & $0.0 \%$ & $0.0 \%$ & $0.0 \%$ & $0.0 \%$ & $0.0 \%$ \\
\hline \multicolumn{7}{|c|}{ Funds-S } \\
\hline Model/Run & CCR1 & CCR2 & CCR3 & SBM1 & SBM2 & SBM3 \\
\hline CRS & $100.0 \%$ & $100.0 \%$ & $100.0 \%$ & $100.0 \%$ & $100.0 \%$ & $100.0 \%$ \\
\hline IRS & $0.0 \%$ & $0.0 \%$ & $0.0 \%$ & $0.0 \%$ & $0.0 \%$ & $0.0 \%$ \\
\hline DRS & $0.0 \%$ & $0.0 \%$ & $0.0 \%$ & $0.0 \%$ & $0.0 \%$ & $0.0 \%$ \\
\hline \multicolumn{7}{|c|}{ Funds-M } \\
\hline Model/Run & CCR1 & CCR2 & CCR3 & SBM1 & SBM2 & SBM3 \\
\hline CRS & $100.0 \%$ & $100.0 \%$ & $100.0 \%$ & $100.0 \%$ & $100.0 \%$ & $100.0 \%$ \\
\hline IRS & $0.0 \%$ & $0.0 \%$ & $0.0 \%$ & $0.0 \%$ & $0.0 \%$ & $0.0 \%$ \\
\hline DRS & $0.0 \%$ & $0.0 \%$ & $0.0 \%$ & $0.0 \%$ & $0.0 \%$ & $0.0 \%$ \\
\hline \multicolumn{7}{|c|}{ Funds-L } \\
\hline Model/Run & CCR1 & CCR2 & CCR3 & SBM1 & SBM2 & SBM3 \\
\hline CRS & $100.0 \%$ & $100.0 \%$ & $100.0 \%$ & $100.0 \%$ & $100.0 \%$ & $100.0 \%$ \\
\hline IRS & $0.0 \%$ & $0.0 \%$ & $0.0 \%$ & $0.0 \%$ & $0.0 \%$ & $0.0 \%$ \\
\hline DRS & $0.0 \%$ & $0.0 \%$ & $0.0 \%$ & $0.0 \%$ & $0.0 \%$ & $0.0 \%$ \\
\hline \multicolumn{7}{|c|}{ Funds-XL } \\
\hline Model/Run & CCR1 & CCR2 & CCR3 & SBM1 & SBM2 & SBM3 \\
\hline CRS & $100.0 \%$ & $100.0 \%$ & $100.0 \%$ & $100.0 \%$ & $100.0 \%$ & $100.0 \%$ \\
\hline IRS & $0.0 \%$ & $0.0 \%$ & $0.0 \%$ & $0.0 \%$ & $0.0 \%$ & $0.0 \%$ \\
\hline DRS & $0.0 \%$ & $0.0 \%$ & $0.0 \%$ & $0.0 \%$ & $0.0 \%$ & $0.0 \%$ \\
\hline \hline
\end{tabular}




\section{Appendix 3: Target Setting}

\section{0-2011 Period Growth Model}

CCR Model:

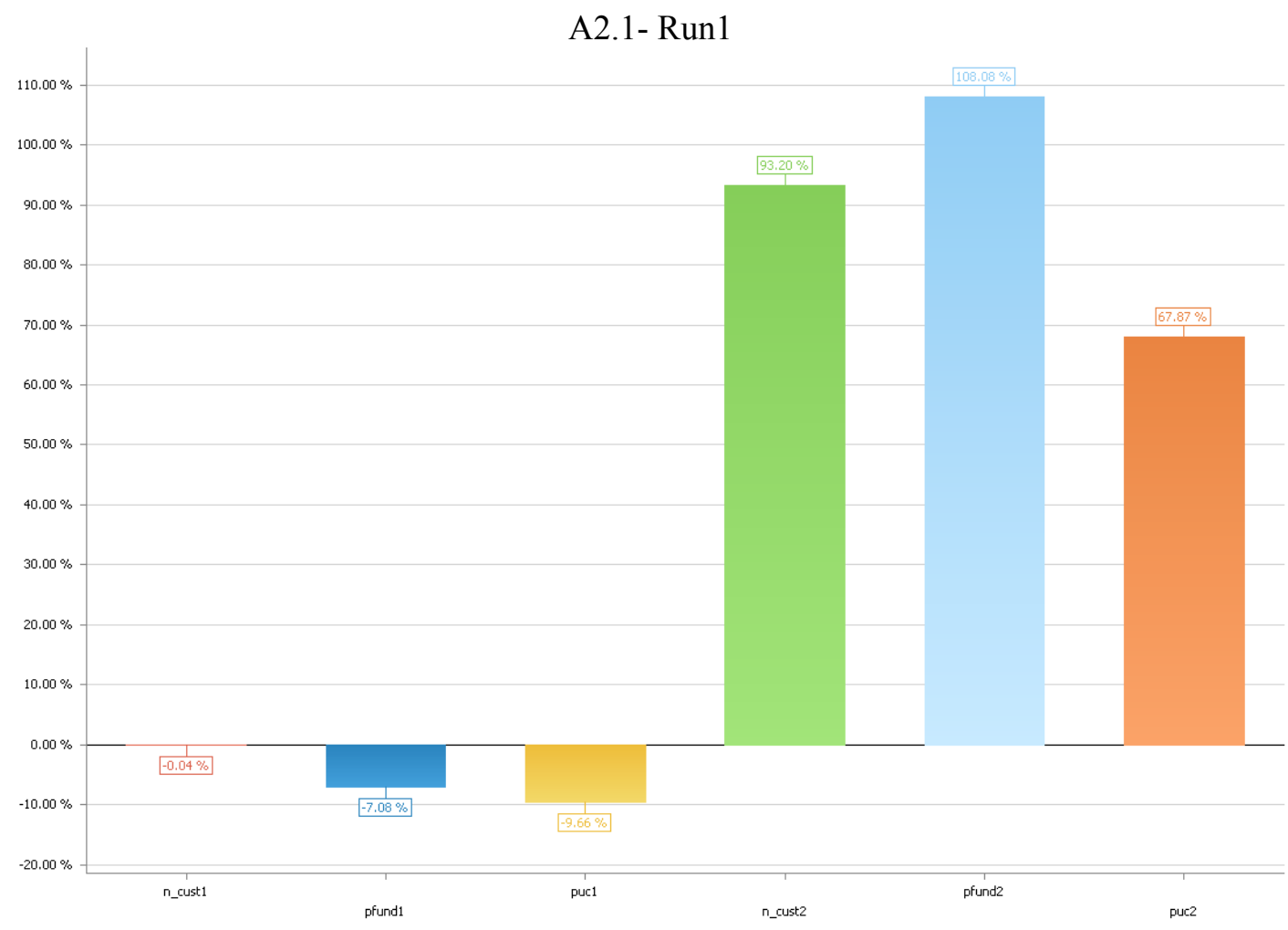

A2.2- Run2

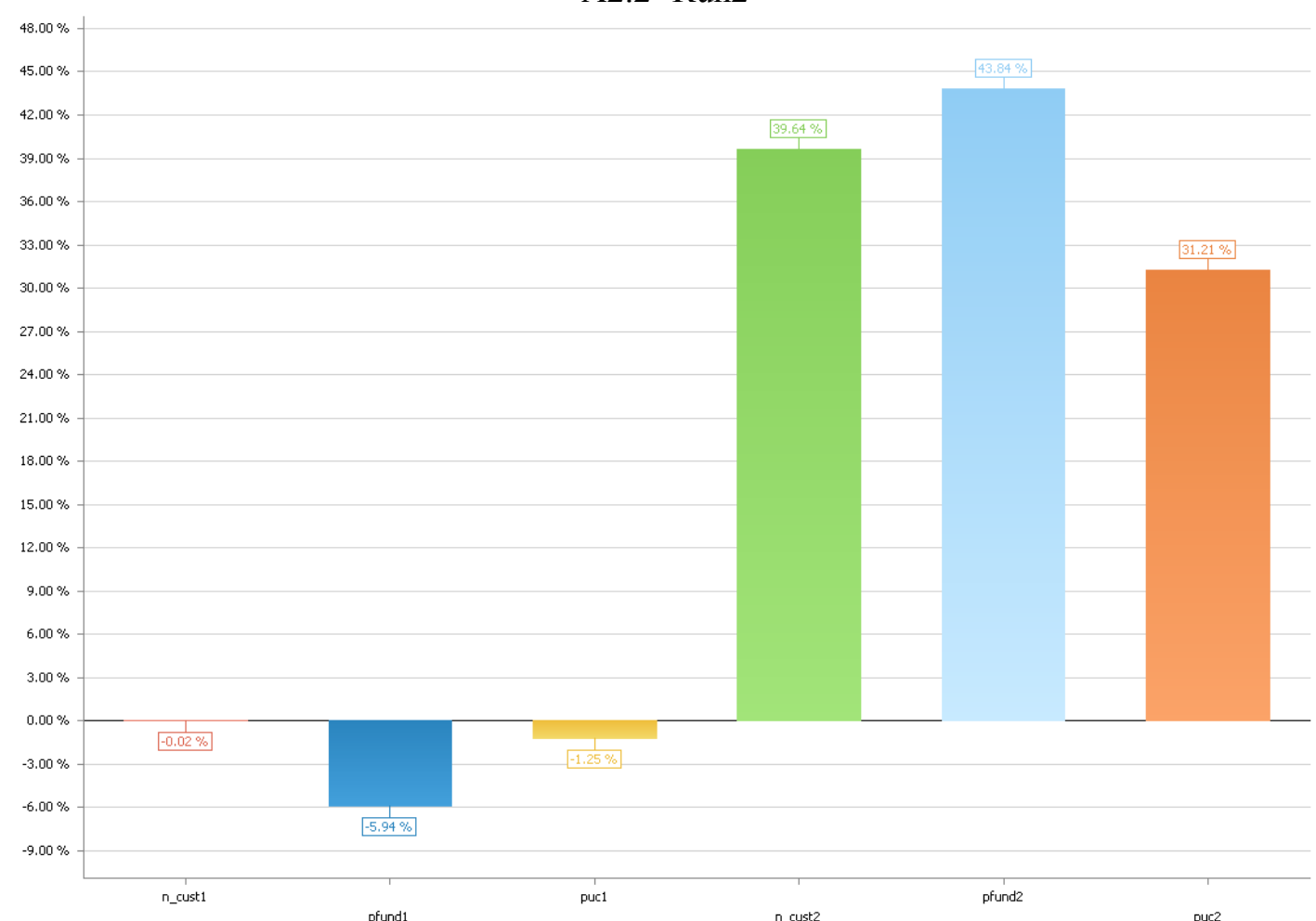

pfund

n_cust2 
A2.3- Run3

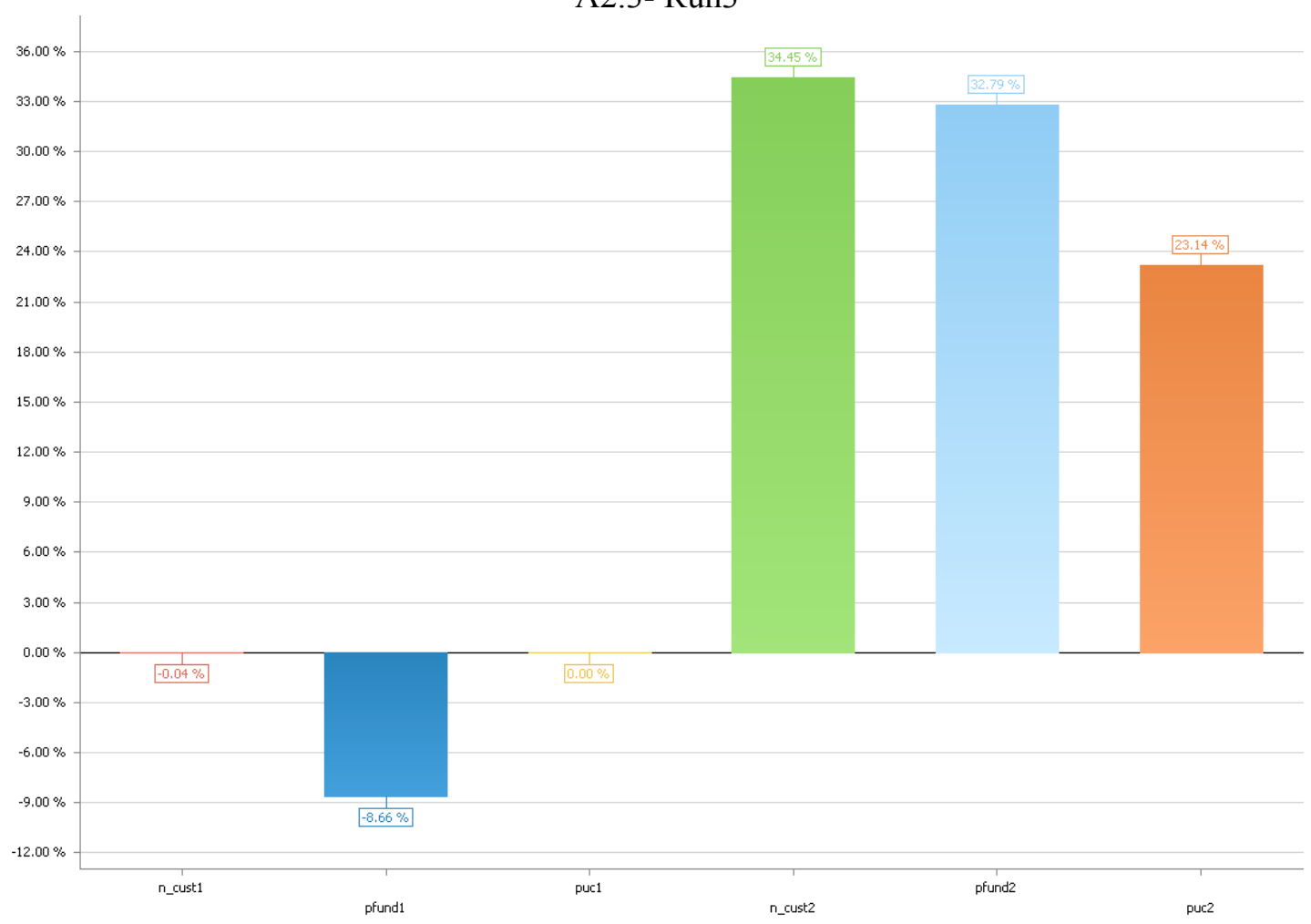

BCC Model:

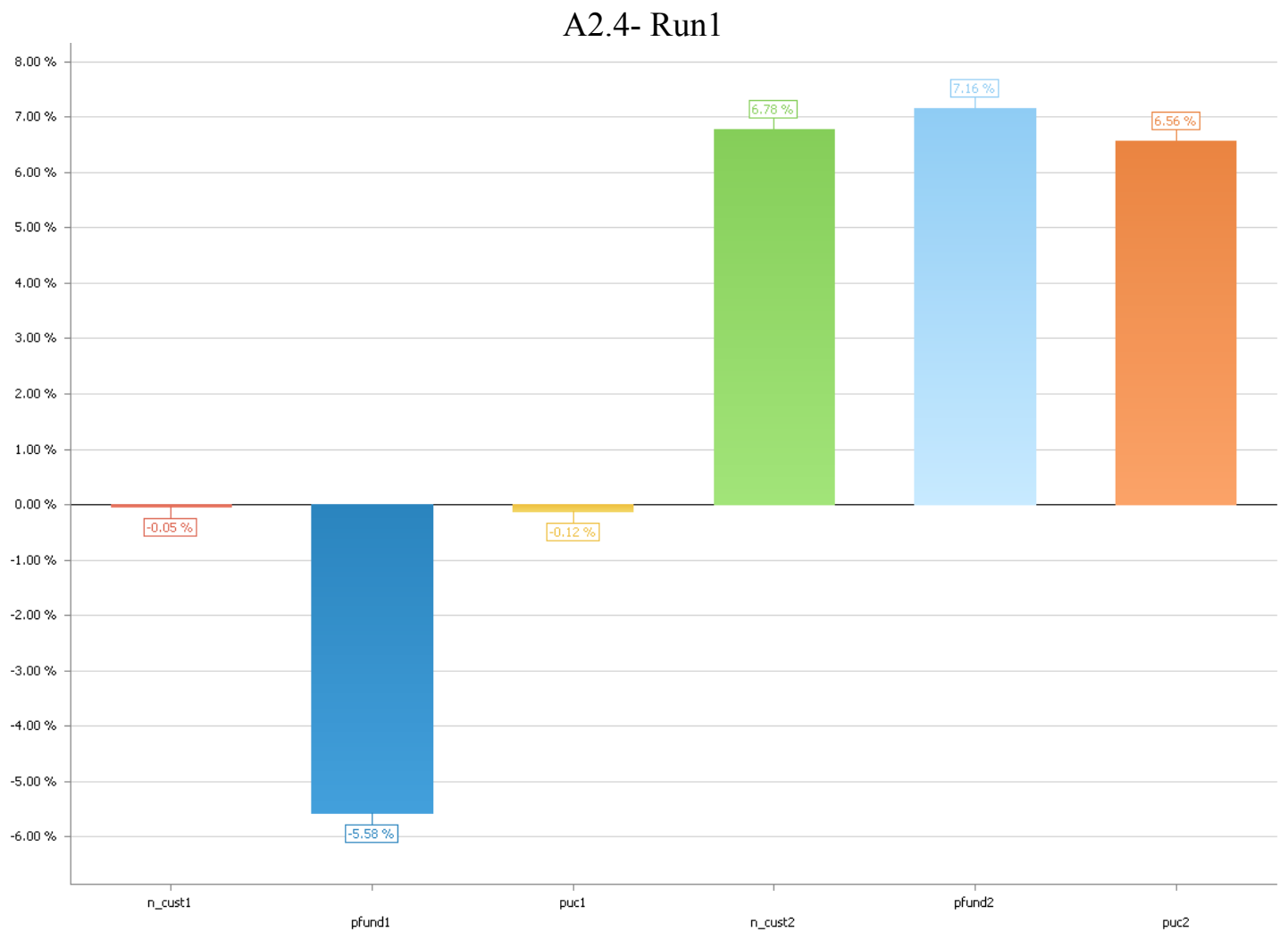




\section{Lost Accounts/Gained Accounts Model}

\section{CCR Model:}

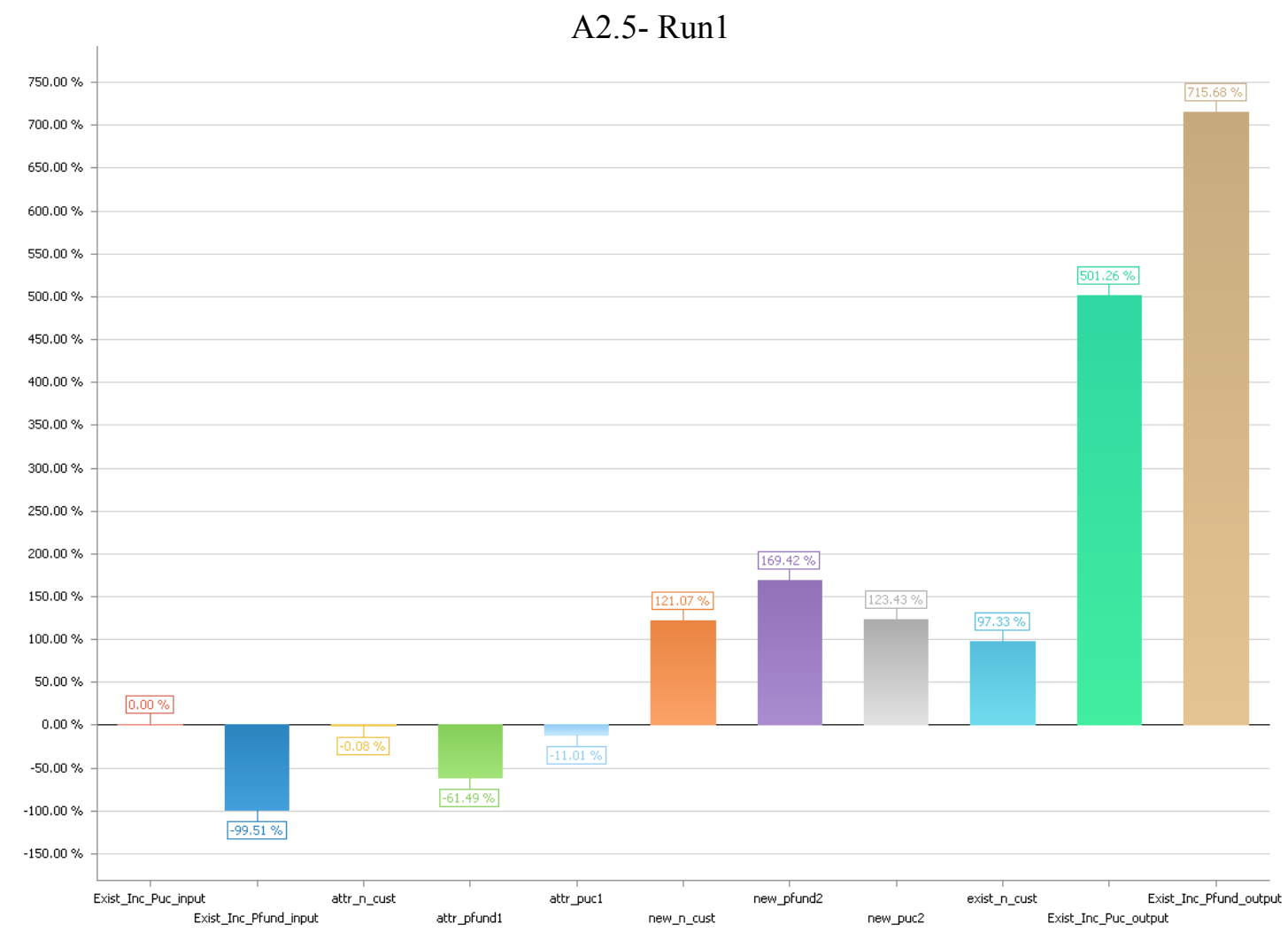

A2.6- Run2

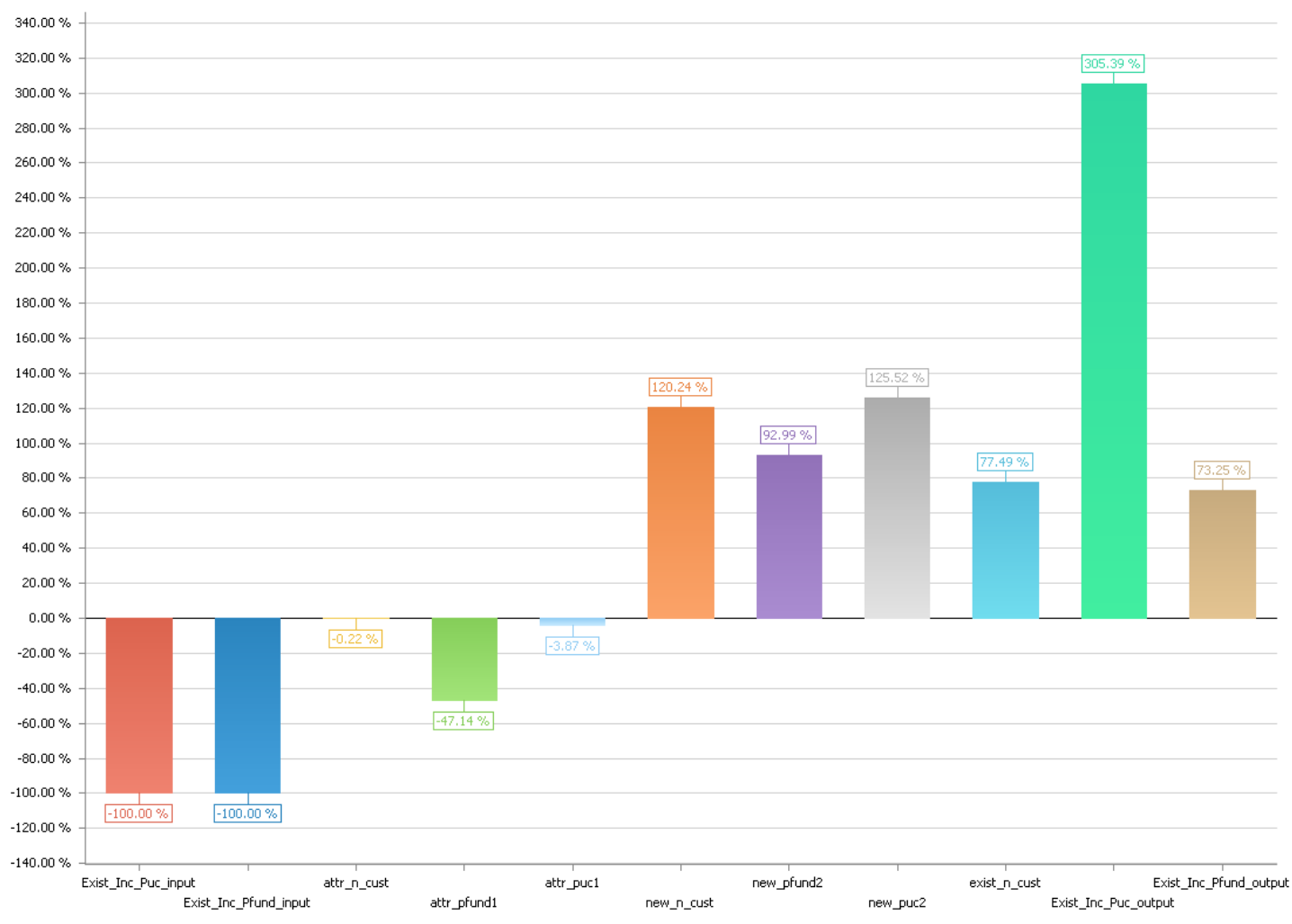


A2.7- Run3

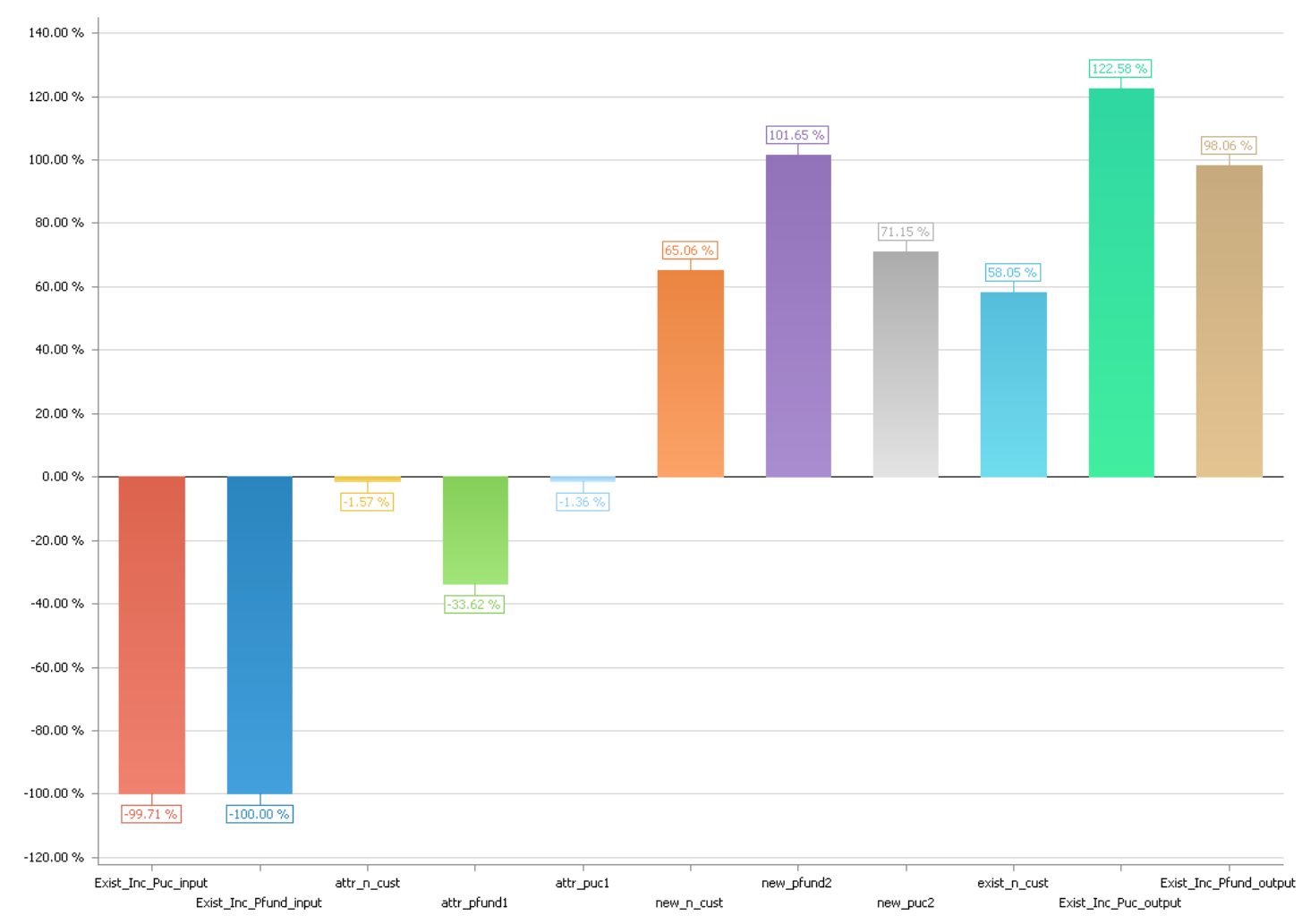

BCC Model:

A2.8-Run1

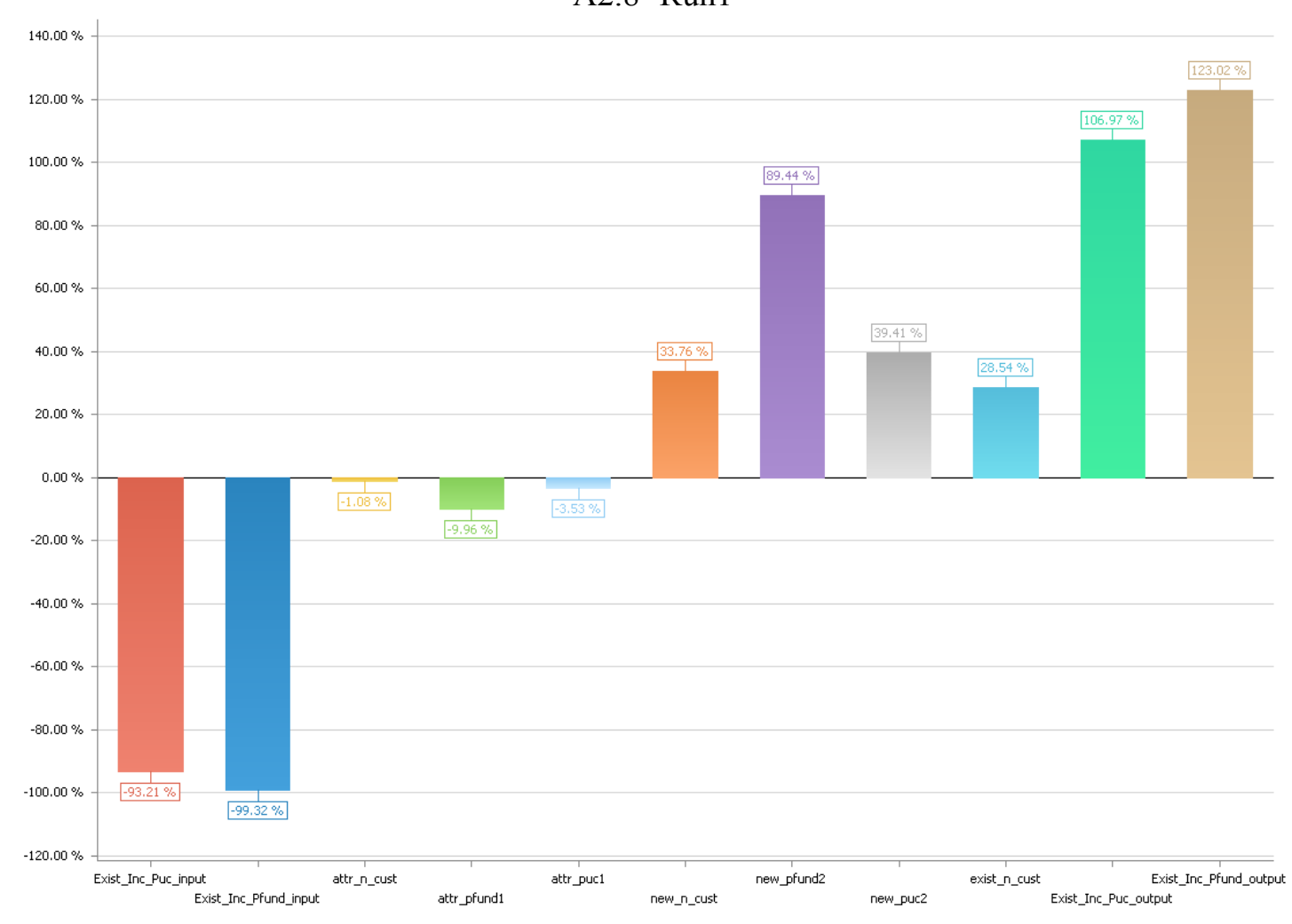




\section{Market Model: Component Model}

CCR Model:

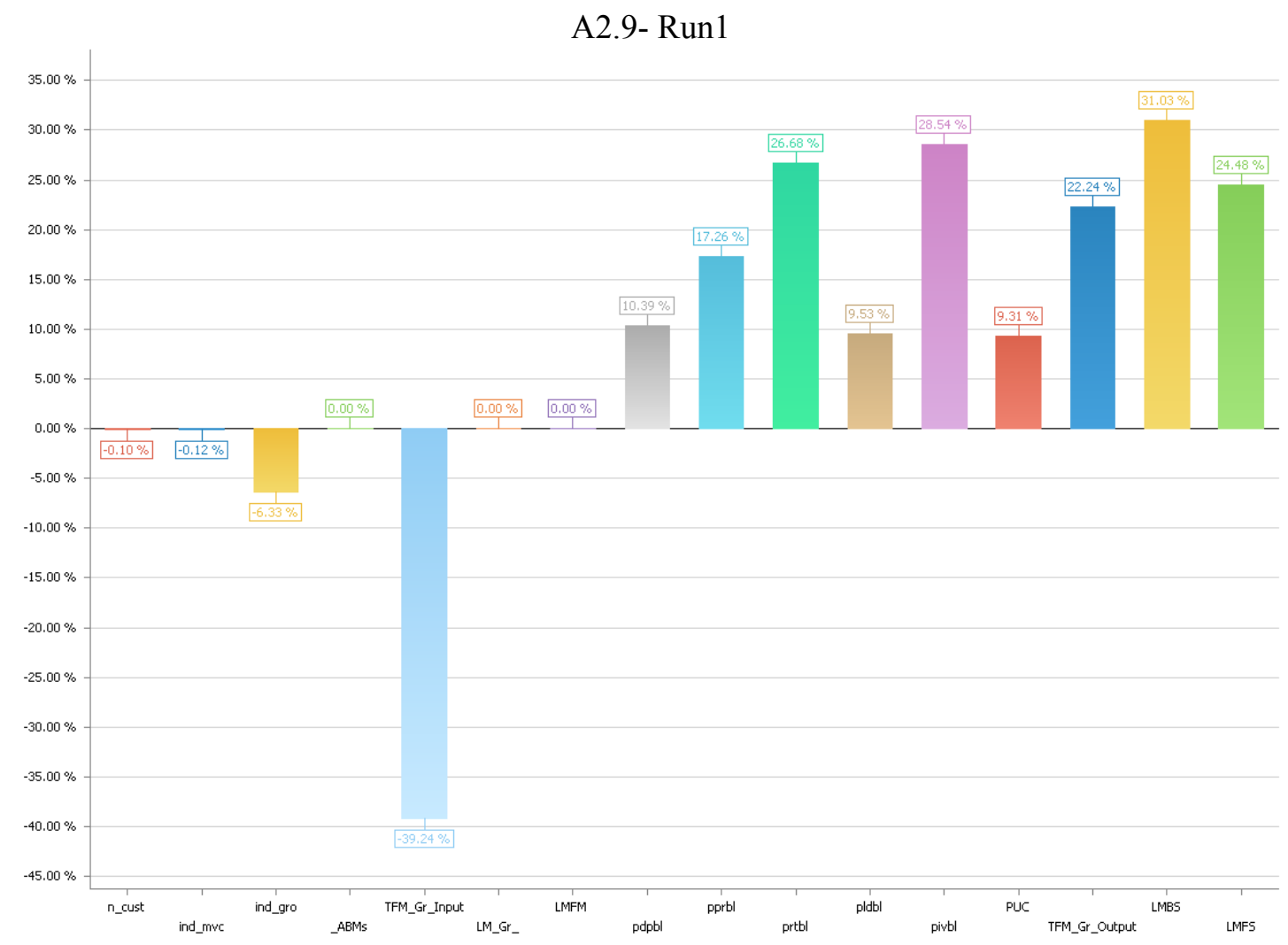

A2.10- Run3

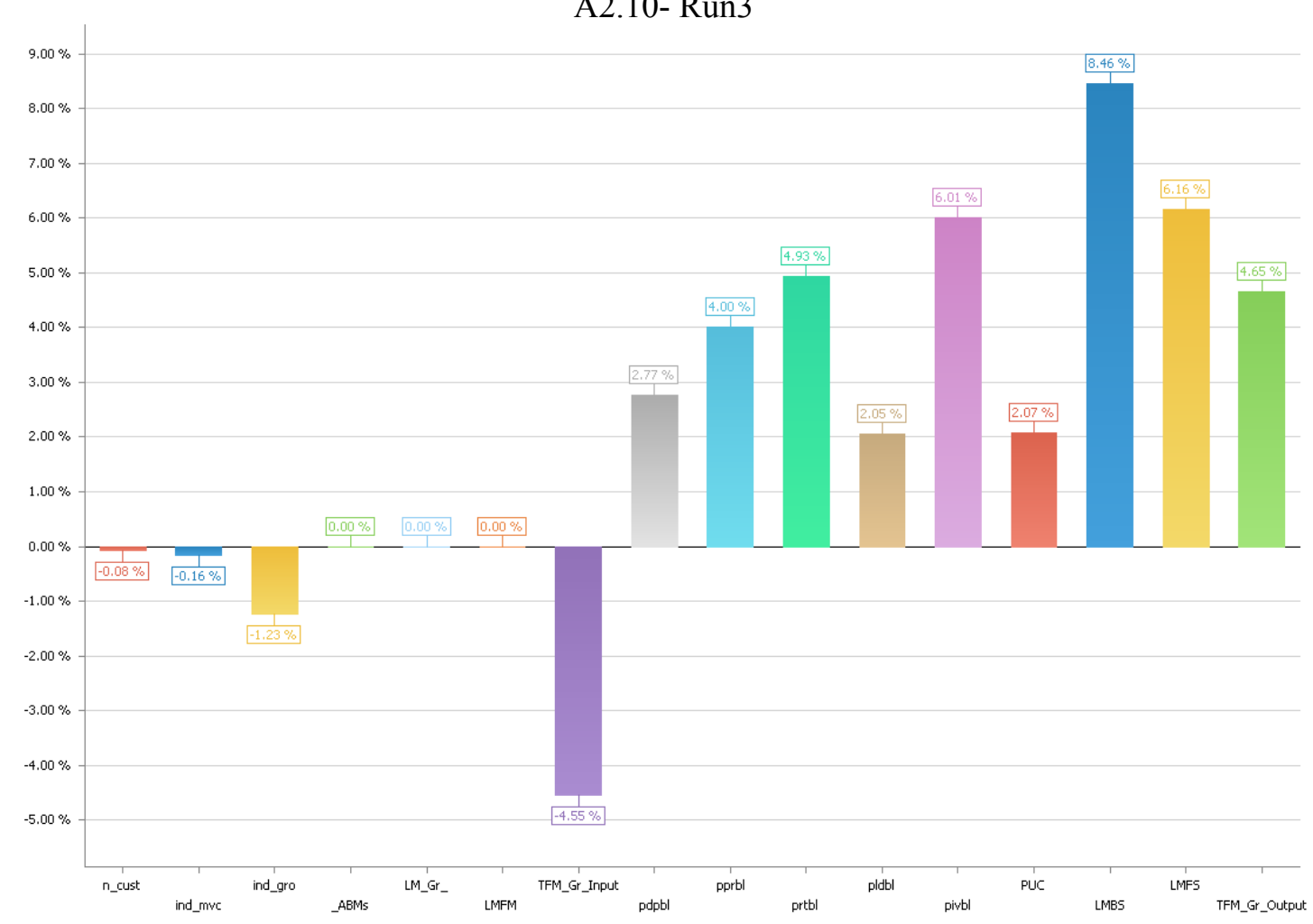




\section{BCC Model:}

A2.11- Run1

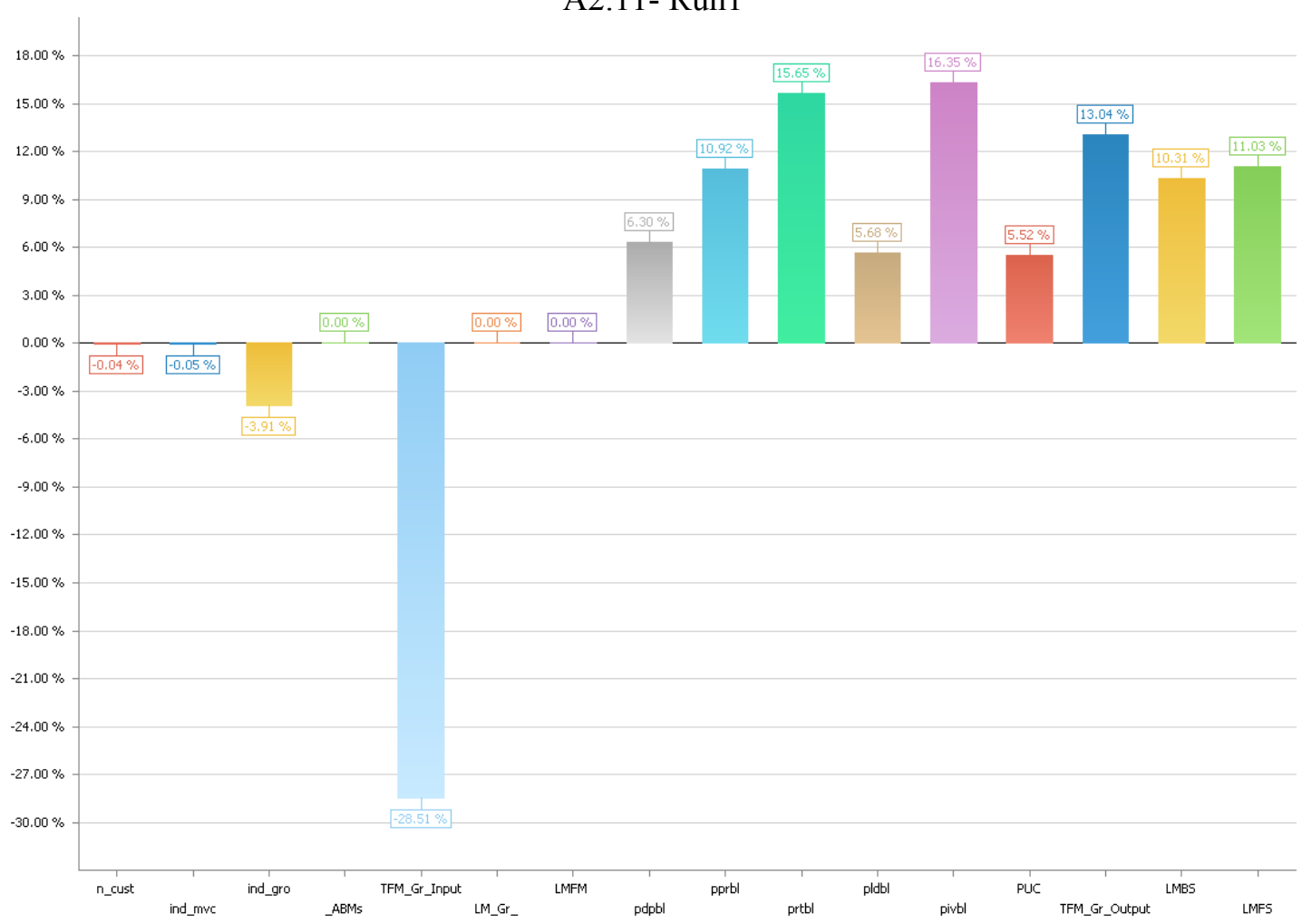

A2.12- Run3

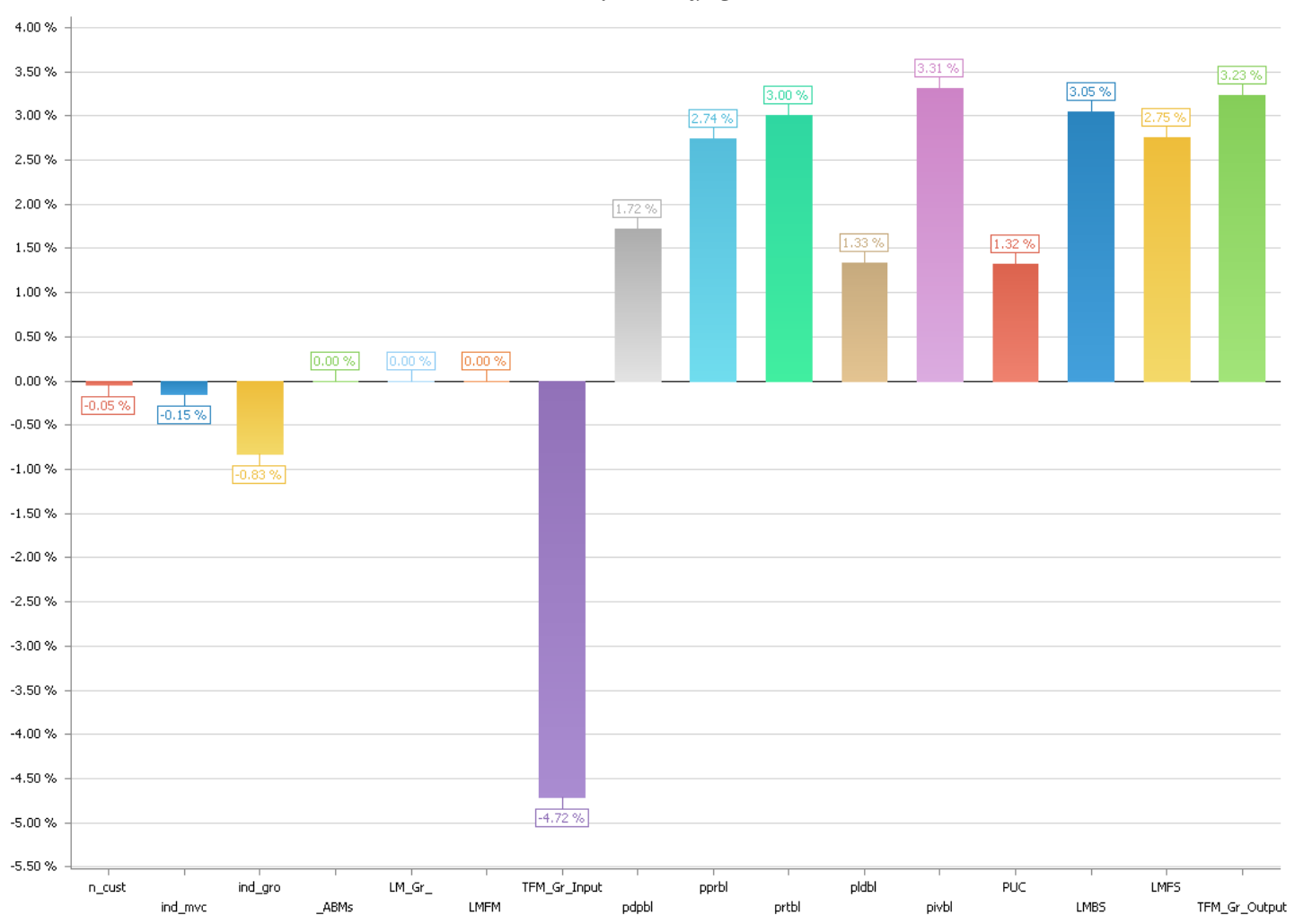




\section{Market Model: Aggregate Model}

CCR Model:

A2.13- Run1

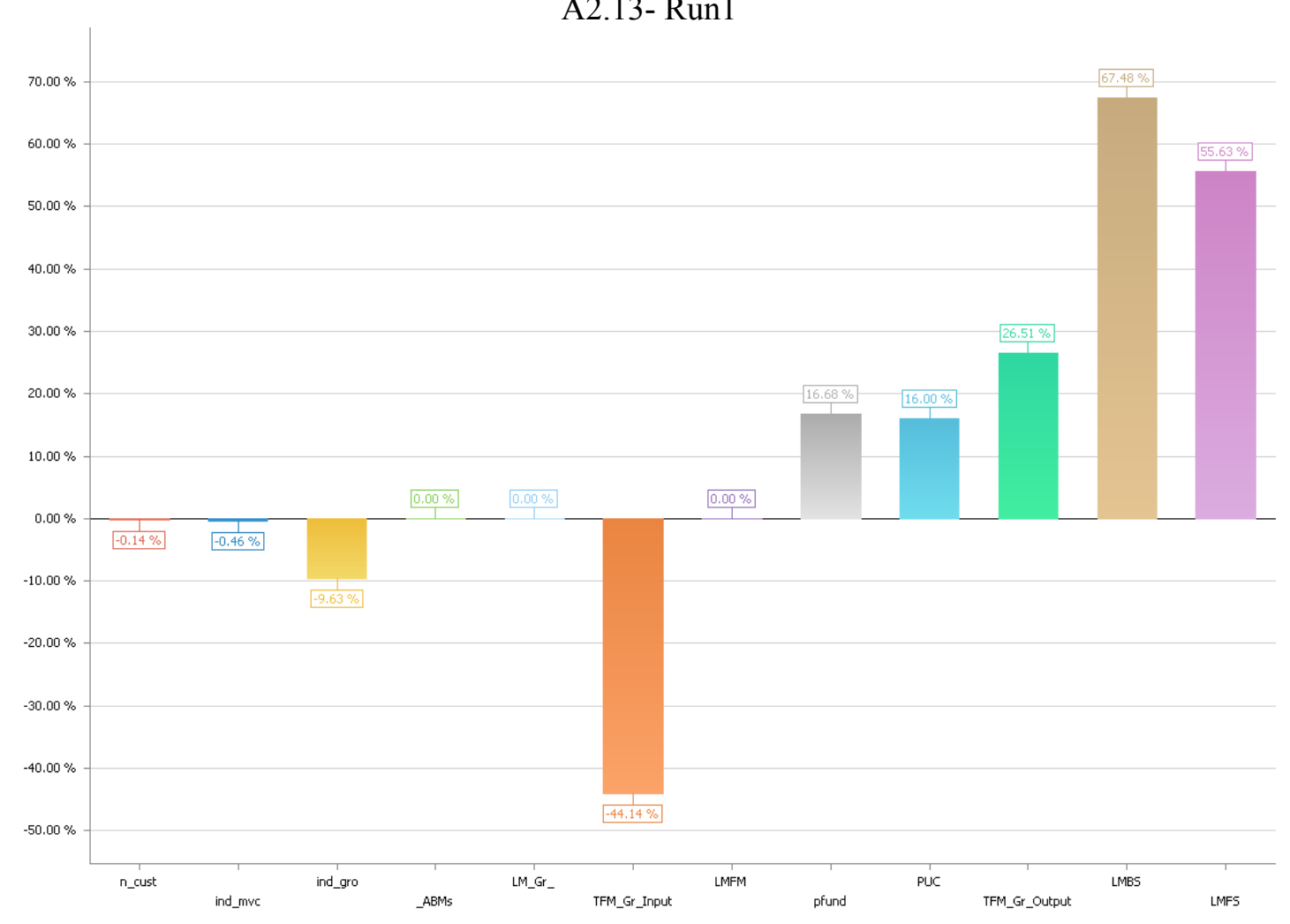

A2.14- Run3

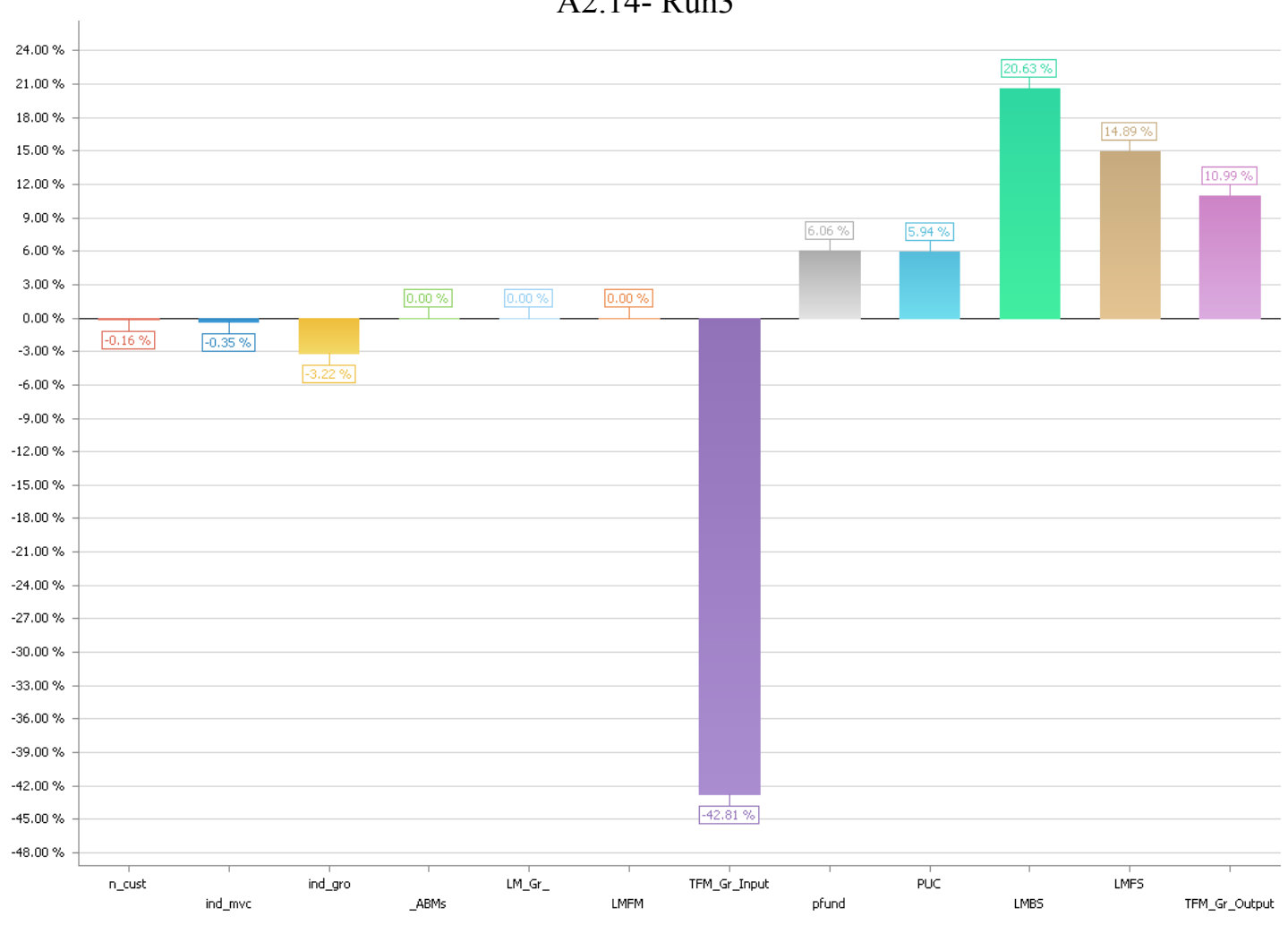




\section{BCC Model:}

\section{A2.15- Run1}

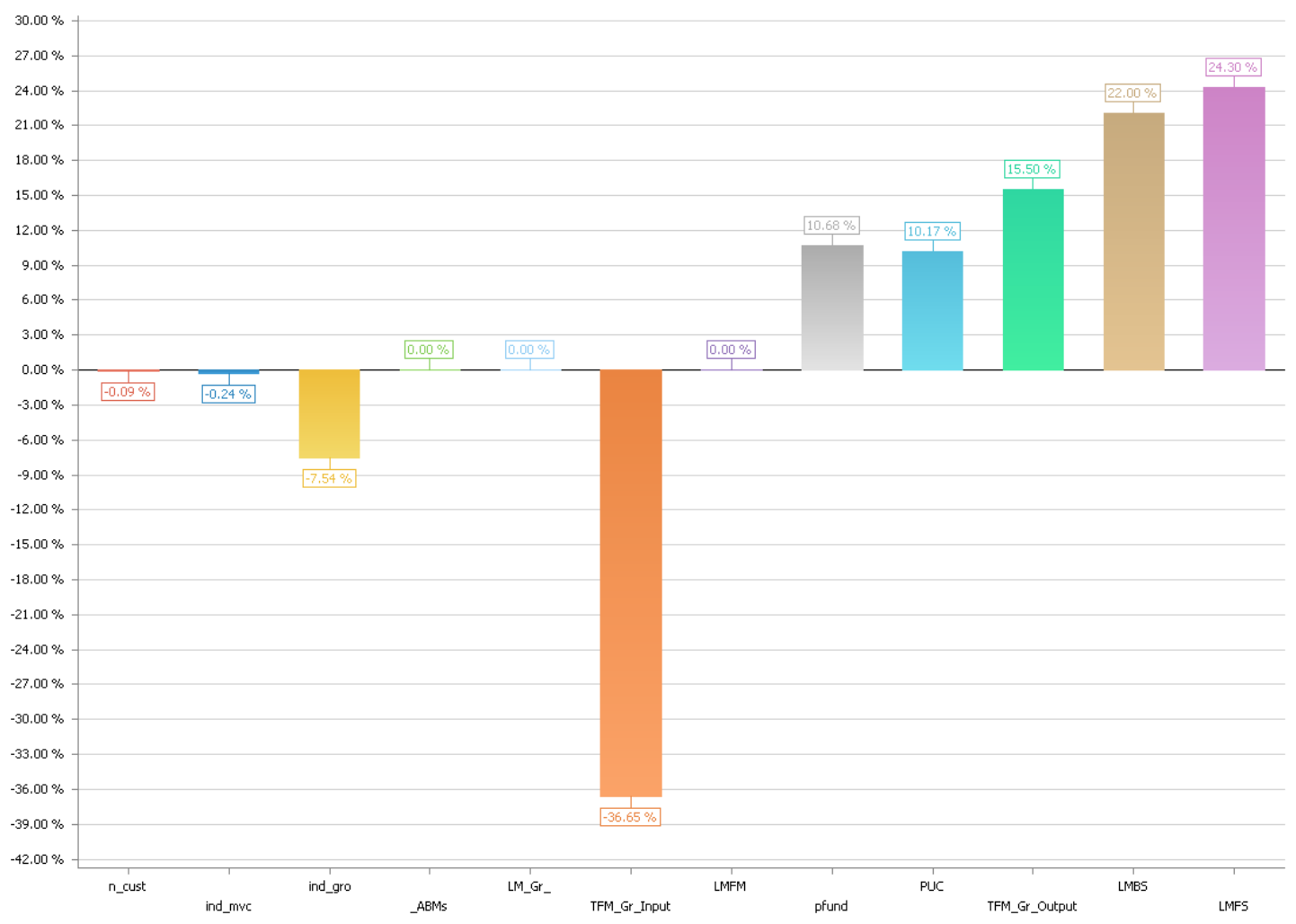

A2.16- Run3

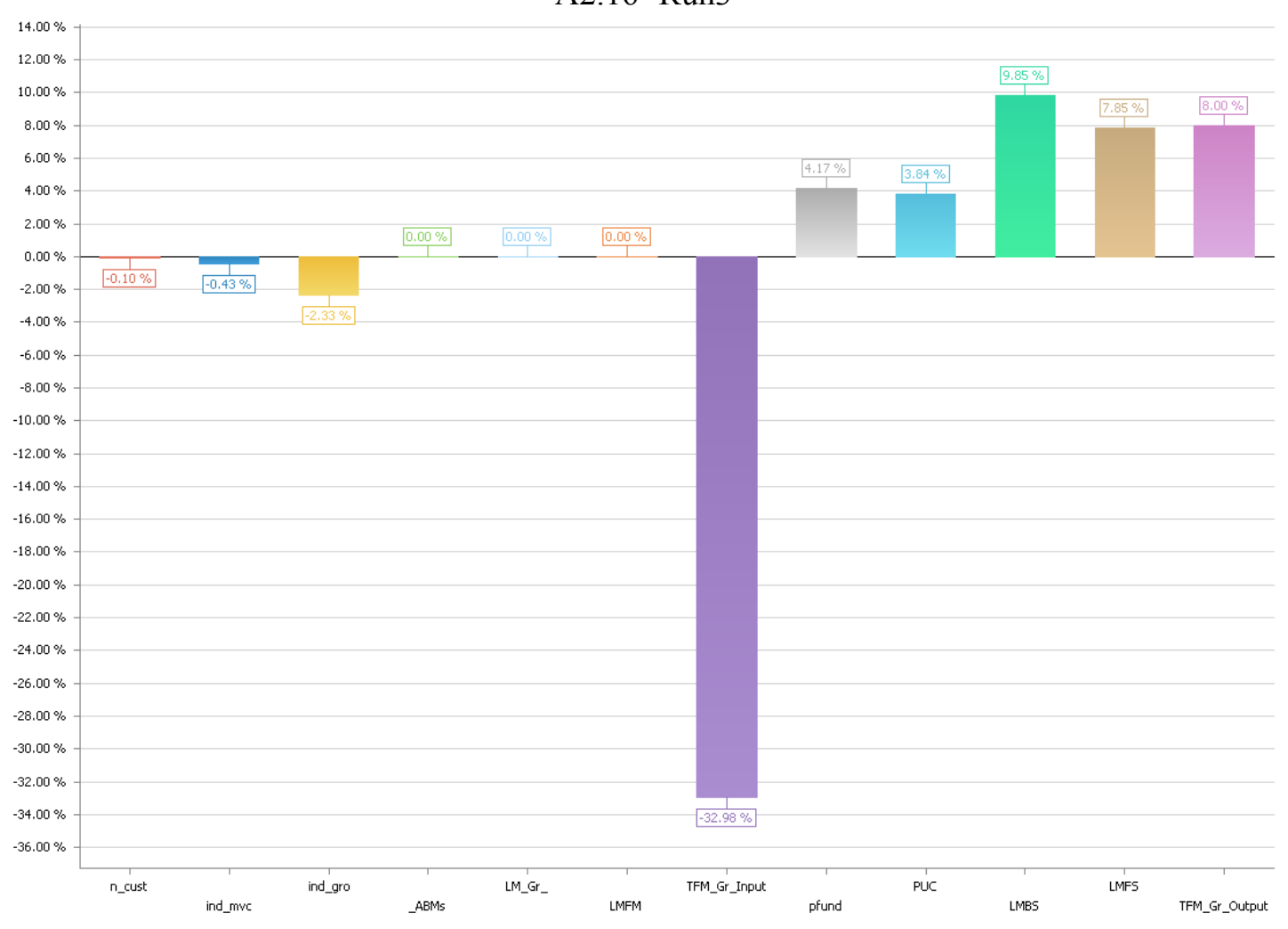




\section{Delta Model:}

\section{Difference Model: Stripping Efficient Frontier}

CCR Model:

\section{A2.17- Run1}

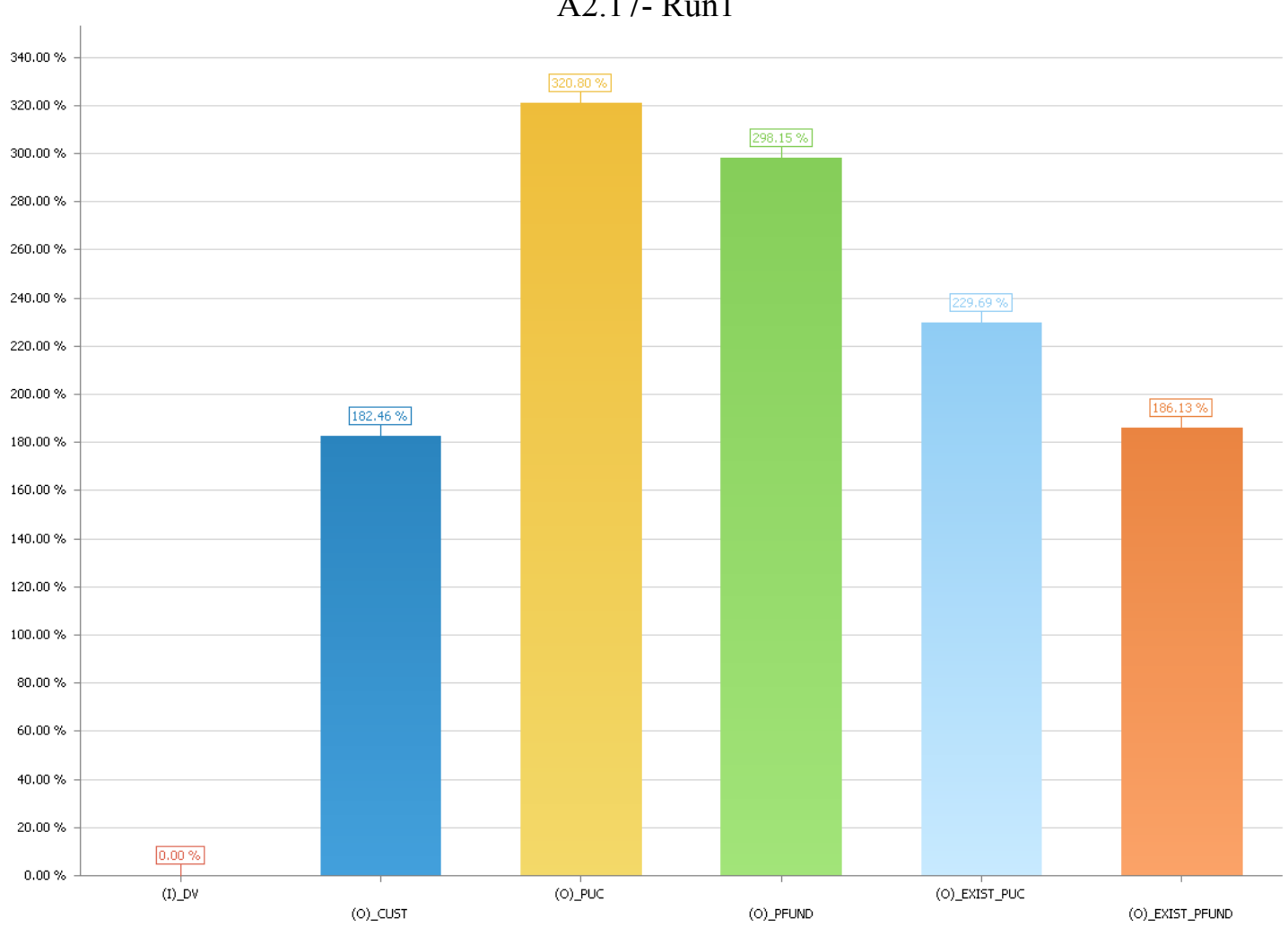

A2.18- Run2

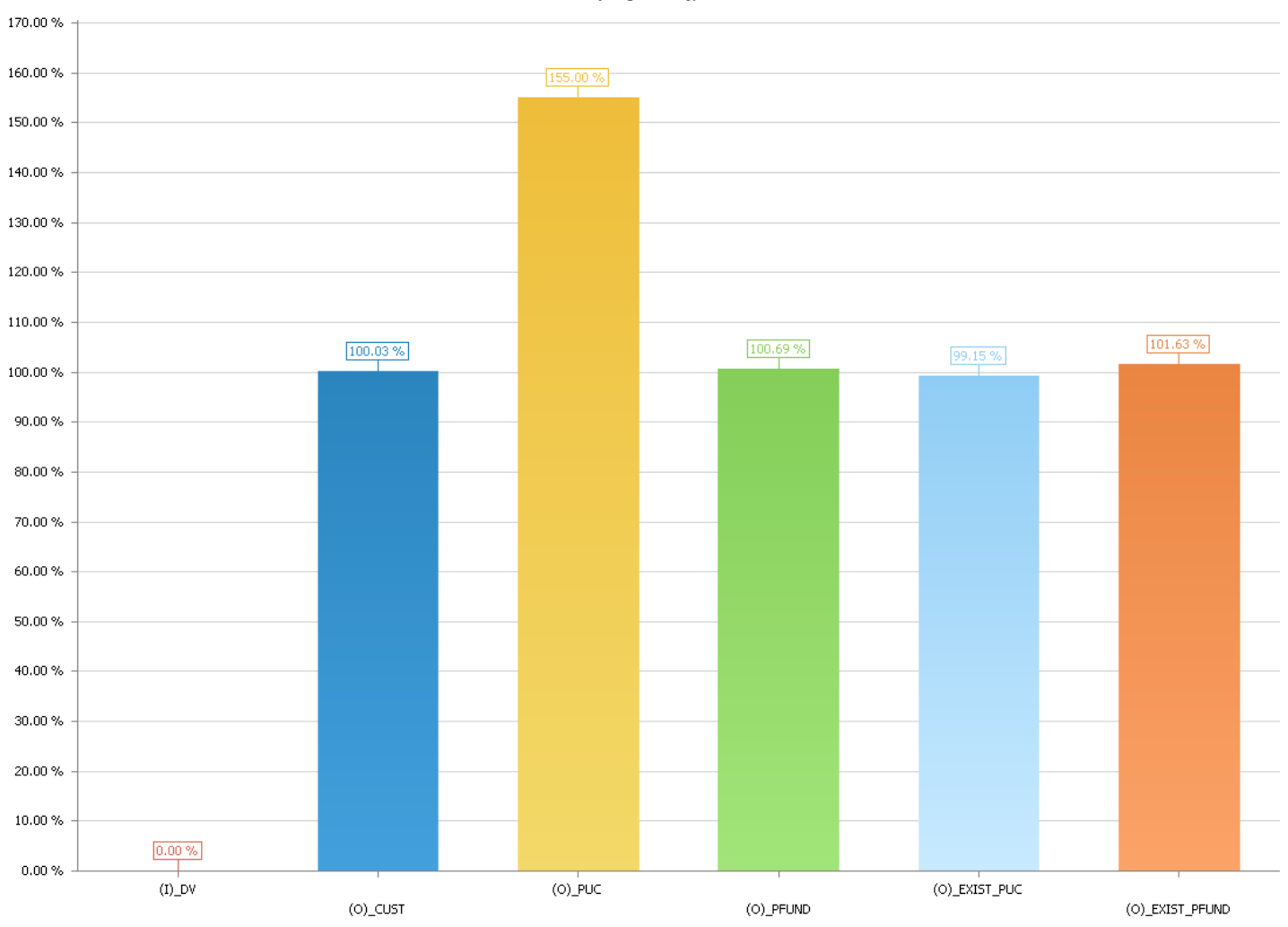

Multi-Stage Evaluation of Bank Branch Consolidation and Growth Potential Using DEA 158 


\section{A2.19- Run3}

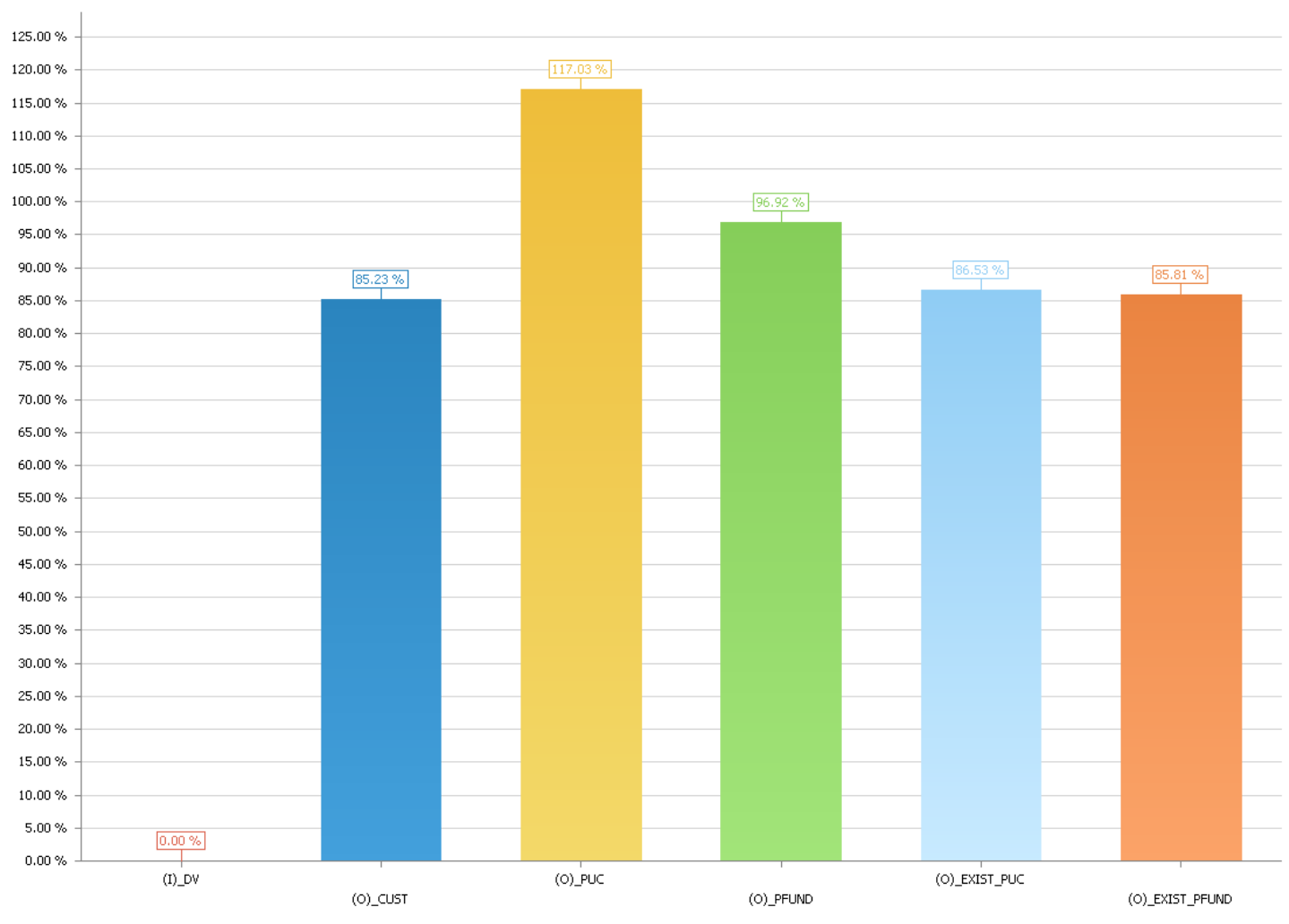

A2.20- Run 4

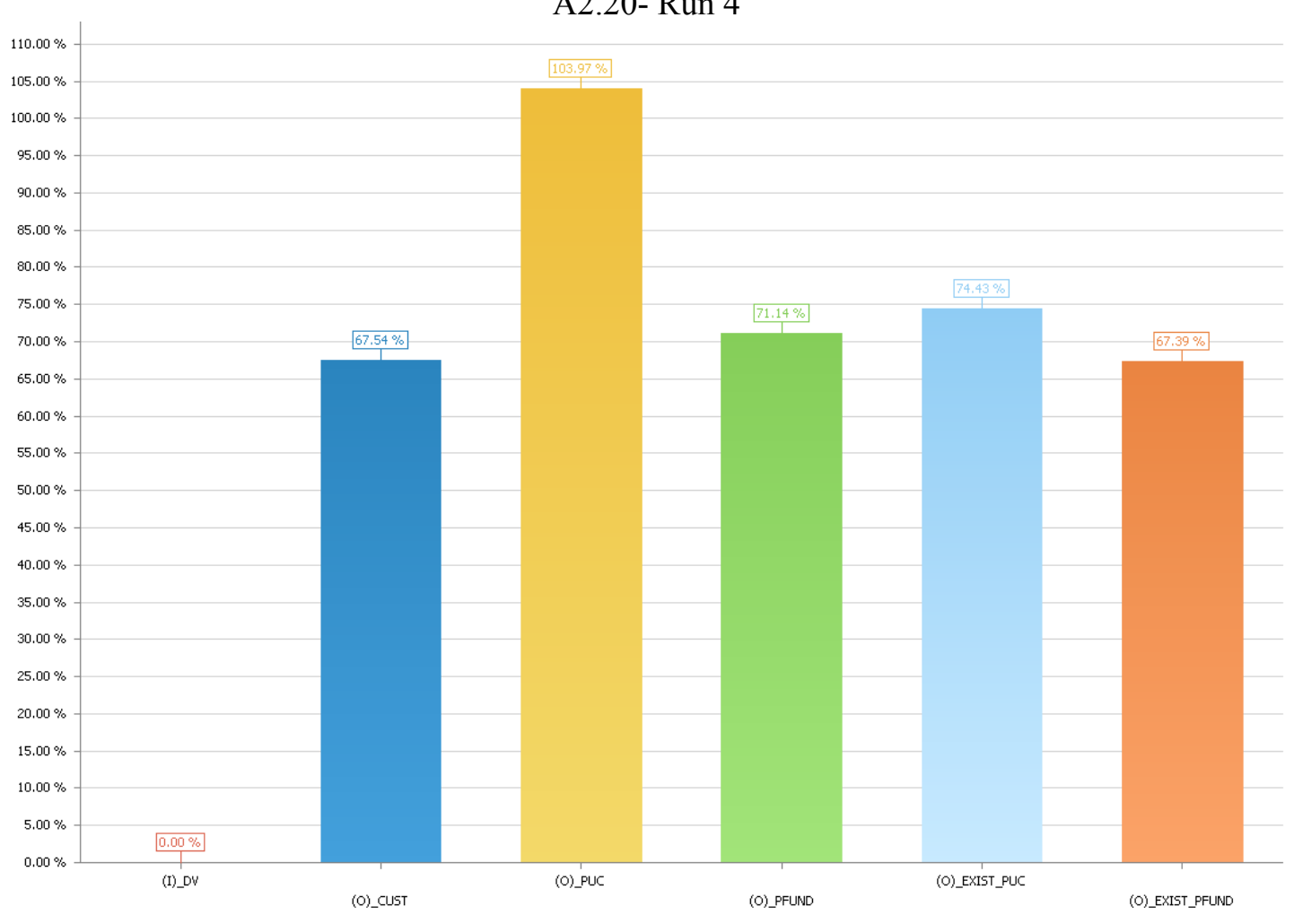


SBM Mode:

A2.21- Run 1

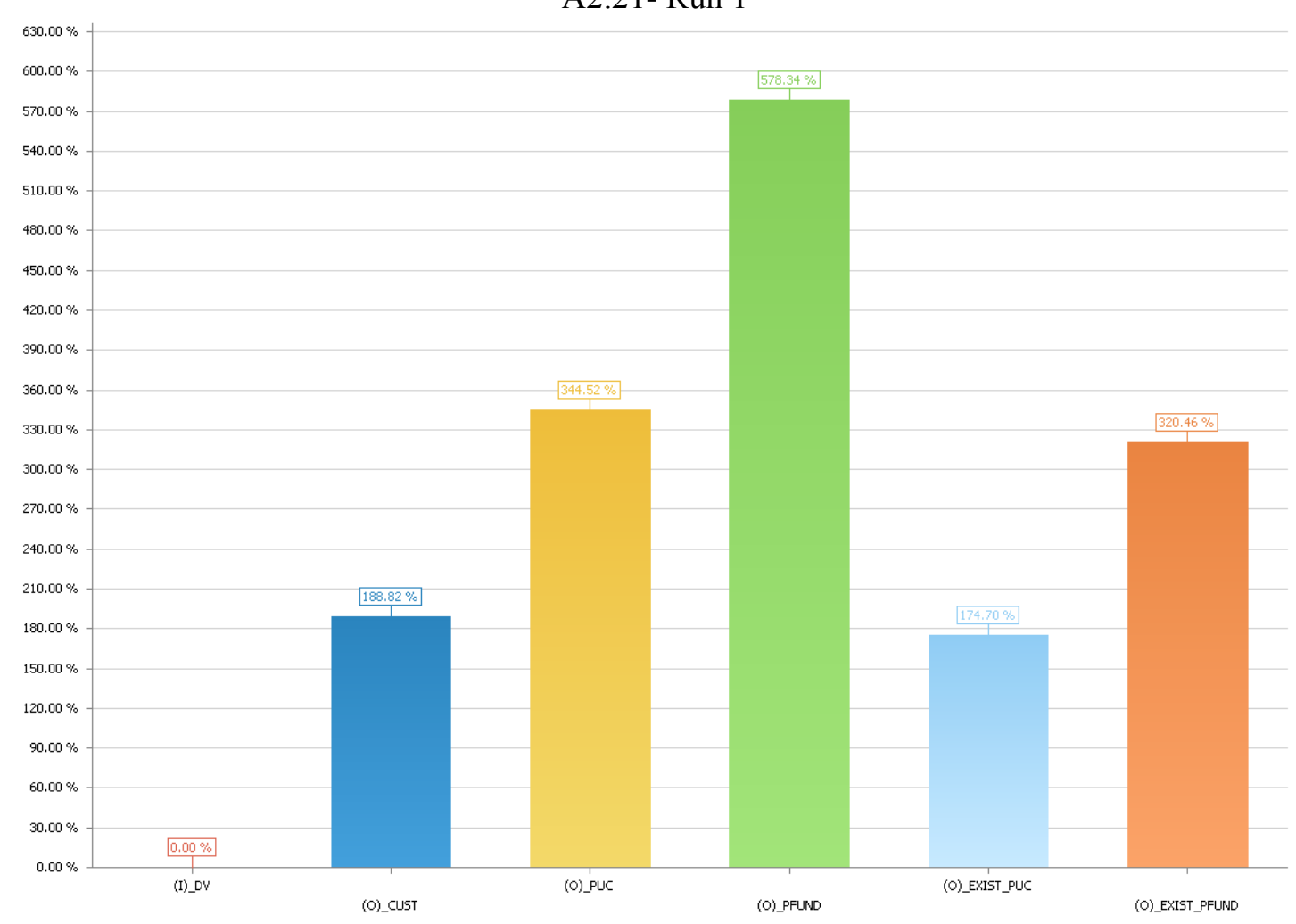

A2.22- Run 2

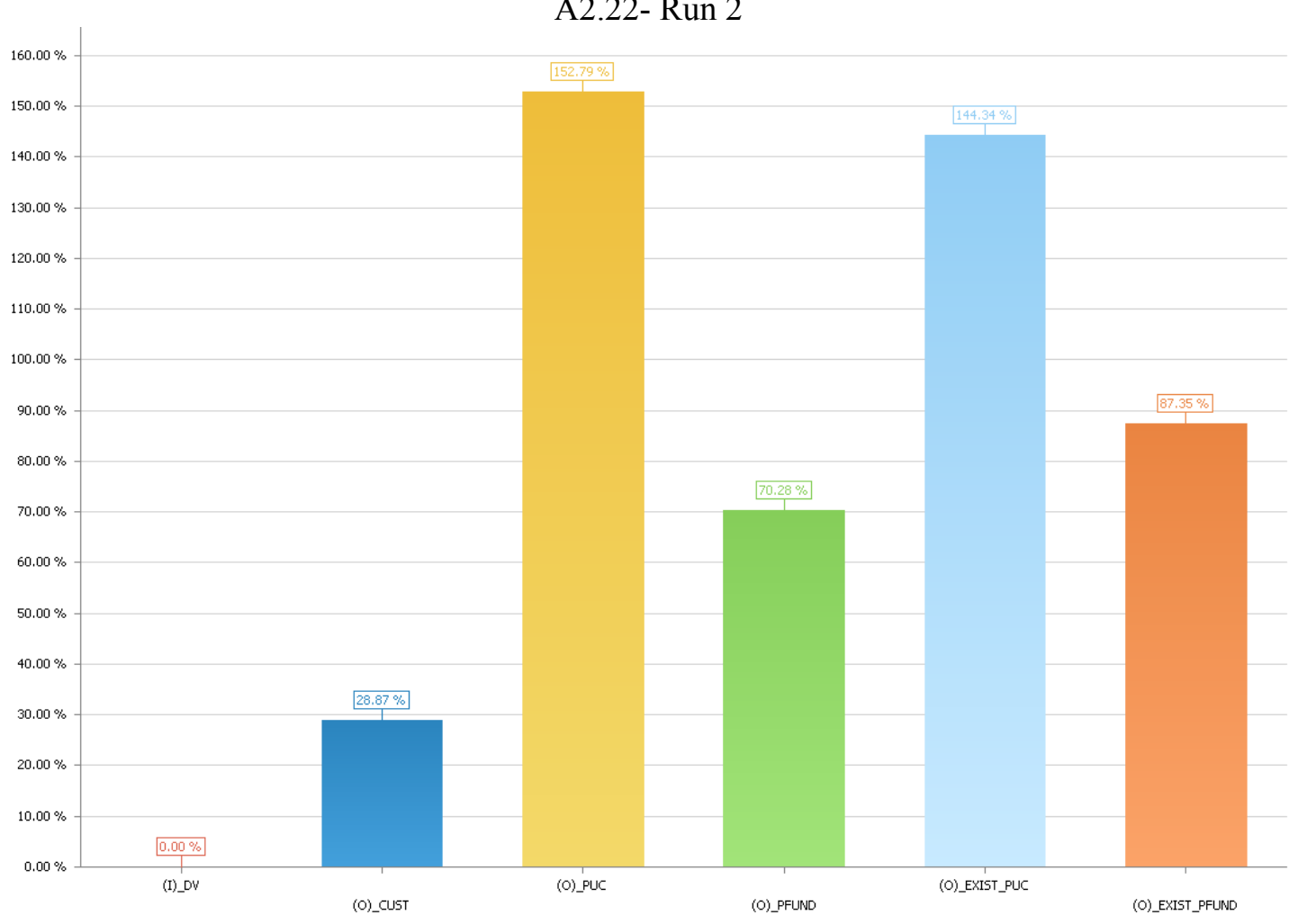




\section{Difference Model: Statistical Analysis and Local DEA}

CCR Model:

A2.23- Run1

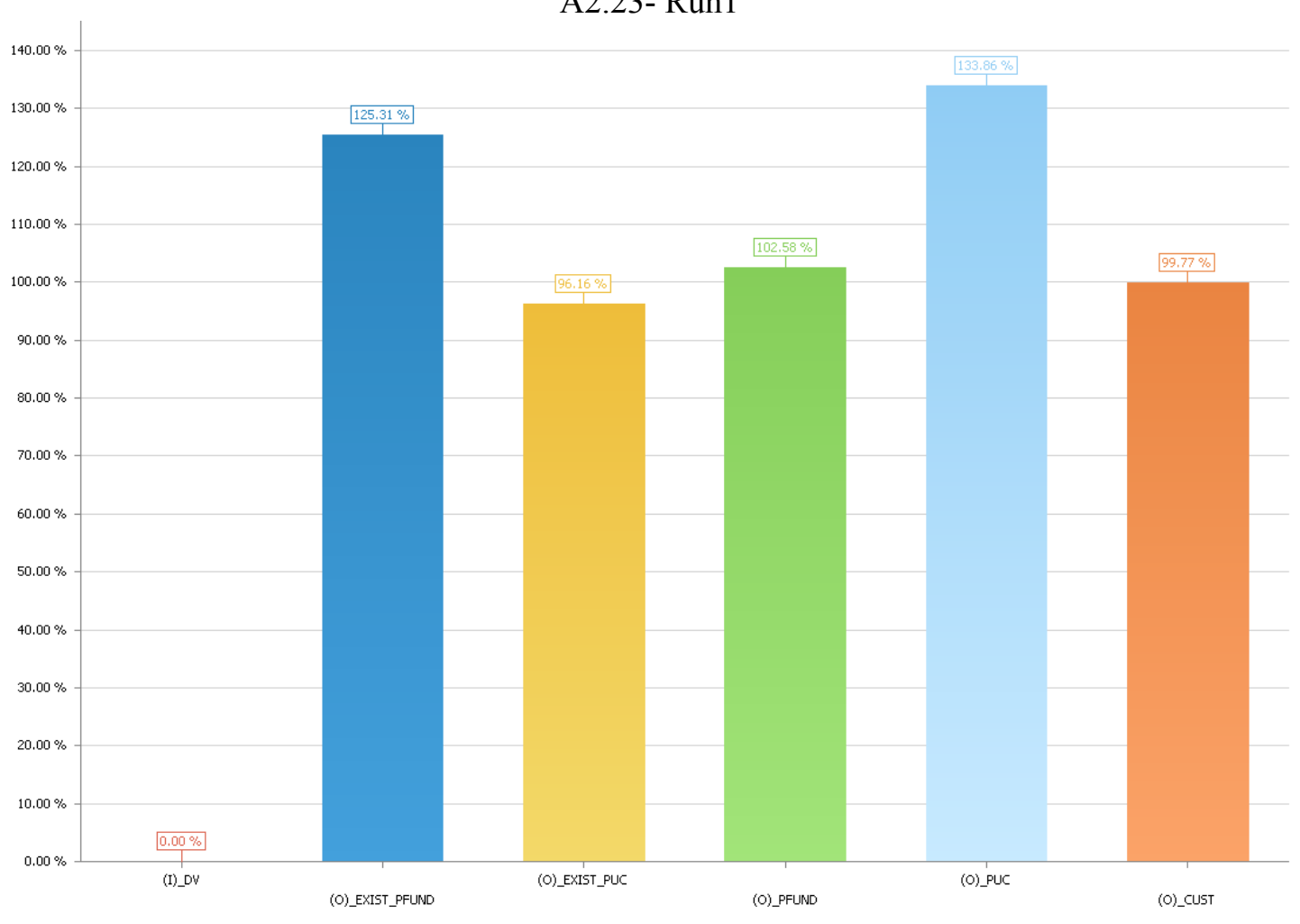

A2.24- Run2

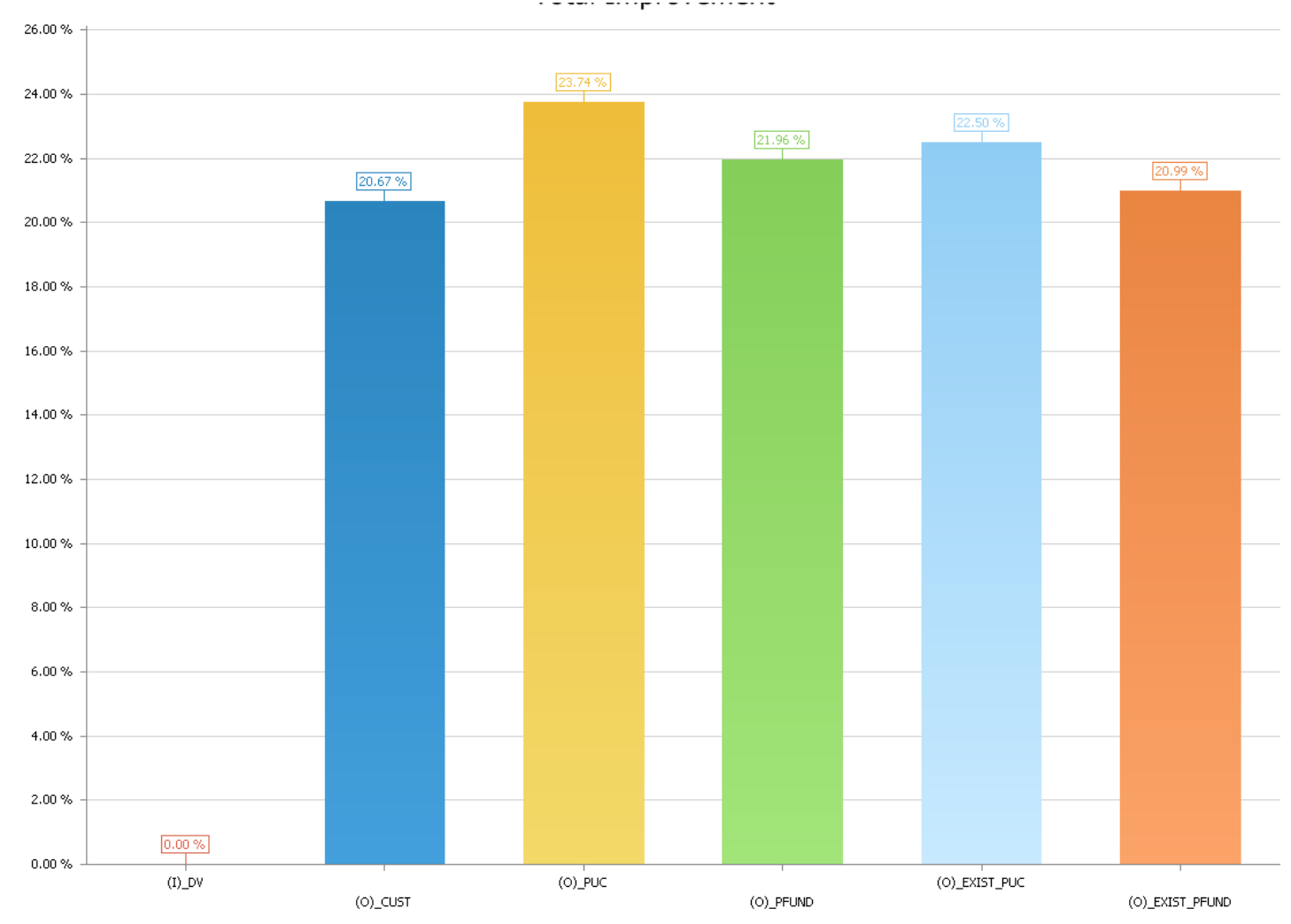




\section{A2.25- Run3}

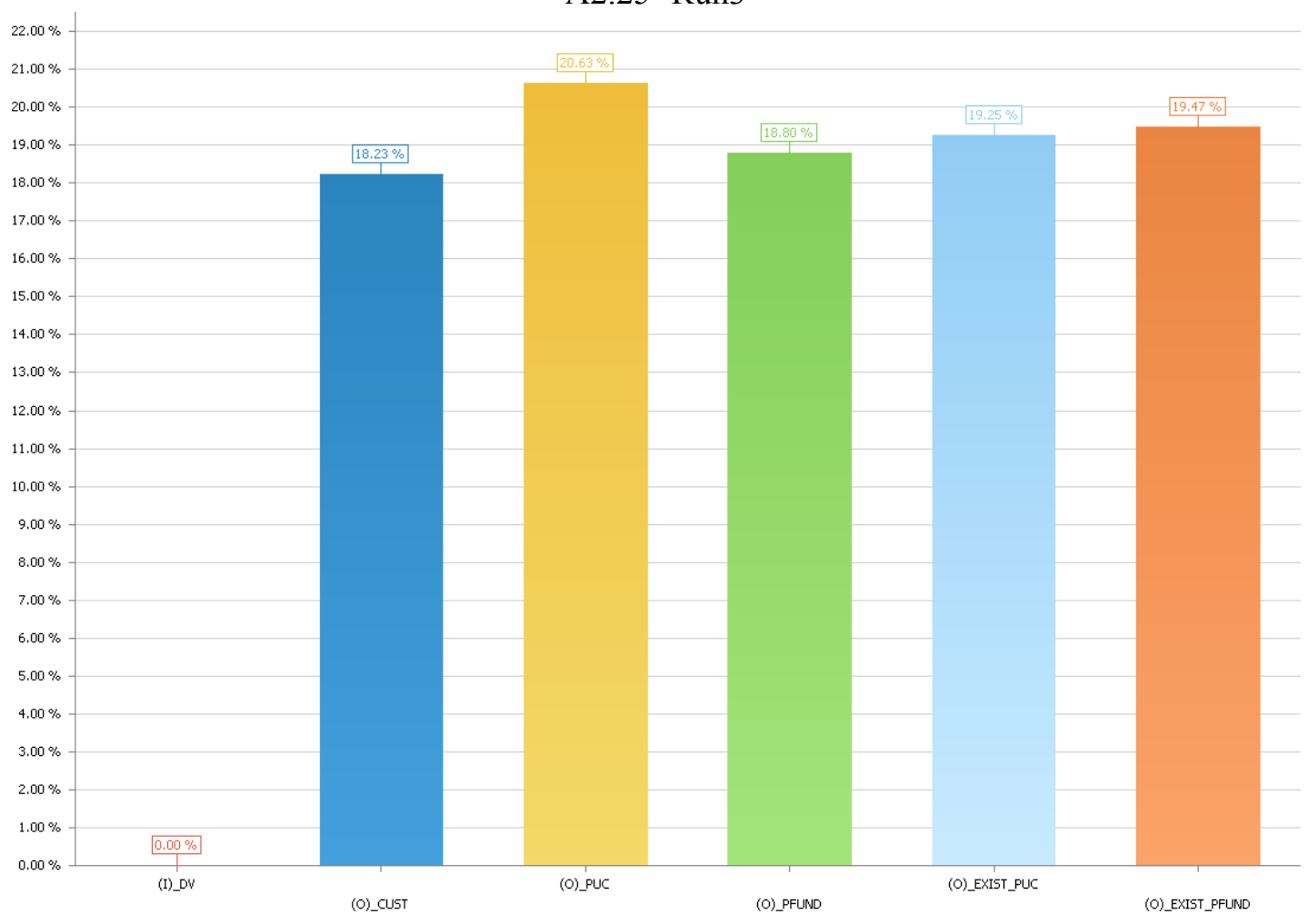

BCC Model:

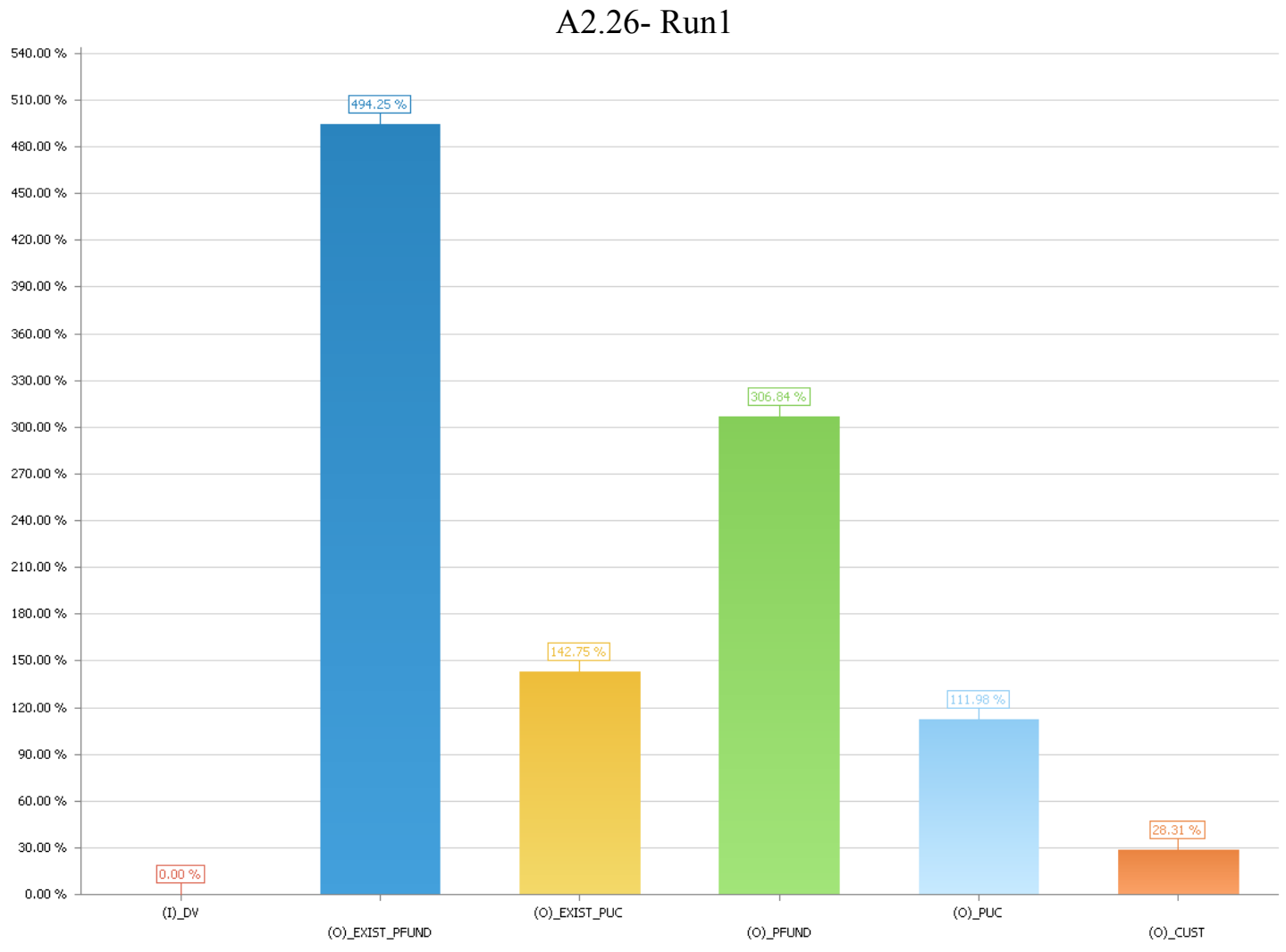


A2.27- Run 2

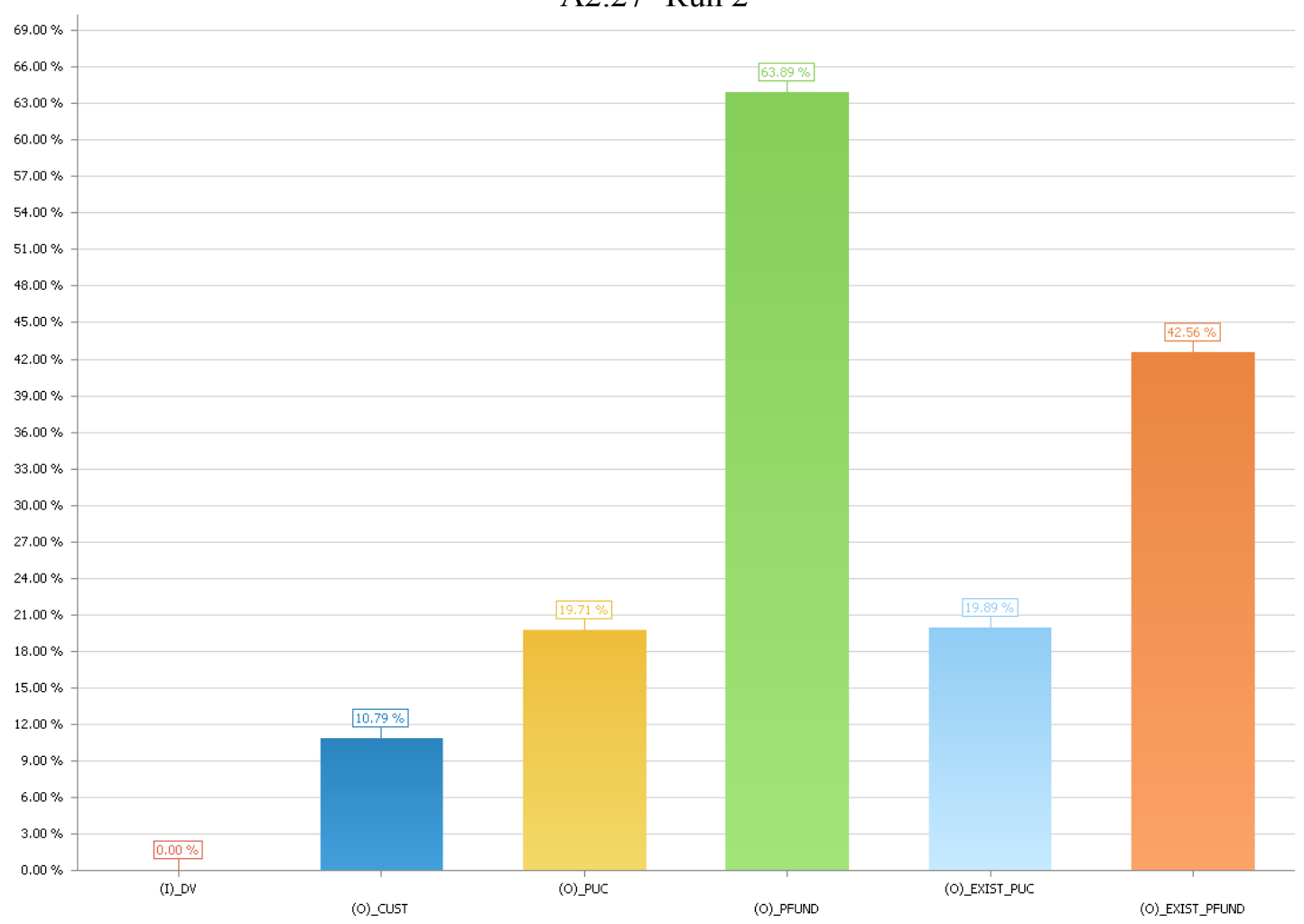

A2.28- Run 3

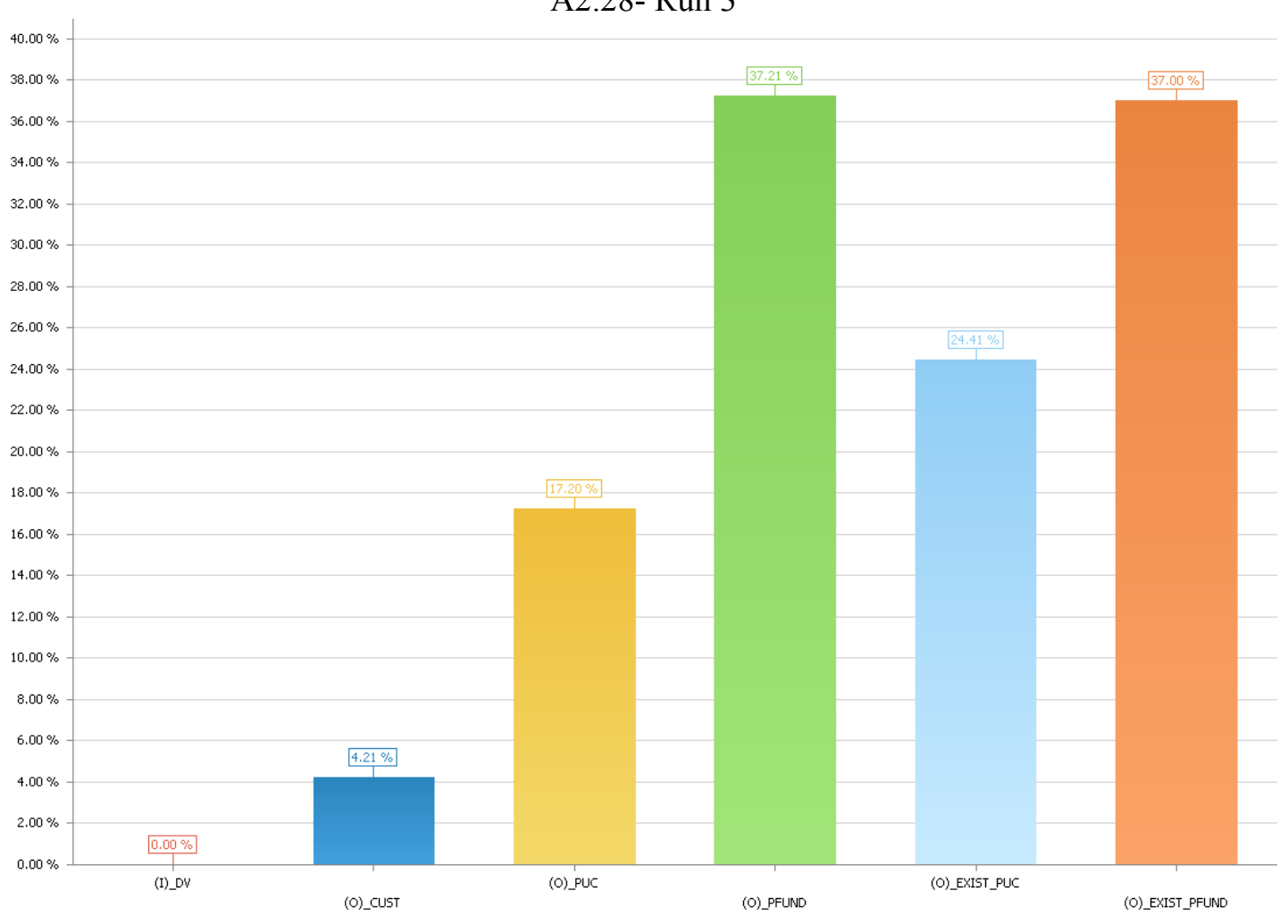




\section{Percent Model: Stripping Efficient Frontier}

CCR Model:

A2.29- Run1

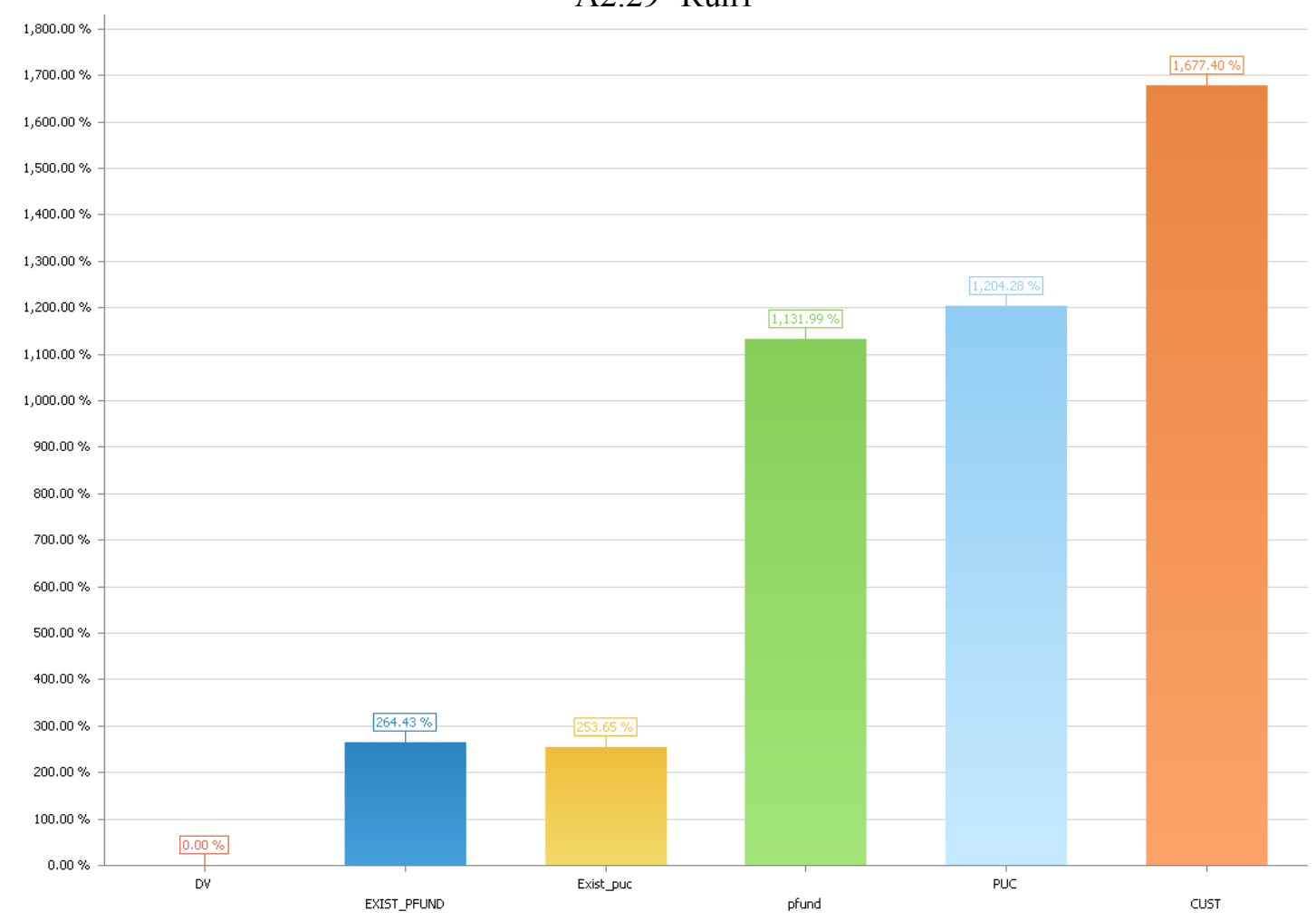

A2.30- Run2

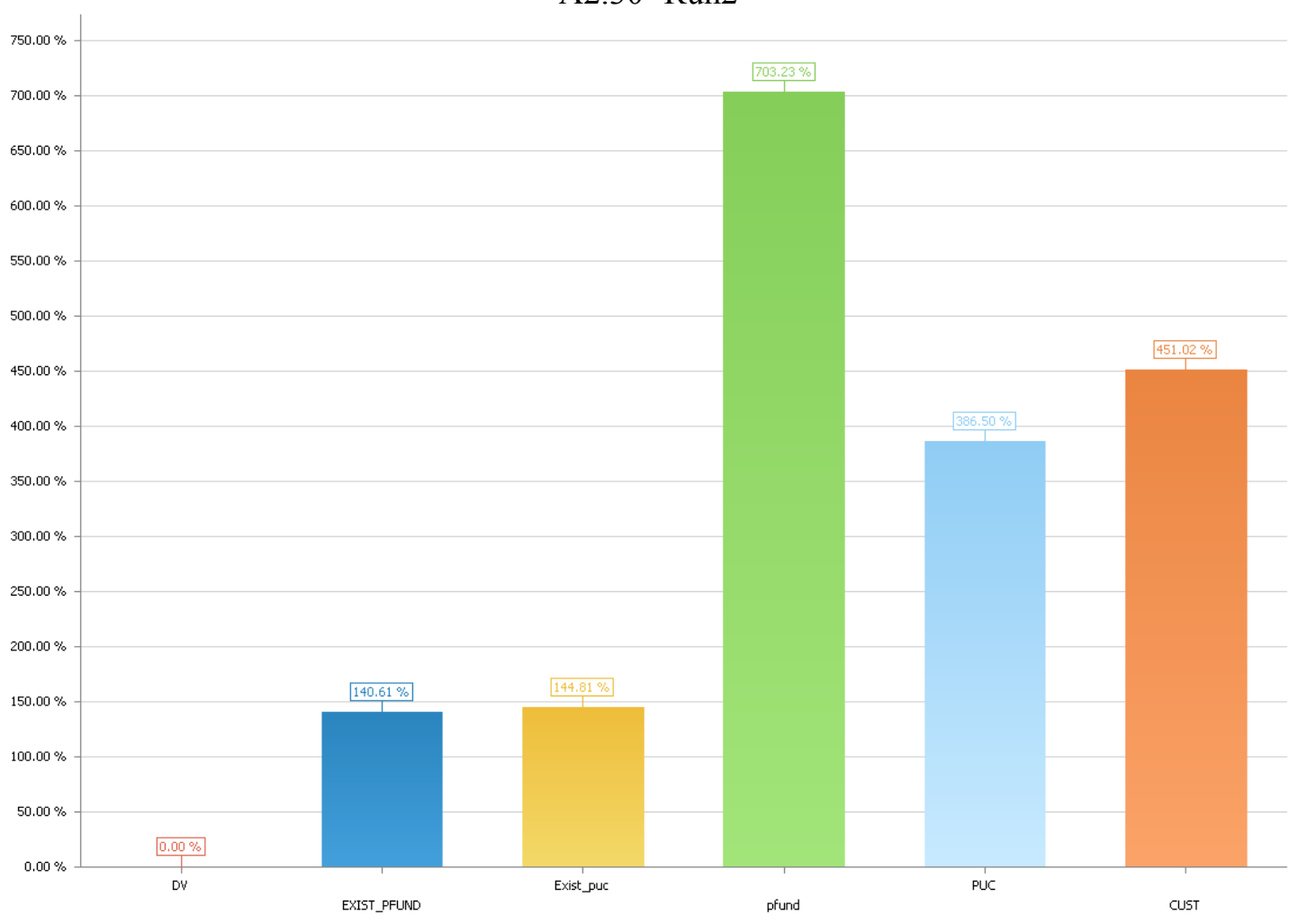




\section{A2.31- Run3}

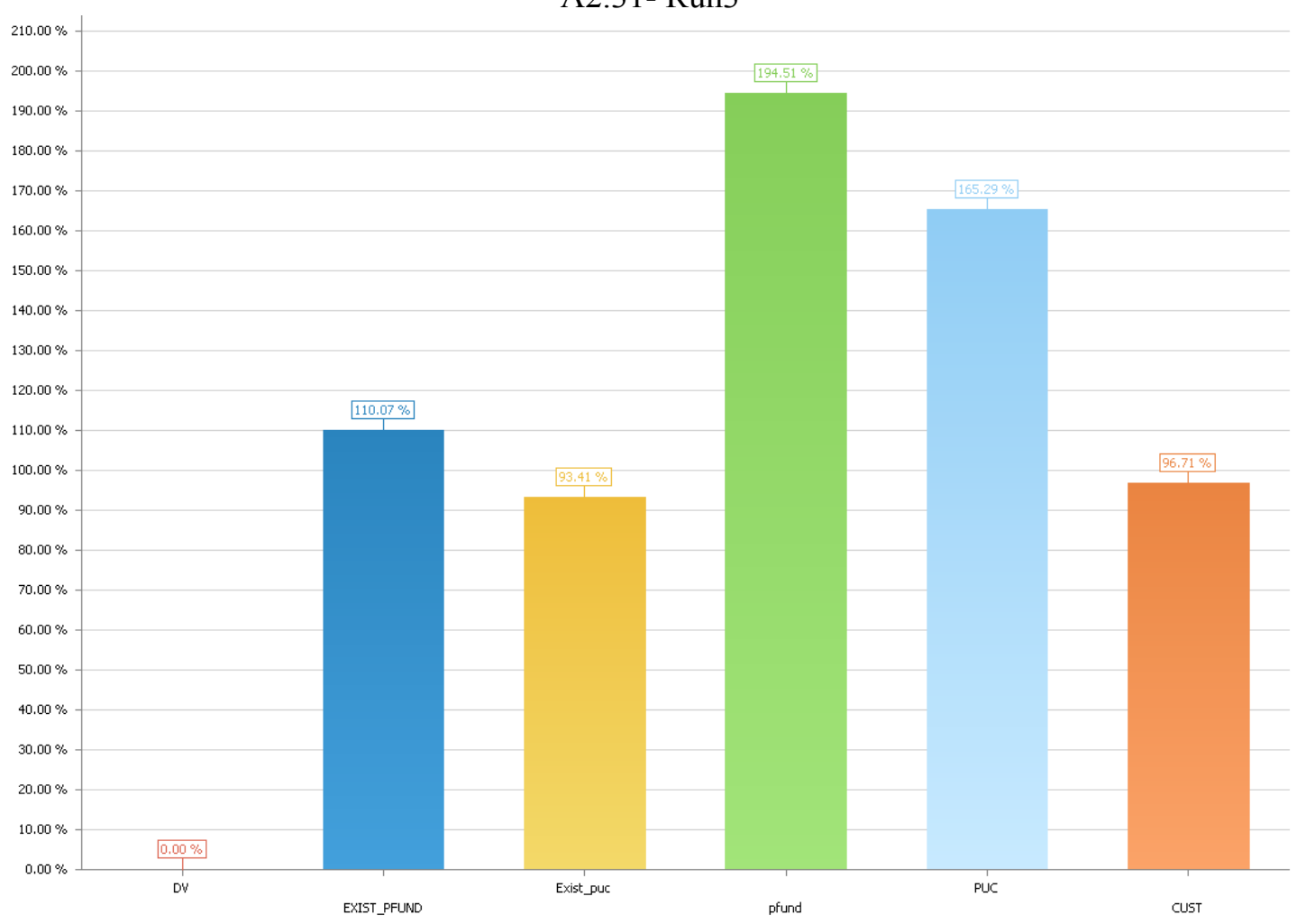

A2.32- Run4

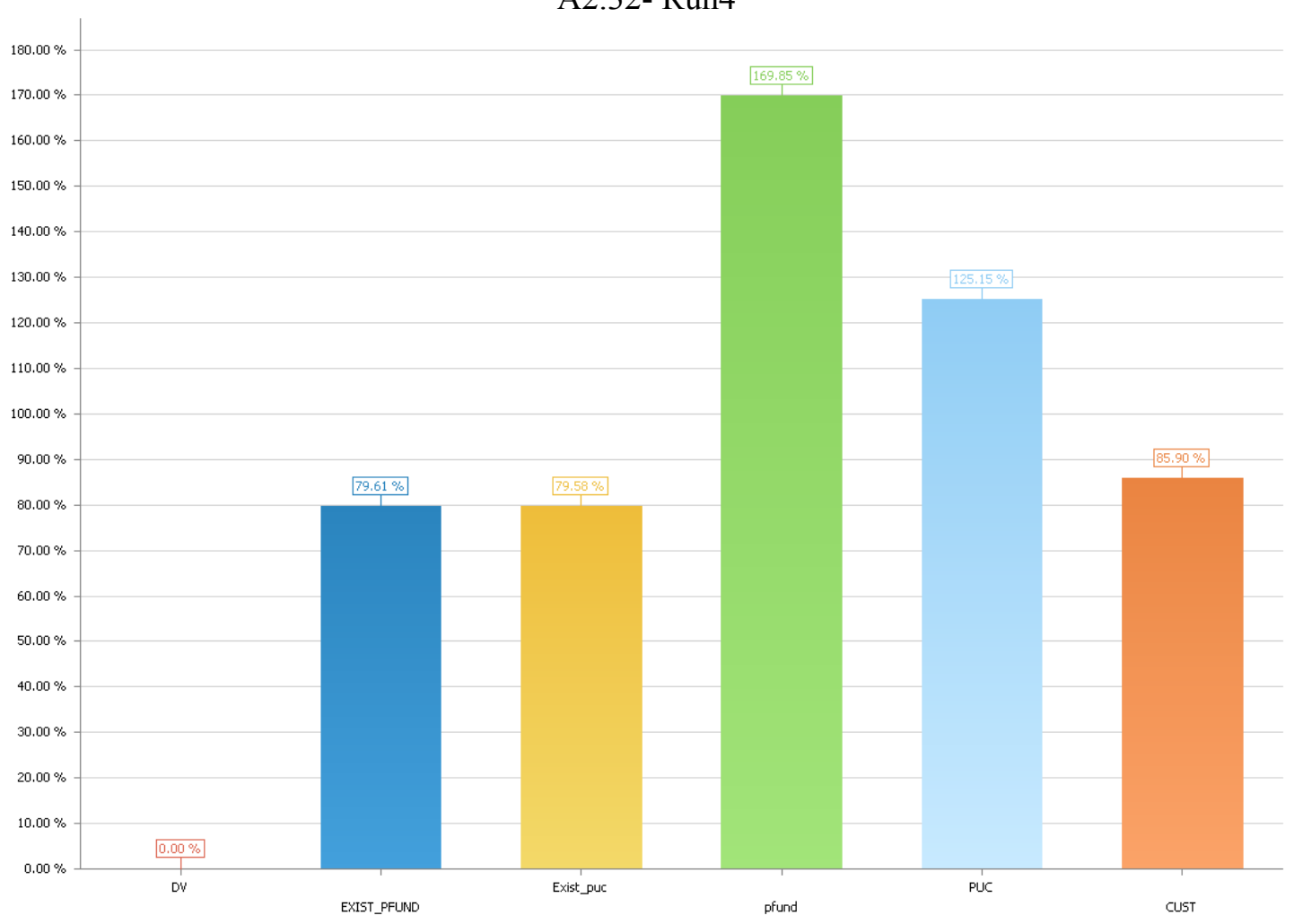




\section{SBM Model:}

A2.33- Run 1

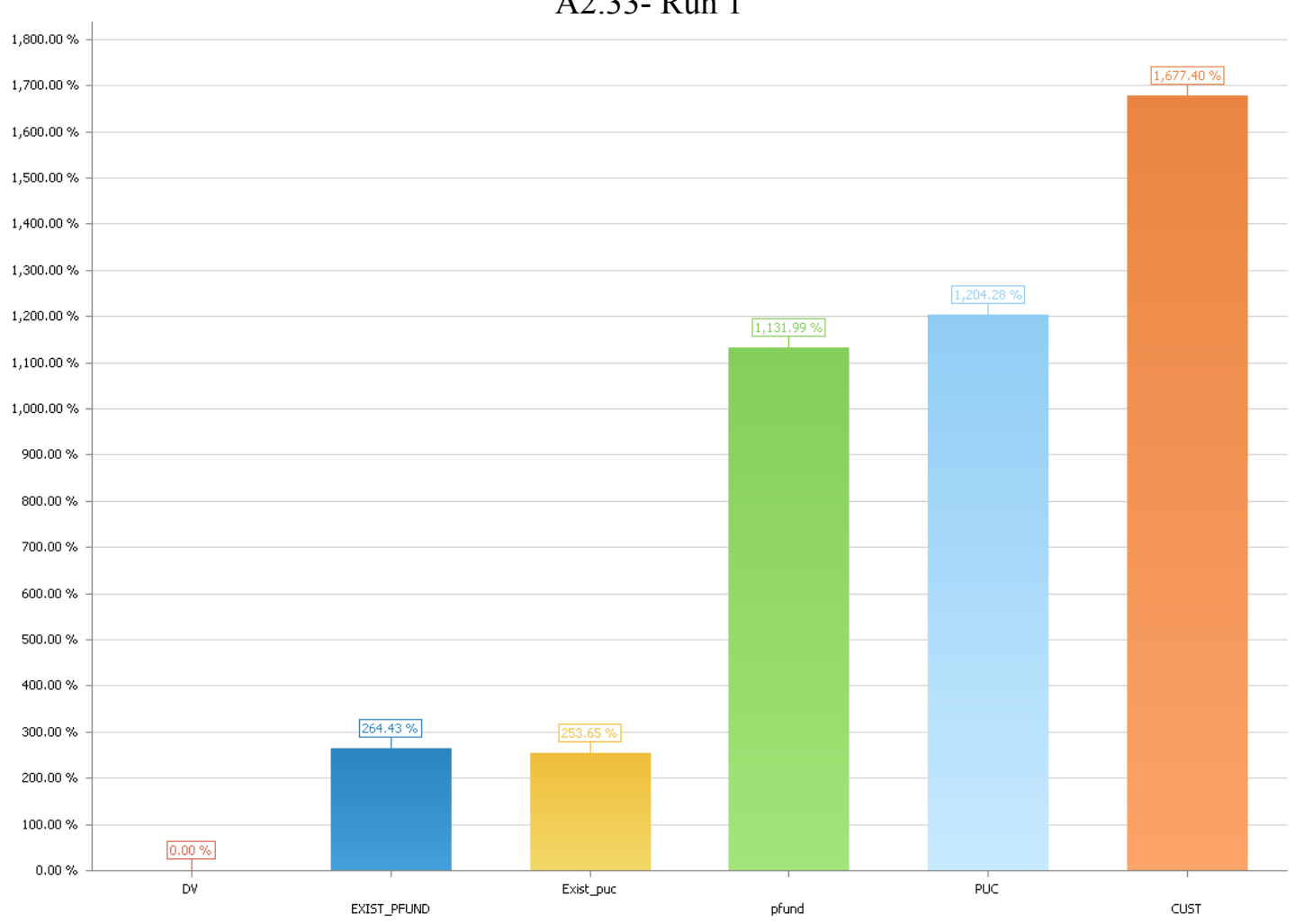

A2.34- Run 2

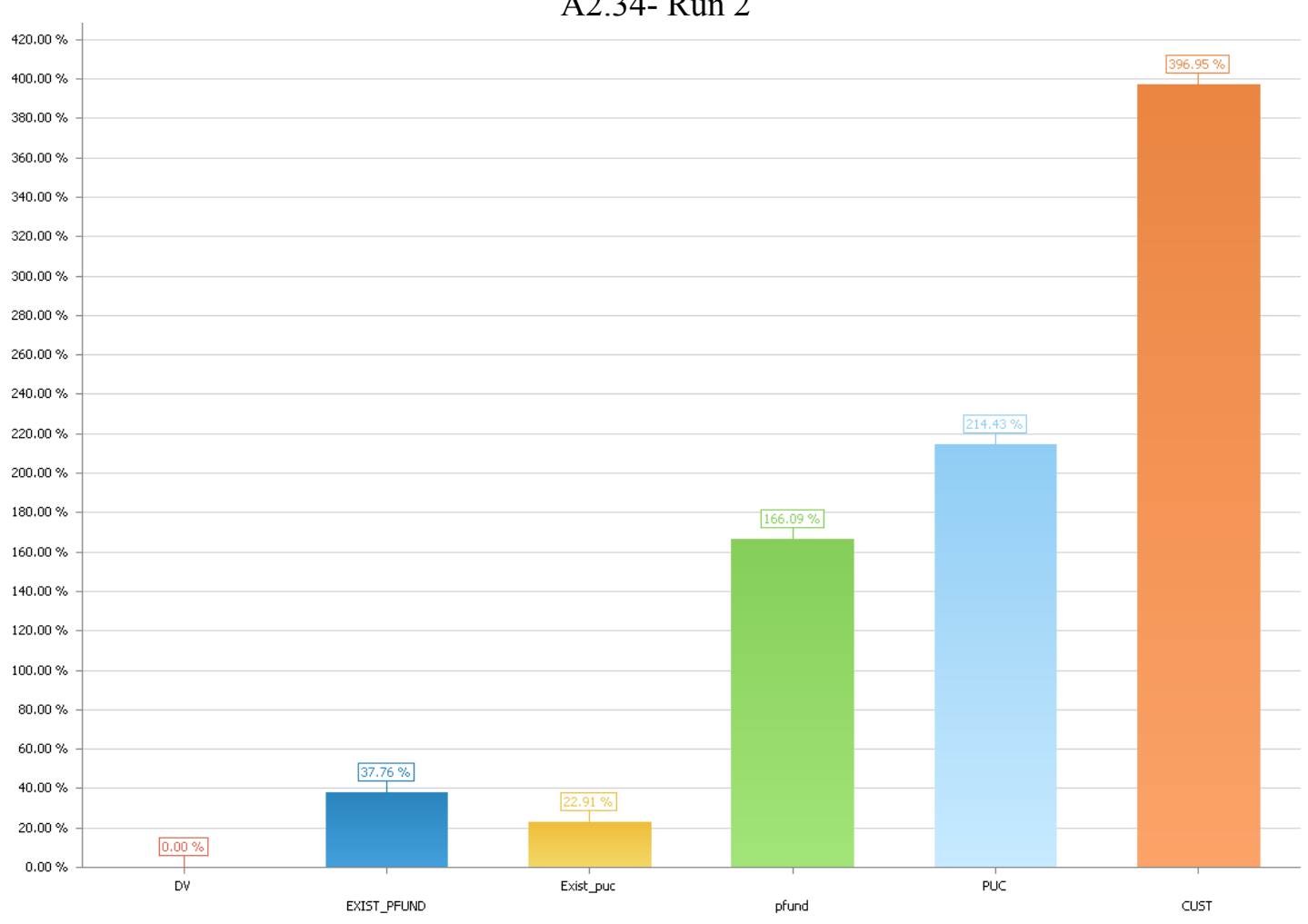




\section{Percent Model: Statistical Analysis and Local DEA}

CCR Model:

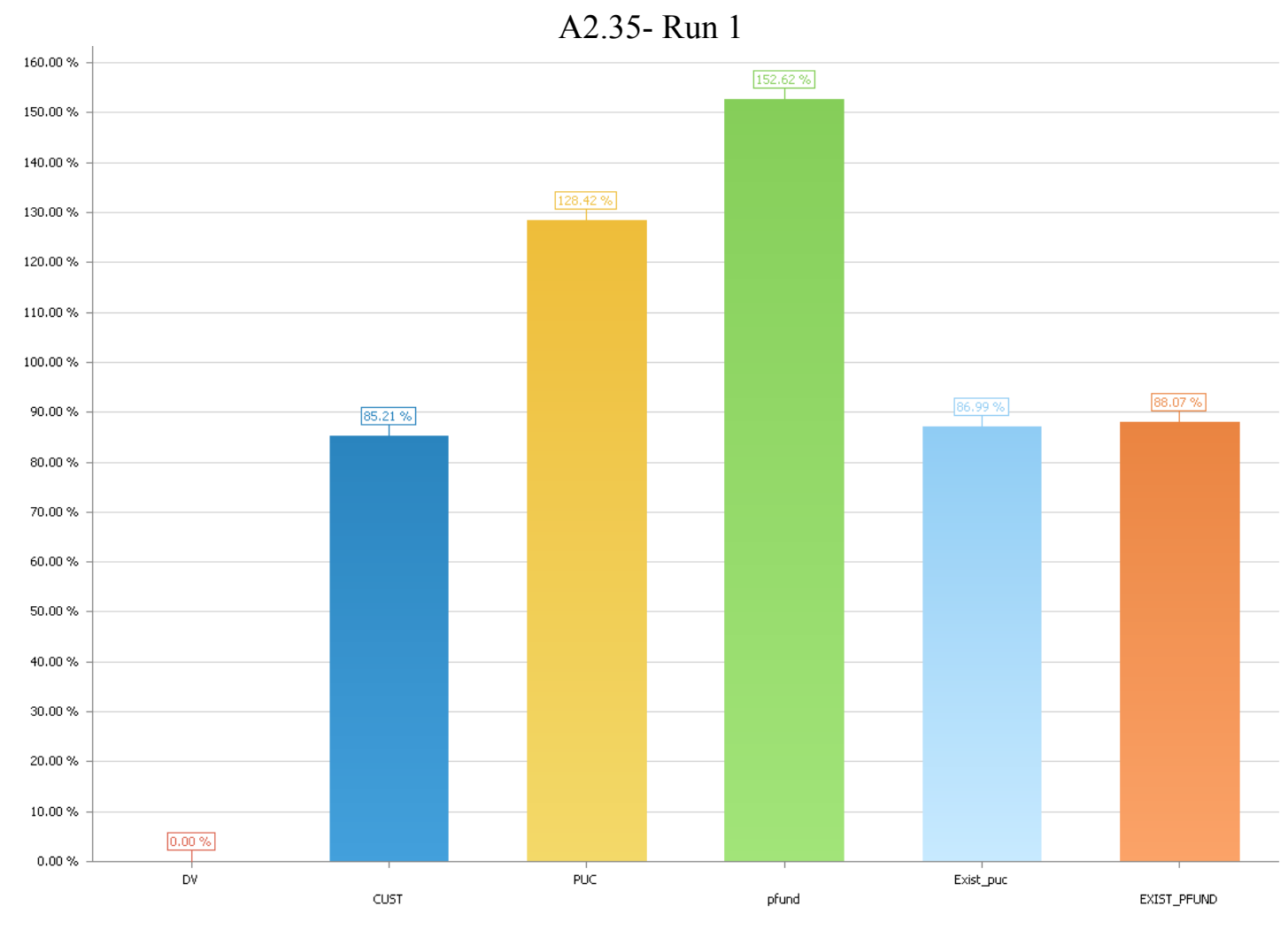

A2.36- Run 2

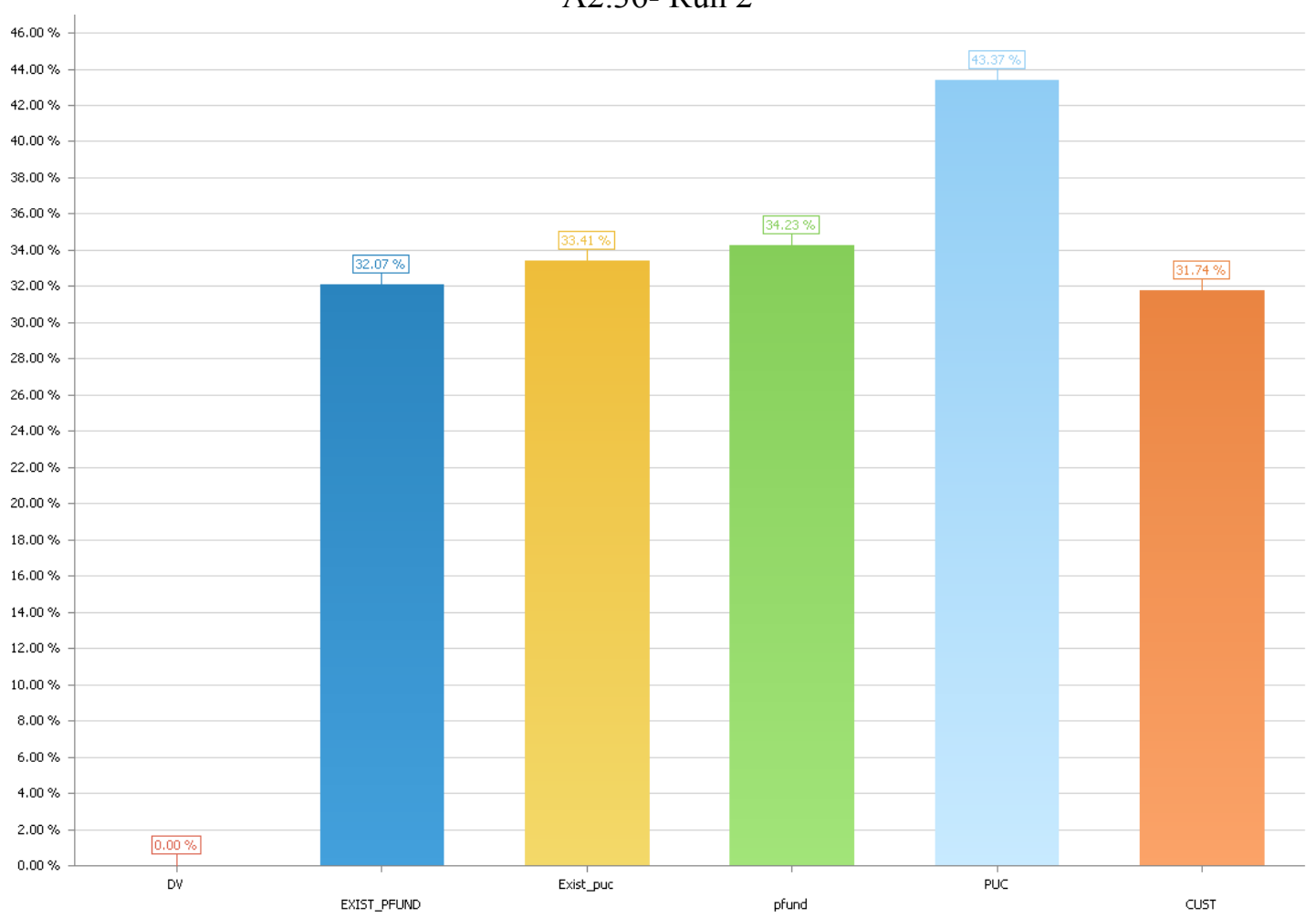


A2.37- Run 3

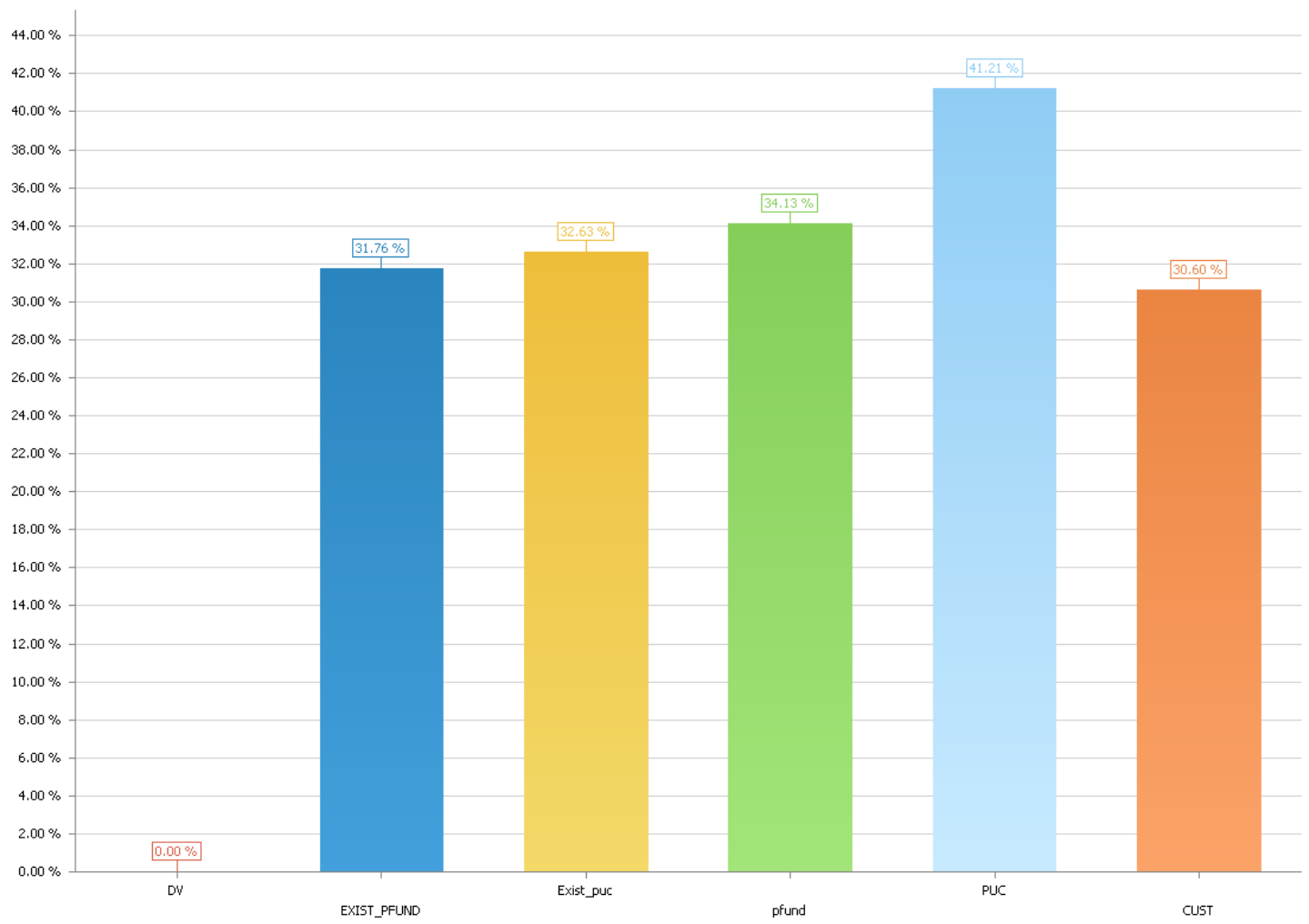

SBM Model:

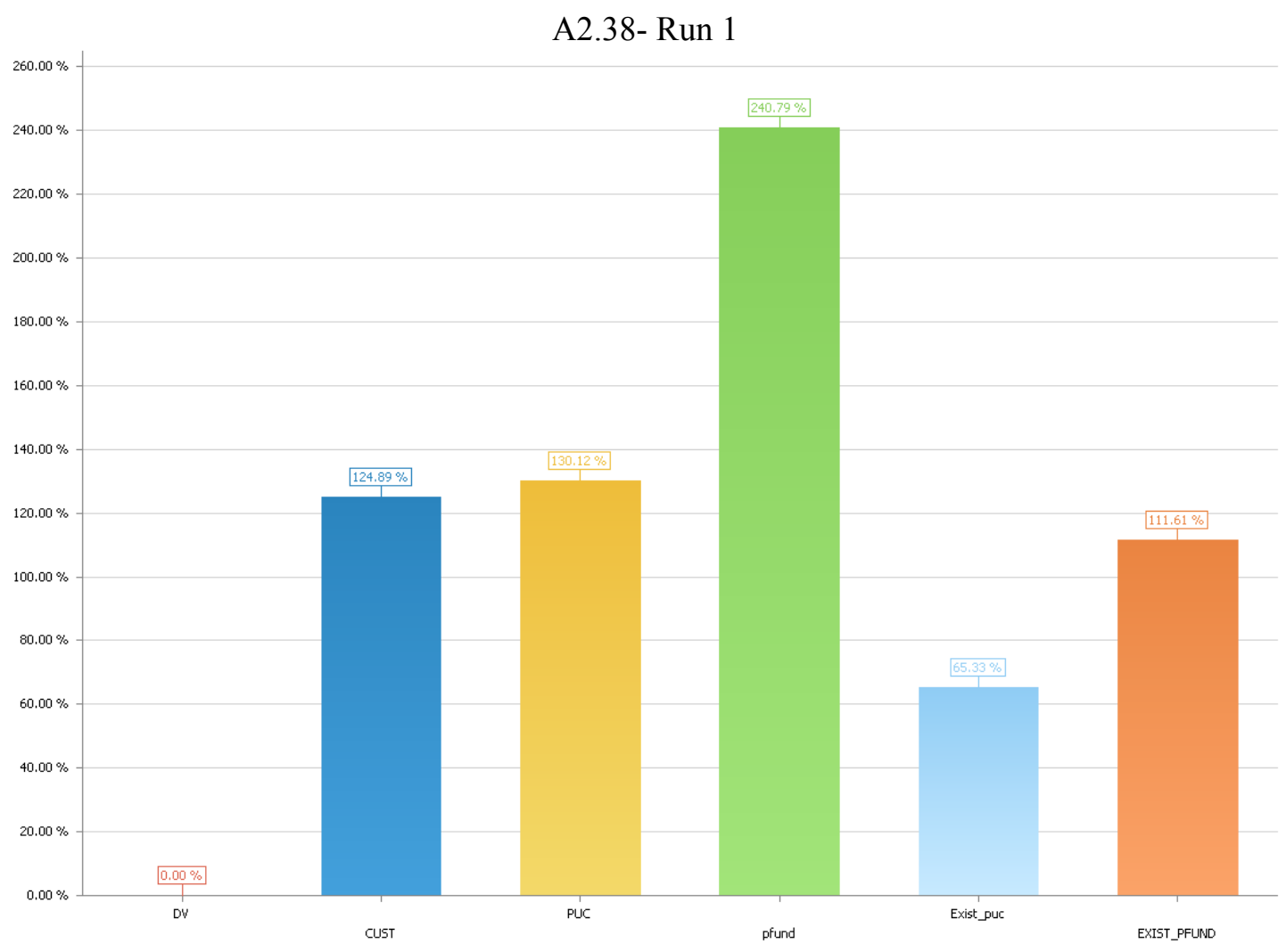


A2.39- Run 2

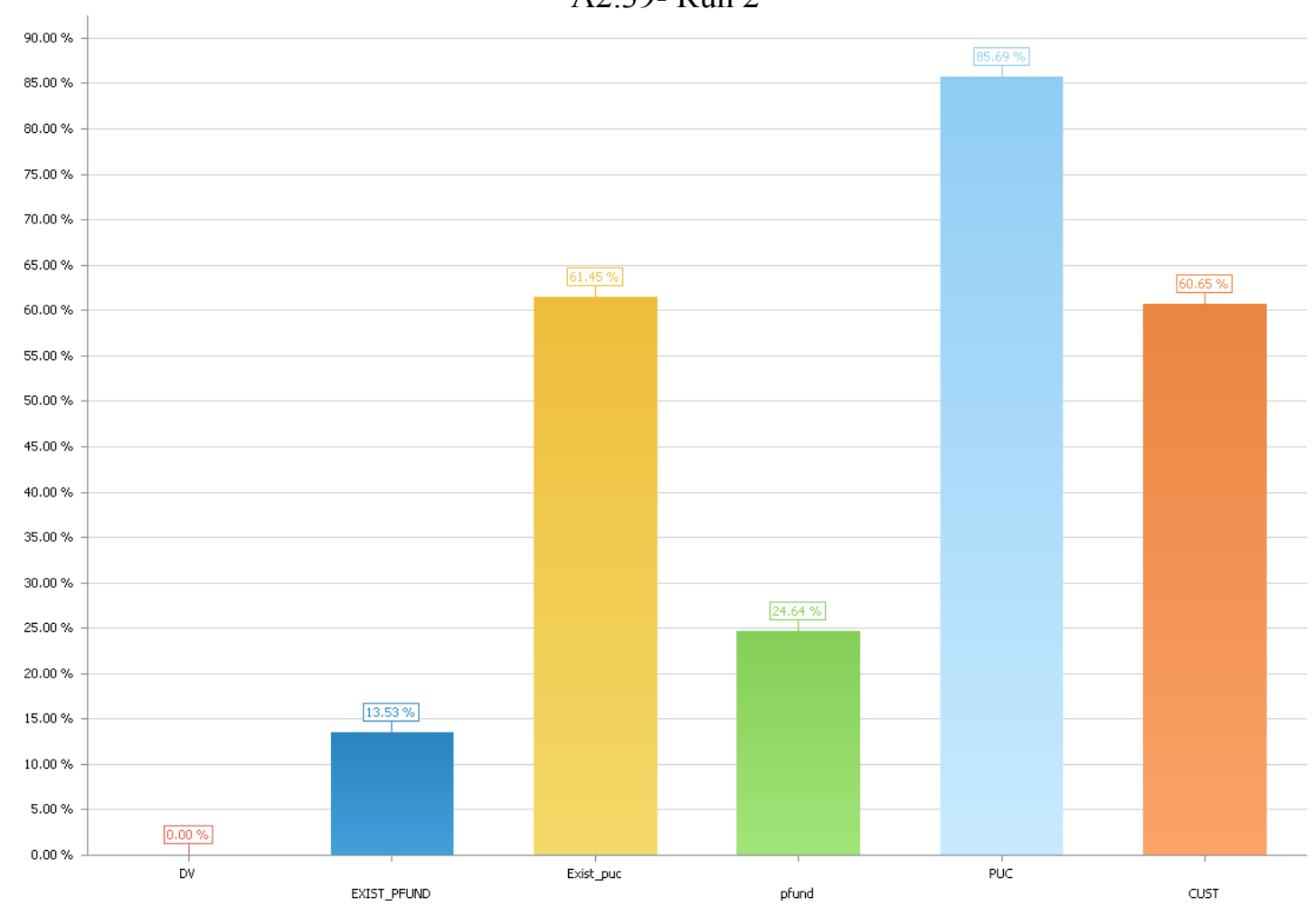

A2.40- Run 3

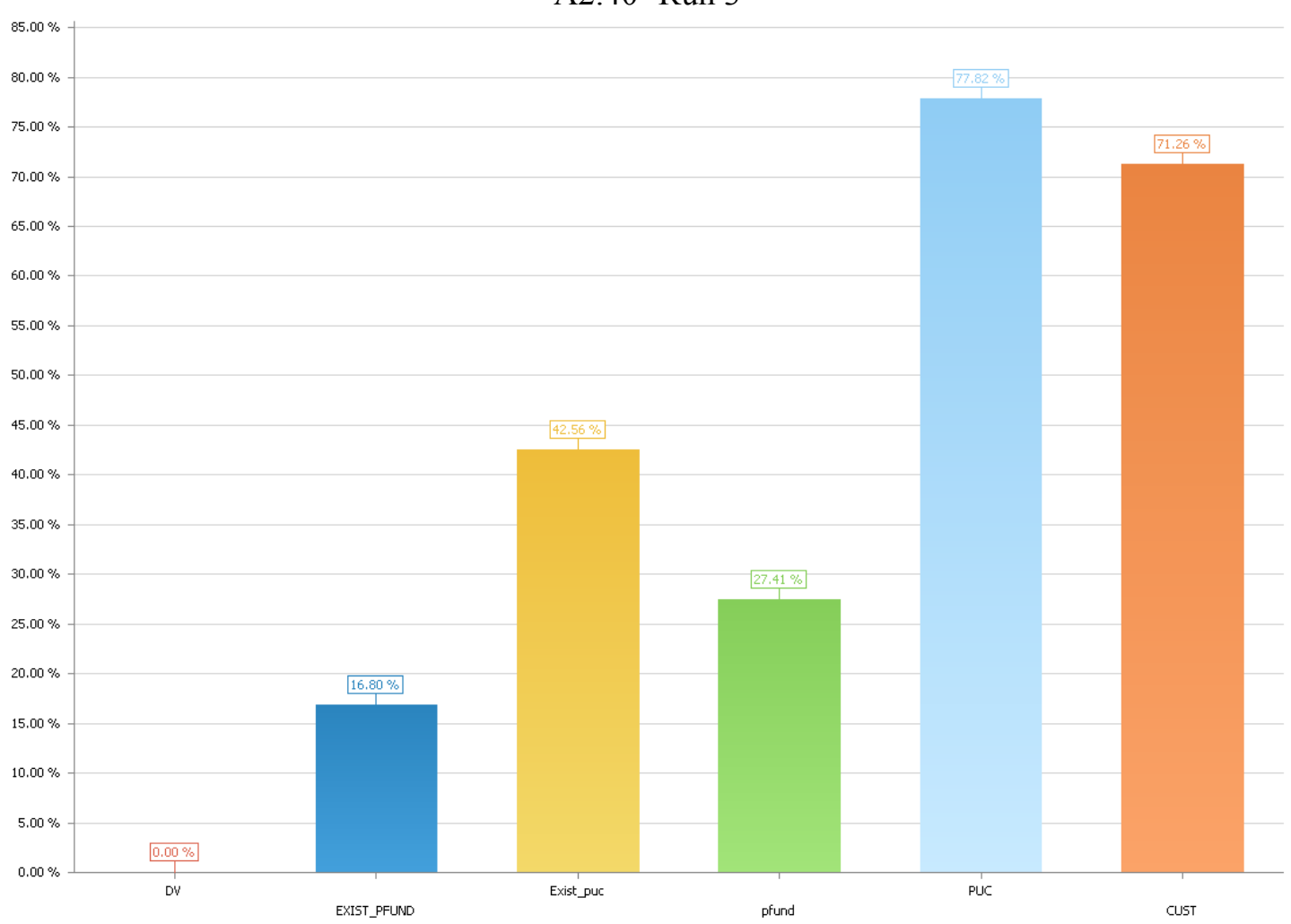

